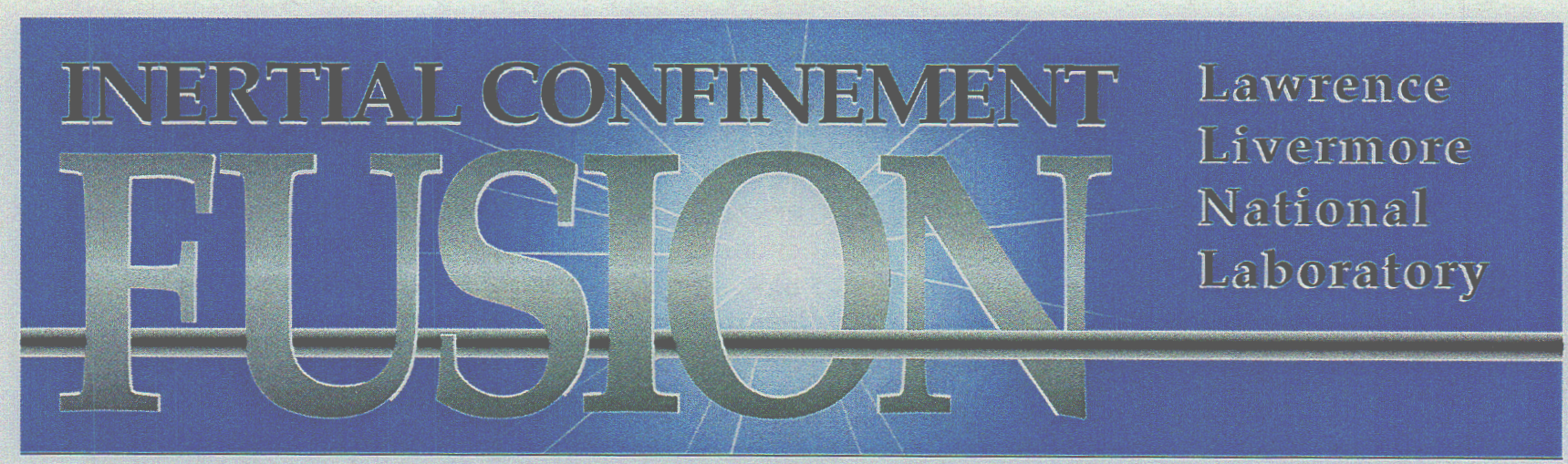

UCRL-LR-105820-99

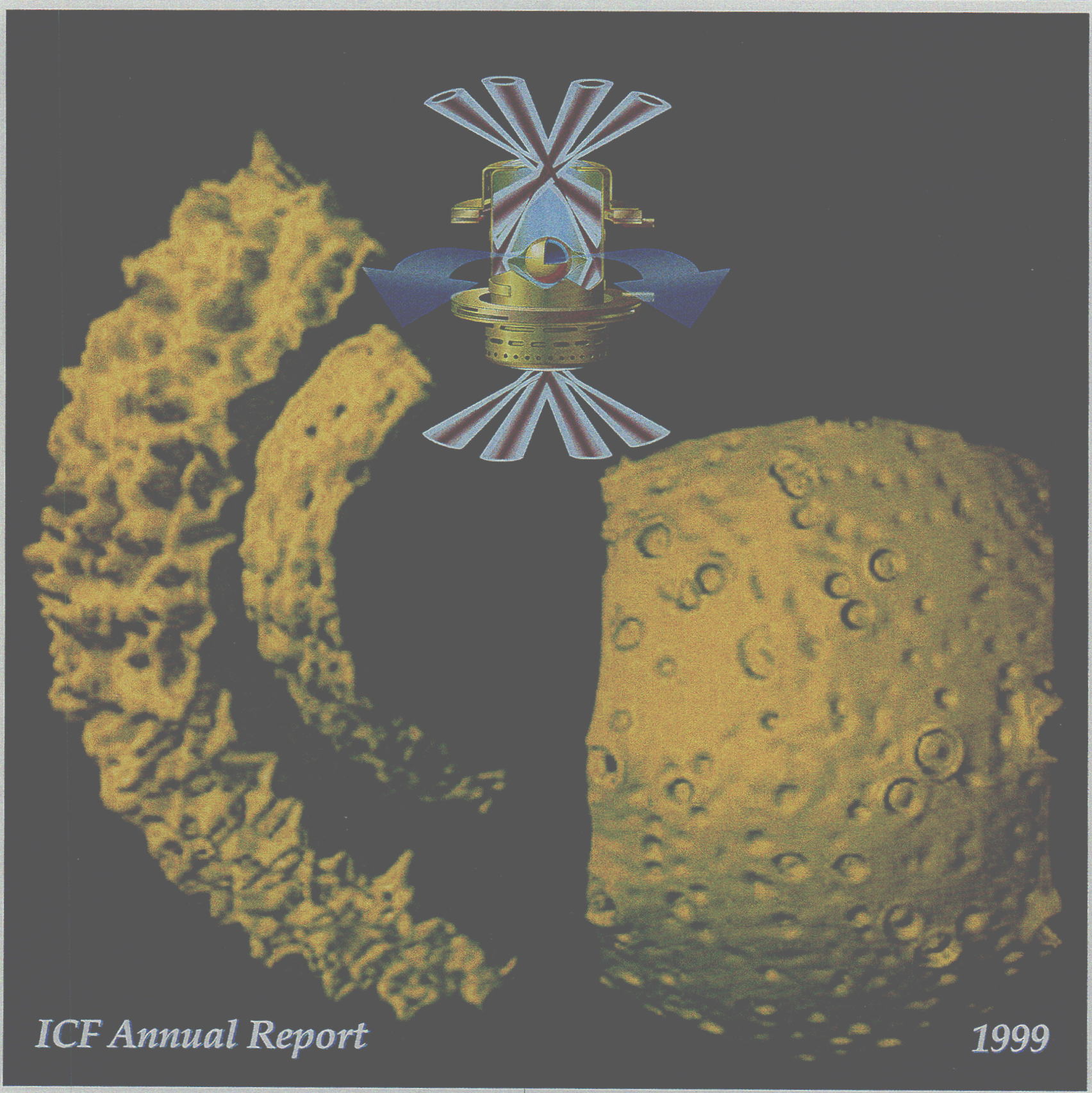


The Cover: The top-center figure is the gold cylinder containing the target capsule the size of a BB pellet. Laser beams with 1.8 million joules of energy (about 500 trillion watts) hit the cylinder. The cylinder then produces $x$-rays that create a rocket-like blowoff of the capsule surface, compressing and igniting the inner fuel core. Thermonuclear burn spreads rapidly through the compressed fuel, yielding many times the input energy. The left and right figures show results from a three-dimensional simulation of an ignition target. The plot on the left is a constant density surface of the implosion just prior to ignition showing nonlinear perturbation growth in the outer ablator (the larger sector of the sphere) and the imprint on the hot spot (the smaller sector). The figure on the right is a constant density plot of the rebounding shock at the time of ignition.

\section{On the Web:}

http://asers.llnl.gou/lasers/pubs/icfq.html

- UCRL-LR-105820-99

October 1998-September 1999

Printed in the United States of America

This document was prepared as an account of work sponsored by an agency of the United States Government. Neither the United States Government nor the University of California nor any of their employees makes any warranty, express or implied, or assumes any legal liability or responsibility for the accuracy, completeness, or usefulness of any information, apparatus, product, or process disclosed, or represents that its use would not infringe privately owned rights. Reference herein to any specific commercial product, process, or service by trade name, trademark, manufacturer, or otherwise, does not necessarily constitute or imply its endorsement, recommendation, or favoring by the United States Government or the University of California. The views and opinions of authors expressed herein do not necessarily state or reflect those of the United States Government or the University of California and shall not be used for advertising or product endorsement purposes.

\section{Available from
National Technical Information Service \\ U.S. Department of Commerce 5285 Port Royal Road \\ Springfield, Virginia 22161}

Price codes: printed copy A03, microfiche A01.

The work performed under the auspices of the U.S. Department of Energy by University of California Lawrence Livermore National Laboratory under Contract No. W-7405-Eng-48. 


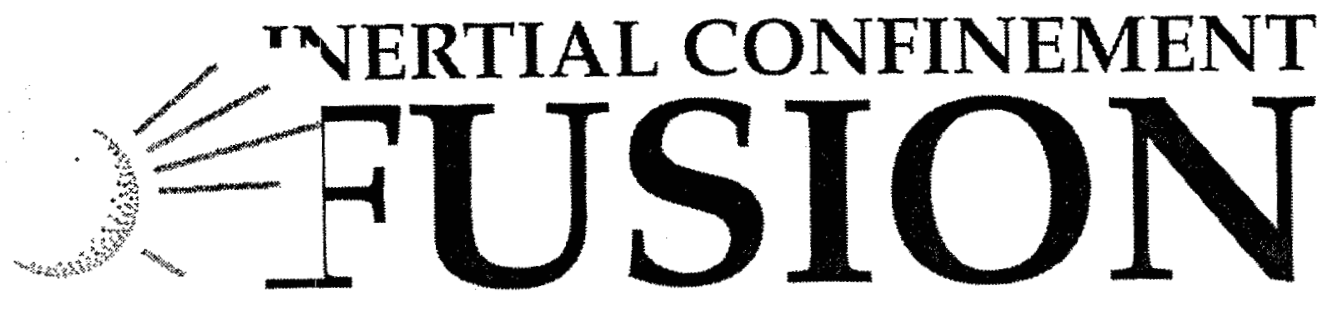

1999

ICF

Annual

Report

MS Date

July 2001

Lawrence Livermore

National Laboratory 



\title{
FOREWORD
}

The 1999 ICF Annual Report provides documentation of the achievements of the LLNL ICF/NIF and High-Energy-Density Experimental Science (HEDES) Program during the fiscal year by the use of two formats: (1) a summary of activity that is a narrative of important results for the fiscal year and (2) a compilation of the articles that previously appeared in the ICF Quarterly Report that year. In 1999, the compilation covers only three quarters and is being mailed separately in electronic format on a CD-ROM, which also includes this Annual Report. The Quarterly Report and this Annual Report are also on the Web at http://lasers.llnl.gov/lasers/pubs/icfq.html.

The underlying theme for LLNL's ICF/NIF Program research continues to be defined within DOE's Defense Programs Stockpile Stewardship missions and goals. In support of these missions and goals, the ICF/NIF and HEDES Program advances scientific and technology development in major interrelated areas that include laser target theory and design (much of it in support of the stockpile program), target fabrication, target experiments, and laser and optical science and technology. The ICF program provides the experimental support for much of the core weapons program work on high-energy lasers in support of stockpile stewardship.

While in pursuit of its goal of demonstrating thermonuclear fusion ignition and energy gain in the laboratory, the ICF/NIF and HEDES Program provides research and development opportunities in fundamental high-energy-density physics and supports the necessary research base for the possible long-term application of inertial fusion energy for civilian power production. ICF technologies continue to have spin-off applications for additional government and industrial use. In addition to these topics, the ICF Annual Report covers non-ICF funded, but related, laser research and development and associated applications. We also provide a short summary of the quarterly activities within Nova laser operations and in the construction of the National Ignition Facility.

The LLNL ICF/NIF Program is one, albeit the largest, part of the National ICF Program. The program is also executed at Los Alamos National Laboratory, Sandia National Laboratories, the University of Rochester, and the Naval Research Laboratory. General Atomics, Inc., develops and provides many of the targets for the above experimental facilities.

Questions and comments relating to the technical content of the journal should be addressed to the ICF Program Office, Lawrence Livermore National Laboratory, P.O. Box 808, Livermore, CA 94551.

\author{
Al Miguel/Jason Carpenter \\ Publication Editors \\ Joseph Kilkenny \\ ICF/NIF and HEDES Program Leader
}




\section{ACKNOWLEDGMENTS}

We thank the many authors and their co-authors (Bruce Hammel, Steve Haan, Gilbert Collins, Otto Landen, Peter Young, Warren Hsing, Christina Back, Kimberly Budil, Daniel Kalantar, Tom Bernat, Mary Spaeth, Bruno Van Wonterghem, Irv Stowers, Jack Campbell, Jerry Britten, Jeff Atherton, Tom Parham, Chris Stolz, Pam Whitman, Ruth HawleyFedder, Alan Burnham, and Trish Baisden) who contributed to this Annual Report. We are grateful for their willingness to take time from busy schedules to write the articles that describe their work. We thank Roy Johnson for his careful classification reviews. We also thank the administrative staff for typing manuscripts, arranging meetings, and offering other invaluable assistance.

We thank Technical Information Department (TID) editors Al Miguel and Cindy Cassady for editing and managing the production cycle; designer Pamela Davis for laying out this extensive document, Clayton Dahlen for designing the cover; and artists Dennis Chan, Al Garruto, Amy Henke, Linda Moore, Judy Rice, and Frank Uhlig for providing expertise in graphic design.

We appreciate the support of Michael Gallardo, the Government Printing Office coordinator, who worked with the Government Printing Office to obtain high-quality printing; and Mary Nijhuis of TID's Publications Services and TID's Print Plant for making sure that each publication was distributed with dispatch.

The talents and dedication of the ICF/NIF Program staff make the ICF Annual what it is for so many of its readers.

Robert L. Kauffman

Assistant DeputyAssociate Director

for ICF

Joseph Kilkenny

ICF/NIF and HEDES Program Leader 


\section{CONTENTS}

FOREWORD III

ACKNOWLEDGMENTS

INTRODUCTION

1.0 IgNition TARget Physics Experiments TheORY AND MODELING

2.0 High ENERGy Density EXPERIMENTAL SCIENCE 35

3.0 Target Development, Fabrication, AND Handling 45

4.0 NIF LASER DEVELOPMENT 61

$\begin{array}{ll}5.0 \text { Optics Technology DeVElopment } & 77\end{array}$

Appendix A: 1999 Awards, Patents, AND Refereed Publications $\quad$ A-1

ApPendix B: 1999 ICF QuARTERly Report ARTICles IN SEQUence B-1

Appendix C: Publications and Presentations $\quad$ C-1 



\section{INTRODUCTION}

$\mathrm{T}$ he ICF Program has undergone a significant change in 1999 with the decommissioning of the Nova laser and the transfer of much of the experimental program to the OMEGA laser at the University of Rochester. The Nova laser ended operations with the final experiment conducted on May 27, 1999. This marked the end to one of DOE's most successful experimental facilities.

Since its commissioning in 1985, Nova performed 13,424 experiments supporting ICF, Defense Sciences, high-power laser research, and basic science research. At the time of its commissioning, Nova was the world's most powerful laser. Its early experiments demonstrated that $3 \omega$ light could produce high-drive, low-preheat environment required for indirect-drive ICF.

In the early 1990s, the technical program on Nova for indirect drive ignition was defined by the Nova technical contract established by National Academy Review of ICF in 1990.* Successful completion of this research program contributed significantly to the recommendation by the ICF Advisory Committee in 1995 to proceed with the construction of the National Ignition Facility. ${ }^{\dagger}$ Nova experi-

*Review of the Department of Energy's Inertial Confinement Fusion Program, National Academy of Sciences, September, 1990.

${ }^{\dagger}$ Inertial Confinement Fusion Advisory Committee Letter Report 4, February 21, 1996. ments also demonstrated the utility of high-powered lasers for studying the physics of interest to Defense Sciences. Now, high-powered lasers along with pulsed-power machines are the principal facilities for studying high energy density science in DOE's Stockpile Stewardship Program (SSP).

In 1997, one beam of Nova was converted to a short pulsed beam producing a petawatt of power in subpicosecond pulses. The petawatt beam was used for pioneering research in short-pulse laser-matter interactions relevant to fast ignitor ICF and short pulsed $x$-ray, electron, and particle production for use as probes.

Nova is being disassembled and the space is being used to support NIF construction. Nova components are being distributed to a number of other laser laboratories around the world for reuse as determined by DOE.

This report summarizes the research performed by the ICF Program in FY1999. The report is divided into five sections corresponding to the major areas of program activities. These are sections on (1) ignition target physics experiments theory and modeling, (2) high energy density experimental science, (3) target development, fabrication, and handling, (4) NIF laser development, and (5) optics technology development.

The ignition target physics experiments, theory, and modeling effort continues its work on better understanding the physics basis for the ignition target and on increasing the likelihood of reaching ignition on the NIF. Highlights for 1999 include: 
- Observation of the Langmuir decay instability using Thomson scattering on Nova.

- Measurement of the angular distribution of forward-scattered light in NIF-like plasmas.

- First experiments in long-scalelength $\mathrm{He} / \mathrm{H}$ plasmas at densities planned for the NIF.

- Simulation of full-scale NIF beam propagating through an underdense plasma using pF3D.

- Measurement of angular distribution of $x$-ray radiation from a hohlraum on OMEGA.

- Start of developing techniques for measuring the radiation symmetry during the foot of a NIF pulse.

- Initial implosion experiments to study the performance of higher convergence $(\sim 20)$ targets.

- Calculation of NIF capsule sensitivity to different EOS and opacity models.

- Demonstration of the ability to measure shock velocities up to the third shock of an ignition capsule using VISAR.

- Completion of integrated calculations and analysis for a $350-\mathrm{eV}$ Be capsule ignition design.

- 3D simulations of a NIF capsule including low-mode RayleighTaylor effects.

- Installation of a second charged particle spectrometer on OMEGA in collaboration with scientists from Massachusetts Institute of Technology and the University of Rochester.

High Energy Density Experimental Science performed experiments on Nova and OMEGA in collaboration with the Defense and Nuclear Technologies Directorate, Defense Threat Reduction agency on x-ray sources, and the Physical Database Research Program on Equation of State (EOS). Highlights for 1999 include:

- Hydrodynamic instability experiments on Nova with $\mathrm{Al}$ in regimes where material strength is important.
- Planar direct-drive experiments measuring diffraction of compressed $\mathrm{Cu}$ from two orthogonal lattice planes.

- Observation of hydrodynamic instability growth in doubly shocked experiments.

- Measurement of diffusive radiation flow in OMEGA experiments.

- Off-Hugoniot equation-of-state measurements in deuterium.

The Target Development, Fabrication, and Handling efforts are focused on developing the technology to manufacture and field an ignition target on the NIF. Some 1999 highlights are:

- Demonstration of smoother sputtered Be capsules using a new coating geometry.

- Manufacture of polyimide capsules with wall thickness greater than $100 \mu \mathrm{m}$.

- Production of NIF capsule mandrels close to required specifications for roughness and roundness.

- Demonstration of smooth DT layering at $0.5^{\circ} \mathrm{K}$ below triple point.

- Activation of the cryogenic Hohlraum Test Facility.

Highlights for 1999 in NIF laser development are:

- Correlation of damage spot size on amplifier slabs with contamination particle size.

- Development of processing procedures for clean assembly of amplifiers.

- Demonstration of key NIF performance requirements with the engineering prototype of the preamplifier module (PAM).

- Qualification of vendors for NIF capacitors and placement of initial procurements.

- Placement of contract for high bandwidth $3 \omega$ optical fiber for NIF.

- Completion of characterization tests of a prototype NIF deformable mirror. 
The Optics Technology Development effort develops the technology and capability for producing the specialized optics required for NIF. Highlights in 1999 include:

- Investigation of alternate crystal growth configurations to improve yield of nonlinear crystals.

- Decision to use wedged lens final focusing geometry obviating the use of color separation gratings.

- Demonstration of full-size stabilized sol-gel antireflection coatings on fused silica.
- Completion of initial pilot operation of continuous amplifier glass pours at Schott Glass Technologies Inc. and Hoya Corporation.

- Completion of facilitization of the NIF optics finishing facilities at Zygo Corporation.

- Beginning of pilot operations at University of Rochester Laboratory for Laser Energetics (LLE) and Spectra-Physics on mirror and polarizer coatings and at Cleveland Crystals, Inc., on rapid growth KDP. 



\subsection{Ignition TARget Physics EXPeriments THEORY AND MODELING}

$\mathrm{D}$ uring the past year our program of work has continued to follow the high-priority activities defined in the National Ignition Plan. This plan breaks the activities that are required to reach ignition into the following four subject areas:

1. Hohlraum Energetics.

2. Hohlraum Symmetry.

3. Implosion Optimization and Shock Timing.

4. Implosion Physics-Target Design, Advanced Diagnostics Development, and Target Fabrication.

There have been significant advances this year in all four areas of the Ignition work breakdown structure (WBS). In the area of Hohlraum Energetics, we performed a successful series of experiments, using cryogenic gasbag targets, examining ion wave damping of laser plasma instabilities (LPI). We also used Thomson Scattering to study saturation mechanisms of these instabilities. In the area of Hohlraum Symmetry, we have continued to develop techniques for symmetry measurement and control that are required for ignition on the National Ignition Facility (NIF). Using the NIF-like illumination capabilities on the OMEGA laser at the University of Rochester, we have continued our implosion experiments at higher convergence ratio than previously possible. In the area of Implosion Optimization and Shock Timing, we successfully used a proposed shock-timing method for NIF ignition capsules, to observe a strong shock overtake a weaker one in liquid deuterium. Finally, there has been excellent progress in the area of Implosion Physics. Target design has continued to focus on increasing our understanding of the requirements for the baseline targets (point design) and on developing target designs that work on the edges of ignition-parameter space. Progress on target fabrication has occurred on all fronts, with advances in capsule development and cryogenic layering and control. (For more details about our target fabrication work this year, see Section 3.0, "Target Development, Fabrication, and Handling" on p. 43.)

\section{Hohlraum Energetics}

In fiscal year 1999 (FY99), we continued to make progress in advancing our experimental and theoretical understanding of the laser-plasma instability (LPI) target physics required to optimize the performance of the NIF ignition targets. Efficient conversion of laser light to $x$-rays at the hohlraum walls of indirect-drive ignition targets is important for high-gain implosions. The conversion efficiency can be reduced by instabilities such as stimulated Brillouin scattering (SBS) and stimulated Raman scattering (SRS) that can potentially scatter incident laser light before it can couple into the ignition hohlraum. Furthermore, SRS can produce hot electrons that can preheat the ignition capsule. Control of LPI using beam smoothing techniques such as smoothing by spectral dispersion (SSD) has been demonstrated in 
Nova experiments, but work continues to understand the behavior of LPI with plasmas of larger size and higher electron temperature expected in NIF targets.

Analysis of Nova experiments and further development of the LPI physics code pF3D have tested physics related to beam propagation in underdense plasmas and studied saturation mechanisms SBS and SRS. Of particular importance was the fullscale $\mathrm{pF3D}$ simulation of Nova-sized gasbag and hohlraum targets (see subsection "Full-Size pF3D Simulation of Gasbag and Scale 1 NIF Plasmas"). The pF3D simulations were used to interpret Nova experiments that investigated the forward scattering of light propagating through an underdense gasbag plasma.

Another important result was the fielding of cryogenic gasbags to test the effect of ion wave damping on the levels of SBS and SRS. The damping, which covers the range expected in NIF ignition targets, was controlled by varying the gases in the gasbag (see subsection "LPI Experiments with $\mathrm{He} / \mathrm{H}$ Gas Mixture on Nova"). SRS was seen to be nearly independent of the damping while SBS decreased monotonically with increasing damping.

Further results reported in this issue include the observed saturation of SRS due to the Langmuir decay instability (LDI). The Nova experiments used Thomson scattering to identify the waves associated with LDI. We have also examined ways to change to phase plate design, which will allow us eventually to prescribe a NIF phase plate design that will minimize the levels of SBS and SRS.

\section{Nova Scaling Experiments of SRS, SBS, Forward Scattering, and Saturation}

Proposed targets for achieving ignition on the NIF laser will be larger and have higher temperature than in previous experiments. Prediction of NIF SBS and SRS $^{1}$ instability levels relies on understanding the dependence of instability growth on plasma parameters and the conditions under which the instabilities saturate. New tools have been developed, which have increased our confidence in predicting and controlling instabilities in
NIF plasma conditions. For example, Thomson scattering ${ }^{2}$ on Nova has led to detailed measurements of the plasma waves associated with SBS and SRS, and a parallel version of the nonlinear hydrodynamic code F3D can now model Novasized problems. ${ }^{3}$

Our studies are organized into two categories. First, a number of Nova experiments were conducted to identify the saturation mechanism for SRS and to measure the saturated fluctuation level of the SBS ion wave. In addition, cryogenic targets were used to examine the dependence of instability levels on ion wave damping. Second, beam smoothing techniques have been investigated. Possible NIF beam smoothing techniques include KPPs, polarization smoothing, ${ }^{4,5}$ and SSD. An important component of this study is the development of a parallel form of F3D$\mathrm{pF} 3 \mathrm{D}$, which is capable of modeling millimeter-sized plasmas, therefore making it easier to take into account plasma effects on the laser propagation through the target plasma.

\section{SBS and SRS Saturation Experiments}

The Langmuir decay instability (LDI) has tentatively been identified in the Nova gasbag experiments. LDI is the decay of the SRS electron plasma wave into another electron plasma wave and an ion-acoustic wave. LDI is identified by Thomson scattering ${ }^{2}$ off the ion acoustic wave. Figure 1a shows the geometry of the experiment, in which the $4 \omega$ Thomson scattering probe beam $^{6}$ and the SRS driving beam geometry are chosen to satisfy the k-matching condition with the LDI ion wave. The target plasma is a gasbag filled with $49.5 \% \mathrm{CH}_{4}$ $49.5 \% \mathrm{C}_{3} \mathrm{H}_{8}$, and $1 \% \mathrm{Ar}$, which is heated using nine of the Nova beams; this gives a density of $0.11 n_{c}$ (where $n_{c}$ is the critical density) for $0.53 \mu \mathrm{m}$, the wavelength of the SRS-driving beam (beamline 9) that has an intensity of $6 \times 10^{14} \mathrm{~W} / \mathrm{cm}^{2}$. Figures $1 \mathrm{~b}$ and $1 \mathrm{c}$ compare Thomson scattering streak records with and without the SRS-driving beam and show the appearance of the LDI signal after the heater beams have turned off. Measurements of the SRS reflected light levels show saturation, consistent 


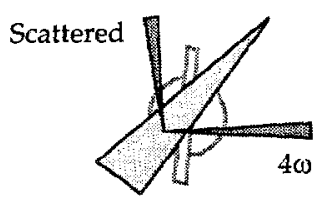

$t, \mathrm{~ns}$

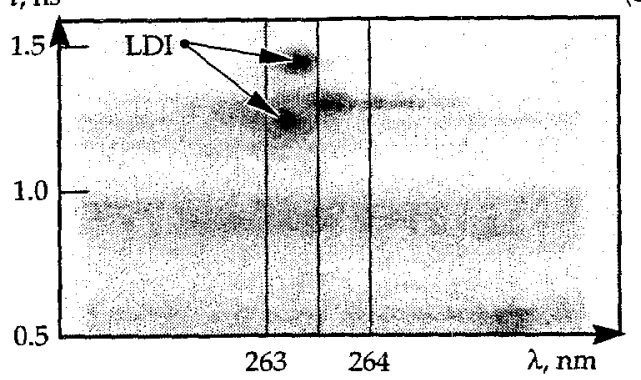

$t, \mathrm{~ns}$

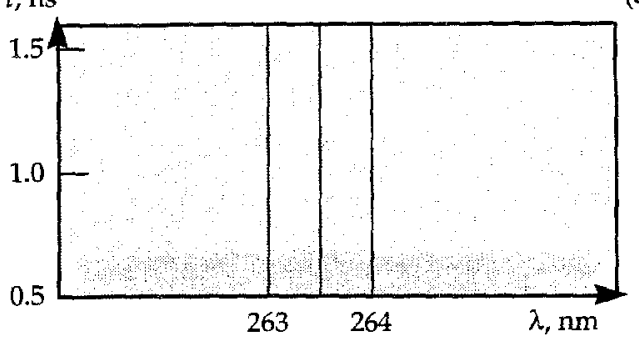

FIGURE 1. Results of Thomson scattering measurements of the LDI ion wave product from the decay of SRS electron plasma waves. (a) The probe and collection geometry. (b) Thomson scattering signals observed with a pump beam. (c) Measurements without a pump beam. (08-00-0500-2411pb01)

with the LDI saturation mechanism. This result is important for identifying the physical process to be modeled in F3D.

Thomson scattering measurements were also made on Nova gasbag experiments to quantify the fluctuation level of the SBS ion wave. In these experiments, nine of the Nova beams were used to form the plasma and the tenth was used to drive SBS. The geometry between the SBS-driving beam and the $4 \omega$ Thomson scattering beam was such that collective scattering from the SBS ion wave was obtained by the Thomson scattering detection system. Scattered signal from thermal plasma fluctuations was also collected after the driving beam turned off, allowing a quantitative measurement of the growth of the ion fluctuations as a result of the SBS instability. This was obtained for several different laser

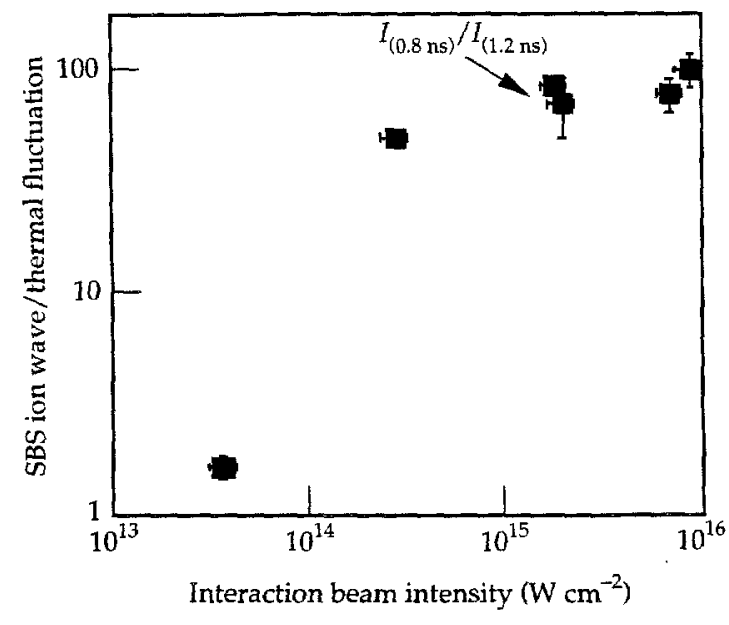

FIGURE 2. Measured SBS ion wave density fluctuations vs incident laser intensity. The fluctuation level is measured using Thomson scattering. (08-00-0500-2412pb01)

intensities (see Figure 2) so one can observe the onset of SBS saturation for laser intensities above $2 \times 10^{14} \mathrm{~W} / \mathrm{cm}^{2}$. This result is important because, from the known plasma conditions and the width of the Thomson scattering ion wave feature, one has the opportunity to identify the saturation mechanism. The measured fluctuation levels are also an important benchmark to test the physics modeled by F3D.

\section{Forward Scattered Light in Nova Gasbag Targets}

Forward-scattered light refers to the $351-\mathrm{nm}$ probe laser light that is transmitted through a plasma and emerges in a cone angle that is typically two to three times as large as the incident cone angle. We studied the character of forward-scattered light in long-scalelength gasbag plasmas for various plasma and laser conditions. A scatter plate and an array of optical and fiber detectors provided quantitative amplitude measurements of over 3 orders of magnitude in the forward-scattered light intensity. The forward light was characterized in terms of its angular spread, spectral content, and time history. Plasma conditions for the gas target varied in density from $7 \%$ of critical to about $15 \%$ of critical for $351-\mathrm{nm}$ light, and the ion damping ranged from 0.1 to about 0.4 . The plasma electron temperature ranged from about 1.8 to 
$2.8 \mathrm{keV}$. Probe laser light illuminated the target in an $f / 4.3$ or $f / 8.5$ cone with an intensity ranging from $0.7 \times 10^{15} \mathrm{~W} / \mathrm{cm}^{2}$ to $2 \times 10^{16} \mathrm{~W} / \mathrm{cm}^{2}$, and was smoothed with various combinations of KPP, SSD, and polarization smoothing (PS) schemes.

The transmitted light from an $f / 8.5$ probe beam illuminating a $7.5 \%$ critical gasbag plasma at $2 \times 10^{15} \mathrm{~W} / \mathrm{cm}^{2}$ with KPP smoothing only showed about $20 \%$ of the transmitted light outside of an $f / 4$ cone angle. The data corresponding to this case is shown in Figure 3. LASNEX calculations show that refraction can account for the majority of the light scattered between the $f / 8.5$ and $f / 4$ cone angles $\left(3^{\circ}\right.$ to about $\left.7^{\circ}\right)$. Light scattered beyond the $f / 4$ cone angle is due to beam spray from filamentation and/or probe-induced short-scale-length inhomogeneities in the plasma. The forward-scattered light amplitude measurements are time integrated. Time-resolved measurements show a somewhat larger angular spread early in the interaction, before the plasma has reached peak temperature, which then reduces at peak temperature. Application of SSD and PS reduce the large-angle scattered light a factor of 8 , but the effect on the small-angle scattered light is negligible. Amplitude measurements of the forward-scattered light from a $14 \%$ critical plasma at an intensity of $4 \times 10^{15} \mathrm{~W} / \mathrm{cm}^{2}$ show qualitatively similar results. A larger fraction $(30 \%)$ of the transmitted light is scattered beyond the $f / 4$ cone angle, and the scattered-light amplitude shows a slower falloff with angle. Application of SSD and

FIGURE 3. Discrete data points show the angular spread of the forwardscattered light for a $7.5 \%$ critical gasbag with KPP smoothing only and with $\mathrm{KPP}+$ SSD+PS. The solid lines show the results of the forward scattering model that uses a prescribed density fluctuation spectrum with amplitude $\langle|\delta n / n|>$. (08-00-0500-2415pb01)

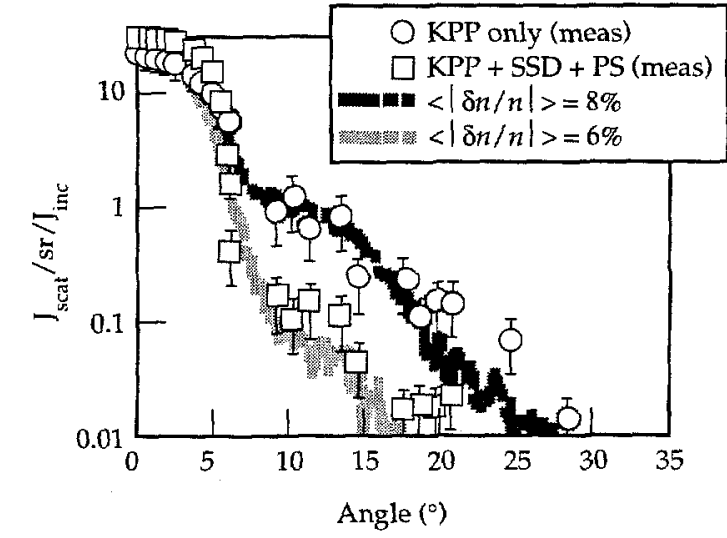

PS reduces the large-angle scattered light by a factor of 3 . Also shown in Figure 3 is the result of a model that calculates the forward scattering of laser light through a turbulent plasma. A prescribed fluctuation spectrum gives a calculated scattered light signal that agrees well with the measurement. The spectrum has scalelength components corresponding to background heater beam-driven fluctuations and filamentation/FSBS (forward stimulated Brillouin scattering) -induced fluctuations with an overall amplitude of $\delta n / n \sim 0.06$ with SSD and polarization smoothing.

Spectral measurements show a redshift and broadening of the forward-scattered light spectrum. The redshift increases with angle in agreement with the forward SBS dispersion. At intensities above $8 \times$ $10^{15} \mathrm{~W} / \mathrm{cm}^{2}$, measurements of the $351-\mathrm{nm}$ transmitted probe light show an enhanced redshift that is consistent with strongly driven FSBS. The spectral width (deconvolved with the instrument function) is typically $0.1-\mathrm{nm}$ for an intensity of $2 \times$ $10^{15} \mathrm{~W} / \mathrm{cm}^{2}$. This indicates that the laser-plasma interaction produces a laserinduced bandwidth or self-smoothing effect. The width increases with increasing intensity and with application of laser SSD.

In an effort to separate out the beam spreading due to intrinsic plasma inhomogeneities from the probe-induced spreading, we measured the beam spray from background inhomogeneities using a separate low-intensity probe laser. Forward scattering using a $263-\mathrm{nm}$ probe laser showed the presence of background inhomogeneities, which could account for small-angle spreading of the 351-nm probe but not large-angle spreading.

Measurements of angular spreading in higher ion damping $\mathrm{He} / \mathrm{H}_{2}$ targets at 7 to $8 \%$ critical density showed angular beam spread that was similar to the lower ion damping gas targets. Simulations of the forward-scattered light in a $\mathrm{He} / \mathrm{H}_{2}$ target using F3D show some agreement with the measurements. For example, the measured and calculated angular spreads agree out to an angle of about $9^{\circ}$. Beyond this angle, the calculation shows a more rapid falloff than the measurement. The calculated redshift and width of the forward-light spectrum agrees well with measurement at $9^{\circ}$ 
but not at $2^{\circ}$. We are continuing to use the measurements and simulations to better understand the physics of the forwardscattering process.

\section{LPI Experiments with $\mathrm{He} / \mathrm{H}$ Gas Mixture on Nova}

Cryogenic gasbag experiments on Nova were conducted to explore the dependence of SRS and SBS on low- $Z$ and higher ion acoustic damping plasmas with similar density and $Z$ as the plasma expected in the central region of a NIF hohlraum. Previous experiments by both LLNL and Los Alamos National Laboratory (LANL) scientists in room-temperature gas-filled targets produced data showing SRS increasing dramatically with increasing ion acoustic damping.

Figure 4 summarizes the scattered light level results from the cryogenic gasbag experiments and indicates that the SRS and SBS decreased with increasing ion acoustic damping. In addition, application of SSD showed a reduction in both the SRS and SBS of about a factor of 2 or more. Three gas mixtures consisting of different concentrations of $\mathrm{H}, \mathrm{He}$, and Ne provided nominal ion acoustic damping values of $0.1,0.3$, and 0.4 . The targets were cooled to $30 \mathrm{~K}$, pressurized to $1 \mathrm{~atm}$ with the particular gas mixture, and laser-heated like standard gasbag targets with 9 defocused Nova beams. The cryogenic gasbag was $25 \%$ larger in size compared to a typical gasbag in order to provide time for achieving higher ion temperature. The SRS spectra confirmed that the plasma density was in the range of 7 to $8 \%$ of critical for 351-nm light. Thomson scattering measurements showed a peak electron temperature that was estimated to be about 2.3 to $2.5 \mathrm{keV}$ in the center for the targets having $\mathrm{Ne}$ and about 1.8 to $2 \mathrm{keV}$ in the center for the targets without Ne. The SBS scattered light was redshifted by about $0.8 \mathrm{~nm}$; this is in agreement with the shift estimated from the ion acoustic dispersion relation. The SBS time-resolved amplitude at peak electron temperature decreased from $12 \%$ to less than $1 \%$ as the ion acoustic damping increased for the different gas mixtures. The SRS time-resolved amplitude at peak electron temperature decreased slightly from $11 \%$ to about $8 \%$ with increasing ion acoustic damping. We are presently conducting simulations of these results in an effort to understand them better.

\section{Modeling Developments}

The parallel code $\mathrm{pF} 3 \mathrm{D}$ is being developed to predict the backscatter, transmission, spreading, and deflection of NIF laser beams in ignition targets. Presently, pF3D contains physics modules that model light propagation, nonlinear 3D Eulerian hydrodynamics, and linearized nonlocal heat conduction, which means that presently the simulations include filamentation, beam bending, and forward SBS. Present computational capabilities allow the simulation of a plasma with the dimensions $225 \times 900 \times 2500 \mu \mathrm{m}$, which is approaching the size of a NIF beam. Preliminary simulations indicate that plasma self-smoothing of the beam is an important effect that needs to be modeled to better design laser smoothing concepts such as KPPs.

SBS backscatter from scale 1 hohlraums on Nova has been measured for a range of laser intensities and for SSD with a range of bandwidths. ${ }^{6}$ By postprocessing LASNEX simulation of the experiment with LIP and PIRANAH, the calculated spectral history has been shown to be in agreement with the data, with most of the SBS occurring near the hohlraum wall. F3D simulations showed that the nonlinearities in the acoustic wave evolution are important for

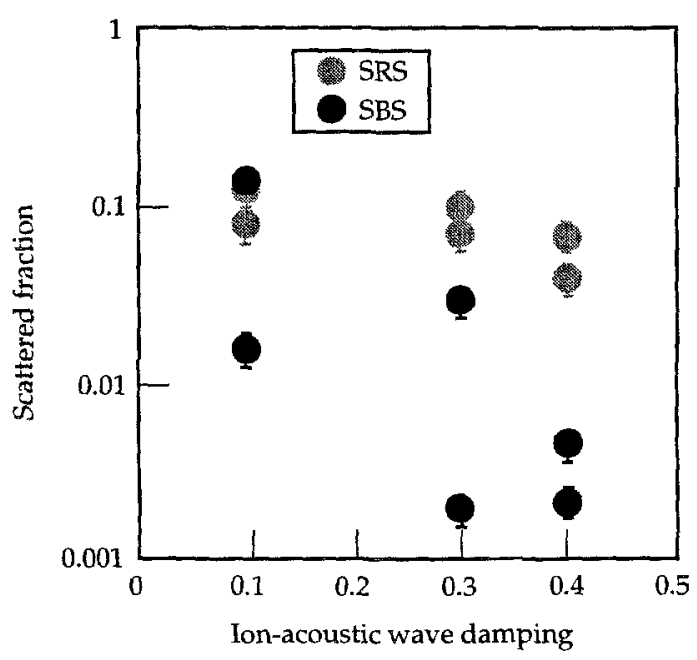

FIgURE 4. Plot showing that the SRS and SBS measured at peak temperature for the low-Z cryogenic gasbag targets decrease as a function of increasing ion acoustic damping. (08-00-0500-2416pb01) 
understanding the intensity dependence of the SBS evolution (see Figure 5). Most of the SBS occurs in hotspots with $I>3 I_{0}$, where $I_{0}$ is the average intensity. Simulations using $\mathrm{pF} 3 \mathrm{D}$ showed that the onset of plasma selfsmoothing in the range $2-4 \times 10^{15} \mathrm{~W} / \mathrm{cm}^{2}$ is important for understanding the intensity scaling of SBS.

\section{Notes and References}

1. B. MacGowan et al., Phys. Plasmas 3, 2029-2040 (1998).

2. S. Glenzer et al., Phys. Plasmas 6, 2117-2128 (1999).

3. D. Hinkel et al., Phys. Plasmas 6, 571 (1999).

4. E. Lefebvre et al., Phys. Plasmas 5, 2701-2705 (1998).

5. R. Berger et al., Phys. Plasmas 6, 1043-1047 (1999).

6. S. Glenzer et al., Rev. Sci. Instrum. 70, 1089-1092 (1999)

FIGURE 5. Results for polarization smoothing in scale 1 hohlraums using an $f / 8$ focusing geometry: (a) experiments and (b) comparisons to $\mathrm{F} 3 \mathrm{D}$ results.

(08-00-0500-2414pb01)
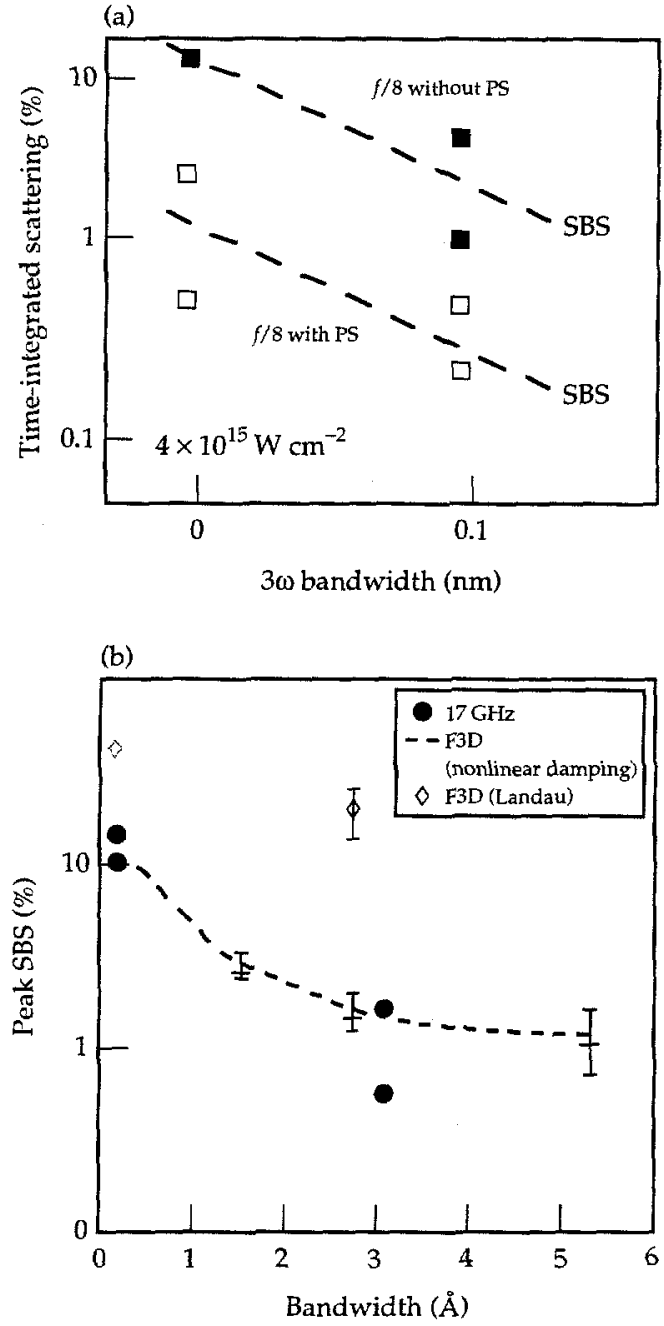

\section{Quantitative Analysis Tools for $p F 3 D$ Parallel Code}

Simulating laser-plasma interactions in a plasma volume approaching the size of an entire NIF beam is computationally demanding, requiring a massively parallel processor (MPP) to operate on a data set exceeding $100 \mathrm{~GB}$. The entire problem is decomposed into subdomains, which are parceled out to the processors of the MPP. At specified intervals during the simulation, each processor writes a state file containing all of the data for the local subdomain. Because reassembly of the physics data for the entire domain is intractable, individual quantities can be collected and analyzed to diagnose simulation results, either during the run or post facto. To facilitate this collection and analysis, an efficient tool has been developed.

Initially, the tool was designed to extract slices of laser intensity and electron density, but the tool has since been expanded to collect other hydrodynamic quantities as well. More recently, it can gather rectangular 3D blocks of data as well as planar slices. By implementing the tool in interpreter code, it can be used to make diagnostic plots either during the course of a simulation or in postprocessing.

Figure 6 was made using the data collection tool to gather the energy intensities distributed across multiple processors. A slice of laser intensity is taken through the center of the $3 \mathrm{D}$ beam after $22 \mathrm{ps}$ of simulation. The beam has 2 TW of laser power into an $f / 8 \lambda_{0}=351 \mathrm{~nm} \mathrm{KPP} \mathrm{with} \mathrm{Nova}$ aberrations. The interaction plasma is a $350-\times 350-\times 600-\mu \mathrm{m} 0.1 n_{\mathrm{c}} \mathrm{C}_{5} \mathrm{H}_{12}$ slab with $T_{\mathrm{e}}=3 \mathrm{keV}$. The simulation was performed on 256 processors of the ASCI Blue machine at LLNL using 33 GB of data, a much larger problem than the memory size of a single machine.

\section{Full-Size pF3D Simulation of Gasbag and Scale 1 NIF Plasmas}

This year we performed several "full-scale" pF3D simulations of Nova beams propagating through gasbag plasmas. We chose to model one of the recent cryogenic $\mathrm{H} / \mathrm{He}$ experiments for which 


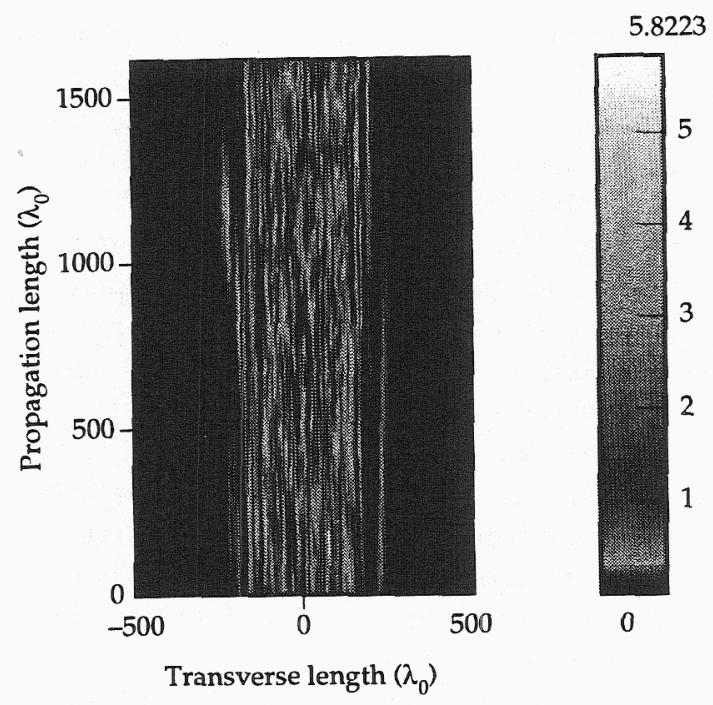

FIGURE 6. yz-slice of laser intensity showing the propagation of a KPP beam through a $350-\times 350-\times 600-\mu \mathrm{m}$ plasma slab. (Intensity is $1 / 2 \times 10^{15} \mathrm{~W} / \mathrm{cm}^{-2}$.)

(08-00-0500-2417pb01)

forward-scatter angular and spectral data were available.

These runs are our largest to date, using a Cartesian mesh with 1.3 billion zones and 512 processors of the ASCI IBM SP $/ 2$. The simulation volume of $700 \mu \mathrm{m} \times 700 \mu \mathrm{m} \times$ $2.6 \mathrm{~mm}$ enclosed the entire Nova beam cross section and the region of significant plasma density along the beampath. Full-scale NIF outer beam simulations will require approximately three times the memory of these runs and the use of the ASCI machine upgrade to become practicable.

The specifications of the $f / 4$ phase plate used in the campaign were combined with a beam aberration model. pF3D (with the latest beam package) then reconstructed the electric field of the laser spot on the gasbag target and propagated it self-consistently through the plasma. The plasma density, velocity, and temperature profile, along the beampath, were obtained from LASNEX simulations of the experiment and imported into $\mathrm{pF} 3 \mathrm{D}$. The simulations included not only the relatively constant density interior gas region but also the blast waves and the expanding exterior plasma.

Figures 7 and 8 are two pictures from the gasbag simulation. They show the near-field intensity of the incident and transmitted beams after $10 \mathrm{ps}$. The incident

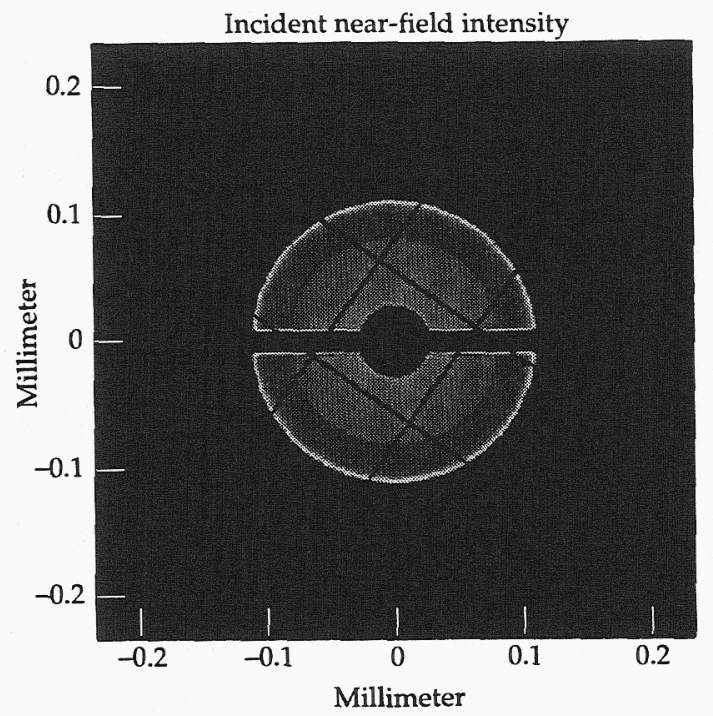

Figure 7. A gasbag simulation picture showing the near-field intensity of the incident beam after 10 ps.

(08-00-0500-2418pbo1)

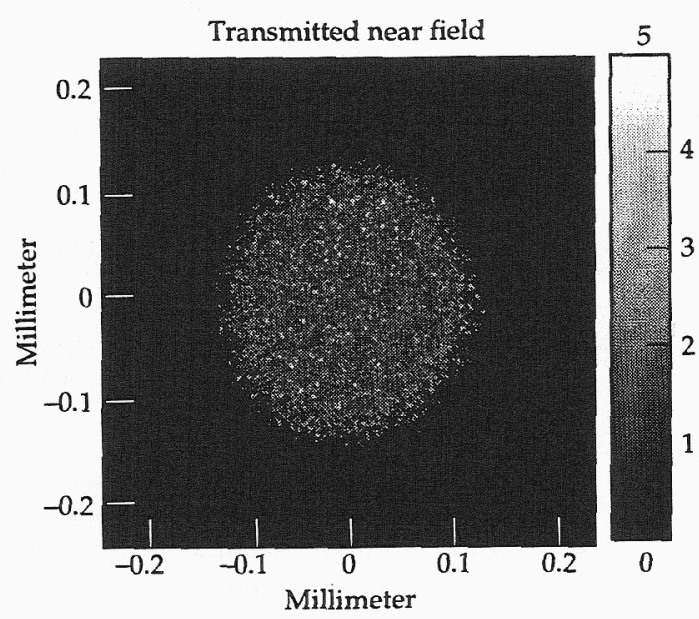

FIGURE 8. A gasbag simulation picture showing the near-field intensity of the transmitted beam after $10 \mathrm{ps}$. (08-00-0500-2419pb01) beam shows the beam stop and the splits in the KDP crystals. The transmitted beam shows considerable speckle structure and some spreading outside the original f-cone. In Figure 9 the calculated spreading is compared with the experimental measurements. Note that limitations on the spatial resolution of the simulation do not yet allow comparison with the data at larger angles of scatter than depicted. Lastly, in Figure 10 we compare the spectrum of the transmitted light inside and just outside the beam cone. The $p F 3 D$ frequency spectrum (broadened by the experimental resolution) agrees well with the data in both shift and broadening outside the lens cone (11-12 degrees), but shows a greater broadening 


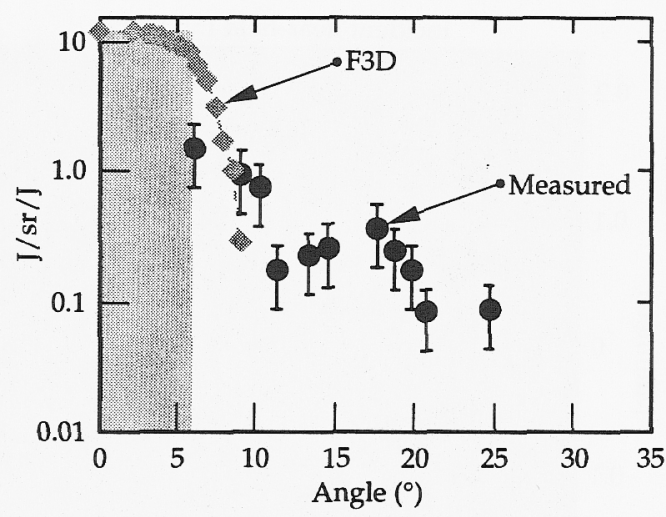

FIGURE 9. The calculated (F3D) beam spreading compared with the experimental measurements. (08-00-0500-2420pb01)

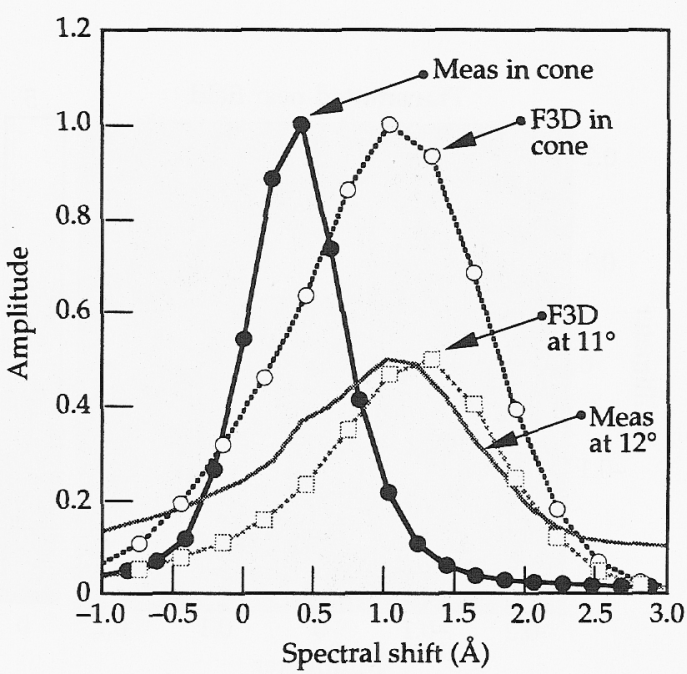

FIGURE 10. Comparison of the spectrum of the transmitted light inside and just outside the beam cone. The pF3D frequency spectrum agrees well with the data in both shift and broadening outside the lens cone (11-12 degrees), but shows a greater broadening and shift for the light within the cone. (08-00-0500-2421 pb01)

and shift for the light within the cone, which suggests we are overestimating the contribution of forward SBS to the smallangle scattering.

\section{Progress on Phase Plate Optimization}

The present database on phase plate smoothing and its effect on stimulated scattering in NIF-like conditions shows there is a possibility that the present kinoform phase plate (KPP) design for NIF can be further optimized to increase energy coupling efficiency. Last year's experiments showed evidence that the difference in scattering produced by different KPP and random phase plate (RPP) designs is slightly greater than can be explained by simply comparing the profiles of the averaged intensity and has greater shot-to-shot variation (Figure 11). This year we have performed calculations and experiments to determine what statistical properties of the focal spot can be controlled by the phase plate design and how these properties affect the scattering in NIFlike plasmas.

Calculations and Nova experiments have shown that the statistical properties of the intensity distribution can be varied in two important ways by adjusting the phase plate. Numerical simulations of the best focus intensity of a phase plate smoothed beam in vacuum have shown that the speckle size away from best focus can be adjusted in conjunction with the best focus spot size by modifying the phase plate design. More recent three-dimensional (3D) calculations show that the intensity distributions produced by phase plate smoothing in vacuum

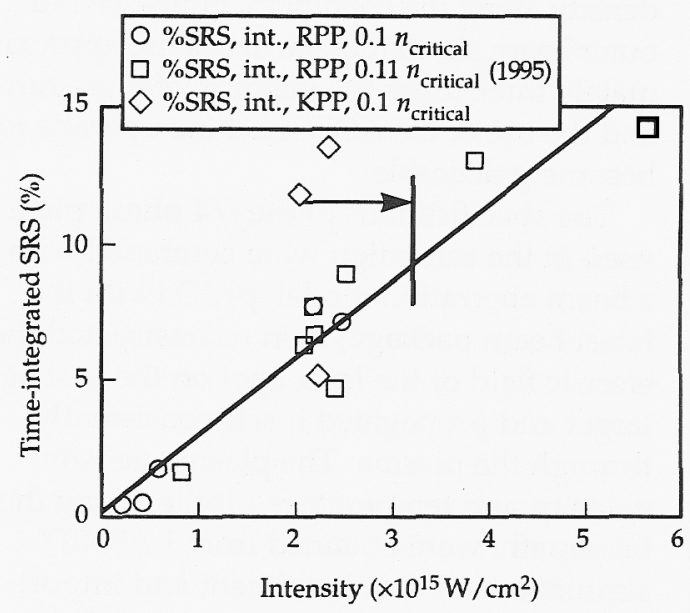

FIGURE 11. SRS reflectivities of an $f / 8$ beam with KPP and RPP smoothing and a $\mathrm{CH}$-filled gasbag plotted vs the peak envelope intensity. The vertical line represents the intensity at which the high-intensity part of the intensity distribution KPP is matched with the RPP case. This data suggests that KPPs produce slightly greater scatter with greater shot-to-shot variation. (08-00-0500-2408pb01) 
exhibit enhanced correlations on scales much greater than the specklesize ("blotches") and that the correlations can be controlled independently of the gross spot size by design of the phase plate. An example of this is shown in Figure 12, in which the beam profile produced with an RPP is compared to a Gaussian phase plate (GPP) that has a random phase with a Gaussian correlation function.

Experiments and numerical simulations of plasma instabilities have been performed to determine the effect of the speckle size and enhance correlations on scattering and filamentation. Analysis of experimental data comparing beams with equal intensities but differing intensity correlation lengths (or speckle sizes) has shown that scattering depends on the speckle size. In particular, in Nova experiments, decreasing speckle size reduced SRS by a factor of $>3$ in $7 \%$ critical $\mathrm{CH}$-filled gasbags, and increasing speckle size decreased SBS by a similar factor in $6 \%$ critical $\mathrm{CO}_{2}$-filled gasbags. Temporal and spatial profiles from some of these experiments are shown in
Figure 13. More recently, pF3D calculations of filamentation in NIF-like plasmas have demonstrated that the largescale correlations evident in "blotchy" beams produced greater filamentation and higher intensities to drive backscatter than comparable beams with a smooth envelope intensity. Further work on simulations and comparison with experiments is necessary to provide input to the design of the phase plate that minimizes the scattering in ignition experiments.

\section{PCDs on OMEGA and the Angular Distribution of $X$-Ray Power from the Laser Entrance Hole}

Measurement of the hohlraum flux is important for understanding the performance of implosion experiments. The flux is usually measured using Dante, which has a single view into the hohlraum through the laser entrance hole. Extrapolation of this measurement to a total flux requires
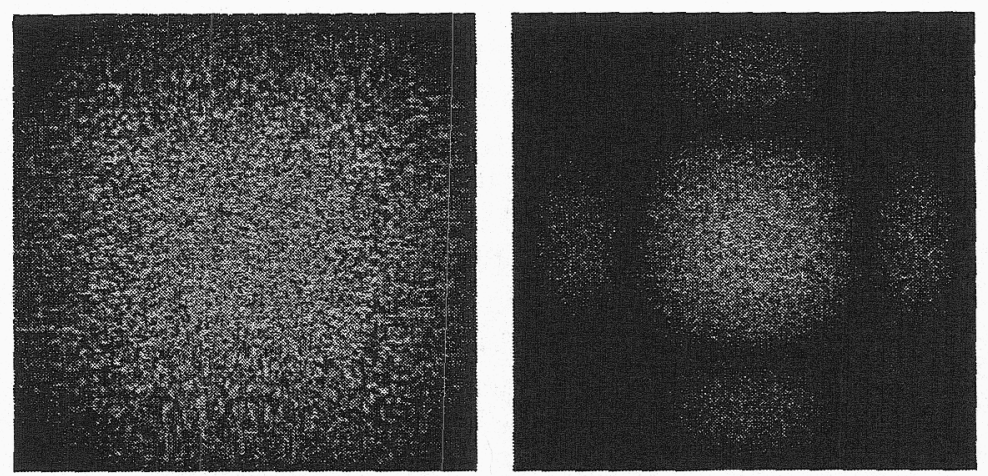

RPP

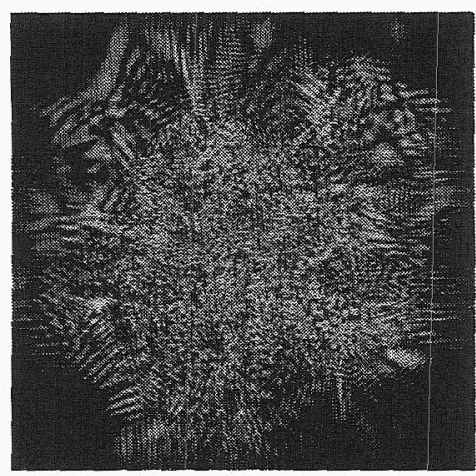

$z=5 \mathrm{~mm}$

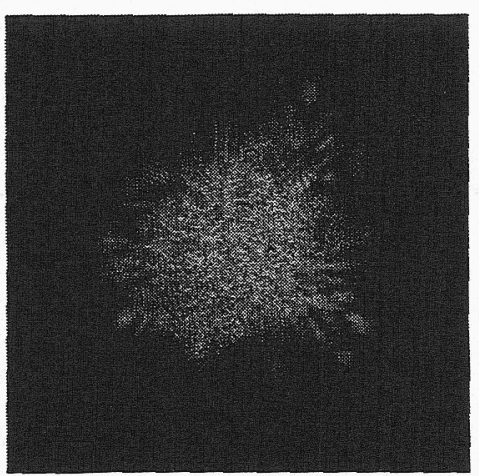

$z=0 \mathrm{~mm}$
FIGURE 12. Intensity profiles with two types of phase plate smoothing, RPP and GPP, show that large-scale correlations (blotchiness) can be affected by phase plate design.

(08-00-0500-2409pb01) 
FIGURE 13. Measurements of scattered power in NiF-like plasmas shows that beams with large speckle sizes (shown in black solid lines) have lower reflectivity than those with small speckle sizes (long and short dashed lines). Future phase plate designs can take advantage of any known dependencies on speckle size. (08-00-0500-2410pb01)
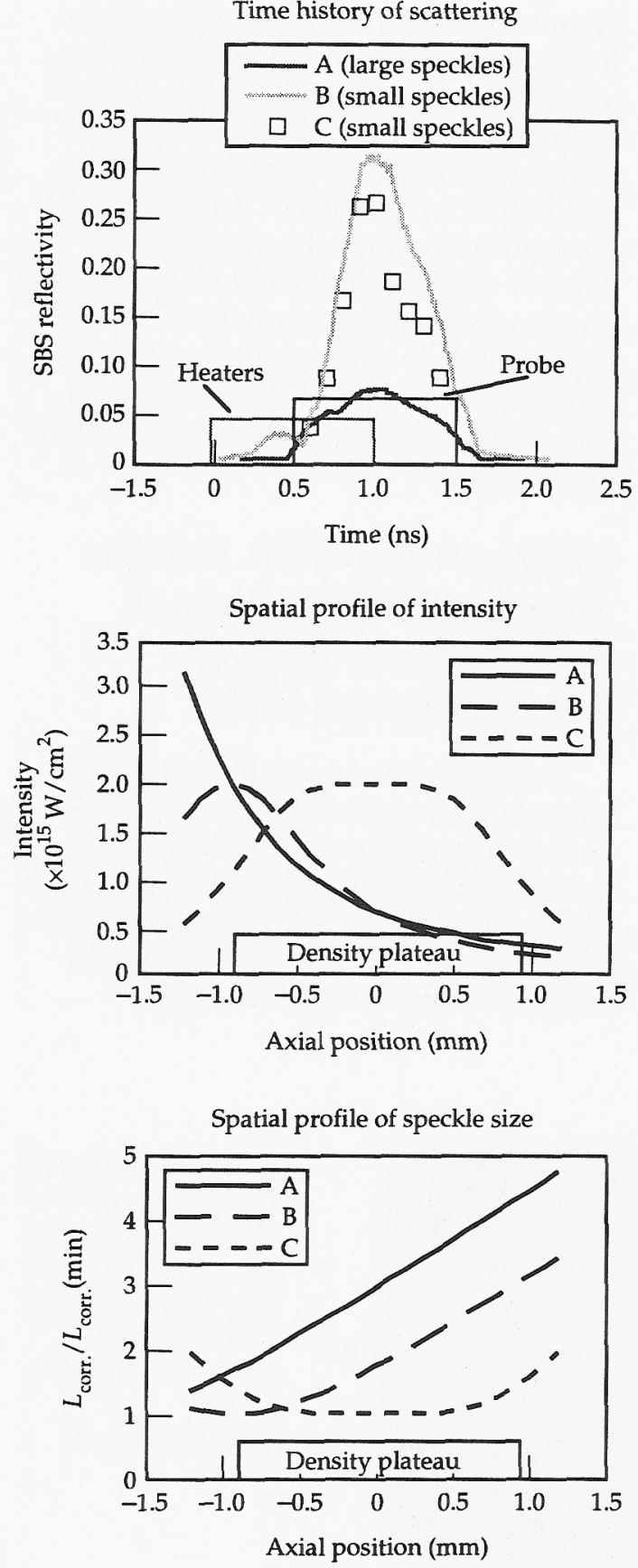

assumptions about the angular dependence of the flux that can be removed if additional detectors are positioned at different angles.

We have installed achromatically filtered PCDs (photoconductive detectors) made from natural diamond ${ }^{1-3}$ at several locations on the OMEGA chamber, including $\mathrm{H} 16, \mathrm{H} 11$, and a ten-inch manipulator (TIM)-mounted one in TIM-6. The signal is reduced by using an array of pinholes to cast multiple, overlapping images onto the detector. This reduces the flux some 500 times, while introducing no spectral distortion. The PCD-pinhole assemblies are cross-calibrated periodically to the Dante.

Figures 14 a-d show results from a half-raum experiment. The TIM-6 PCD is at 0 degree, the others are at 37 degrees and varying azmuthal angles, to the hohlraum axis. The measurements at 37 degrees show more flux than the measurement at zero degree, as expected, since the rear wall ( 0 -degree measurement) is heated indirectly, while the side walls (37-degree measurement) are directly heated by the laser beams.

\section{Notes and References}

1. D.R. Kania et al., J. Appl. Phys. 68, 124 (1990).

2. R. B. Spielman, W. W. Hsing, and D. L. Hanson, Rev. Sci. Instrum. 59, 1804 (1988).

3. D. R. Kania et al., J. Appl. Phys. 60, 2596 (1986).

\section{Hohlraum Symmetry}

Ignition of future high-convergence implosions at NIF will require accurate understanding, control, and measurements of hohlraum flux asymmetries. Several campaigns aimed at demonstrating symmetry control and measurement are being pursued at the 60-beam OMEGA facility. First, we are improving on the symmetry diagnosis techniques begun at Nova and extending them to NIF-scale hohlraum sizes at OMEGA. Second, we are utilizing the better NIF-like symmetry available at OMEGA to extend the convergence-ratio10 implosion studies started at Nova to convergence ratio 20 . This allows us to bridge the gap between understanding the performance of hydrodynamically equivalent Nova convergence-ratio-10 implosions and NIF convergence-ratio- 35 implosions. Finally, we are beginning to investigate in detail the sensitivity of various ignition designs to various asymmetry modes.

\section{Detection at OMEGA of $P_{6}$} and/or $P_{8}$ Asymmetry at NIF Foot Conditions

An experimental campaign investigating diagnosis and control of higher-ordermode hohlraum asymmetries during the 

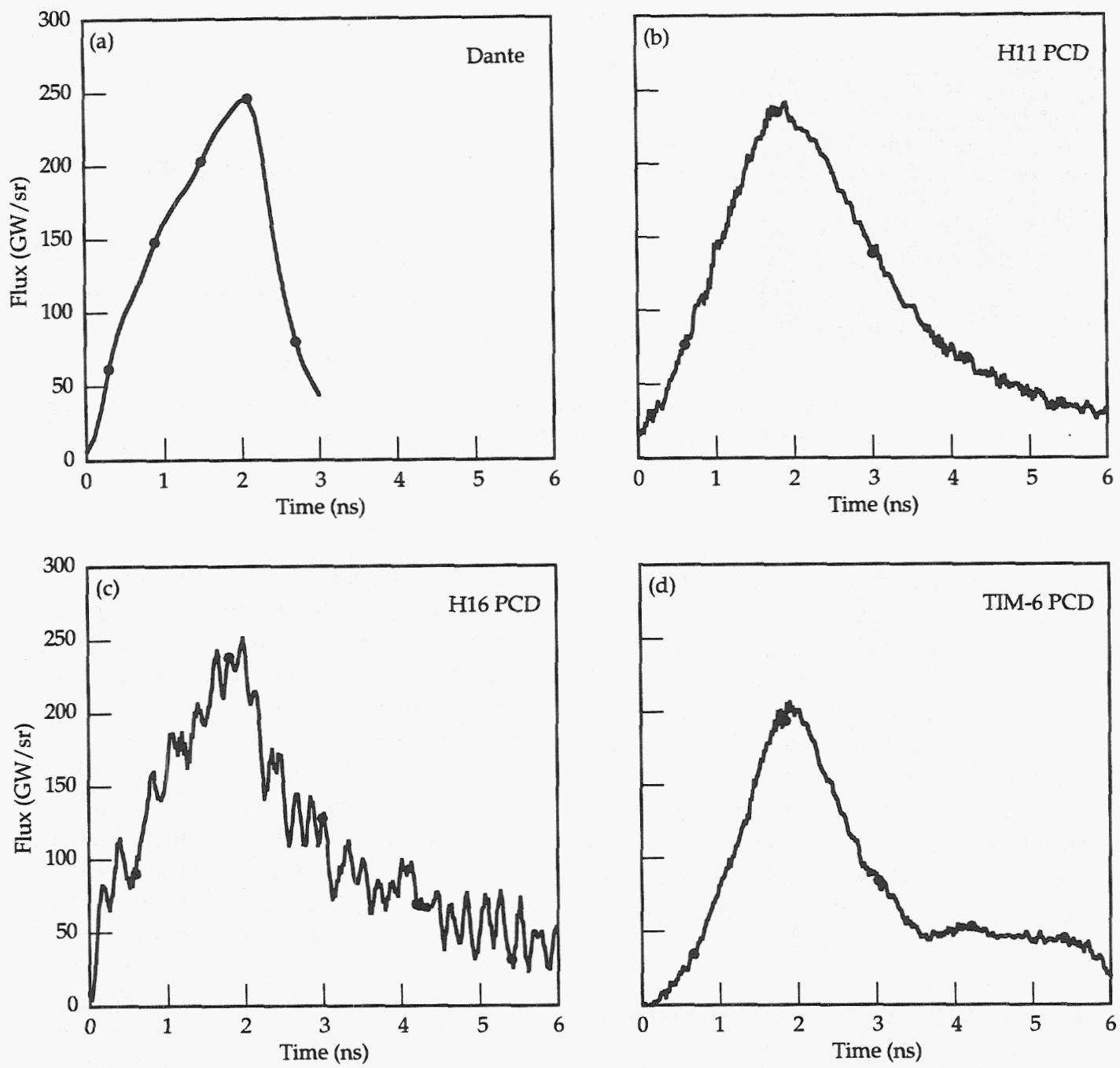

FIGURE 14. Dante (a) and PCD x-ray power measurements (GW/sr) (b-d) from a half-raum experiment, at various angles (see text). (40-00-1200-6346pb01)

foot (the first 8 ns of a 17-ns pulse) of the NIF ignition drive was begun this year at the OMEGA laser facility. The design used NIF-scale hohlraums $(4.8-\mathrm{mm}$-diameter by 8-mm-long) driven by a 7-ns, 75-eV drive using two sets of staggered OMEGA beams ( 42 beams in all). Flux asymmetries were inferred from the out-of-round distortions observed on backlit 1.5- to 3-mmdiameter $\mathrm{SiO}_{2}$ foam balls and Ge-doped plastic shells driven by such hohlraums. The larger shells were designed to enhance sensitivity to the higher-order asymmetry modes by reducing the case-to-capsule size ratio. The requisite large $(\approx 3-\times 3-\mathrm{mm})$ field-of-view backlighting was provided by a novel point projection scheme using backlit pinholes. ${ }^{1}$

Figure 15a shows a typical 200-ps-duration snapshot image of a backlit shell taken at $4.7-\mathrm{keV}$ photon energy. The high quality of the data stems from the reduction in noise achieved for any given asymmetry mode through a number of improvements: expanding the image size at the detector, using a point backlighter, eliminating film noise by switching to a charge-coupled device $(C C D)$ recording medium, and correcting for fixed-pattern camera noise. Figure $15 b$ shows the distortion between successive snapshots taken $4 \mathrm{~ns}$ apart as a function of polar angle. Some shots show a 
(a)

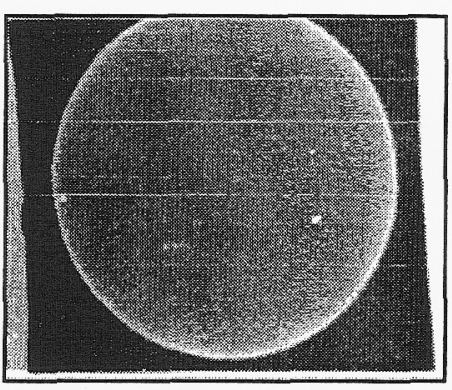

(b)

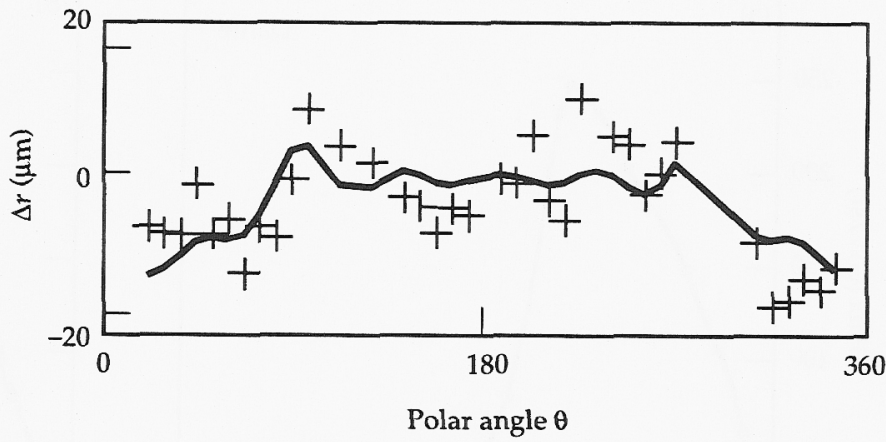

FIGURE 15. (a) Gated 4.7-keV backlit image of imploding 2-mm shell. (b) Distortion from round of shell limb vs polar angle, showing presence of higher order modes. (08-00-0500-2769pb01)

good shell distortion fit to a Legendre series about the hohlraum axis, suggesting we are indeed observing the effects of hohlraum flux asymmetries. However, odd modes are also visible, suggesting random imbalances due to the limited number of beams on at any given time $(\approx 20)$ and/or known misalignment. The larger shells provided 1- to 3- $\mu \mathrm{m}$ accuracy in Legendre coefficients $a_{0}$ through $a_{8}$. Of note, a 9- $\mu \mathrm{m}$ $a_{6}$ mode was observed, in qualitative agreement with preshot simulations.

\section{Improvement in Performance for Implosions with Convergence of 10-20}

A campaign of moderate- and highconvergence, high-growth-factor implosions continued at OMEGA using a NIFlike multiple-cone 40-beam hohlraum illumination geometry. The goal was to demonstrate improved implosion performance as a consequence of having improved symmetry over previous Nova hohlraums ${ }^{2}$ illuminated by a single cone per side and just 10 beams and over previous OMEGA hohlraums driven by staggered sets of beams. ${ }^{3}$

The OMEGA implosions were driven by a 10:1-contrast 2.5-ns-long pulse (PS26) in scale 1 ( $2.5-\mathrm{mm}$-long by $1.6-\mathrm{mm}$-diameter) hohlraums. The peak drive temperature was $190 \mathrm{eV}$, in good agreement with simulations including measured $10 \%$ backscatter losses during the peak of the pulse. The convergence ratio was progressively increased by reducing the initial capsule fill from $50 \mathrm{~atm}$ DD to $5 \mathrm{~atm}$ DD. Capsule fill integrity was improved by transporting later targets under pressure, decoupling from the gas source just before the shot. Capsule ablators consisted of smooth $1 \%$ Ge-doped plastic, with surface roughness between 0.02 and $0.05 \mu \mathrm{m}$ rms. The final cores were imaged in emission at $4 \mathrm{keV}$ with $21 \times$ magnification, and 5- $\mu \mathrm{m}$ resolution. The core shapes were close to round with residual distortions only $20 \%$ different from those calculated by LASNEX, translating to only a $1 \%$ offset between the experimental and calculated timeintegrated $P_{2}$ asymmetry.

Figure 16 plots the measured neutron yield compared to postshot simulations of the clean 1D yield versus convergence ratio inferred from the secondary neutron yield. The measured and calculated convergence ratios agree to $20 \%$. The moderate-convergence $\left(C_{\mathrm{r}} \approx 10\right)$ implosions at OMEGA perform repeatably at $65 \%$ of clean yield while the earlier Nova implosions performed at $25 \%$ of clean yield. Hence, we believe we have demonstrated the importance of improved symmetry in improving implosion performance.

Also shown on this plot are the results from higher-convergence implosions. The reduction in yield over clean yield at higher convergence is attributed to a combination of greater sensitivity of high-convergence implosions to asymmetry and residual 


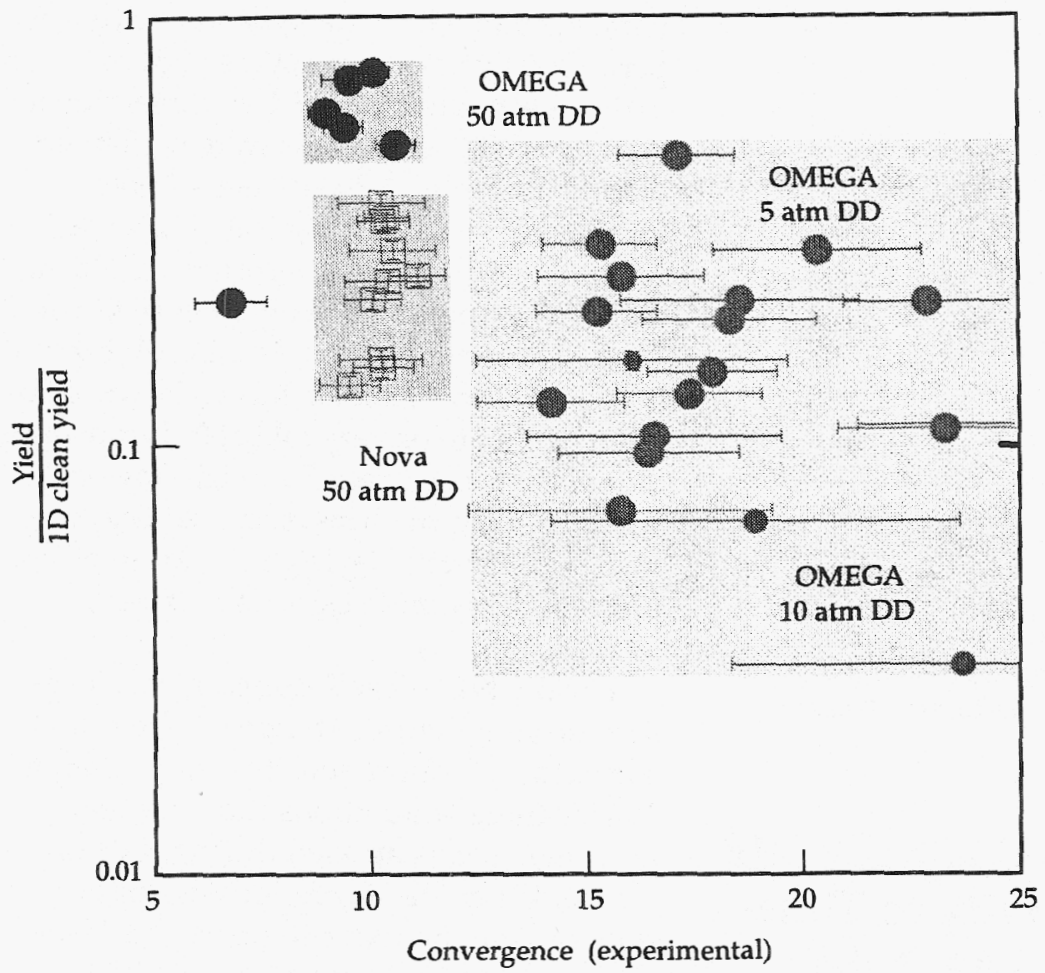

FIGURE 16. Ratio of measured to clean 1D calculated yield as a function of inferred convergence ratio for various fill capsules. (08-00-0500-2770pb01) capsule surface roughness. When including residual asymmetry and mix in the calculations, the 50 (10) -atm DD-fill capsules are calculated to perform within $90 \pm 10 \%$ $(40 \pm 10 \%)$ of predicted. Future campaigns will seek to reduce the scatter in the highconvergence performance by better preselection of capsules with lower surface roughness and better laser beam-to-beam balance.

\section{Experiments on OMEGA to Study Implosion Performance as a Function of Ablator Roughness for Capsules with Convergence of 10-20}

Besides the high-convergence smooth capsules shot as part of the campaign mentioned above, a series of six intentionally roughened 10 -atm DD-fill capsules were imploded. The rms surface roughness of these capsules was $0.3 \mu \mathrm{m}$, a factor of $10 \times$ greater roughness than for the smooth capsules. The surface roughness was imposed by laser ablating 60-100 randomly distributed pits. The measured core asymmetry of the imploded, roughened capsules varied greatly. The shot-to-shot core shape variation is attributed to the presence of shell spike growth from the surface-roughness-seeded RayleighTaylor instability, which quenches the fuel at random orientations and hence dominates the observed asymmetry.

The measured and calculated yields, including residual asymmetry and mix due to hydrodynamic instability growth of the surface roughness, are shown in Figure 17 normalized to the clean 1D yield. The 0.3- $\mu \mathrm{m}$ rms surface-roughness capsules show a clear reduction in yield relative to smoother capsules, demonstrating that in the better symmetry environment of the OMEGA hohlraums, we can isolate the effects of mix even for high-convergence implosions. For the rougher capsules (0.05- and 0.3- $\mu \mathrm{m}$ initial rms), the measured and predicted yields are in very good agreement, with a $10 \times$ yield degradation predicted by simulations due to mix alone for the roughest capsules. 


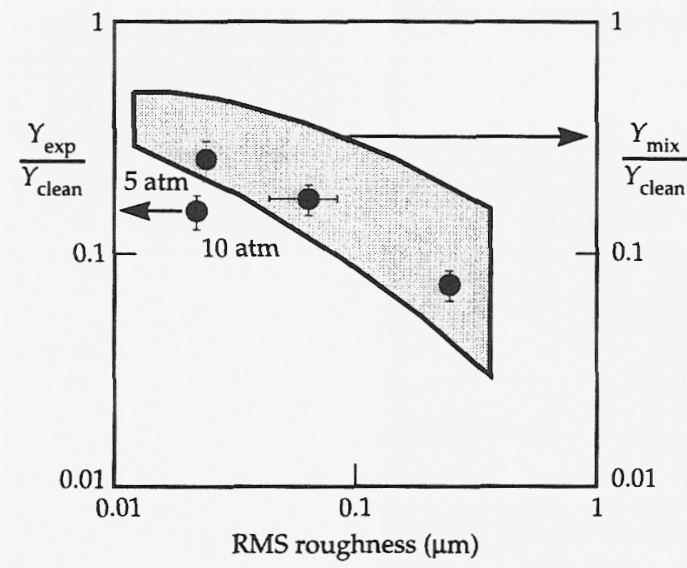

FIGURE 17. Measured and calculated yields including asymmetry and mix normalized to clean 1D yields as a function of initial capsule surface roughness. Points are averages over several shots. (08-00-0500-2771 pb01)

\section{Calculations to Quantify NIF Capsule Sensitivity to Time- Dependent Higher-Order Flux Asymmetries}

Although any high-order asymmetry modes at the hohlraum wall are strongly smoothed out at the capsule due to the stand-off distance between capsule and wall, the greater instability growth of perturbations seeded by these shorter wavelength modes can still make these modes problematic for NIF ignition capsules. Hence, a series of capsule-only LASNEX calculations were performed in which the radiation drive was perturbed by various amounts of pure $P_{6}$ and $P_{8}$ Legendre modes. ${ }^{4}$ Two-dimensional (2D) LASNEX simulations were performed on three capsules: a $300-\mathrm{eV}$ doped polystyrene capsule absorbing $150 \mathrm{~kJ}$; a $300-\mathrm{eV}$ polyimide capsule, also absorbing $150 \mathrm{~kJ}$; and a 250-eV copper-doped beryllium [Be( $\mathrm{Cu})]$ capsule absorbing $180 \mathrm{~kJ}$. The $250-\mathrm{eV}$ capsule is relatively small and, at this scale, would be expected not to be very robust (this capsule is also very susceptible to Rayleigh-Taylor perturbation growth, as discussed below in the subsection "Rayleigh-Taylor Analysis of Be and Polyimide Designs at 250-eV, 1.3-MJ Target").

For the NIF 300-eV doped plastic capsule design, Figures 18a and b show that the capsule is more sensitive to $P_{8}$ than to $P_{6}$ (for these simulations the radiation asymmetry was applied during the entire pulse). When the asymmetry is applied only during the foot (first $8 \mathrm{~ns}$ of a 17-ns pulse), the capsule can tolerate a higher level of asymmetry $\left(2 \% P_{6}\right.$ or $1 \% P_{8}$, as shown in Figures 18a and b) without significantly degrading the yield (assuming no other sources of yield degradation). The results for the $300-\mathrm{eV}$ polyimide capsule were very similar. However, Figure 18c indicates that the $250-\mathrm{eV} \mathrm{Be}(\mathrm{Cu})$ capsule is considerably more sensitive to the imposed asymmetry than are the 300-eV capsules. (a) $\quad P_{6}$ on for foot, entire pulse

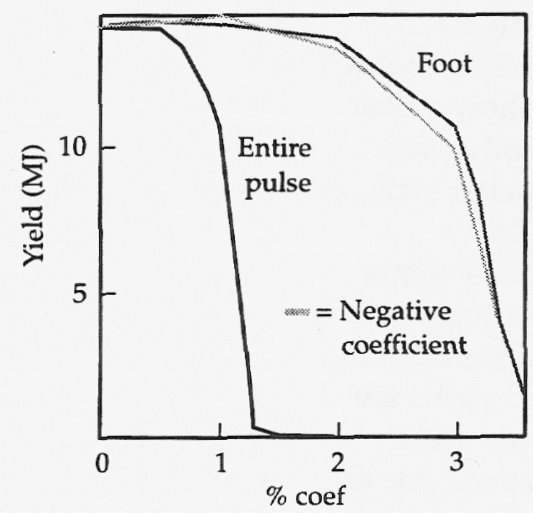

(b) $\quad P_{8}$ on for foot, entire pulse

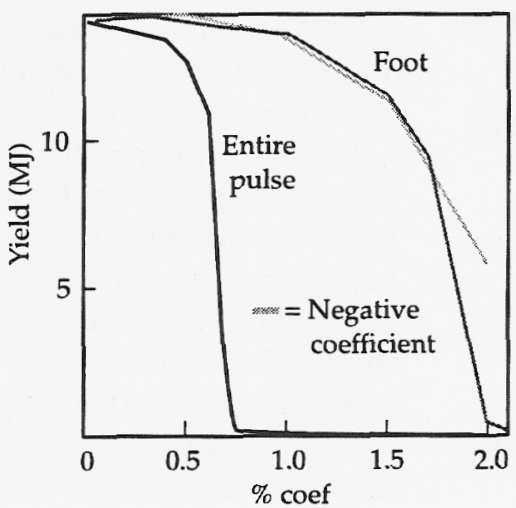

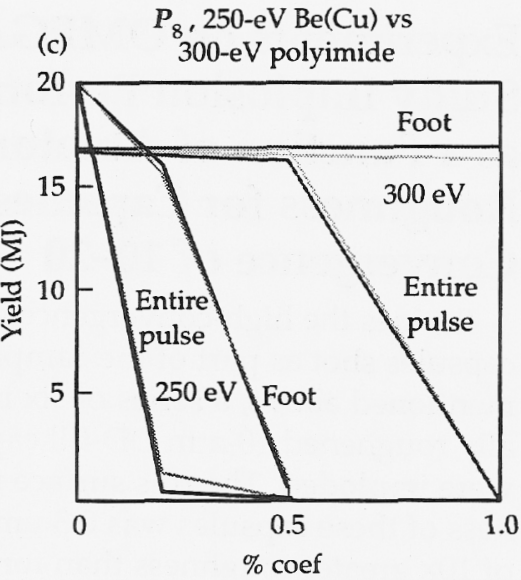

FIGURE 18. NIF ignition capsule yield as a function of imposed $P_{6}$ and $P_{8}$ flux asymmetry. (a) $P_{6}$ sensitivity for full pulse and foot for doped plastic capsule design, (b) $P_{8}$ sensitivity for full pulse and foot for doped plastic capsule design, $(c)$ comparison of $P_{8}$ sensitivity for foot and full pulse for 250-eV Be(Cu) and 300-eV polyimide design. (40-00-1100-6311 pb01) 
An input deck for doing 3D symmetry calculations of NIF hohlraums using the HYDRA radiation hydrodynamics code was also developed and tested. This entailed modifying HYDRA's laser energy deposition package. The deck will allow calculations of various hohlraum geometries during the NIF start-up phase, in particular for assessing the effect of azimuthal and random asymmetries for 96-beam NIF hohlraums.

\section{Notes and References}

1. O. L. Landen et al., Phys. Plasmas 2, 51 (1999).

2. S. G. Glendinning et al., Rev. Sci. Instrum. 5, 536 (1999).

3. R. E. Turner et al., Phys. Plasmas 2, 51 (2000).

4. O. S. Jones and S. M. Pollaine, Bull. Am. Phys. Soc. 43, 1896 (1998).

\section{Implosiom Optimization and Shock Timing}

An important part of the work leading to ignition is to specify the ablator dimension and composition for NIF ignition capsules and to determine the pulse shape required to drive the capsules. The basic choice of ablator material (beryllium with copper dopant and polyimide are two candidates) and dimensions will be optimized based on NIF performance and on target fabrication capabilities. An experimental campaign on NIF will be used to set the exact thickness and dopant concentration for the ablator, and the exact laser power as a function of time to drive it. Numerical simulations are not expected to be sufficient for this final tuning; we will need experimental procedures to make the final adjustments to both ablator and laser pulse shape necessary for ignition.

We made good progress on a number of these tasks during fiscal year 1999 (FY99). Several numerical sensitivity studies addressed NIF ignition campaign concerns: the differences between Planckian and nonPlanckian drive spectra, between two ablator opacity models, and between three DT equations of state. We explored two interesting ideas for measuring NIF capsule ablation rates; we designed a backlit implosion experiment at OMEGA to test one of these ideas next year. We also did simulations to determine the fabrication error in DT ice thickness that a NIF ignition capsule can tolerate. Three separate series of experiments used Nova to test NIF diagnostic techniques: First, in collaboration with the French Commissariat a l'Energie Atomique (CEA), we watched shock breakout and burnthrough of both beryllium and polyimide ablators, looking at drive scaling and copper dopant scaling (for beryllium ablators). Second, we did single-mode RayleighTaylor growth experiments, including sideon trajectory measurements, for both beryllium and polyimide ablators. Third, velocity interferometer for any reflector (VISAR) measurements of the shock propagating into liquid DD showed a stronger second shock overtake the first shock at NIF-like conditions. All of this computational and experimental work focuses on the need to design the ablator specification and shock timing procedures for the NIF ignition campaign.

\section{Sensitivity Studies of the 300-eV Polyimide Target to Drive Spectrum, EOS, and Opacity}

Sensitivity studies are focused on determining (1) the specifications for NIF shock timing and ablation rate diagnostics and (2) the sensitivity of different capsule designs on physics packages. The latter will determine if fundamental experiments on equation of state (EOS), opacity, or drive spectrum can increase our confidence in achieving ignition. Previously, we set rigid specifications for NIF shock timing diagnostics. Last year, we focused on determining ignition sensitivity to drive spectrum, ablator opacity, and fuel EOS, primarily for the $300-\mathrm{eV}$ polyimide (PI) $\left(\mathrm{C}_{22} \mathrm{H}_{10} \mathrm{~N}_{2} \mathrm{O}_{4}\right)$ target design. In addition, a preliminary estimate of the sensitivity of capsule performance on ablation rate and fuel thickness is reported.

Implosion simulations of our baseline polyimide target design comparing nonPlanckian to Planckian drive with the same flux show yields are very similar (18.8 MJ non-Planckian, 20 MJ Planckian). The ablation rate is the same in the two cases, contradicting indications from CEA simulations that the ablation rate is higher in polyimide with a non-Planckian drive. 
Preheat in unablated polyimide is the only significant consequence of the drive variation, so that the density is about $30 \%$ higher in the unablated polyimide when driven with a Planckian source. This would change the Rayleigh-Taylor instability growth but not have any other significant consequences.

As one estimate of opacity uncertainty in the $\mathrm{Be}(\mathrm{Cu})$ ablator, we have compared simulations with opacities from the HOPE opacity code vs the XSN code. The principal difference is that the ablation rate is $10 \%$ smaller with the HOPE opacities. Since most of the beryllium is ablated, the remaining payload mass is quite a bit larger with the HOPE opacities. The implosion velocity is accordingly reduced by about $15 \%$. All or most of this can be recovered by thinning the beryllium shell and possibly changing the concentration of copper dopant. An uncertainty this large in a NIF ignition campaign would be unacceptable, but a difference this large in ablation rate and implosion velocity would be readily evident in the ablator characterization experiments described below.

While our current program plan for shock timing does not depend on any credible errors in our knowledge of the EOS of capsule materials, the probability of success during the ignition campaign does depend weakly on EOSs. This is primarily because the shock timing requirements depend weakly on the fuel EOS. To estimate how the DT EOS uncertainties affect performance, we compare independent EOS models: (1) Q, the QEOS model that we have used for most of the NIF capsule design work, (2) S, the Sesame model favored by some Los Alamos designers, and (3) Y, a table to match the principal Hugoniot data collected by experiments at Nova.

Comparison is based on a $300-\mathrm{eV}, 1.3-\mathrm{MJ}$ polyimide capsule design. Fixing the capsule dimensions and materials, there are at least six variable parameters in the capsule pulse shape. The experimental plans for NIF shock timing will sort through all of these parameters and dimensions and locate the optimum, independent of the details of the EOS. Starting from the point design optimized for model $Q$, we varied a single pulse-shape parameter, running a series of five 1D simulations for each of the three EOS models. The parameter varied was the height of the foot pulse because the capsules are fairly sensitive to the foot level since it sets the strength of the first shock, and the three EOS models exhibit their greatest differences in the principal Hugoniot. The optimum for this parameter is essentially the same for all three, as Figure 19 shows. All three ignite and burn robustly at the optimum foot level giving nearly equal yield. The $Q$ and $S$ models exhibit nearly identical sensitivity to the foot level, while the Y model is substantially less sensitive to foot level.

The principal Hugoniot curves for the three models are shown in Figure 20; the first shock level is close to the region of maximum disagreement. However, the apparent decrease in sensitivity of model $Y$ to foot level is largely due to the fact that it predicts substantially lower pressure during ignition than the other models. This lower pressure at ignition may not be real. The difference is shown in Figure 21.

The width of the narrower $Q$ and $S$ tuning curves corresponds to about $\pm 10 \mathrm{eV}$. With our current NIF experimental plans, we will be able to find and correct errors to about $1 \mathrm{eV}$. If the DT EOS were closer to $Y$ than $Q$ or S, ignition on NIF would be somewhat easier but the experimental sequence on NIF would remain the same.

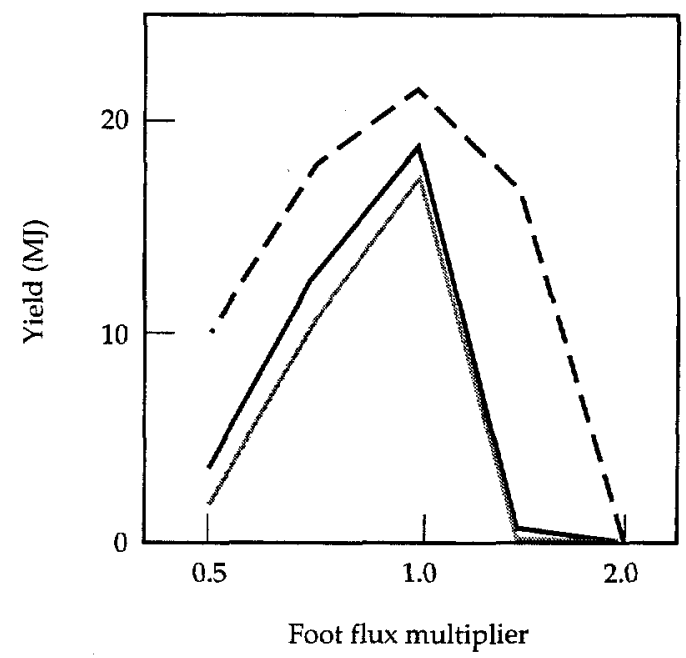

FIGURE 19. Capsule sensitivity to foot level for three different DT EOS models: $Q$ (black), $S$ (gray), $Y$ (gray dash). (08-00-0500-2763pbo1) 


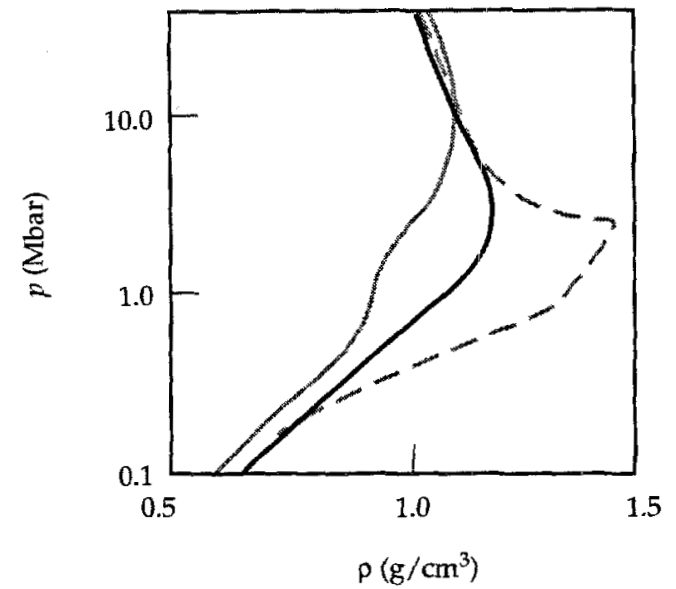

FIGURE 20. Principal Hugoniot for three DT EOS models: $Q$ (black), $S$ (gray), Y (gray dash). (08-00-0500-2764pb01)

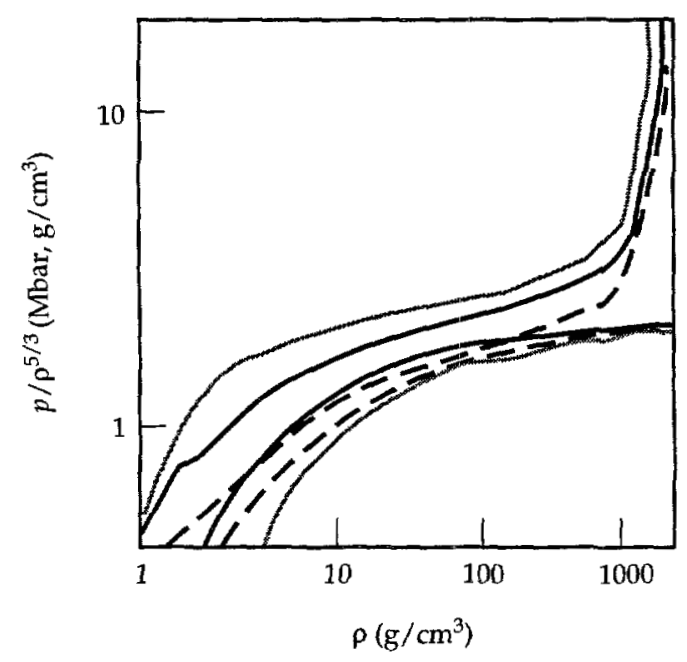

FIGURE 21. Thermodynamic trajectories of DT ice particles with three EOS models: $Q$ (black), $S$ (gray), $Y$ (gray dash). The lower curves are pressure at zero temperature; the dashes show the Fermi pressure. (08-00-0500-2765pb01)

Determining the correct ablator dopant, dopant profile, and ablator thickness are important milestones in the NIF ignition plan. In a polyimide $300-\mathrm{eV}$ design, ablation burns off $\sim 90-95 \%$ of the ablator. If the ablator completely burns off, $x$-rays preheat the fuel. If the ablator burns off too little, implosion velocity is reduced. The standard way for measuring ablation rate is to measure a burn-through foil in planar geometry. While we do not yet have a careful determination of the burn-through specification, there is a change in the hydrodynamics of an implosion when the burn- through varies by about 60 ps. For a planar experiment, variation in peak drive of $5 \%$ changes burnthrough time by $60 \mathrm{ps}$.

Because of this high sensitivity to burnthrough rate, we need a way to select the ablator thickness independent of simulations, in the same sense that our plan for defining pulse shape is independent of simulations. We explored two experimental techniques for choosing the ignition capsule ablator thickness. The first technique relies on imaging high-pressure, non-cryogenic capsule implosions, and a second relies on tracking a tracer layer in the ablator. In the high-pressure capsule, the ablator thickness is determined by how much burns off during the main part of the pulse, which ought to depend only weakly on the capsule fill or the pulse shape during the foot. We investigated the hydrodynamics of ignition capsules shot at room temperature, both DT and $D_{2}$ fills, and with pulse shapes that both included and omitted the foot of the fully optimized pulse shape. Without the foot, the convergence ratio of these highpressure gas capsules is under $4: 1$, which holds great promise for performing useful ablator experiments even with rather poor hohlraum symmetry (that is, before the symmetry campaign has produced highly symmetric designs). With the full NIF ignition pulse shape, ${ }^{1}$ the convergence ratio increases to a little over $5: 1$. The sensitivity of the convergence ratio to ablator thickness for the truncated pulse is shown in Table 1 for a polyimide $300-\mathrm{eV}, 1.3-\mathrm{MJ}$ design with initial fuel-mandrel radius of $965 \mu \mathrm{m}$ and initial mandrel thickness $15 \mu \mathrm{m}$.

TABLE 1. Sensitivity of the convergence ratio to ablator thickness for the truncated pulse discussed in text.

\begin{tabular}{lcc}
\hline $\begin{array}{l}\text { Ablator } \\
\text { thickness }(\mu \mathrm{m})\end{array}$ & $\begin{array}{c}R_{\min } \\
\text { (fuel-mandrel) }\end{array}$ & $\begin{array}{c}R_{\min } \\
\text { (mandrel-ablator) }\end{array}$ \\
\hline 90 & 346 & 486 \\
110 & 310 & 419 \\
130 nominal & 279 & 366 \\
150 & 251 & 318 \\
170 & 235 & 273 \\
\hline
\end{tabular}


Clearly the convergence ratio [thus $R_{\min }$ (fuel-mandrel) and $R_{\min }$ (mandrelablator)] is extremely sensitive to the ablator thickness. It is still unclear whether this type of capsule is a close enough analog to the cryogenic capsules to be used for ablator thickness selection, but the idea appears promising.

Preliminary design work has also been done on measuring several ablator features in spherical converging geometry using tracer layers. The notion is to heavily dope the inner part of the shell, for example, with germanium. The shell is backlit and observed at a frequency optimized to see the interface between the doped and undoped regions at the time of interest. With relatively high backlighter energy, there is the possibility of seeing when the shocks pass from the undoped to doped material, by seeing when the doped material starts to move. For NIF this will probably require quite high energies (possibly $12-\mathrm{keV}$ backlighting) and high resolution to see the relatively small motion resulting from the shocks. This measurement would be valuable for confirming that the incident flux on the capsule is the same as on the planar shock-timing experiments. The latter would still be necessary to ensure proper timing of the shocks in the fuel, while the spherical experiment ensures that the initiation of the shocks in the ablator is the same for the capsule in the center of the hohlraum as expected from the planar experiments. With lower backlighter energy, we would expect to see the more gross motion of the shell and doped layer, first seeing it accelerate inward and then, when the ablation front reaches the doped layer, seeing the dopant interface accelerate outward away from the imploding shell. It would give us a good measurement of the ablation rate, in the relevant spherical configuration. This measurement requires less precision and lower backlighter energy-perhaps $5 \mathrm{keV}$ on NIF, with $5 \% \mathrm{Ge}$ dopant. These concepts will be tested next year at OMEGA.

Finally, germane to all capsule designs is the sensitivity to the DT ice thickness. Initial thoughts were that if the first shock needed to be tuned to within $200 \mathrm{ps}$, then for a $\sim 1-\mathrm{Mbar}$ shock in the DT, the ice thickness needed to be known to $20 \mu \mathrm{m} / \mathrm{ns} * 0.2 \mathrm{~ns}$ $\sim 4 \mu \mathrm{m}$. We ran a 1D LASNEX simulation, which reveals that the cliff in performance for a 250 -eV design with 80- $\mu \mathrm{m}$-thick DT layer in a 2.2-mm-OD, 140- $\mu$ m-thick $\mathrm{Be}+\mathrm{BeO}$ capsule occurs when the DT thickness is $\pm 5 \mu \mathrm{m}$. This result is very different than the $\mathrm{Be}(\mathrm{Cu}) 300-\mathrm{eV}$ design where DT layer thickness of $58 \mu \mathrm{m}$ and $112 \mu \mathrm{m}$ both ignite. Both results are correct; the $\mathrm{Be}(\mathrm{Cu}) 300-\mathrm{eV}$ capsule has considerable margin to begin with, and the mode in which it ignites changes completely as the ice thickness varies, but it manages to ignite robustly in all these different ways. This capsule is sufficiently robust to ignite even with the relatively poor adiabat that results from this large change in shock timing, at least for these 1D simulations in which everything else is assumed to be perfect. Since we cannot avoid all of the other possible uncertainties, the specification of DT ice layer thickness has been set to a couple microns to ensure that the implosion is reproducible and robust with respect to other possible failure modes.

To extend our understanding of ablator performance (i.e., preheat effects, ablation rate, implosion velocity, etc.) and to develop new diagnostics for characterizing ablators to NIF specifications, we have begun a series of experiments on the OMEGA Laser facility. The initial design effort was described at the beginning of this subsection, and here we describe a proposed OMEGA indirect-drive backlit implosion prototype experiment that focuses on determining the ablation rate in spherical geometry. The capsule has a $5 \%$ titanium dopant in the innermost 5 to $9 \mu \mathrm{m}$ of a capsule, with about $25 \mu \mathrm{m}$ of undoped $\mathrm{CH}$ completing the shell. Simulated transmission with an iron backlighter is shown in Figure 22. The outermost drop in transmission tracks the outer edge of the doped layer, while the bulk of the imploding shell is visible as the minimum in transmission. When these two features separate, the doped layer is being ablated.

\section{Notes and References}

1. John D. Lindl, Inertial Confinement Fusion: the Quest for Ignition and Energy Gain Using Indirect Drive (Springer-Verlag, New York, NY, 1998). 


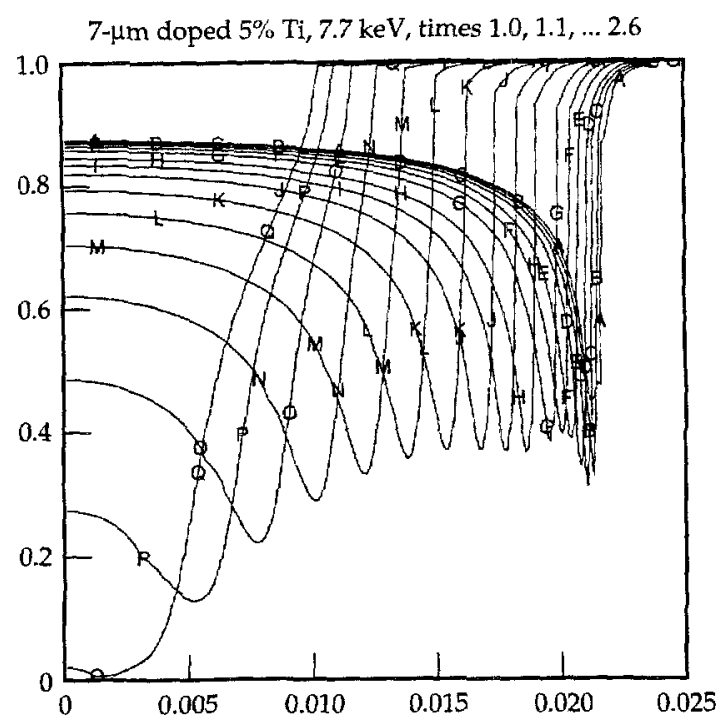

FIGURE 22. Transmission vs radius for a Ti-doped implosion. (08-00-0500-2766pb01)

\section{Nova Experiments Measuring Burn-Through and Shock Breakout of $\mathrm{Be}(\mathrm{Cu})$ and Polyimide Ablators}

We conducted experiments to test our modeling of prospective ablator materials for NIF ignition designs. This work examined Cu-doped Be and polyimide. All targets were hohlraums with multiple foils mounted over a slot, which also had an open gap for direct view into the hohlraum for timing and intensity normalization. Diagnostics measured $x$-ray drive (Dante), shock breakout (streaked optical pyrometer - SOP), and streaked x-ray burn-through. The $\mathrm{Be}(\mathrm{Cu})$ shots used 1-ns square pulses, while the polyimide shots used 2-ns square.

We examined drive scaling for different thicknesses of nominally $1 \% \mathrm{Cu}$-doped Bè. The hohlraums were scale 1.3,1, and 0.75 . The measured shock arrival times are in general agreement with modeling. The absolute brightness of the SOP signals is not well understood. The data generally show less contrast between preshock/ shock/burn-through brightness levels than predicted. Preshock signals indicate either greater than expected preheat or some experimental background.
Experiments to determine burn-through rates vs $\mathrm{Cu}$ concentration used $\mathrm{Cu}$ fractions $1.3 \%, 3.7 \%$, and $4.7 \%$, all in scale 1 hohlraums. The timing of the burnthroughs for the 3.7\%, 22- $\mu \mathrm{m}$-thick and $4.7 \%, 16-\mu \mathrm{m}$-thick foils are in approximate agreement with simulations, but the burnthrough of the $1.3 \%, 32-\mu \mathrm{m}$-thick sample is later and weaker than predicted. That sample burns through near the end of the drive pulse. The $x$-ray burn-through signals from a spectral band centered at $475 \mathrm{eV}$ rise much more slowly than predicted by simulations. The only factor that has been identified to give this effect in simulations is much greater-thanexpected leakage of energy from higher orders of the grating used to select the spectral band.

At $700 \mathrm{eV}$, burn-through had a more rapid temporal rise than at $475 \mathrm{eV}$, as expected. The burn-through of the $3.7 \%$ and $4.7 \%$ samples in the $700-\mathrm{eV}$ band agrees well with modeling. The $1.3 \%$ sample again burned through later and more weakly than expected. The sample burnthrough signals are lower than expected relative to the peak of the reference signal. Gold opacity is high at $700 \mathrm{eV}$, so it is possible that gold obscuration could have caused the early drop in the reference signal, the delayed burn-throughs, and the lower-than-expected burn-through signals. Reference signals at $475 \mathrm{eV}$, where the gold opacity is lower, remain high until after the end of the laser pulse.

Two polyimide burn-through shots were performed in August 1998 with samples of 23- $\mu \mathrm{m}$ and 26- $\mu \mathrm{m}$ thickness. The burn-through profiles are in good agreement with simulations using the drive calculated with LASNEX.

\section{Nova RT Single-Mode Experiments on $\mathrm{Be}(\mathrm{Cu})$ and Polyimide}

Rayleigh-Taylor (RT) growth measurements and 1D side-on measurements are sensitive integrated tests of our predictive capability of ablator performance. As such, we performed these experiments on both polyimide and beryllium doped with a 
few percent copper. In all cases, sample foils contained preimposed single-mode $(\lambda=30,50$, and $70 \mu \mathrm{m})$ sinusoidal perturbations. The experiments used scale 1 Nova hohlraums (Au cylinder $3000 \mu \mathrm{m}$ long, $1600 \mu \mathrm{m}$ diameter, with 1600- $\mu \mathrm{m}$ diameter laser entrance holes), driven with the Nova pulse shape "PS35," which was 3.5 ns long with nominally $4: 1$ contrast.

Measured polyimide side-on trajectories agree well with simulations. However, the measured modulation growth at all wavelengths is significantly reduced as compared to simulations. A larger-thanexpected high-energy component in the molybdenum backlighter spectrum can produce reduced contrast uniformly throughout the measurement interval. With a time zero contrast reduced by a factor of 0.66 , all three wavelengths can be made to agree with simulations. Offline $x$-ray fluorescence demonstrated that the elemental composition of the polyimide was as specified, and that the filter (Rh on a polyester backing) was Rh. Offline radiography determined the polyimide attenuation to $x$-rays at about $2 \mathrm{keV}$ was as anticipated. Diagnostic checkout shots, normally done with gold disks, were shot with Mo disks to remeasure the Mo spectrum and to measure the attenuation of polyimide patches placed at the gated microchannel plate detector. The Mo spectrum appears to be the same as that used initially to check the $t=0$ contrast. None of the shots gave a successful measurement of the polyimide patches. The possibility remains that the Mo spectrum has a greater high-energy component than measured; if the energy above $3 \mathrm{keV}$ is multiplied by five, the predicted contrast is reduced to the required $60 \%$ of the nominal value. We are in the process of checking the backlighter spectrum, filter transmission, and time zero contrast to determine if this effect is the cause of our discrepancy or if perhaps the difference between data and simulation is due to preheat.

For the $\mathrm{Be}(\mathrm{Cu})$ experiments, two sideon measurements of the trajectories are somewhat faster than simulated trajectories. We have examined the effect of varying the foil density, copper concentration, EOS, opacity, and drive. Varying the copper concentration within the uncertainty had little effect on the side-on trajectory. Several models for the $\mathrm{Be}(\mathrm{Cu})$ EOS were examined. Two available tables (Sesame and scaled EOP) gave similar results. Both were slower than the side-on trajectory obtained using QEOS. Drive models obtained with the albedo-corrected Dante and using the PCD data shifted by -150 ps gave similar results for the side-on trajectory. For nominal XSN opacities, the side-on trajectory is insensitive to a $10 \times$ increase in preheat.

Results of the simulations are sensitive to plausible variations in the opacities. For example, a global opacity multiplier of 0.8 brings the side-on into agreement with the data. With a global multiplier of 0.8 applied to XSN opacities, the increased drive penetration reduces the foil average density and reduces the growth of a perturbation, which is not consistent with the face-on data. Simulations of single-mode perturbation growth performed using nominal XSN opacities predict somewhat less growth and earlier burn-through than was measured. When opacities calculated with HOPE are used, reasonable agreement with measured perturbation growth is obtained for all wavelengths. The sideon trajectory is only slightly slower when HOPE opacities are used. The most important difference in the opacity appears to be the difference in the calculated copper edge below $0.1 \mathrm{keV}$. It is plausible that the detailed configuration accounting with average atom wave functions used in HOPE gives a better calculation of the opacity than XSN.

\section{Nova VISAR Shock Timing Experiments}

To determine the limits of the VISAR diagnostic for shock timing and to determine how well we model the complicated shock structure expected in shock tuning campaigns, we performed several different double-shock experiments and carefully compared the results with LASNEX simulations. We find that we can measure shock velocities with hohlraum drives up to the third shock level in a NIF ignition capsule, and we have accurately detected shock coalescence. Simulations of the 
results are very sensitive to details of the drive profile.

A variety of different drive levels were used on two basic pulse shapes: a "2-ns square" pulse and a 7-ns "PS100" pulse. Simple drive estimates suggested we would be examining drives in the range of 100 to $140 \mathrm{eV}$; the VISAR data indicate that one shot was probably driven above $150 \mathrm{eV}$. The "2-ns square" pulses produce a single decaying shock in the liquid $\mathrm{D}_{2}$ sample. The "PS100" pulses produce a first shock, which is later overtaken by a stronger second shock. The mechanism for the second shock is as follows: The first shock propagates through the aluminum ablator and breaks into the $D_{2}$, launching the first shock and causing a rarefaction to propagate back through the aluminum toward the ablation front. For "PS100," the drive is still on when the rarefaction reaches the ablation front; it reflects as a second shock wave, which propagates back through the aluminum, breaks into the postshock $\mathrm{D}_{2}$, and finally becomes visible to the VISAR when it overtakes the first shock. For the "2-ns square" pulses, the drive is off by the time the rarefaction reaches the ablation front.

Although the mechanism for generating this second shock is not the same as the second shock in a NIF capsule, there is no difference as far as what the VISAR measures (except perhaps preheat issues), or as far as what any particle of the liquid $\mathrm{D}_{2}$ feels.

For both drives, we used two types of targets, an $\mathrm{Al}$ (or polyimide) ablator with a thick layer of liquid $D_{2}$ or a sandwiched target consisting of an $\mathrm{Al}$ ablator-liquid $\mathrm{D}_{2}$-LiF window. For the latter targets, the VISAR detects the $D_{2}-\mathrm{LiF}$ interface velocity after the leading shock reaches the $\mathrm{LiF}$ when the LiF shock strength is less than $\sim 4 \mathrm{Mbar}$. When the resultant $\mathrm{LiF}$ shock strength is greater than $\sim 8 \mathrm{Mbar}$, the VISAR detects the shock velocity in the $\mathrm{LiF}$; when the LiF shock strength is between 4 to $8 \mathrm{Mbar}$, there is too little signal to detect in the VISAR image.

The figures below are initial comparisons of the shock velocity measured by the VISAR with LASNEX simulations for shots 29032311 (Figure 23) and 29040809 (Figure 24), respectively. Shot 29032311 is a " 2 -ns square" drive, which the simulation matches

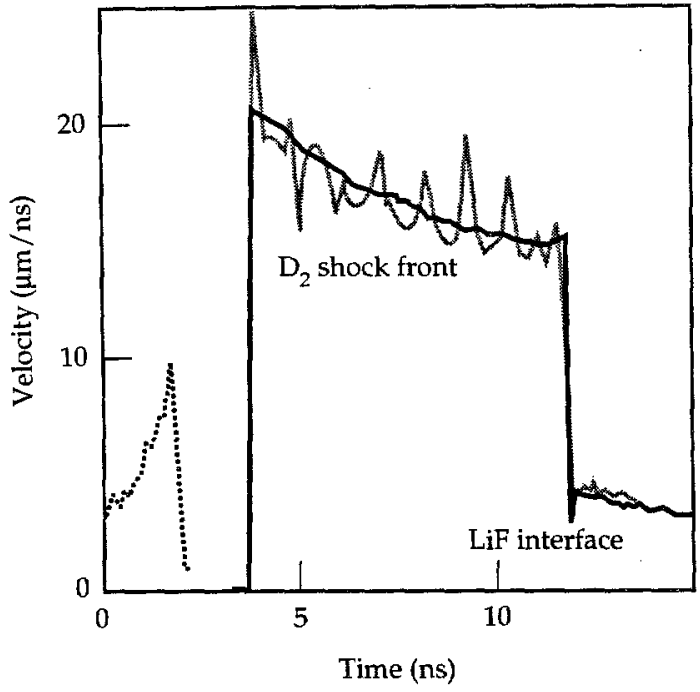

FIGURE 23. Shot 29032311 VISAR data (black) and simulation (gray). The laser power history is shown in dots. The simulation drive temperature was adjusted to match the observed initial shock strength, and the time axis was shifted $0.37 \mathrm{~ns}$ earlier to match the data. (08-00-0500-2767pb01)

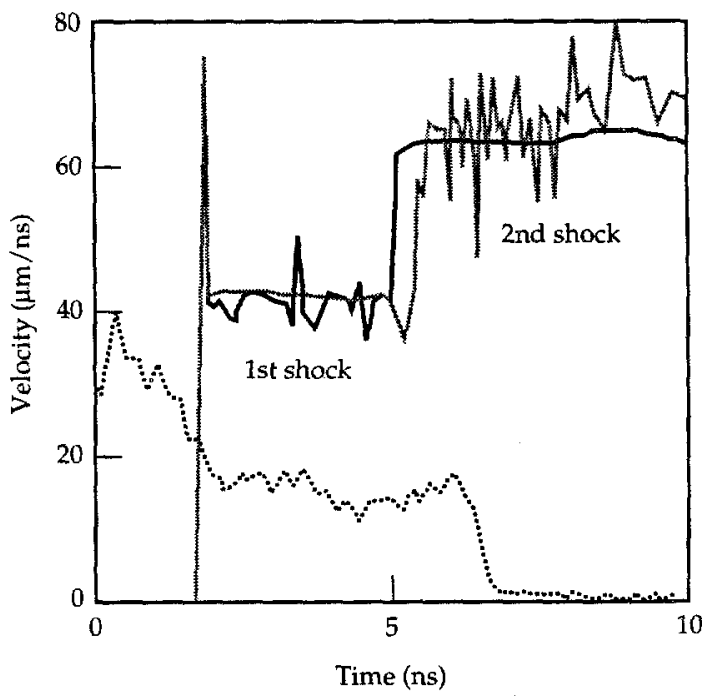

FigURE 24. Shot 29040809 VISAR data (black) and simulation (gray). The laser power history is shown in dots. The simulation drive temperature was adjusted to match the observed initial shock strength, and the time axis was shifted $0.6 \mathrm{~ns}$ earlier to match the data. (08-00-0500-2768pb01)

quite well; however, both the drive level and the timing have been adjusted to get this level of agreement. What the simulation does do is demonstrate that the decay rate of 
the shock velocity measured by the VISAR is perfectly plausible. Shot 29040809 requires even more adjustment, since the shock overtake time depends sensitively on the exact details of the radiation drive history. These and several similar experiment/simulation comparisons show the difficulty in predicting shock coalescence per drive, emphasizing the need for an engineering approach to shock timing on the NIF.

\section{Implosion Physics- Target Design}

Work in ignition implosion design this year was meant to increase our understanding in two ways: expanding the parameter space of possible ignition targets and deepening our understanding of the baseline targets.

We are broadening the parameter space of NIF ignition targets with continuing analysis on targets at drive temperatures other than the baseline $300 \mathrm{eV}$. Work at $350 \mathrm{eV}$, described in subsection "Integrated Calculations and Rayleigh-Taylor Analysis of 350-eV, 500-TW Be Target," has demonstrated distinct improvement in RayleighTaylor growth at the higher drive temperature. At the other extreme, at $250-\mathrm{eV}$ drive temperature, the Rayleigh-Taylor analysis suggests it will probably be necessary that these targets absorb more energy than the baseline at $300 \mathrm{eV}$. That is acceptable, since there is margin to increase the size of these targets as necessary. This work is described in subsection "Rayleigh-Taylor Analysis of $\mathrm{Be}$ and Polyimide Designs at 250-eV, 1.3-MJ Target."

Deepening our understanding of the baseline targets included several activities. We examined the impact of central gas density, reported in subsection "Sensitivity of Rayleigh-Taylor Growth to Central Gas Density." We are improving our 3D implosion capability with large solid-angle Rayleigh-Taylor simulations as described in subsection "3D Simulations with Large Solid Angle," and continuing to examine planned ignition diagnostics as reported in subsection "Simulations to Compare Compressed Core Diagnostic Techniques Required for Optimizing Ignition Targets."

\section{Integrated Calculations and Rayleigh-Taylor Analysis of 350-eV, 500-TW Be Target}

The 350-eV target is shown in Figure 25 with a diagram of the capsule in Figure 26. The drive is shown in Figure 27, showing the temperature vs time that drives the capsule and the corresponding laser power vs time. This target is close to being the largest that can be driven with NIF laser power, as it uses $600 \mathrm{TW}$. The pulse is short enough that this power level is probably acceptable. (Use of a low-albedo wall mixture could possibly increase the energy to the capsule by $10-20 \%$ or decrease the power requirement correspondingly.) This target performs well in integrated simulations, giving $70 \%$ of clean $1 \mathrm{D}$ yield. The integrated simulations required increasing case-to-capsule ratio from 2.5 to 3 (at waist) to avoid both hohlraum filling and asymmetric pressure

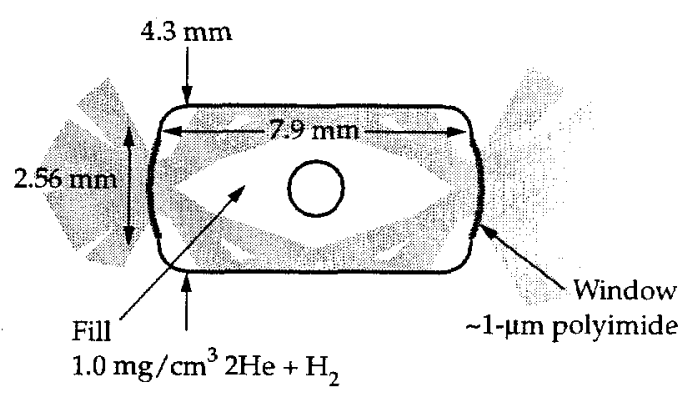

FIGURE 25. Target that could be driven by NIF with a peak hohlraum temperature of $350 \mathrm{eV}$. (50-00-0500-2751pb01)

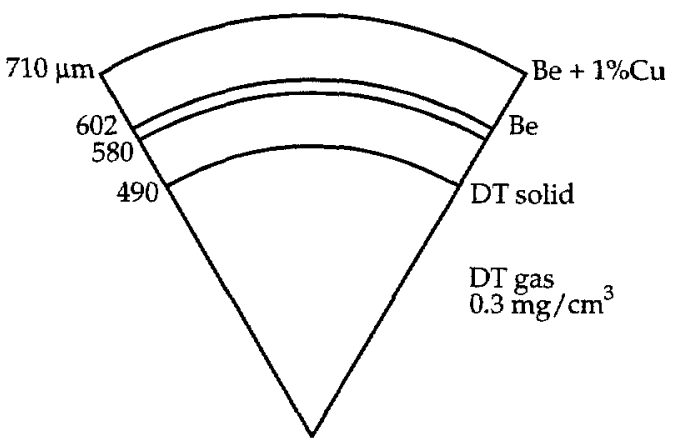

FIGURE 26. Pie diagram of capsule, 350-eV target. (50-00-0500-2752pb01) 


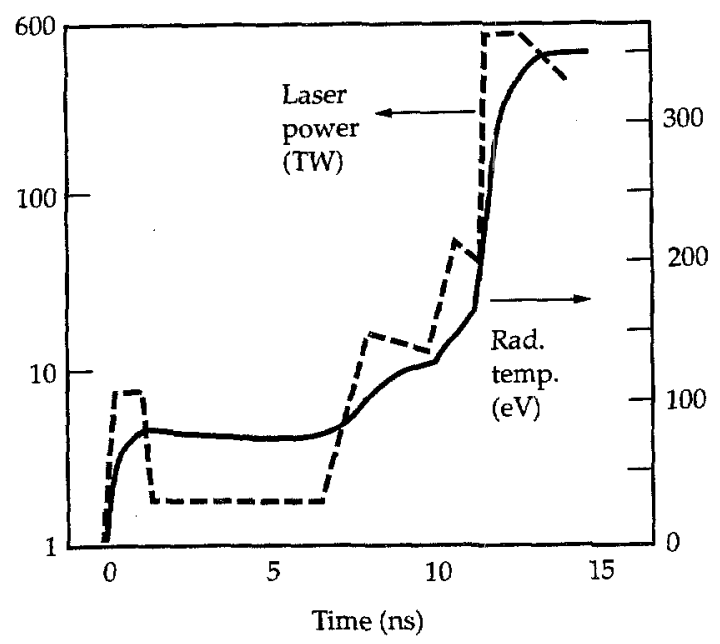

FIGURE 27. Temperature vs time that drives the 350-eV capsule and corresponding laser power vs time. (50-00-0500-2753pb01)

on the capsule from the hohlraum fill gas. At case-to-capsule ratio 2.5, various gas fills were considered; at low gas fills, the hohlraum filled unacceptably, while at higher gas fills a pressure spike on axis perturbed the capsule causing an asymmetric implosion even with the radiation on the capsule artificially symmetrized. For designs operating at $300 \mathrm{eV}$ or lower, there is an operating regime between these two failure modes, but at $350 \mathrm{eV}$ the case-tocapsule ratio has to be increased before a satisfactory gas fill can be found.

Rayleigh-Taylor analysis of this target, shown in Figure 28, shows very low growth. It can tolerate 5- $\mu \mathrm{m}$ DT $\mathrm{rms}$ and $>50$-nm ablator rms. For comparison, some results on other targets are also shown in Figure 28. The acceptable surface roughness specifications depend on the density of the central DT gas, as shown in Figure 29.

The target still accepts quite large perturbations, even with a high central gas density, although the specification is tighter.

\section{Rayleigh-Taylor Analysis of Be and Polyimide Designs at 250-eV, 1.3-MJ Target}

We performed perturbation growth analysis on two $250-\mathrm{eV}$ target designs, each using a drive profile that corresponds to $1.3 \mathrm{MJ}$ of laser energy and slightly less than 300 TW of power in a baseline gold hohlraum. One uses a beryllium ablator, doped with $0.9 \% \mathrm{Cu}$, inner and outer radii $1280 \mu \mathrm{m}$ and $1400 \mu \mathrm{m}$, and a fuel layer $80 \mu \mathrm{m}$ thick. This capsule absorbs $180 \mathrm{~kJ}$ and gives a yield of $26 \mathrm{MJ}$. The other uses a polyimide ablator, inner and outer radii $1340 \mu \mathrm{m}$ and $1425 \mu \mathrm{m}$, with an inner mandrel of $\mathrm{CH}+2 \% \mathrm{Ge}, 25 \mu \mathrm{m}$ thick, and an 85$\mu \mathrm{m}$-thick fuel layer. This capsule absorbs $160 \mathrm{~kJ}$ and gives a yield of $17.3 \mathrm{MJ}$. The absorbed energy is lower, for a very similar drive profile, because the polyimide
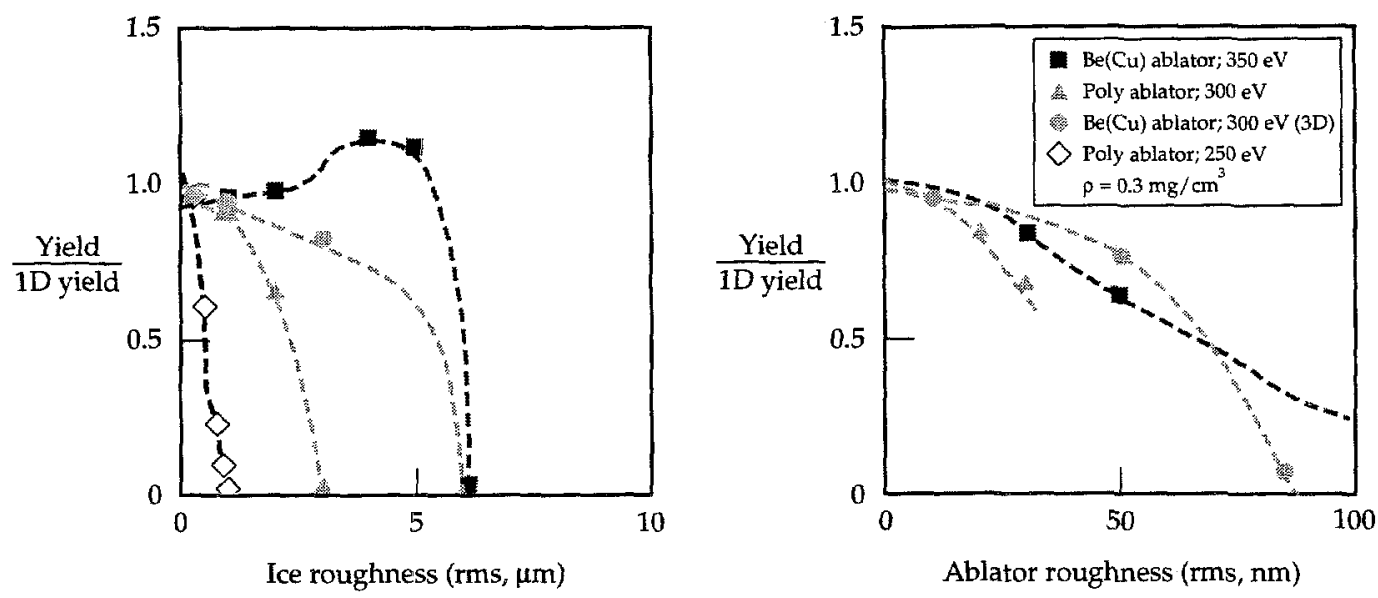

FIGURE 28. Yield vs surface roughness for 350-eV target, showing a comparison with other targets at $300-\mathrm{eV}$ and $250-\mathrm{eV}$ peak drive temperature. The sensitivity to surface perturbations is a strong function of drive temperature.

(50-00-0500-2754pb01) 

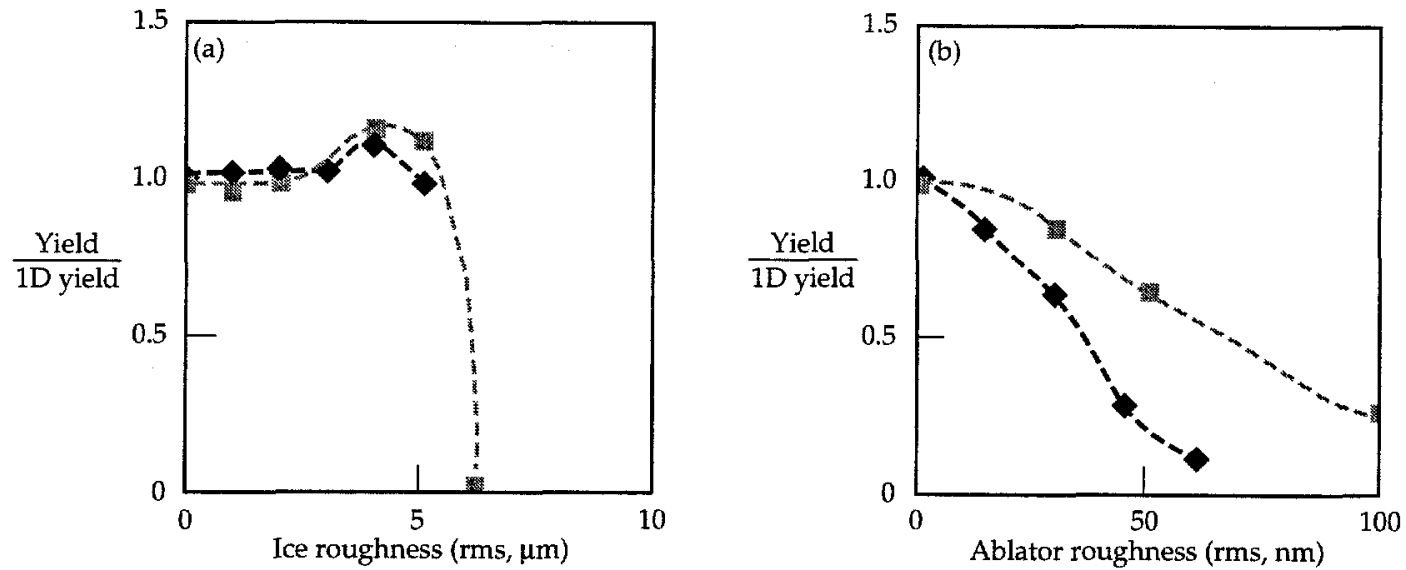

FIGURE 29. Yield vs surface roughness for a 350-eV target, showing the sensitivity to central DT gas fill. (a) Yield vs ice surface roughness. (b) Yield vs ablator roughness. In both plots the diamonds show the result with central gas density of $0.6 \mathrm{mg} / \mathrm{cm}^{3}$, and the squares show the more robust performance with the central gas density $0.3 \mathrm{mg} / \mathrm{cm}^{3}$. (50-00-0500-2755pb01)

has a higher albedo. Both capsules have $0.3 \mathrm{mg} / \mathrm{cm}^{3}$ central DT gas fill.

These targets are very susceptible to Rayleigh-Taylor instabilities. This result is about what one might expect, given their position in power/energy space (shown in Figure 30). The low-power boundary of the ignition regime is determined by the Rayleigh-Taylor specification, and these targets are just outside the regime where

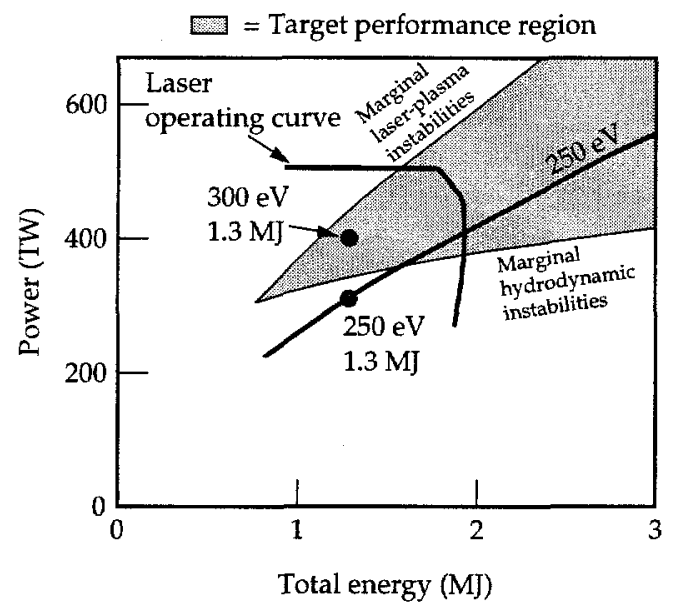

FIGURE 30. The parameter space for ignition targets, showing the central design point at which most work has been done, the 300-eV 1.3-MJ targets, in the center of the $\mathrm{NIF}$ operating regime, and the 1.3-MJ 250-eV target, which is very marginal with respect to hydrodynamic instabilities. (50-00-0500-2756pb01) specifications are expected to be acceptable. The detailed Rayleigh-Taylor simulations shown in Figure 31 confirm that perturbations must be very small for these targets to perform. Several recourses are available if laser-plasma instabilities or other unexpected issues force us to use targets driven at only $250 \mathrm{eV}$. First, the capsules can be made larger than was assumed for this work, in which we stayed at $1.3 \mathrm{M}$ J to compare with the 1.3-MJ work at $300 \mathrm{eV}$. Also, it is likely that there is room to optimize the target better at $250 \mathrm{eV}$, especially reconfiguring the hohlraum to get more energy into the capsule.

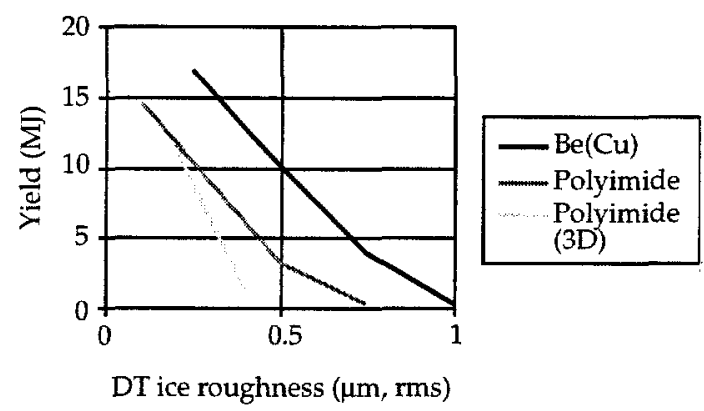

FIGURE 31. Sensitivity of 1.3-MJ 250-eV target to perturbations initially on the DT ice surface. The polyimide target can only tolerate perturbations smaller than $0.5 \mu \mathrm{m}$ rms. The beryllium target is somewhat less sensitive. (50-00-0500-2757pb01) 


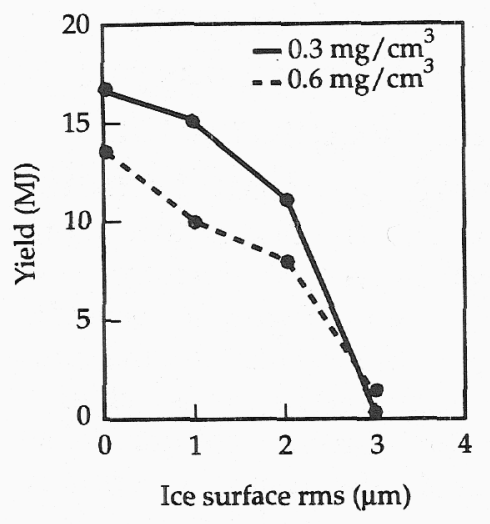

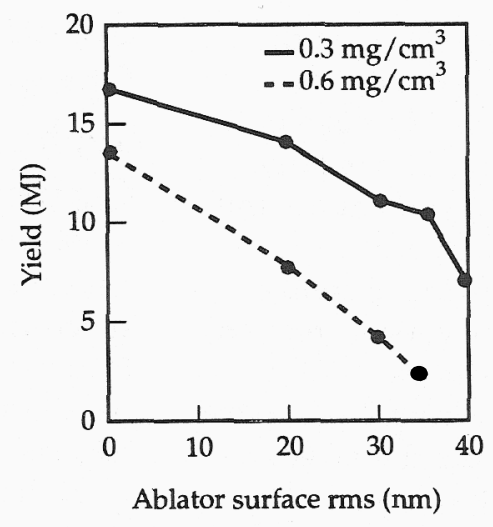

Figure 32. Yield vs surface roughness for a baseline 300-eV polyimide target, showing the sensitivity to the central DT gas density. Achieving adequate DT ice roughness may require working at a higher temperature and a correspondingly higher DT gas density; this tightens the roughness specifications, but not unacceptably.

(50-00-0500-2758pb01)

\section{Sensitivity of Rayleigh-Taylor Growth to Central Gas Density}

The central gas density is determined by the operating temperature of the cryogenic capsule. Originally it was optimized at $0.3 \mathrm{mg} / \mathrm{cm}^{3}$, which corresponds to a temperature $1.5 \mathrm{~K}$ below the triple point temperature of $19.8 \mathrm{~K}$. Recent work on layer characterization indicates that the quality of the cryo layer depends quite strongly on the temperature, being smoother at temperatures close to the triple point, while the target performance has a somewhat weaker contrary dependence. Ultimately the temperature and gas density will have to be set to minimize the net effect on the capsule performance, as a decrease in temperature increases the initial roughness while decreasing the sensitivity to Rayleigh-Taylor growth. The sensitivity of the baseline $300-\mathrm{eV}$ polyimide design is shown in Figure 32. While the performance suffers somewhat at higher gas fill, and the ablator surface specification is somewhat tighter, it appears that there is an option of operating at the triple-point density of $0.6 \mathrm{mg} / \mathrm{cm}^{3}$. Similar sensitivity is seen in the $350-\mathrm{eV}$ target as shown above in Figure 29.

\section{D Simulations with Large Solid Angle}

An important goal in ignition target design is to perform 3D simulations that include both asymmetry and mix, with enough solid angle to see most relevant modes. During this year we began work on the best possible estimate of a nominal 3D asymmetry. In addition, simulations with Rayleigh-Taylor only were pursued without an estimated asymmetry. These simulations were performed with sufficient solid angle $\left(72^{\circ} \times 72^{\circ}\right)$ to ensure that all low-mode RayleighTaylor effects are modeled. The simulation was done on a standard $300-\mathrm{eV}$ $\mathrm{Be}(\mathrm{Cu})$ design. It predicts good yield, 15.4 MJ with nominal initial surface roughness: $1 \mu \mathrm{m}$ on the ice and $20 \mathrm{~nm}$ on the ablator. Figure 33 shows a density isocontour at a time slightly before ignition; the density of $13.8 \mathrm{~g} / \mathrm{cm}^{3}$ was chosen since it best defines the shell.

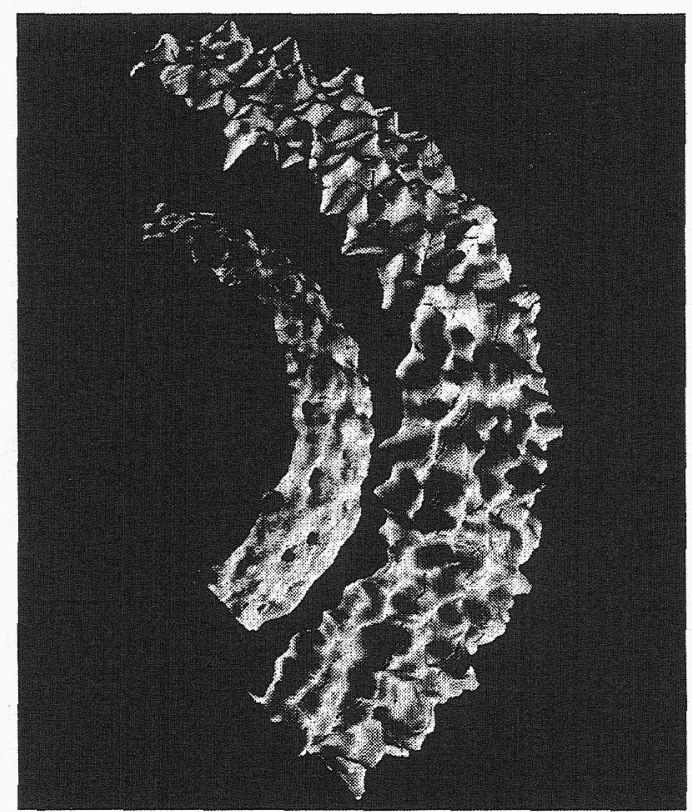

FiguRE 33. Density isocontour surfaces in a large 3D simulation of Rayleigh-Taylor growth on a $\mathrm{Be}(\mathrm{Cu}) 300-\mathrm{eV}$ capsule. The density of $13.8 \mathrm{~g} / \mathrm{cm}^{3}$ was chosen since it best shows the two sides of the shell at a time slightly before ignition.

(50-00-0500-2759pb01) 
Perturbations that have grown on the outer surface of the shell during the acceleration phase are clearly visible. It is obvious that the simulation is large enough in solid angle to encompass the largest growing modes. There are enough zones to resolve the growing modes (320 in each transverse direction). Figure 34 shows a density isocontour defining the shell as it burns, in this case at $609 \mathrm{~g} / \mathrm{cm}^{3}$. At this time the target has produced $1 \mathrm{MJ}$ of yield. Bubbles of lowdensity fuel have risen through the shell as it ignites, and crater-like features are evident where beryllium fingers are penetrating the isocontour, which is otherwise in DT fuel. Again, it is evident that the simulation has enough solid angle to encompass the relevant modes, and that the relevant modes are resolved.

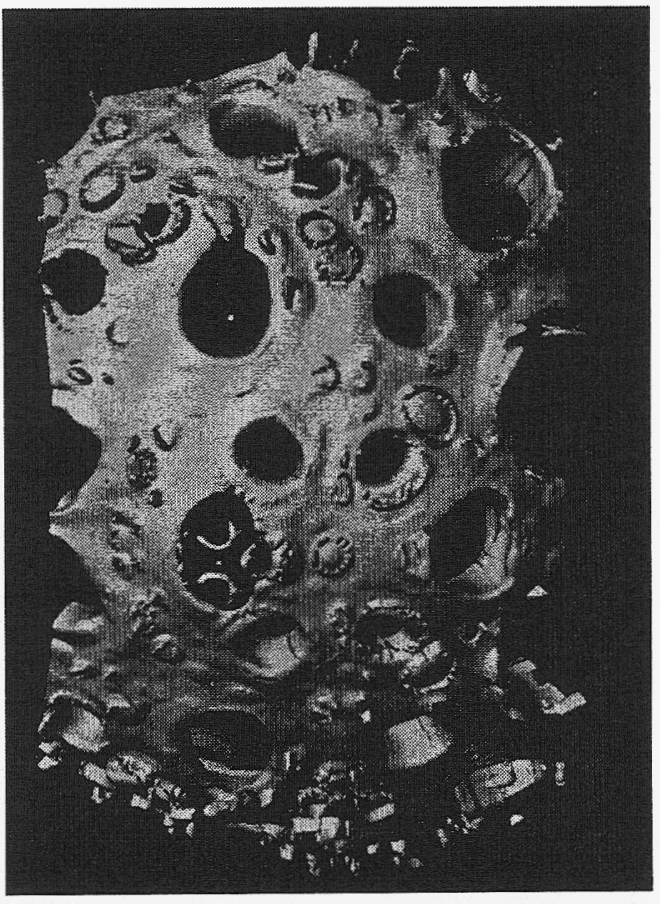

FIGURE 34. Density isocontours slightly after ignition in a large 3D Rayleigh-Taylor simulation. At this time the capsule has produced $1 \mathrm{MJ}$ of yield and is burning robustly. The density of $609 \mathrm{~g} / \mathrm{cm}^{3}$ shows the high-density part of the fuel. The holes are where bubbles of hotspot material have risen through the shell. The crater-like features are where spikes of beryllium have touched the high-density DT shell. (50-00-0500-2760pb01)
Simulations to Compare Compressed Core Diagnostic Techniques Required for Optimizing Ignition Targets

Work this year has concentrated on the neutron spectrum. A typical neutron spectrum is shown in Figure 35. Several features of possible interest are: the number of down-scattered neutrons, which depends on the burn-time column density of the capsule; the number of primary neutrons, as a measure of the yield; the width of the primary neutron peak, which depends on the temperature of the burning fuel; and the high-energy neutrons, which are made by up-scattering processes, primarily the tertiary process in which a primary neutron scatters up an ambient deuteron or triton that in turn reacts in-flight to produce a high-energy neutron. For low column densities, in which none of the in-flight particles are slowed significantly, the relative number of tertiary neutrons is proportional to the column density squared. Figure 36 shows the fraction of tertiary neutrons for a wide variety of implosions, plotted vs column density squared. It is evident that for some cases, such as the scaled implosions that could possibly be done

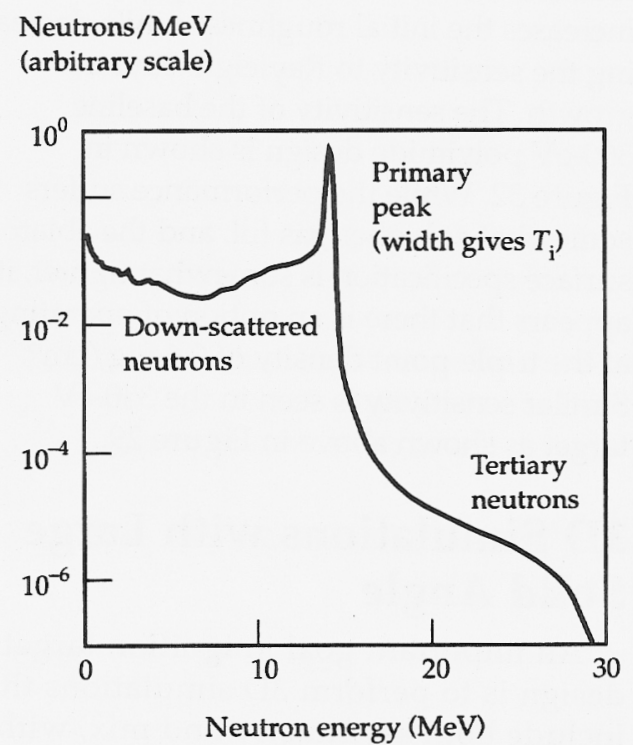

FIGURE 35. The neutron spectrum produced by a typical NIF ignition capsule. (50-00-0500-2761 pb01) 


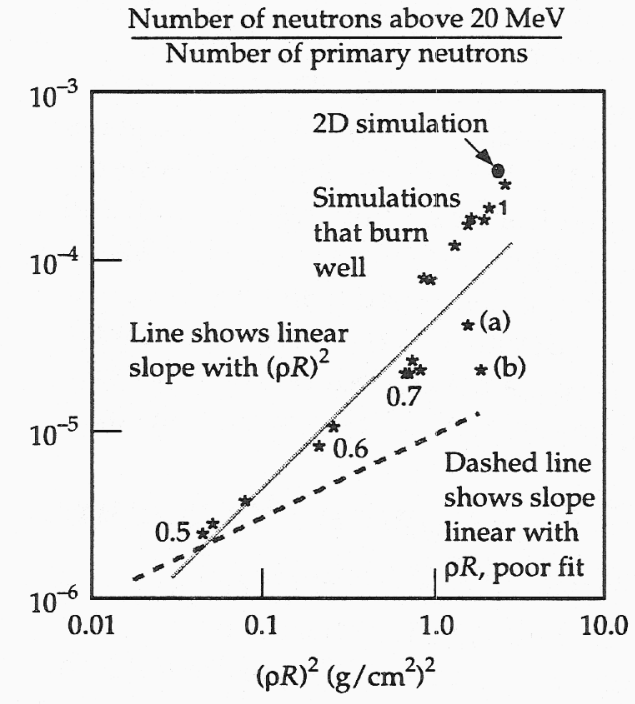

FIGURE 36. The fraction of neutrons produced at high energy, plotted against column density squared. In the limit where none of the particles are stopped (that is, low column density), the tertiary signal is proportional to column density squared. Points with numbers are direct scales of the full-scale target, with the scale as indicated. (All dimensions and times are scaled by this factor, producing a hydrodynamically equivalent implosion.) Simulations that burn well are indicated and give higher tertiary signals. Points (a) and (b) are high column density failures; (a) is a scale 1.0 target with drive flux reduced by $50 \%$ and $(b)$ is a scale 2.0 target with drive flux reduced to $20 \%$ of the scaled value. (50-00-0500-2762pb01)

with less than 192 beams, the tertiary signal will be a direct measure of column density. For the higher column densities interpretation will be more complex.

\section{Implosion Physics- Advanced Diagnostics Development}

In FY99, we began a concerted effort to identify the diagnostics instrumentation required for the ignition campaign on the NIF. The required measurements and specifications were generally derived from an examination of various capsule failure modes using LASNEX calculations. A reasonably complete list of ignition failure signatures was developed based on these calculations. In many cases, these signatures can be measured or extracted from well-established diagnostic techniques (e.g., $\mathrm{Cu}$ activation and the thermal broadening of the primary neutron line emission). However, in other cases, the measurements will require the development of entirely new techniques. In particular, distinguishing hot-spot phenomena from burning-fuel emission will require spatially and/or temporally resolved measurements, which are impractical at the much lower yields available at OMEGA or Nova. Measurements such as the spatially resolved hot-spot and burning-fuel ion temperature and the temporal evolution of the hot-spot ion temperature require significant yields and sophisticated new diagnostic instrumentation. Finally, there are cases in which altogether new probes will be utilized at the NIF. High-energy charged particles (both primary fusion reaction products as well as knock-on fuel ions) will be used to probe the angular dependence of the fuel $\langle\rho R>$ while the primary DT fusion $\gamma$-ray $(11-16 \mathrm{MeV})$ will be used to measure the fusion reaction history and bang time.

The research and development for these new diagnostic techniques has been prioritized according to the current diagnostic requirements of the ignition campaign (for example, many of the advanced ignition diagnostics will not be required until after hohlraum tuning is complete). Diagnostics research and development is currently being conducted on both $x$-ray-based instrumentation as well as on new concepts for charged particle spectroscopy, neutron spectroscopy, neutron imaging, and $\gamma$-ray spectroscopy. In many cases, this work is being conducted in collaboration with scientists from the other national laboratories (Los Alamos and the Laboratory for Laser Energetics) as well as with scientists from various universities (in particular, Massachusetts Institute of Technology (MIT) and State University of New York at Geneseo). These collaborations have significantly enhanced the effective LLNL advanced diagnostics R\&D effort. The research highlights for FY99 include the activation of a second charged particle spectrometer at the OMEGA laser, the study of the $12 C(n, 2 n) 11 C$ reaction for tertiary neutron spectroscopy, and the preliminary design of a radiochemical diagnostic 
based on gas sampling and mass spectroscopy. The possibility of using highenergy $x$-rays $(>10 \mathrm{keV})$ to image the burning-fuel core was tested at OMEGA.

\section{An Electronic Detector for the LLNL-MIT Charged Particle Spectrometer at OMEGA}

The two LLNL-MIT charged-particle spectrometers (CPS) are currently read out using a nuclear emulsion (CR-39) technique. While the quality of the data is superb, the process required to etch and scan the CR-39 for charged-particle tracks is both laborious and time-consuming. Therefore, it is impractical for the CPS data to be used to guide or establish shot conditions during a particular campaign. An electronic readout of the primary charged-particle lines (primary and secondary protons from the DD reaction, alpha particles from the DT reaction, and primary protons from the $D^{3} \mathrm{He}$ reaction) could provide online measurements of these yields and yield ratios within minutes of a shot.

During FY99, the specifications for such a readout system were developed, and an initial test of a silicon-based detector was carried out using the CPS1 at OMEGA. Two silicon surface barrier detectors were placed in the bend plane of the CPS1 magnet and the signals recorded on a fast digital oscilloscope. The measured signals indicated that there was a significant capacitive coupling between the silicon detectors and the CPS housing that was attached to the target chamber and, consequently, poorly grounded. Furthermore, the bias voltage used on the silicon detectors was also poorly isolated from the chamber ground, which resulted in a further capacitive ringing that overwhelmed any charged-particle signal. After analyzing the data it was decided that the next test, scheduled for FY00, would utilize much lower capacitance PIN (positiveintrinsic-negative) diodes with a much better designed test setup to minimize the capacitive coupling with the target chamber. Furthermore, a $100-\mathrm{V}$ battery would be used to provide a noise-free bias to the PIN diodes. These tests should establish the proof-of-principle for a silicon-strip-based electronic readout system for the CPSs.

Figure 37 shows the PIN diode test assembly that has been designed for shots on CPS1 in FY00. These PIN diodes were acquired from the LLNL test program and modified for the CPS test assembly. The test assembly is composed primarily of nonconductive delrin, which should significantly decrease the noise produced by capacitive coupling with the CPS vacuum housing.

\section{NIF Full-Aperture Backscatter Station (FABS) Design}

The Full-Aperture Backscatter Diagnostic (FABS) will measure the light scattered back into the laser optics by the target. When combined with data from the Near Backscatter Imager (NBI), the FABS will allow the coupling efficiency of the laser and target to be determined. The FABS will look through the final turning mirror (LM8) of twenty different beams located in five different quads and provide the time and spectral response of the scattered light collected over the nearly entire beam aperture as well as a lens plane image of the light. The FABS design was developed during the year and modified to accommodate changes in the final optics assembly (FOA) target area mirrors and structures, and in particular, the wedged lens. An optical collection system has been

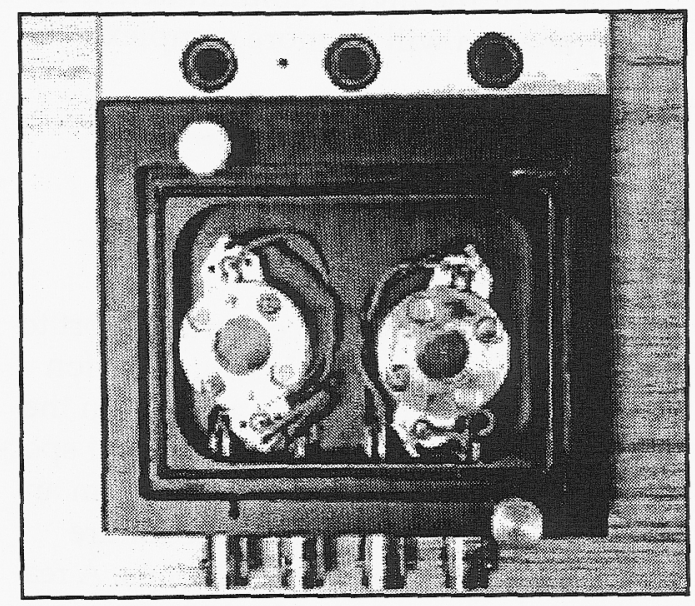

FIGURE 37. PIN diode test assembly for shots on CPS1 at OMEGA. (40-00-1200-6347pb01) 
designed that will collect the light across the full beam aperture and over the necessary wavelength ranges (700 to $400 \mathrm{~nm}$ and 354 to $348 \mathrm{~nm}$ ) in the presence of the wedged final focus lens as shown schematically in Figure 38. This system provides the necessary spot quality and insensitivity to wavelength to couple a full-aperture sample of the light into a streaked spectrometer with the required sensitivity and spectral and temporal responses. A complete mechanical design was carried out for the mirror support structure that will allow $\geq 85 \%$ of the beam aperture to be viewed while maintaining sufficient mirror flatness that the incident beam quality is not affected. This design shares common mirror mount points and mounting and actuator hardware so that the installation, operation, and maintenance procedures for the transmissive LM8 (LM8T) will be very similar to the standard LM8. The design also accommodates the beam and interface properties at all 20 FABS locations and so is interchangeable. Laboratory tests of the effect of UV light on optical materials were carried out and allowed the LM8T mirror material to be identified as $\mathrm{Bk} 7$ glass. The FABS design is now being updated with the most recent modifications to the mirror design and target area. A formal Conceptual Design Review (CDR) was held for the Joint Central Diagnostics Team review committee, which reviewed all of the FABS design progress since the original CDR, and all concerns about the instrument were addressed.

\section{Calibrating Dante XRDs in Preparation for Cross- Calibration of Dante with DEMIXART from CEA on OMEGA}

The $x$-ray diodes (XRDs) from the Nova Dante soft $x$-ray power diagnostic were shipped to the LURE (Laboratoire Utilize de Radiation Electromagnetique) synchrotron in France. There, they were calibrated using the same beamlines to calibrate the XRDs that the French used in

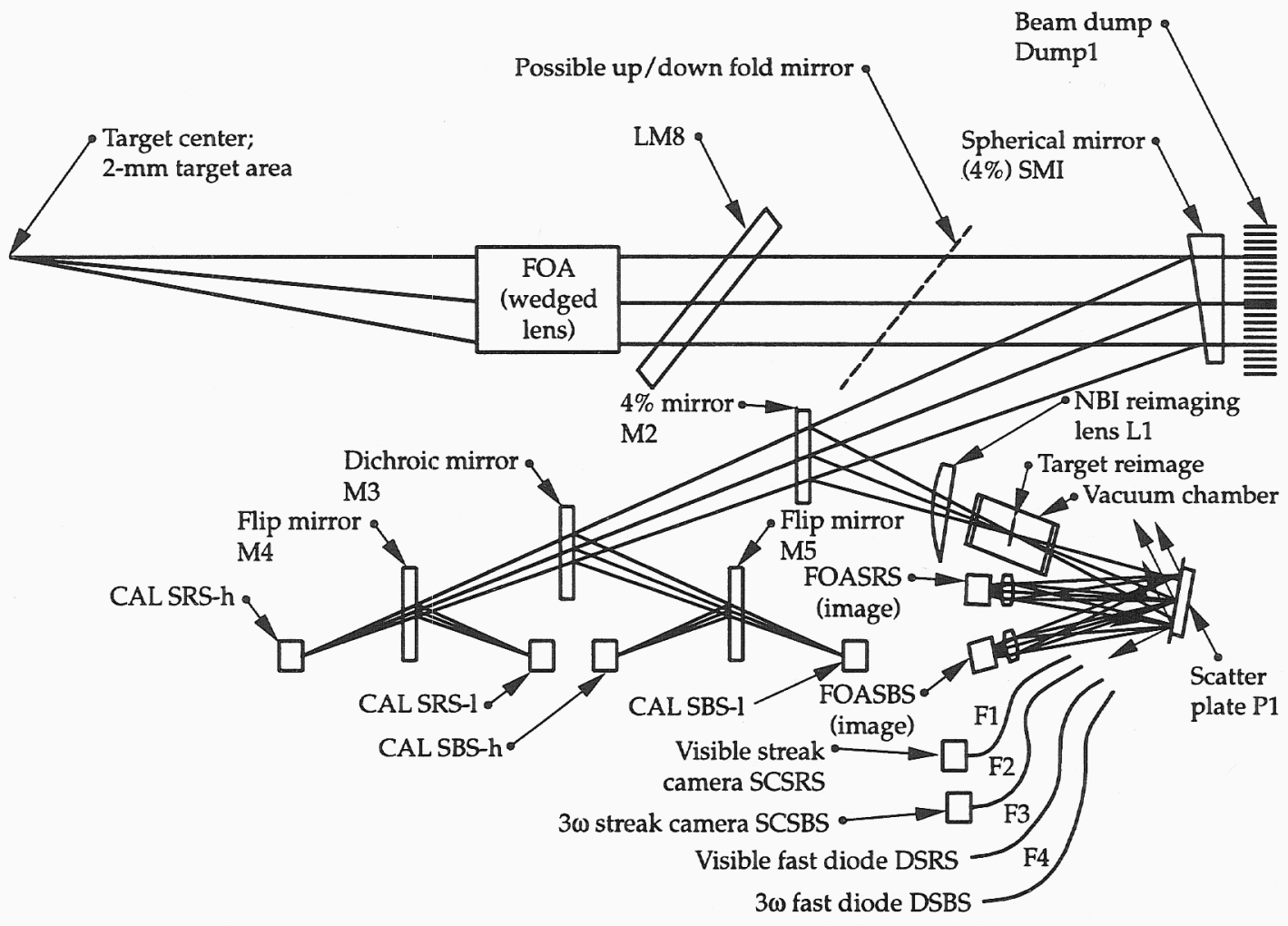

FIGURE 38. Schematic of the FABS optical collection system and the integrated incident beam optical components, showing wedged lens and transmissive LM8 mirror. (Drawing not to scale.) (40-00-1200-6348pb01) 
their soft $x$-ray diagnostic, DMX. This was done to eliminate the usual major uncertainty in comparing the Dante and the DMX, namely, the uncertainty in the calibration source.

The calibration of the Dante XRDs was straightforward, as their photocathodes are large compared to the spot size coming out of the synchrotron monochrometer. Likewise, the silicon diode used as a reference standard is large compared to the spot size. The DMX photocathodes, on the other hand, are small compared to the spot size. To calibrate them, the silicon reference diode had a slit placed in front of it. The reference was then scanned across the beam, to insure that it was uniform over the 1-mm size of the DMX photocathode. The DMX calibrations rely on this uniformity, as the sensitivity is spatially dependent, being more sensitive at the edge of the cylindrical detector.

The LURE calibrations (labeled CEA in Figure 39) seem to show the Dante XRDs as less sensitive than measured at Brookhaven National Laboratory (BNL). This may be due to a systematic error in the calibration process that has gone unnoticed. All detectors with similar photocathode material $(\mathrm{Al}, \mathrm{Ni}$, or $\mathrm{Cr})$ showed similar sensitivity, suggesting that the materials have all come to equilibrium with the environment: similar amounts of carbon and oxygen contaminants.

The XRDs have all been installed on the Dante at OMEGA.

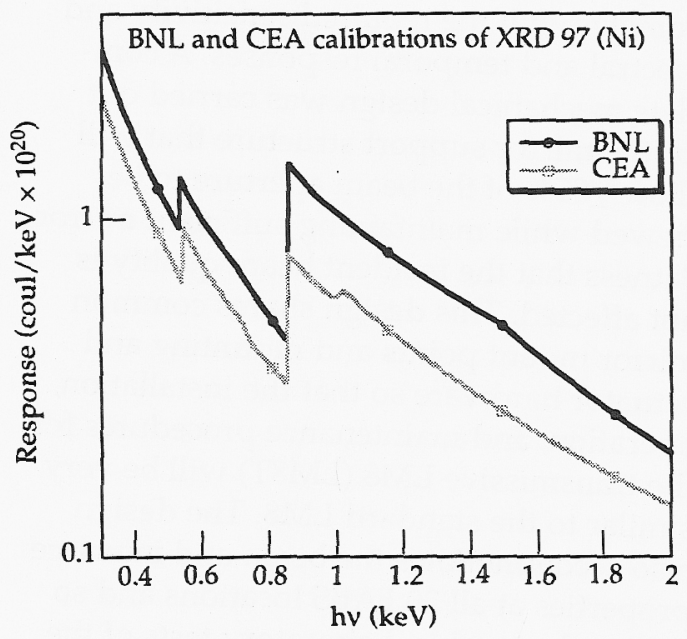

FIGURE 39. The French Atomic Energy Commission (CEA) calibrations of the Dante $x$-ray diodes compared with those of Brookhaven National Laboratory (BNL). 40-00-1200-6349pb01) 


\subsection{High ENERGy Density EXPERIMENTAL SCIENCE}

$\mathrm{T}$ he High Energy Density Experimental Science (HEDES) effort within the Laser Programs Directorate consists of laser experiment projects in the areas of hydrodynamics, radiation flow, and implosions, conducted on the Nova and OMEGA lasers. This work is done in support of, and in collaboration with, the Defense and Nuclear Technologies Directorate and (for the x-ray source development in the radiation flow project) the Defense Threat Reduction Agency. In addition, HEDES collaborated in an equation-of-state (EOS) effort with the Physical Database Research Program.

During the past year, all experimental activities on Nova were transferred to OMEGA at the University of Rochester in preparation for the closure of Nova at the end of May. Several experimental activities were concluded on Nova in the first part of the year: material strength experiments, double-shocked hydrodynamics, ablatively driven linear Rayleigh-Taylor (RT) growth, and doubly shocked $\mathrm{D}_{2}$ EOS experiments. Subsequently, new experimental capabilities were evaluated and developed on OMEGA to explore some of its unique capabilities in preparation for future experiments: direct drive (for planar hydrodynamics) and radiation flow. A new diagnostic was implemented that significantly improved the quality of the data taken for the measurement of hydrodynamic features.

\section{Experiments Measuring Material Strength Effects on Instability Growth and Melt Characterization}

\section{Instability Growth}

An RT instability growth experiment was performed on Nova to study the effect of material strength on the growth of a preimposed modulation at an RT unstable interface. In this experiment, a 20- $\mu \mathrm{m}$-thick Al foil was used. A 1.0- $\mu$ m peak-valley sinusoidal modulation was machined on the surface of the $\mathrm{Al}$ foil, and then this foil was thermally pressed with a $20-\mu m$-thick $\mathrm{CH}(\mathrm{Br})$ ablator. The machined perturbation was embedded at the ablator-Al interface (Figure 1).

The goal of this experiment was to compress and accelerate the thin foil using a sequence of two shocks to keep the foil solid under compression. The foil package was mounted on the side of an internally shielded hohlraum. The internal shielding in this hohlraum was designed to prevent preheating of the foil sample by blocking the M-band x-ray direct line of sight from the laser beam spots to the package.

An 8.5-ns laser pulse was generated by temporally staggering eight of the Nova beams. A 4-ns low-power foot pulse provided a first shock with pressure in the 
(a) Hohlraum target

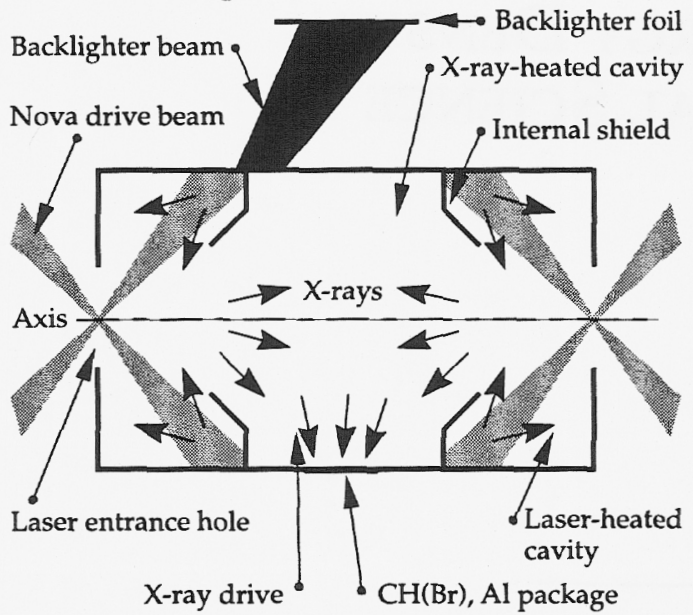

(b) Rayleigh-Taylor package

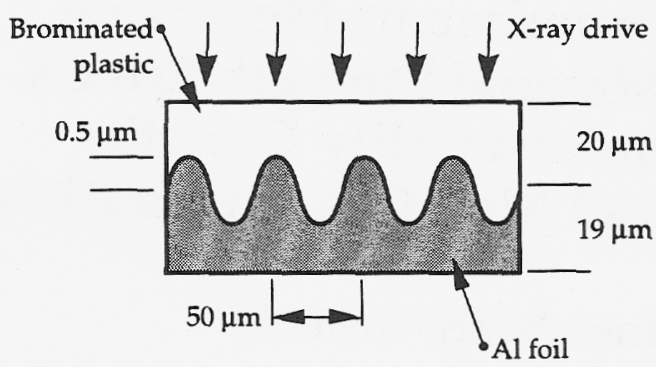

FIGURE 1. Schematic of the experimental setup to study the effect of material strength on the growth of a preimposed modulation at an RT unstable interface. (40-00-1100-6096pb01)

$\mathrm{Al}$ of about 200-300 kbar. The pulse then ramped up to launch a second shock with peak pressure of $1.8 \mathrm{Mbar}$ in the Al. The laser power was held constant from 5.5 to $8.5 \mathrm{~ns}$ to provide a period of acceleration for instability growth.

The instability growth was measured for sinusoidal perturbations with wavelengths of 10,20 , and $50 \mu \mathrm{m}$. In the case of the shorter wavelengths, the data shows nearly fluid-like growth followed by stagnation of the growth. A plot of the measured growth factors as a function of time is shown in Figure 2 for the 20- $\mu \mathrm{m}$ wavelength perturbation. The data is plotted from a total of seven separate Nova shots. In each case, the growth appears to be nearly fluid-like at early times, but reduced compared to fluid at later times.

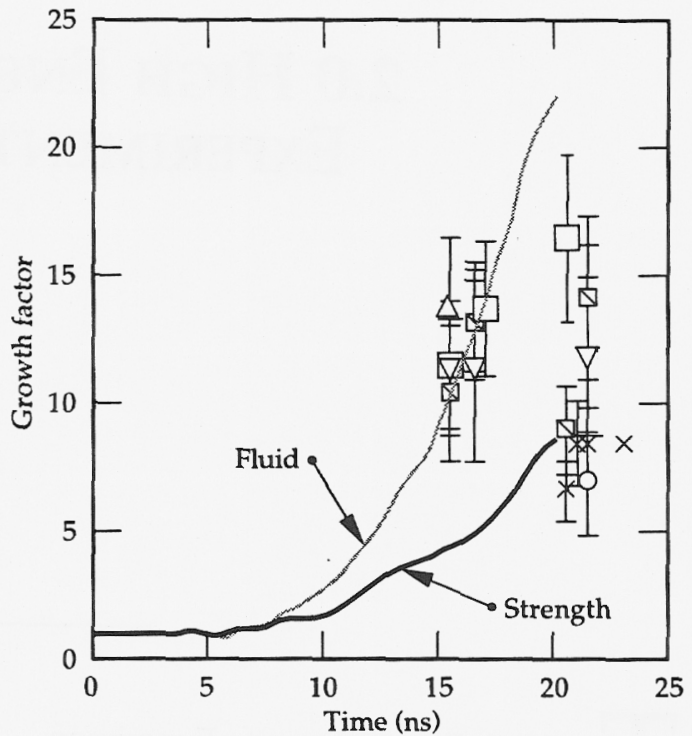

FIGURE 2. A plot of the measured growth factors as a function of time for the 20- $\mu \mathrm{m}$ wavelenght perturbation. Growth appears to be nearly fluid-like at early times, but reduced compared to fluid at later times. (40-00-1100-6097pb01)

This result is suggestive of the model of localized heating in shear bands by Grady and Asay. ${ }^{1}$ With this picture, the $\mathrm{Al}$ responds to the shear stresses from the shock passage by dissipating the elastic energy in localized shear bands. These narrow bands are heated and they melt, resulting in bulk flow as if the foil were fluid. The heat in the shear bands conducts into the bulk material, and the foil recovers an enhanced strength, perhaps due to a high dislocation density resulting from the initial shocks.

The instability growth experiments with Al were conducted on Nova during fiscal year 1999 (FY99). This includes side-on foil trajectories for drive characterization and RT growth experiments. Modeling of the hohlraum drive and instability growth experiments was also done during this period.

\section{Melt Characterization}

The technique of dynamic $x$-ray diffraction is being developed to demonstrate that the foil samples used in the RT instability experiments remain solid. During FY99, only a few shots were dedicated to 
this effort. For these experiments, macroscopic samples of single crystal Al were mounted on the side of an internally shielded hohlraum. These crystals were shocked at low pressure to simulate the first shock in the Al. The results were inconclusive. In order to focus on the RT experiments, we deferred these experiments to OMEGA.

This work is being done on OMEGA in conjunction with scientists from the University of Oxford, the University of California at San Diego (UCSD), Los Alamos National Laboratory (LANL), and the University of Rochester's Laboratory for Laser Energetics (LLE) under a National Laser Users' Facility (NLUF) project. During FY99 there were three days of shots devoted to transitioning the diffraction experiments to OMEGA, where they are done in a directdrive configuration. Simultaneous diffraction from orthogonal lattice planes of $\mathrm{Si}$ and $\mathrm{Cu}$ were recorded. A sample of the timeintegrated diffraction data is shown in Figure 3. This shows diffraction from compressed $\mathrm{Cu}$ in two orthogonal directions.

\section{Evaluating OMEGA for Direct-Drive Experiments Studying Planar Hydrodynamics}

With the transition to OMEGA, it was important to reassess the testbed we use to drive hydrodynamic experiments for three reasons:
1. To determine if OMEGA could offer specific advantages for those experiments. Unlike Nova, the illumination geometry on OMEGA is optimized for direct drive. It was important to determine whether direct drive or indirect drive was the optimal technique prior to conducting a series of hydrodynamic experiments.

2. To determine whether direct or indirect drive was optimal for NIF. This assessment is required to understand the trade-offs in choosing a start-up sequence for NIF. Incidence angle of beams is one factor that must be evaluated to settle on a start-up sequence.

3. To engineer a testbed that was both optimized and well characterized. For Richtmyer-Meshkov instabilities, that requires an interface that is constant velocity, has maximum travel distance, and has acceptable planarity across the measurement region, while avoiding such effects as hohlraum filling for indirect drive.

The 1D performance of laser or $x$-ray driven targets to study phenomena such as the Richtmyer-Meshkov instability in a single, steady-shock, step-down-in-density system was described by a simple model based on 1D hydrodynamics. The distance the interface travels under constant-velocity conditions is a multiple of the separation between the ablation and shock front, and this multiple depends on the density ratio

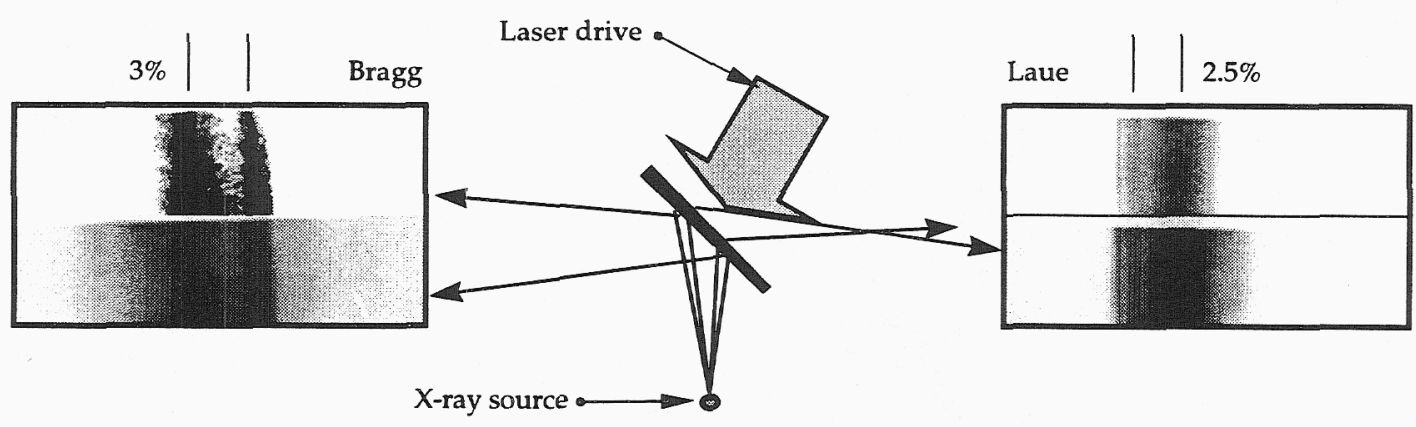

FIGURE 3. A sample of the time-integrated diffraction data from compressed $\mathrm{Cu}$ in two orthogonal directions. (40-00-1100-6098pb01) 
at the interface and the equations of state of the two materials. The model is applied to NIF and OMEGA with the aid of 1D hydrocode simulations to predict the ablation-shock separation. If adequate interface planarity can be maintained over an experimental length equal to the focal spot diameter, direct drive may significantly outperform indirect drive at the same pulse length and typically $\geq 2$ at the same ablation pressure. The results are shown in Figure 4 for direct drive and for indirect drive. Plots are optimized target configurations where the drive is maintained until interface rarefaction reaches the ablation front. The drive for NIF is assumed to be 13-ns-long, staggered pulses so that the optimum conversion efficiency to $3 \omega$ is maintained. It can be seen that with the same number of beams on NIF, direct drive outperforms indirect drive, even running at half energy with direct drive.

Over the next year, we will be testing these predictions on OMEGA through scaled experiments. The major uncertainties are the ability to control 2D effects in the directly driven targets (critically) and the optimum hohlraum performance due to hohlraum filling in indirect drive. We can perform a $1 / 7^{\text {th }}$-scale experiment on OMEGA with direct drive to test laser coupling and 2D hydrodynamic effects. Experiments have started on OMEGA to measure the effect of beam angle on interface travel and planarity.

\section{Target Geometry and Initial Two-Shock Experiments on Nova}

We have measured the growth of hydrodynamic instabilities in a doubly shocked experiment. Previous shock-tube experiments on double-shocked mixing layers indicate that the growth rate of the mixing region is greatly enhanced upon passage of the second shock, showing a rapid transition to turbulence. In these Nova experiments we can achieve higher Mach numbers than shock tubes. A halfhohlraum driver was used to launch a shock into a miniature shock tube that then crossed a rippled interface, causing
FIGURE 4. Plots comparing direct drive and indirect drive on NIF and OMEGA with the aid of $1 D$ hydrocode simulations to predict the ablation-shock separation.

(40-00-1100-6099pb01)

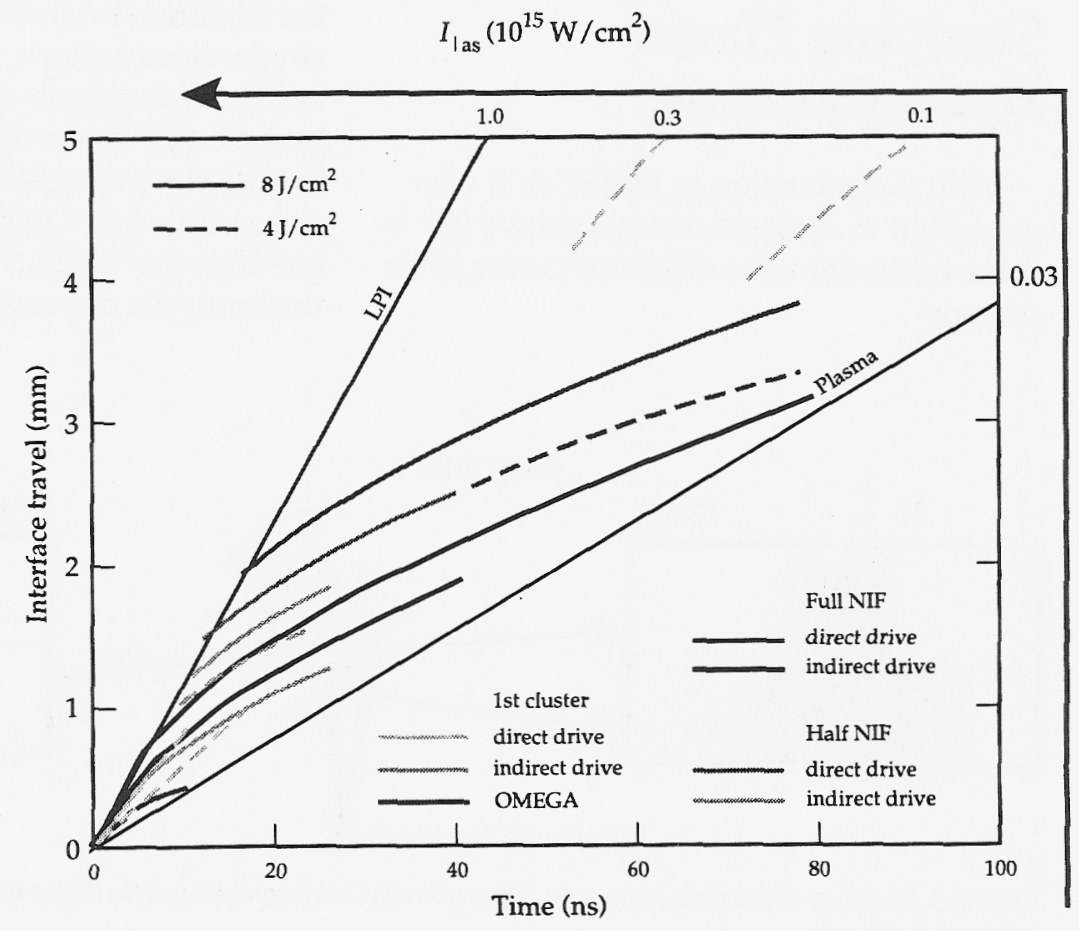




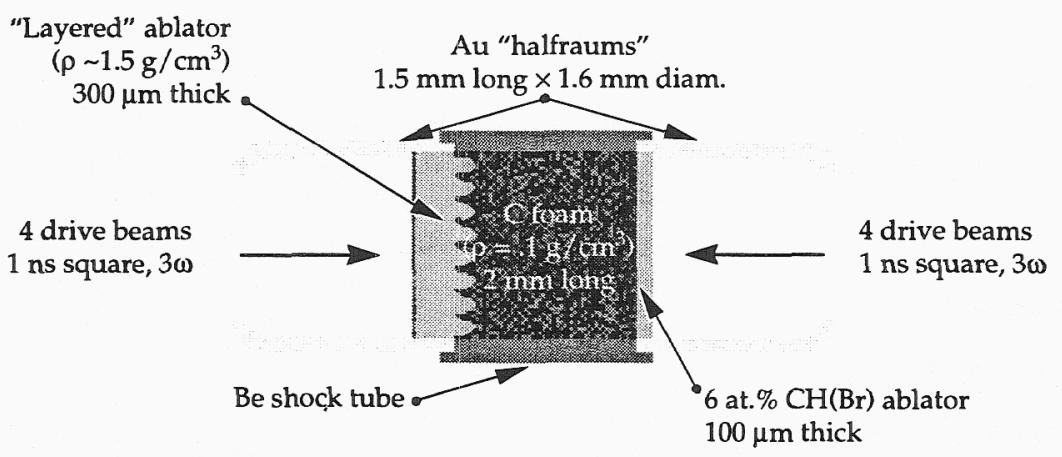

FIGURE 5. Experimental geometry. Four drive beams from Nova drive each end of the shock tube. An additional two Nova drive beams are used for backlighting. (NIF-0101-00017) the ripples at the interface to grow via the Richtmyer-Meshkov instability. A second, counterpropagating shock was launched from the opposite end of the shock tube by a second half-hohlraum driver that traversed the developing mix region at some later time. This unique geometry allowed independent control of the relative timing of the two shocks and their relative strength.

The experimental configuration is illustrated in Figure 5. The quality of the data obtained with this target was greatly improved over prior experiments by the use of a layered ablator. The perturbed ablator was constructed by using two density-matched plastic materials, only one of which was radiographically opaque to the backlighter $\mathrm{x}$-rays. The opaque material was confined to the central 100 microns along the line-of-sight, thus virtually eliminating the complications due to shock curvature in that direction. The initial perturbation was a $100-\mu \mathrm{m}$ wavelength ripple with an initial amplitude of $1 \mu \mathrm{m}$.

Two experimental results are shown in Figure 6. In the one at left, the first shock has crossed the rippled interface, but the second shock has not yet impacted the perturbed interface. The right image is from later in the experiment after the passage of the second shock through the mixing layer. Initial comparisons of the measurements with two-dimensional radiation-hydrodynamics simulations performed with the
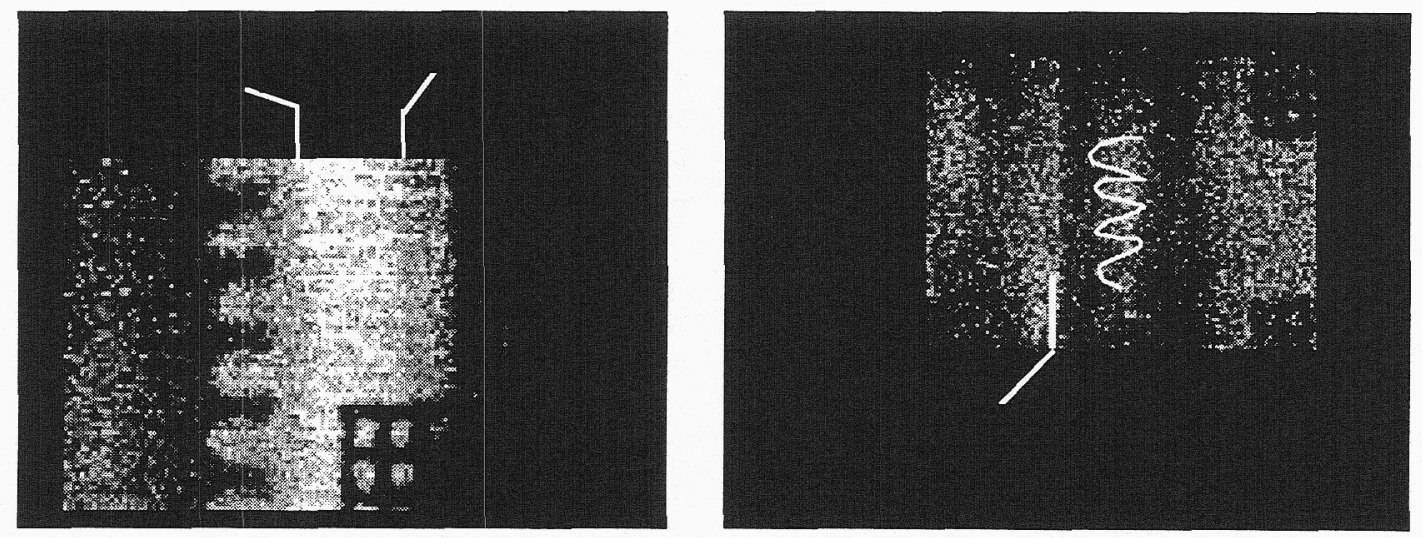

FIGURE 6. Backlit $x$-ray images of the instability growth. In the left image, the first shock at the interface has just propagated past the interface, and the second shock is moving toward the interface in the opposite direction. In the right image, the second shock has propagated past the interface. The instability growth at the interface is highlighted. (NIF-0101-00018) 
CALE code show excellent agreement (Figure 7). The drive conditions were varied in the calculations and experiments shown, with a corresponding difference in the observed mix width.

\section{Obtaining Images of Features on Nova with a New Gated Direct-Write CCD X-Ray Pinhole Camera}

One of the final new diagnostics to be fielded on the Nova laser was a new gated direct-write charge-coupled device (CCD) $\mathrm{x}$-ray pinhole camera that could be fielded in a six-inch manipulator (SIM) tube. A direct-write $x$-ray $C C D$ camera has several advantages over recording on $x$-ray film. A CCD device is inherently a linear device allowing more quantitative information to be obtained from an image. With a CCD device, the data are available immediately after the shot. Film, on the other hand, must be developed and then digitized before the data are available to the experimenter. A $C C D$ is inherently more sensitive than film and, with proper cooling, has superior signal to noise compared to film and comparable dynamic range. $X$-ray CCDs

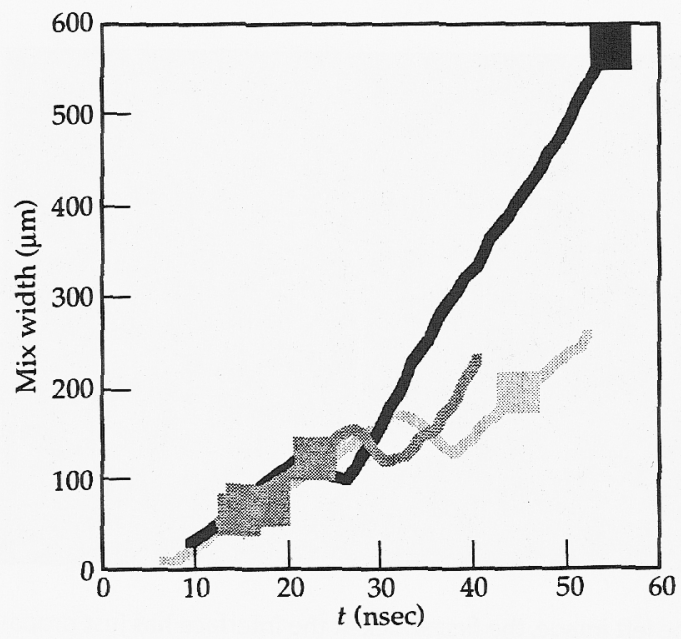

FIGURE 7. Experimental measurements of mix width from single and double shocks compared to CALE simulations. The solid lines are from three CALE simulations with differing drives; the data are shown as squares. (NIF-0101-00019) are capable of detecting individual $x$-ray photons. Fielding a CCD camera is more complex than fielding a piece of $x$-ray film, so one of the primary reasons for developing this camera in the last days of Nova was to gain experience with this type of detector, as it is expected that CCDs will nearly completely replace film for diagnostics on NIF. It is expected that this camera, once operational, will be relocated and used on OMEGA.

This camera used a $1 \mathrm{~K} \times 1 \mathrm{~K}$ backthinned $C C D$ chip and was operated with limited cooling. The lack of cooling meant that backgrounds were rather high, which reduced the dynamic range of the instrument. For use at the OMEGA laser and on the NIF, water cooling lines will be available allowing much lower backgrounds.

Figure 8 is a picture of the SIM-based $x$-ray CCD imager fielded on Nova in FY99. The CCD is in the square frame of aluminum towards the lower left of the picture. The camera was demonstrated to be operational and fielded on several shots prior to the closure of Nova.

\section{The Linear Rayleigh- Taylor Growth for Ablatively Driven Al on Nova}

The RT instability, which occurs when a lower-density fluid accelerates its higherdensity neighbor, is common in nature. At an ablation front a sharp reduction in the growth rate of the instability at short wavelengths can occur, in marked contrast to the classical case in which growth rates are highest at the shortest wavelengths. Theoretical and numerical investigations of the ablative Rayleigh-Taylor instability are numerous and differ considerably on the level of stabilization expected. We have completed a series of laser experiments designed to probe the rollover and cutoff region of the ablation-front $\mathrm{RT}$ dispersion curve in indirect drive.

Aluminum foils with imposed sinusoidal perturbations ranging in wavelength from 10 to $70 \mu \mathrm{m}$ were ablatively accelerated with a radiation drive generated in a gold cylindrical hohlraum (Figure 9). A strong shock wave compresses the package 


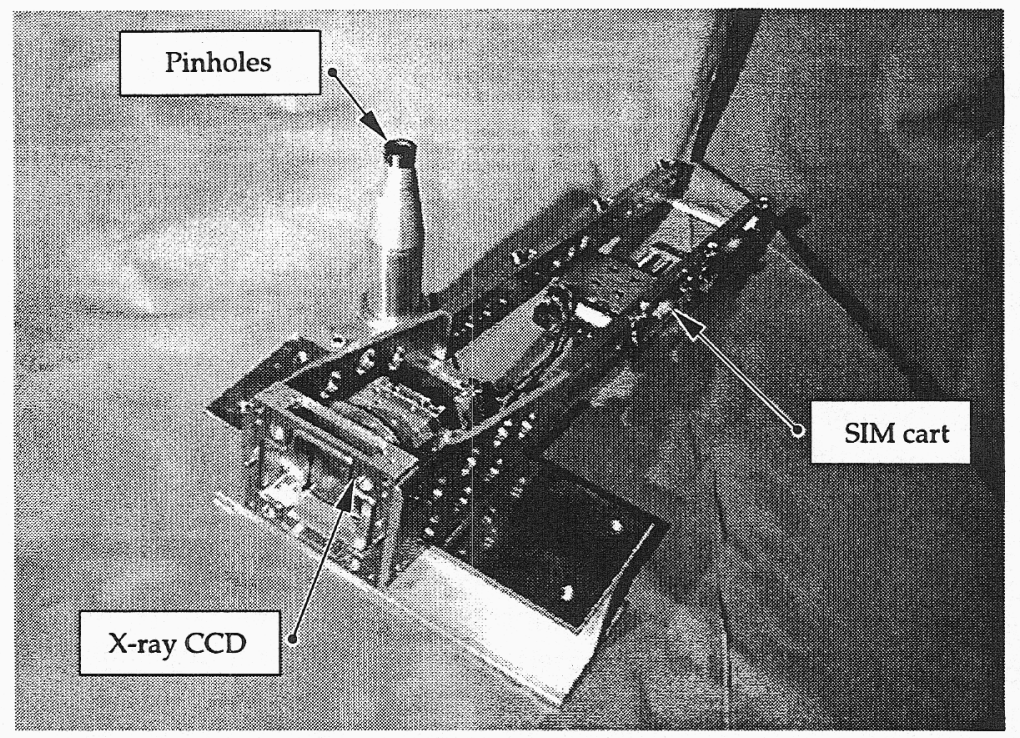

FIGURE 8. SIM-based $x$-ray CCD imager. The CCD is mounted in the back of the SIM cart. The snout that holds the pinholes is normally mounted on the front of the cart. (NIF-0101-00020)

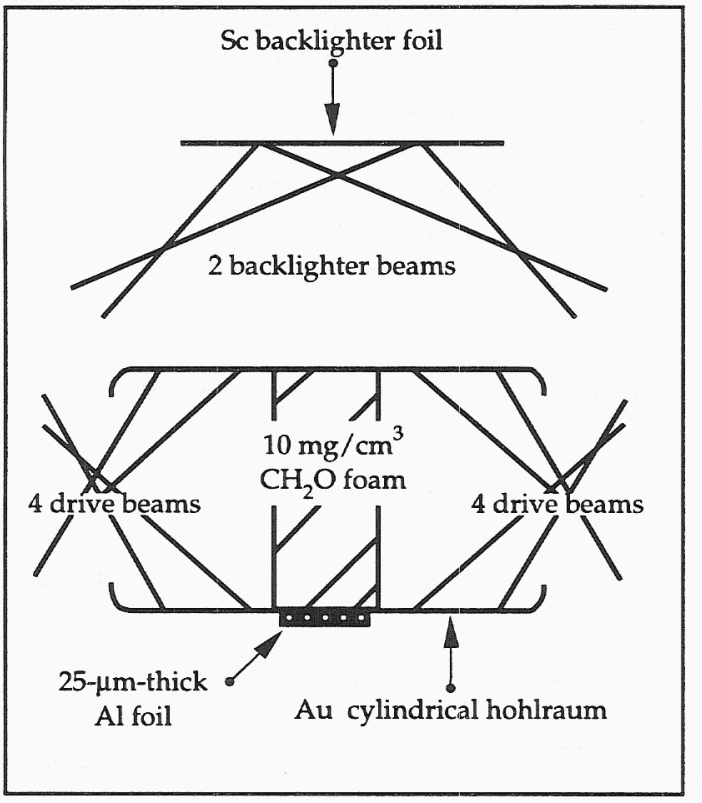

FIGURE 9. Experimental geometry. Eight drive beams from Nova illuminate the hohlraum. Two Nova beams illuminate a backlighter. $\quad(40-00-1100-6312 \mathrm{pb} 01)$

followed by an $\sim 2$-ns period of roughly constant acceleration, and the experiment is diagnosed via face-on radiography. Perturbations with wavelengths $\sim 20 \mu \mathrm{m}$ experienced substantial growth during the acceleration phase, while shorter wavelengths showed a sharp drop-off in overall growth. These experimental results compared favorably to LASNEX calculations

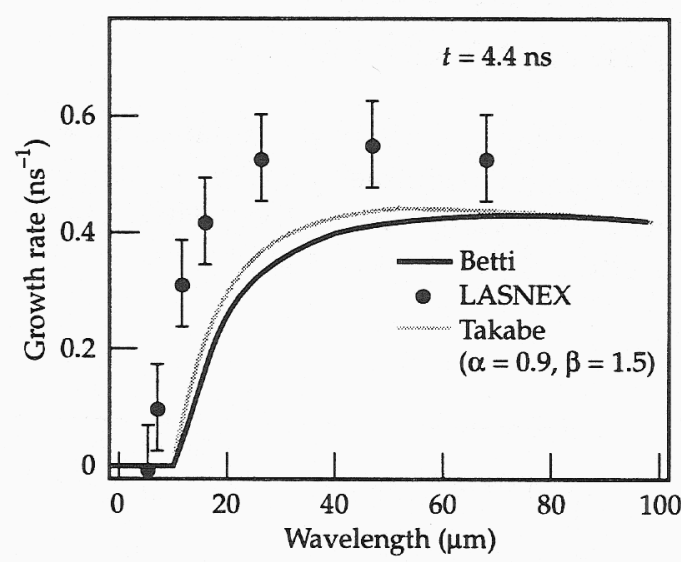

FiguRE 10. Comparison of dispersion curves for the ablatively stabilized Rayleigh-Taylor instability at 4.4 nsec. Calculations are compared with the Takabe-Morse model and Betti models. (40-00-1100-6313pb01)

(Figure 10); however, the growth is significantly affected by the rippled shock launched by the drive.

We performed numerical simulations that minimized the influence of the rippled shock wave by only imposing the perturbation at the ablation front after the shock had broken out of the rear surface of the target. This mitigates the effect of shock transit on the eventual growth of the perturbations, allowing comparisons to the analytic model developed by R. Betti and colleagues ${ }^{2}$ at the University of Rochester. This first-principles 
model uses one-dimensional density and pressure profiles from simulations as input to calculations of the perturbation growth rate. The agreement between the numerical experiment and the Betti model is quite good, particularly in predicting the location of the rollover in the dispersion curve. Figure 11 shows the simulated result and Betti prediction. We have also included the result of a Takabe fit to the simulations result. Here the coefficient in front of the stabilizing term in the growth rate equation is treated as a free parameter, thus assuring good agreement with the simulation.

This combination of experiments, simulations, and analytic modeling illustrates the qualitative simplicity yet quantitative complexity of the compressible RT instability.

\section{Supersonic Radiation Flow on OMEGA}

Detailed experimental radiation flow campaigns in simple cylindrical geometries were completed at the OMEGA laser facility. $^{3,4}$ The goal was to produce a reproducible testbed for supersonic radiative heating of low- and high- $Z$ foam disks of varying diffusivity. A new axisymmetric cylindrical hohlraum and package geometry amenable to integrated 2D modeling was successfully utilized. The $x$-ray drive was up to 2.4 ns long, with a measured peak temperature of $190 \mathrm{eV}$, irradiating 1.6-mm-diameter foam packages. Fully supersonic (radiation $\mathrm{Mach} \# \geq 3$ ) radiation flow was studied for both the weakly diffusive case of $50 \mathrm{mg} / \mathrm{cm}^{3} \mathrm{SiO}_{2}$ foam and the more strongly diffusive case of $40 \mathrm{mg} / \mathrm{cm}^{3} \mathrm{Ta}_{2} \mathrm{O}_{5}$ foam. The breakout of the radiation wave was observed by soft $x$-ray gated and streaked imaging diagnostics x-rays operating at $h v=550 \mathrm{eV}$.

Figure 12 shows examples of the clean radially and temporally resolved radiation breakout data obtained for $\mathrm{Ta}_{2} \mathrm{O}_{5}$ foams of varying lengths encased in Au cylindrical tubes. The data is reproducible to the $10 \%$ level in radiation breakout delay. The predicted number of mean free paths ( $\mathrm{mfp}$ ) at breakout was up to 4 for the longest $\mathrm{Ta}_{2} \mathrm{O}_{5}$ foam and $\approx 1 \mathrm{mfp}$ for the $\mathrm{SiO}_{2}$ foams. The radiation breakout delays and strengths
Figure 11. Comparisons of experimental data and calculations. No growth is observed at $10-\mu \mathrm{m}$ and $12-\mu \mathrm{m}$ wavelengths, while growth is observed at longer wavelengths. This demonstrates the cutoff in the Rayleigh-Taylor instability due to ablative stabilization. (40-00-1100-6314pb01)

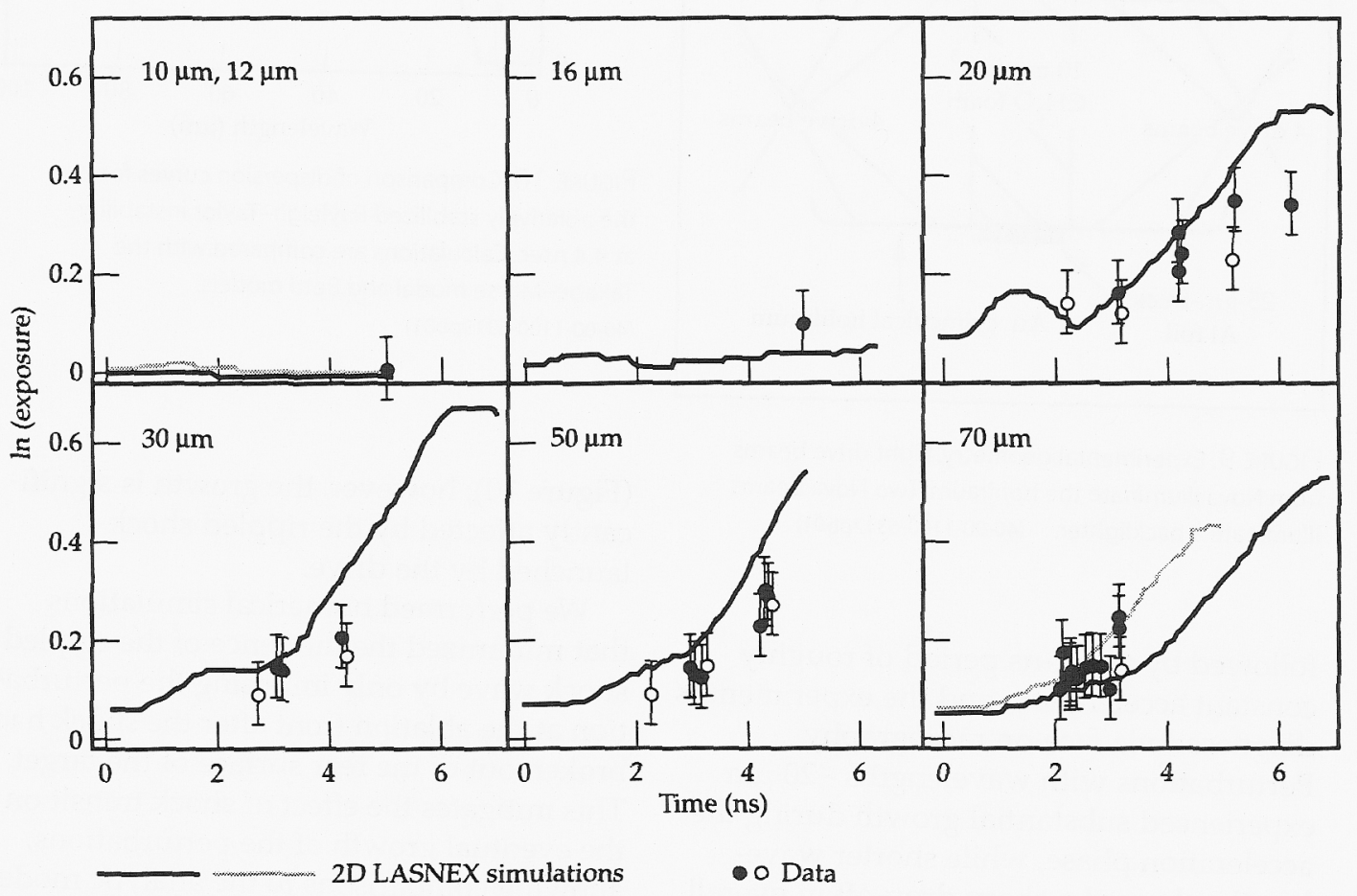


are compared to simulations in Figure 13. Best agreement is found by using the more sophisticated and applicable opacity models (OPAL rather than XSN for $\mathrm{SiO}_{2}$ ). Nevertheless, the simulations still predict slightly earlier breakout times than measured. This discrepancy has been recently explained by the lower-than-predicted $x$-ray drive temperatures measured during the first $1 \mathrm{~ns}$ of the drive.

The data in Figure 12 also show a lag in the radiation breakout at the edges for the the longer foam cases. This lag is attributed to radiative losses to the gold walls. The results show more radiation front curvature for the more diffusive $\mathrm{Ta}_{2} \mathrm{O}_{5}$ case as expected. Simple analytic estimates suggest losses to the wall will dominate for tube aspect ratios (axial length/diameter) $>1$ at OMEGA-size facilities. Hence, new schemes in which the wall is externally heated have been proposed for increasing the aspect ratio of future radiation flow experiments.

\section{Notes and References}

1. D. E. Grady and J. R. Asay, J. Appl. Phys. 53, 7350 (1982).

2. R. Betti et al., Phys. Plasmas 3(5), 2122 (1996).

3. C. A. Back et al. Phys. Rev. Lett., 2, 51 (2000).

4. C. A. Back et al., Phys. Plasmas 7(5 PT2): 2126-2134).

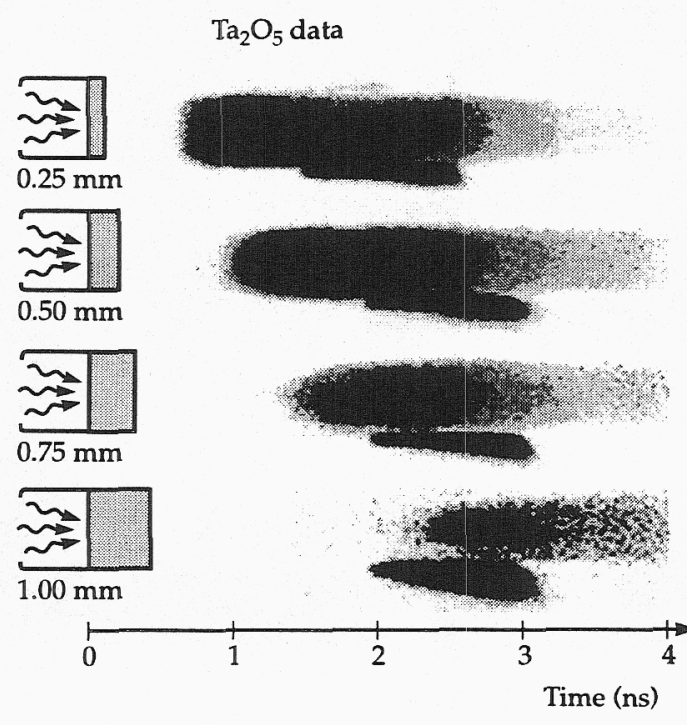

FIGURE 12. Images of the series of $\mathrm{Ta}_{2} \mathrm{O}_{5}$ data. Four different lengths span a range of 1.2 to 3.8 mean free paths. The radiation front breakout seen at the left-hand side of the data becomes more curved for longer lengths as the energy lost to the wall becomes more significant. (08-00-0300-0774pb01)

\section{Off-Hugoniot Deuterium EOS Experiments on Nova}

Measurements of the equation of state (EOS) of deuterium at megabar pressures on Nova ${ }^{1-4}$ have led to a reconsideration of the theory of hydrogen isotopes at high pressure and finite temperature. The experiments involved driving a strong shock into a cryogenic deuterium sample and determining conditions in the shocked state through measurements of the resulting fluid motion. A single beam of the Nova laser irradiating the target at normal incidence was used to drive the shock. The Nova data showed that the Hugoniot was much more compressible between 0.3 and 3.5 Mbar than had been anticipated by most EOS calculations. The Hugoniot data included pressure, density, and temperature. All data were consistent with the
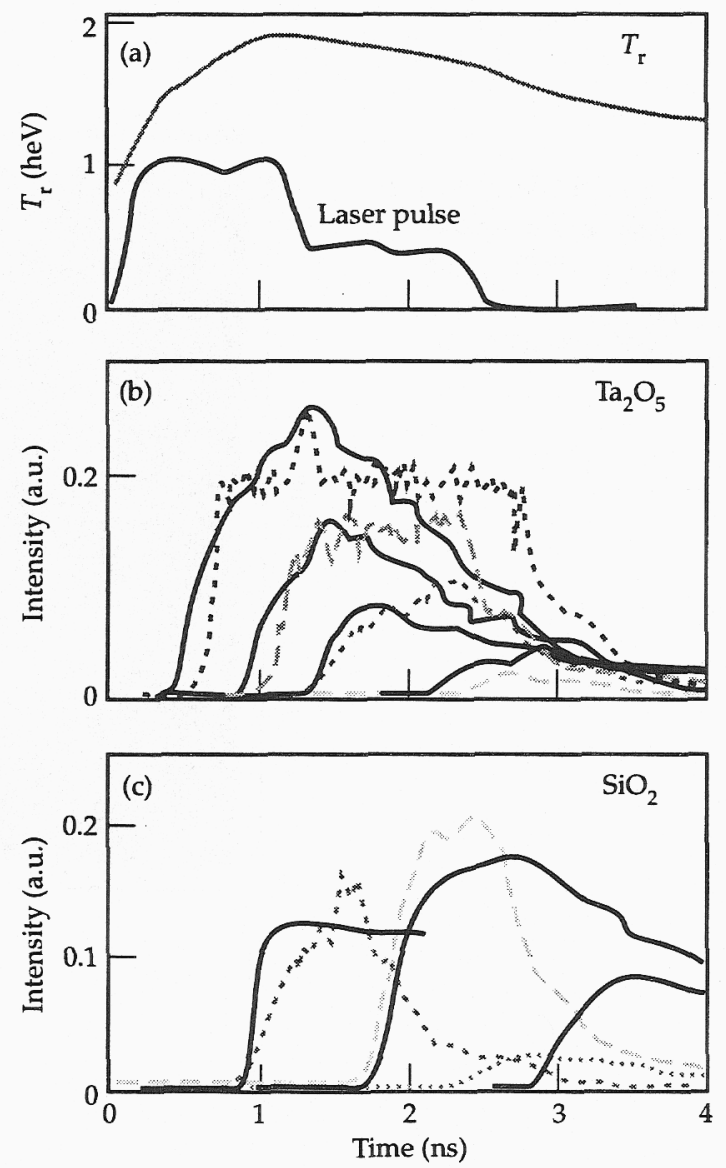

FIGURE 13. (a) The measured radiation drive temperature history plotted along with the normalized laser pulse. (b) Temporal intensity lineouts of the $\mathrm{Ta}_{2} \mathrm{O}_{5}$ data at foam center. Dashed lines show data; solid lines show calculations. Each pair corresponds to a differentlength foam: $0.25 \mathrm{~mm}$, $0.50 \mathrm{~mm}, 0.75 \mathrm{~mm}$, and $1.00 \mathrm{~mm}$. (c) Temporal intensity lineouts of the $\mathrm{SiO}_{2}$ data at foam center. Dashed lines show data; solid lines show calculations. Foam lengths were $0.5 \mathrm{~mm}, 1.0 \mathrm{~mm}$, and $1.25 \mathrm{~mm}$.

(08-00-0300-0775pb01) 
occurrence of a dissociative phase transition between the low-pressure insulating state and a higher-pressure conducting state. Moreover, measurements of the 1064-nm reflectance of the shock front using a laser probe indicated that the transition was continuous.

The EOS model of Saumon and Chabrier, widely used in astrophysical models of giant planets and cool stars, predicts a first-order phase transition from insulating to metallic states just to the compressive side of the Hugoniot. ${ }^{5}$ This is a regime accessible using two shocks, a socalled reshock experiment.

An experiment was carried out that used Nova to drive a shock into deuterium $\left(D_{2}\right)$ contained between an aluminum (Al) pusher and a lithium-fluoride $(\mathrm{LiF})$ window. When the shock in the $D_{2}$ rebounded off of the $\mathrm{LiF}$, the $\mathrm{D}_{2}$ was reshocked to a higher pressure. These experiments required that several beams be used at a $55^{\circ}$ angle of incidence. This is too steep to reliably propagate a steady uniform shock, so an indirect-drive half-hohlraum was employed. A velocity interferometer (VISAR) was used to measure shock speed and determine reflectance.

Figure 14 shows a streak VISAR interferogram of a reshock viewed through the $\mathrm{LiF}$ window. Time goes upward in the figure. Reflections occur (chronologically) from the Al pusher, then from the front of an attenuating shock in $\mathrm{D}_{2}$, then from the shock front in the LiF. The evaluation of the final state of the reshocked $D_{2}$ depends on knowing the EOS of the shocked LiF in a regime where the LiF EOS is not well known. Although the EOS data are still undergoing evaluation, the $\mathrm{D}_{2}$ is observed to reshock from $\sim 0.25 \mathrm{Mbar}$ to $\sim 1 \mathrm{Mbar}$, which is consistent with the earlier Nova experiments.

\section{Notes and References}

1. L. B. Da Silva et al., Phys. Rev. Lett. 78, 483 (1997).

2. R. Cauble et al., Phys. Plasmas 4, 1857 (1997).

3. G. W. Collins et al., Phys. Plasmas 5, 1864 (1998).

4. G. W. Collins et al., Science 281, 1178 (1998).

5. D. Saumon and G. Chabrier, High Pressure Res. $16,331(2000)$.
FIGURE 14. VISAR interferogram of a $D_{2}$ reshock experiment. Time goes upward.

(40-00-1100-6101pbo1)
Reshock from $\mathrm{LiF}$ window

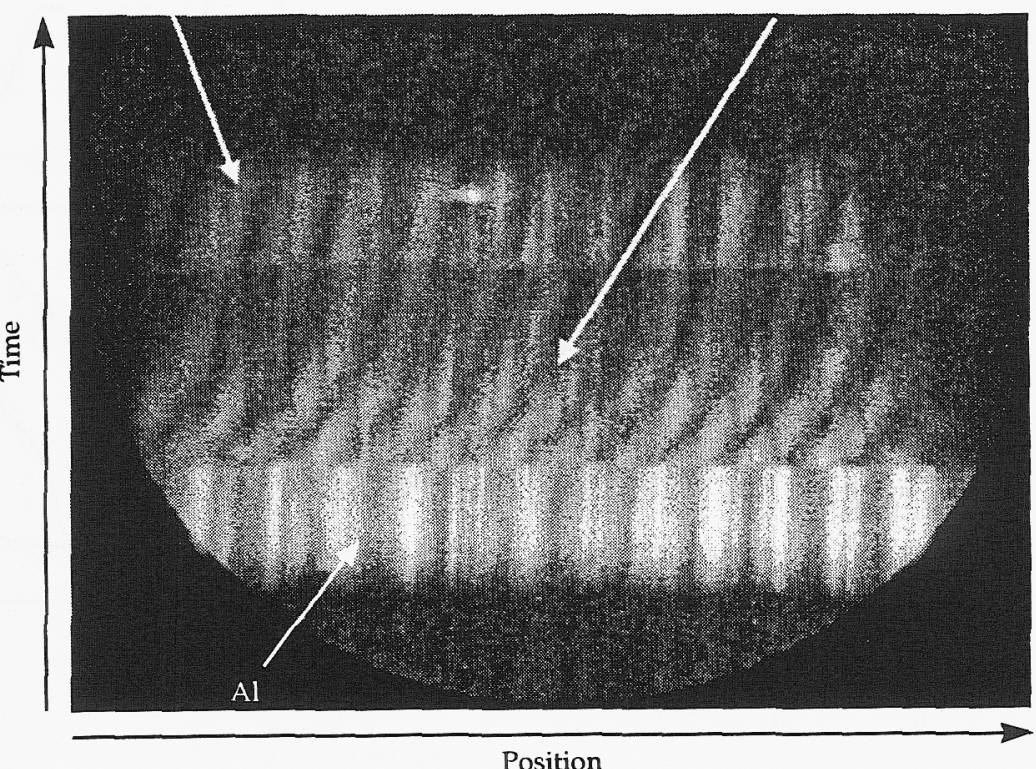




\subsection{TARget Development, Fabrication, AND HANDLING}

$\mathrm{T}$ he National Ignition Facility (NIF) capsule designs require us to meet very exacting specifications for capsule symmetry, surface finish, and material composition. All NIF capsule fabrication options except machined Be require a mandrel upon which the ablator is deposited. This mandrel sets the baseline sphericity of the final capsule, especially over the low modes. Subsequent coating operations may degrade the capsule surface finish but are unlikely to improve it. Thus our task is twofold: first, to prepare mandrels that meet or exceed final NIF capsule requirements and second, to develop ablator technologies that can be used to uniformly coat the mandrel to the desired thickness without deforming the underlying mandrel and without adding higher frequency roughness. During fiscal year 1999 (FY99), we continued our work on mandrel development, vapor-deposited polyimide ablator deposition, and sputtered Be ablator deposition.

In the sections that follow we will discuss our progress during FY99 in developing both mandrels and ablator technologies suitable for meeting the target design criteria. Much of this material has been published elsewhere in greater detail, and the reader is referred to the references given for additional detail. In the area of mandrels we have made significant progress, reducing the very low-mode asymmetry to levels that are below design specification, and we are making good progress in reducing the mode 10 to 100 roughness, though further improvement is required.
Our polyimide vapor deposition work has experienced both successes and disappointments. We have successfully produced capsules with a $150-\mu \mathrm{m}$-thick, uniform, polyimide ablator; however, the surface finish was very poor. Efforts to improve the surface finish through modifications of the coating process proved inadequate to meet NIF specifications; however, we have developed a very promising postdeposition process that markedly improves the surface finish, and this approach will be vigorously pursued in fiscal year 2000 (FY00). We also demonstrated that higher-strength polyimide films could be produced, but complications involved with the processing coupled with a decreased interest in strength as a key capsule requirement have caused us to terminate this line of research. Progress in the area of sputtered Be ablators has been slow. We have previously demonstrated that NIF thickness $\mathrm{Cu}$-doped Be ablators could be applied to plastic mandrels; however, surface finish did not meet NIF specifications, and the pathway to filling the capsules with DT was unclear. In the last year we have explored deposition of amorphous B-doped Be in an effort to improve surface finish and have concluded that this approach is not promising for capsule ablators. An alternative to producing a smooth capsule is a postprocessing polishing step. We report on the construction of such a facility at General Atomics, modeled after systems in place at Los Alamos National Laboratory (LANL). Finally, our results from diffusion-based Be-capsule fill experiments will be discussed. 


\section{Improving Surface Finish of B-Doped Be by Varying Deposition Conditions}

\section{B-Doped Be Capsule Development}

We have observed that the surface of continuously doped Be/B coatings on capsules is extremely rough and becomes rapidly rougher with increasing layer thickness. Experiments on temperaturecontrolled planar substrates have suggested that deposition temperature and intrinsic stress might be important factors in the formation of these surfaces. The most accessible process variable is the pressure of the Ar sputter gas. Experiments with stress disks have shown that increasing the gas pressure reduces (but does not eliminate) the intrinsic compressive stress in deposited films. We deposited $\mathrm{Be} / 15$ at. \% B coatings on $\mathrm{CH}$ mandrels using an Ar pressure of $23 \mathrm{~m}$ Torr, rather than the usual 3.75 mTorr. No significant change was observed in the film morphology. Based on these results, we discontinued coating experiments using the continuously doped Be/B alloy on capsules. We have continued to study possible sources of the rough surfaces observed on these capsules and currently believe that a combination of limited surface mobility and selfshadowing due to the isotropic depositing flux is primarily responsible. ${ }^{1-3} \mathrm{~A}$ short study was done using alternating layers of $B e$ and $B$ to improve the surface finish (Figure 1). Capsules with an 11- $\mu$ m-thick coating of $50 \mathrm{~nm} \mathrm{Be} / 5 \mathrm{~nm}$ B layers showed a dense, fibrous microstructure in cross section (Figure 2) and had a 35-nm rms surface finish (much better than the continuously doped material).

\section{New Alloy Development}

Our work on new alloys in FY99 focused on a detailed survey of the Be-B-Fe system. Although the high- $Z$ dopant required for Be capsules is normally presumed to be $\mathrm{Cu}$, other elements, such as Fe, could be substituted. Selected area diffraction (SAD) performed on transverse-electromagnetic (TEM) cross sections of films deposited on planar substrates showed that at $\sim 15$ at. $\%$ B and above, a glassy structure was achieved. These results were obtained with both 0 and 1 at. $\% \mathrm{Fe}$, with indications that the onset of the glassy phase occurred at lower B levels in the presence of Fe. In earlier work on $\mathrm{Cu}$-doped $\mathrm{Be} / \mathrm{B}$, there was

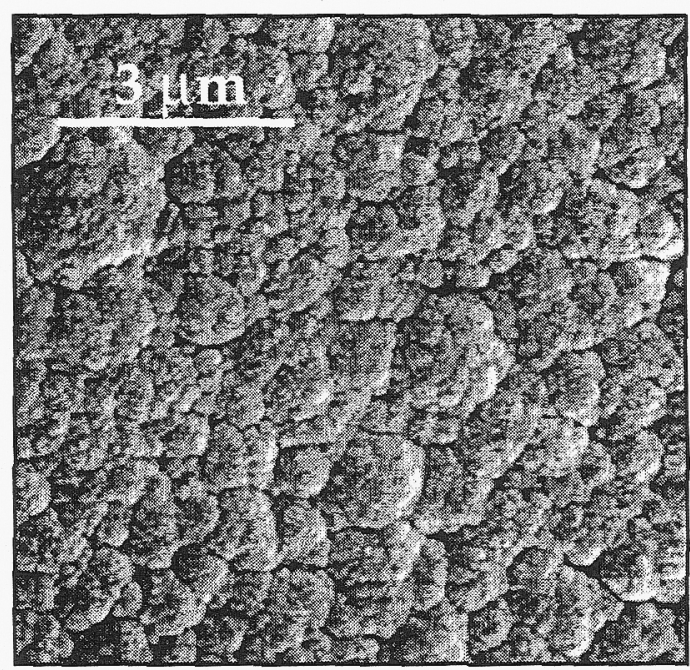

FIGURE 1. An 11- $\mu \mathrm{m}$-thick capsule coating composed of $50-\mathrm{nm} \mathrm{Be} / 5-\mathrm{nm} B$ layers. The rms roughness is approximately $35 \mathrm{~nm}$. (40-00-1100-6102pb01)

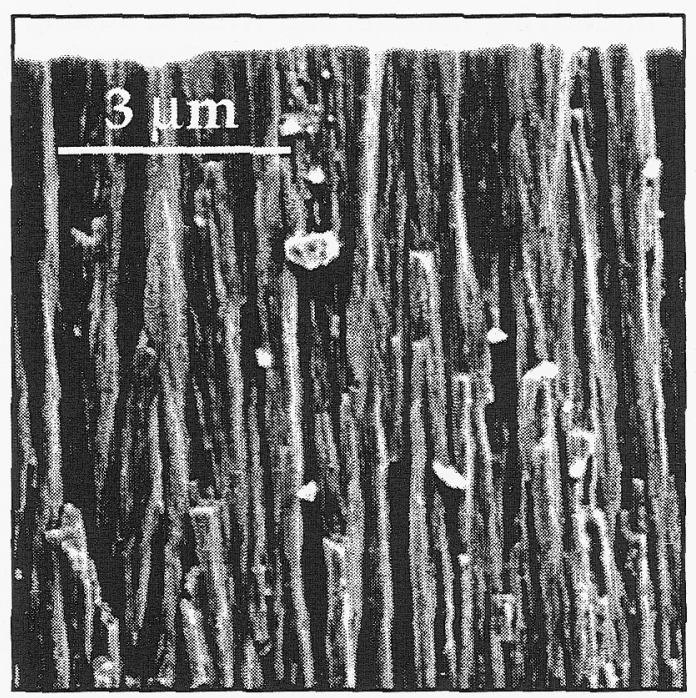

FIGURE 2. SEM fracture cross section of the Be/B multilayer film. Note the densely packed, refined grain structure. (40-00-1100-6103pb01) 
an apparent transition at about $11 \%$, as seen by scanning electron microscopy (SEM) and $x$-ray diffraction. SAD is a much more sensitive test, however, as it allows highly localized diffraction measurements. The $\mathrm{B}$ concentration was measured using Rutherford backscattering spectroscopy (RBS). Based on these results, a series of progressively thicker films (up to $\sim 60 \mu \mathrm{m}$ ) at a composition of 76 at. $\% \mathrm{Be}-23$ at. $\% \mathrm{~B}$ 0.9 at. $\% \mathrm{Fe}$ (316 stainless steel) were deposited on stationary capsules and flat substrates. To stay well above the onset of the amorphous phase, 23 at.\% B was chosen because there was concern that the higher substrate temperature of the capsules might tend to crystallize the coating. We found that thinner coatings were extremely smooth (a few $\mathrm{nm} \mathrm{rms}$ ), but that high levels of compressive stress caused failure in thicker films. Nevertheless, a 9- $\mu$ m-thick Be/23 at.\% B/0.9 at.\% Fe alloy was successfully deposited on moving $\mathrm{CH}$ capsules. The rms roughness of this coating was approximately $30 \mathrm{~nm}$, which is much higher than seen on flats. The morphology suggested that debris incorporation might be responsible for some of increased roughness.

\section{New Coating Geometries}

Our conclusion that isotropic deposition on capsules is largely responsible for the rough surfaces obtained in the B-doped Be capsule work has prompted us to investigate new coating geometries that might reduce this effect. Our first experiment was a test of a deposition geometry in which the capsules are coated from below through an aperture, which prevents the depositing flux from striking the surface at oblique angles. We made 2- to 3- $\mu \mathrm{m}$-thick coatings using $\mathrm{Be}$ and $\mathrm{Be} / 3$ at.\% $\mathrm{Cu}$ source targets. Compared to the conventional sputter-down procedure, the capsule surfaces are smoother $(\sim 10 \mathrm{~nm} \mathrm{rms})$ and have smaller grains $(\sim 100 \mathrm{~nm})$.

\section{Notes and References}

1. S. K. Dew, T. Smy, and M. J. Brett, J. Vac. Sci. Technol. B 10, 618 (1992).

2. J. A. Thornton, Ann. Rev. Mater. Sci. 7, 239 (1977).

3. R. Messier, A. P. Giri, and R. A. Roy, J. Vac. Sci. Technol. A 2, 500 (1984).

\section{Pd-Enhanced and High- Temperature Permeability of Hydrogen through Be}

\section{Low-Temperature Permeation}

During this year we completed our evaluation of a possible low-temperature permeation technique in which Pd layers on either side of a Be coating are used to enhance its permeability to hydrogen. We deposited $\mathrm{Pd}$ on a plastic mandrel, overcoated it with $33 \mu \mathrm{m}$ of $\mathrm{Cu}$-doped Be, and finished with another coating of $\mathrm{Pd}$. A cross section image of this coating is shown in Figure 3. These capsules were placed in $10 \mathrm{~atm}$ of $\mathrm{D}_{2}$ at $200^{\circ} \mathrm{C}$ for five days. Crush tests of five capsules revealed little or no fill. Based on these results, we have concluded that this technique is unlikely to succeed and have terminated these experiments.

\section{High-Temperature Permeation}

High-temperature permeability studies at General Atomics have focused on preventing oxidation of capsules during exposure to $D_{2}$ at high temperatures. Even a

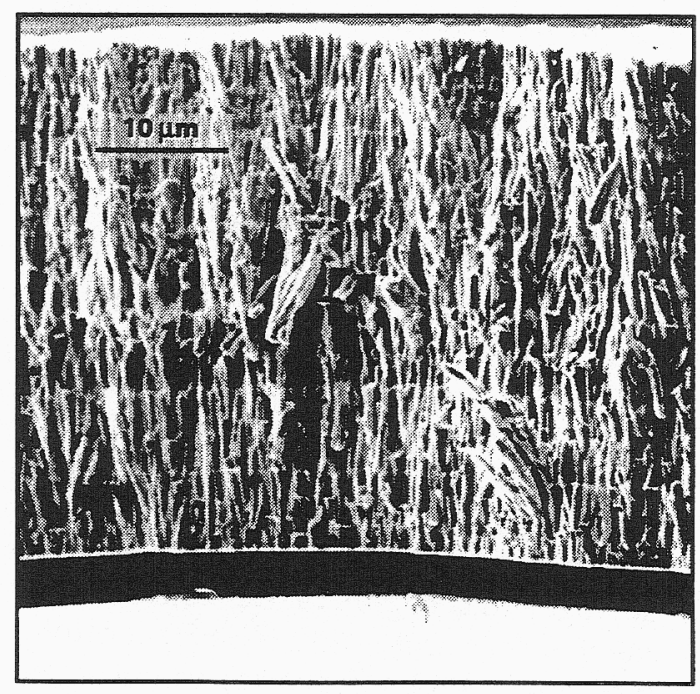

FIGURE 3. SEM cross section of a 33- $\mu$ m-thick Be coating on a 1-mm-diam $\mathrm{CH}$ mandrel. The light-colored bands are thin layers of $\mathrm{Pd}$ deposited to protect against oxidation between successive coating runs. (40-00-1100-6104pb01) 
very thin oxide layer drastically reduces permeability. Pd coatings have been used as an antioxidation barrier, but have not proven successful. Energy-dispersive $\boldsymbol{x}$-ray diffraction (EDX) and secondary ion mass spectrometer (SIMS) analyses have shown the presence of $\mathrm{Be}$ and $\mathrm{O}$ on the exterior surface of these capsules after exposure to $10 \mathrm{~atm}$ of $\mathrm{D}_{2}$ at $400^{\circ} \mathrm{C}$. Auger spectroscopy verified the presence of $\mathrm{BeO}$ at the surface. Sputter depth profiling demonstrated that the $\mathrm{Pd}$ had penetrated several $\mu \mathrm{m}$ into the bulk. A second experiment was performed in which 300-nm-thick layers of either $\mathrm{Au}, \mathrm{Pt}$, or $\mathrm{Pd}$ were deposited on capsules and their behavior compared under similar conditions ( $10 \mathrm{~atm}$ of $D_{2}$ for 5 days at $\left.400^{\circ} \mathrm{C}\right)$. In all three cases, the heated capsules gained weight $(6-8 \%)$, presumably from oxidation, and much of the noble metal was absorbed into the bulk of the coating. EDX once again confirmed the presence of $\mathrm{Be}$ and $\mathrm{O}$ at the surface.

\section{Effectiveness of Mechanical Polishing Techniques for Be Capsules}

During FY99 General Atomics was tasked to build a polishing apparatus suitable for use on Be capsules. A polishing system was designed based upon a working prototype at LANL and built using an outside vendor (see Figure 4). Briefly, the design involves two plates, each with a circular groove machined in the surface, that face each other and rotate. The rotation axes are offset, so the grooves intersect at two points. Figure 5 shows the groove in the lower plate. The shell is captured in one of these intersection points, and the motion of the plates forces it to tumble randomly in an abrasive slurry. The system has been installed at General Atomics, and testing using a Ti ball as a surrogate has begun. Significant environment, safety, and health (ES\&H) controls, including a high-efficiency particulate air (HEPA)-filtered fume hood, had to be put in place to enable Be work. Some minor additional ES\&H issues remain to be resolved prior to the start of Be operations.

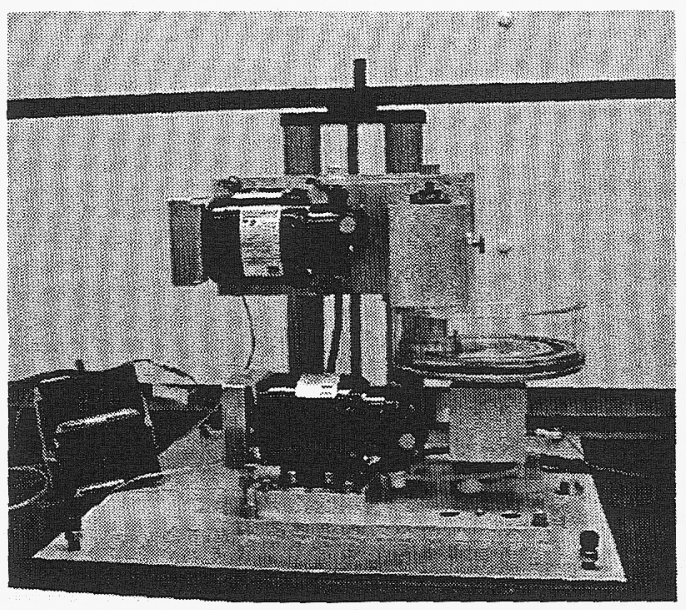

FIGURE 4. Beryllium capsule polishing apparatus. (40-00-1100-6105pb01)

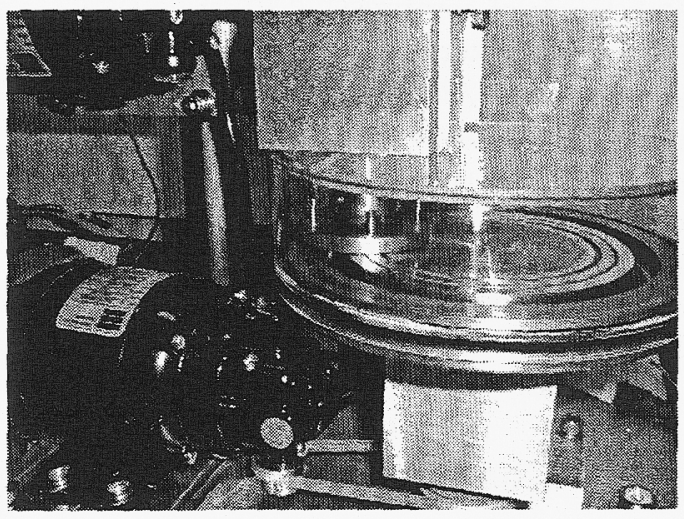

FIGURE 5. Close-up showing the polishing grooves. (40-00-1100-6106pb01)

\section{Polyimide Shells over $100 \mu \mathrm{m}$ Thick}

Over the past three years LLNL has been developing a vapor deposition approach for the fabrication of polyimide ablators for NIF-scale capsules. ${ }^{1,2}$ As reported in the FY98 annual report, our approach is to vapor deposit the two monomeric precursors of Kapton ${ }^{\mathrm{TM}}$, 4,4'-oxydianiline (ODA) and pyromellitic dianhydride (PMDA), on to spherical plastic mandrels in a bounce pan. Upon deposition, there is some reaction to form amic acids oligomers; subsequent thermal processing of the coated capsule converts the 
coating to polyimide. The process is shown schematically in Figure 6. In FY98 we focused primarily on developing deposition apparatus and technique; a major focus was controlling the deposition stoichiometry. At the end of FY98 and throughout FY99, we have focused on deposition on capsule mandrels. We have found that vapor deposition coatings of as much as $160 \mu \mathrm{m}$ can be deposited on mandrels and thermally cured to produce NIF-scale polyimide ablators. Cross-sectional views of these ablators by SEM indicate uniform, defect-free walls; however, the surface finish of the capsules after deposition was in general very poor and remained unchanged during the thermal conversion of the as-deposited coating to polyimide. Thus a major focus of our work during FY99 has been the determination of the cause of this roughness and the testing of methods to reduce it.

The most likely source of this roughness was abrasion associated with capsule/ bounce-pan interaction. This was confirmed through experiments that demonstrated that the coating roughness increased both with initial mandrel mass and coating time. ${ }^{3}$ In the first experiments, we used both solid bead and hollow mandrels with different wall thicknesses to test the effect of mandrel mass, which should be related to the magnitude of the man$\mathrm{drel} / \mathrm{pan}$ interaction. We found that coatings were markedly rougher on heavier mandrels. In a second set of experiments, we found that roughness increased with coating time. In this case the effect could have been from the slowly increasing mass and coating thickness or total time in pan contact. However, we also showed that coatings of the same thickness produced in shorter times by increasing the coating rate were smoother than those produced over longer times with slower coating rates. Thus it would appear that surface finish degrades as a function of time in the coater, consistent with an abrasion model as the source of the surface finish degradation. Nanoindentation experiments on the as-deposited coatings showed that the coatings had only one-half the hardness of typical plasma polymer coatings and only one-seventh as large a Young's modulus. These results are consistent with the abrasion model.

Our next approach was to attempt to modify the bounce-pan agitation to reduce the abrasive nature of the process. Several "gentler" pan agitation approaches were tested, including the use of a tilted, rotating pan that caused the shells to roll rather than bounce. Although gentler agitation in general produced somewhat improved surfaces, the degree of improvement was far from adequate to produce shells with surfaces approaching those required for the NIF target designs. Thus we concluded that noncontact coating methods and/or postprocessing was necessary if polyimide ablator capsules meeting NIF specifications are to be produced.

At the end of FY99 we began to explore these approaches. A vapor deposition chamber was modified by replacing the bounce pan with a gas-jet apparatus for shell levitation, thus eliminating the

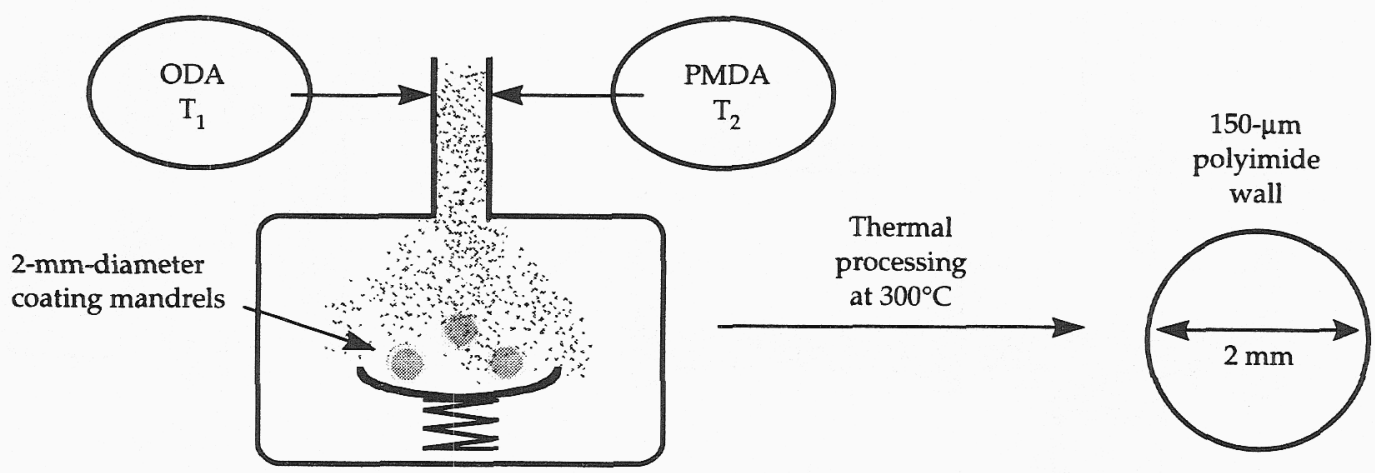

FIGURE 6. Schematic of the polyimide shell fabrication process. (40-00-1100-6107pb01) 
shell-pan contact. Our initial concern with this approach was that the presence of the levitating gas flow would compromise the high-vacuum coating conditions of our previous work, in the worst case preventing the monomer fluxes from reaching the shell. However, preliminary experiments showed that the gas flows necessary for levitation have an insignificant effect on the chamber pressure, and in fact coatings could be applied to the shell at a rate equal to about $80 \%$ of the rate obtained in bounce-pan coatings. Both levitation stability and control of shell rotation to obtain uniform coatings are areas that need to be further developed in FY00.

A novel technique for postdeposition smoothing shown in Figure 7 was developed in late FY99. We have found that we can levitate and significantly smooth a fully coated (but not imidized) shell in an air flow saturated with dimethyl sulfoxide (DMSO) and, after it has smoothed, raise the temperature of the levitating gas to $300^{\circ} \mathrm{C}$ to imidize the coating in situ. The mechanism, and related optimization, will be pursued fully in FYO0; however, it appears that the solvent vapors are absorbed into the surface layer of the capsule allowing at least the high-frequency morphology to flow, producing a locally smooth surface. The results of one of the preliminary experiments are shown in Figure 8. On the left is an optical photo of the surface of a roughly 50- $\mu \mathrm{m}$-thick asdeposited coating, and on the right is the same coating after smoothing and imidization. WYCO interferometric surface-finish measurements (94- $\times 123-\mu \mathrm{m}$ patch) show a reduction in the root mean square (rms) surface finish from 712 to $14.4 \mathrm{~nm}$.

\section{Notes and References}

1. C. C. Roberts et al., Fusion Technol. 35, 138 (1999).

2. C. C. Roberts et al., Fusion Technol. (2000), in press.

3. Ibid.

\section{Vapor Depositions of Higher-Strength Polyimide Formulations}

During FY99 we investigated the vapor deposition of a higher-strength polyimide coating. Polyimide shells require a tensile strength of $120 \mathrm{MPa}$ to hold the 360 -atm room temperature DT fill that is needed for an 80 - $\mu$ m-thick cryo DT layer. ${ }^{1}$ As reported in the FY98 ICF Annual Report, the vapor deposition of 4,4'-oxydianiline (ODA) and pyromellitic dianhydride (PMDA), the precursors to Kapton ${ }^{\mathrm{TM}}$,
FIGURE 7. Apparatus for smoothing polyimidemonomer-coated shells. (40-00-1100-6108pb01)

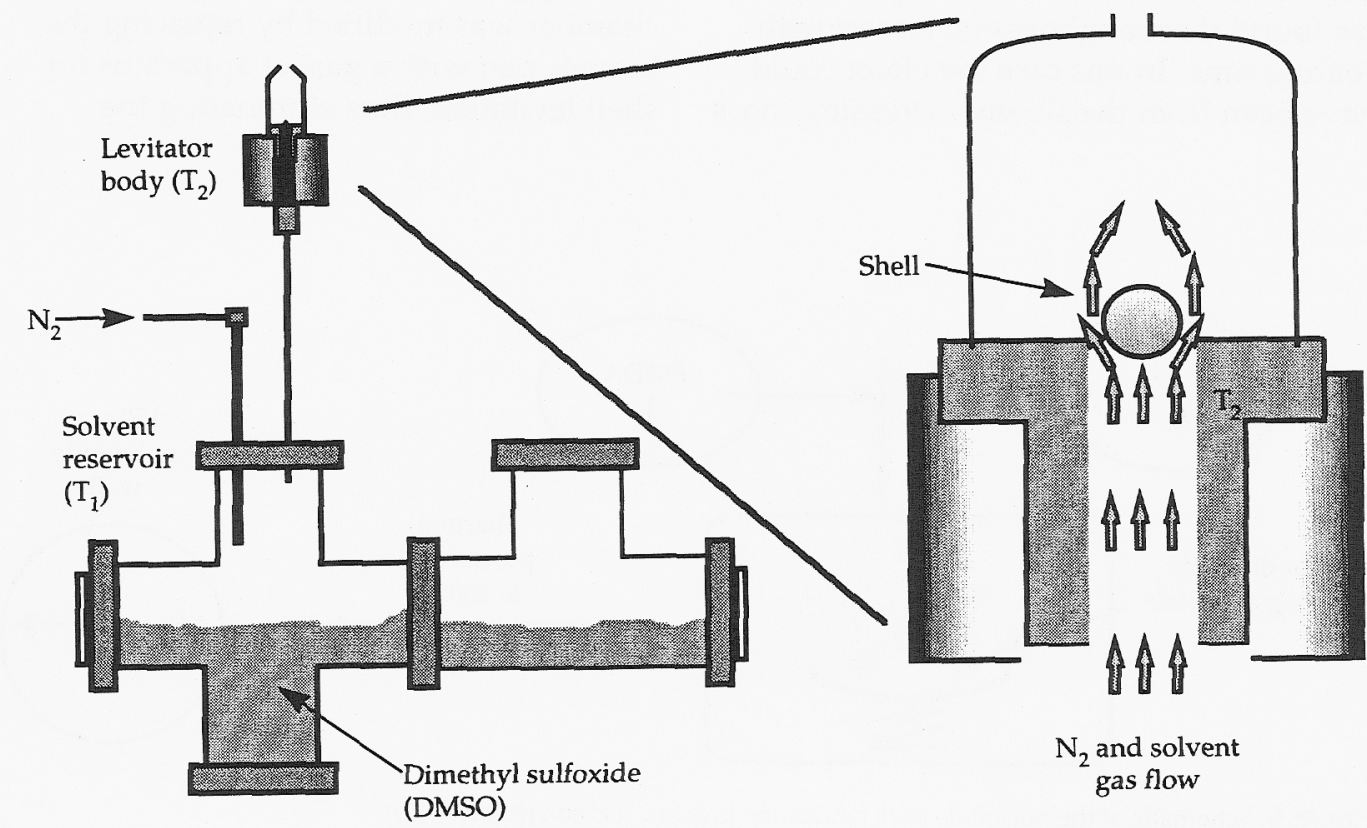




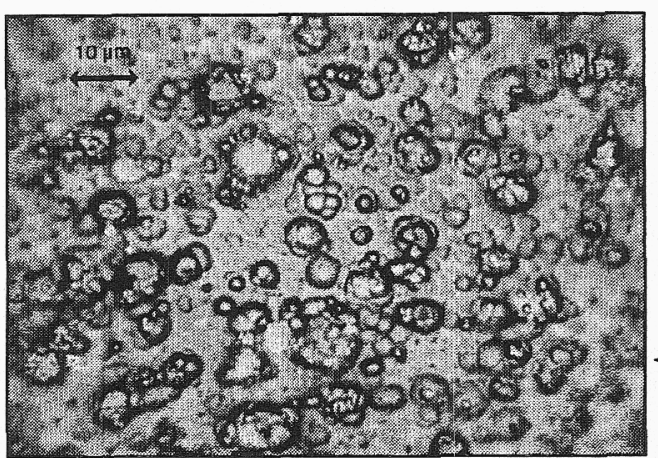

Before treatment

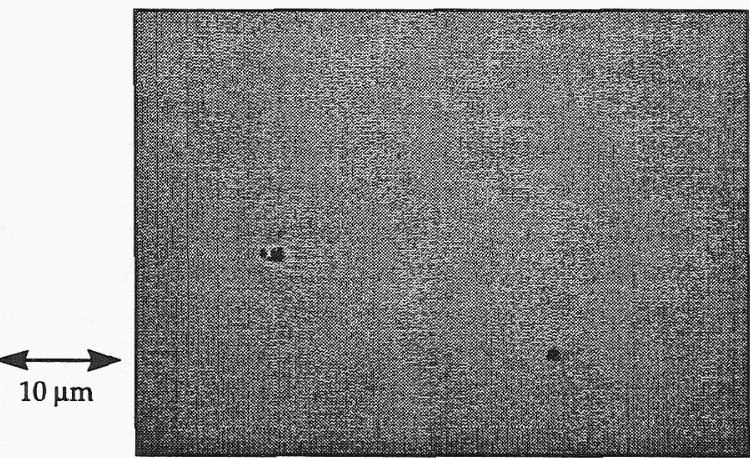

After treatment and curing results in a material with a tensile strength of about $100 \mathrm{MPa}$, a value that is about $40 \%$ of the $240 \mathrm{MPa}$ obtained for solutionprepared material. A higher-strength polyimide, Upilex ${ }^{\mathrm{TM}}$ (Ube Chemical Co.), has a reported tensile strength of $335 \mathrm{MPa}$. If the vapor-deposited material could again achieve $40 \%$ of the reported tensile strength, this would be $135 \mathrm{MPa}$ or slightly above the minimum needed for room-temperature transport. Upilex ${ }^{\mathrm{TM}}$ is made from p-phenylene diamine (PDA) and 3, $3^{\prime}, 4,4^{\prime}$ biphenyltetracarboxylic dianhydride (BPDA). The first thin vapor-deposited films were reported by Malba, Liberman, and Bernhard $t^{2}$ working at LLNL. Our objective was to deposit thicker films using the Upilex ${ }^{\mathrm{TM}}$ chemistry and make strength measurements to determine if this polyimide formulation might offer strength advantages over the Kapton ${ }^{\mathrm{TM}}$ chemistry described in the previous section.

Our first step was to determine the temperature-dependent sublimation rates of the two monomers so that conditions of equal molar deposition could be achieved. The mass loss from the individual evaporators was measured as a function of temperature. We found that BPDA was considerably less volatile than either of the Kapton ${ }^{\mathrm{TM}}$ monomers and required a temperature of at least $235^{\circ} \mathrm{C}$ to achieve an adequate deposition rate. However, to obtain an equimolar flux of PDA only required a temperature of about $63^{\circ} \mathrm{C}$. This required difference in the sublimation temperatures of the two monomers is in stark contrast to the situation for the ODA/PMDA depositions discussed above, where the temperatures are typically 147 and $156^{\circ} \mathrm{C}$, respectively. The widely differing evaporator temperatures for PDA/BPDA deposition require several modifications to assure that the low-temperature evaporator was not radiatively or conductively heated by the higher-temperature evaporator.

In another test, the deposition rate of each monomer was monitored in the vacuum chamber using a quartz crystal microbalance. We found that PDA showed no deposition as a single species, even though it was clearly leaving the evaporator based on mass loss measurements. We believe that PDA rapidly sublimes from the microbalance surface at the $10^{-5}$ Torr chamber pressure. However, when a reactive species such as BPDA is present, some fraction of the PDA reacts to form polymer and is retained in the growing film rather than being reemitted.

Initial experiments with BPDA/PDA deposition started with a goal of balancing the stoichiometry based on evaporator mass loss measurements to achieve a highmolecular-weight, high-strength polymer. The films that were formed were opaque, inhomogeneous, and showed no measurable strength. Because of the PDA volatility, we believe that these BPDA/PDA films were depleted in PDA. Thus a series of experiments were performed using a constant temperature for $\mathrm{BPDA}\left(235^{\circ} \mathrm{C}\right)$ and PDA evaporator temperatures from 60 to $105^{\circ} \mathrm{C}$ in order to increase the PDA flux. The films were deposited on glass substrates coated with a cesium iodide release layer. After thermal curing, the coated substrates were immersed in water to release
FIGURE 8. Representative optical photos (900x) of as-deposited (left) and solvent-treated (right) shell surfaces. The rms roughnesses determined by WrCO interferometry (94- $\times 123-\mu \mathrm{m}$ patch scans) are 712 and $14.4 \mathrm{~nm}$, respectively. (40-00-1100-6109pb01) 
the polyimide coating. The films were characterized for tensile strength using a pressure burst apparatus and for modulus and hardness using nanoindentation. We found that the tensile strength increased with increasing PDA flux up to a maximum and then declined. We believe this maximum represents the equimolar composition in the deposited film. The best vapor-deposited Upilex ${ }^{\mathrm{TM}}$ film had a tensile strength of $170 \mathrm{MPa}$ (abut $50 \%$ of the literature value) and was produced with a 25-35 molar excess of PDA. The measured modulus and hardness for vapor-deposited Upilex ${ }^{\mathrm{TM}}$ were 5.5 and $0.66 \mathrm{GPa}$ compared to 5.9 and $0.68 \mathrm{GPa}$ for commercial Upilex ${ }^{\mathrm{TM}}$.

The goal of achieving a tensile strength sufficient for room temperature transport was met. Unfortunately, the processing difficulties for the vapor-deposited Upilex ${ }^{\mathrm{TM}}$ material make it a very challenging material to consistently prepare. The large temperature difference between the two monomers requires the evaporators to be isolated to allow control of the lowertemperature evaporator. The high volatility of the PDA requires a large excess of this monomer that makes control of stoichiometry difficult. Further, the large quantity of PDA requires efficient trapping of the PDA to maintain a clean pumping system. Because of the difficulties in maintaining monomer balance and system cleanliness, coupled with the decreased importance of ablator strength for NIF capsules because of the planned cryo transport system, we have decided to focus our effort on the more controllable Kapton ${ }^{\mathrm{TM}}$ formulation.

\section{Notes and References}

1. J. J. Sanchez and S. A. Letts, Fusion Technol. 31, 491 (1997).

2. V. Malba et al., J. Vac. Sci. Tech. 15, 844 (1997).

\section{Producing NIF Mandrels Using the Microencapsu- lation/Decomposable Mandrel Technique}

As noted in the introductory section, the mandrel for the NIF capsule is critical since it sets the baseline sphericity of the capsule. The 2- to 3-mm-diam shells required for NIF targets ruled out production by the previously used solution drop tower technique that was used historically for 0.5-mm-diam Nova capsules. ${ }^{1}$ The decomposable mandrel technique, ${ }^{2}$ which is currently used to produce shells $<1 \mathrm{~mm}$ in diameter, ${ }^{3}$ has been adopted as the approach most likely to produce acceptable NIF mandrels. This process involves three steps. The first is the use of microencapsulation technology 4,5 to produce a very spherical poly $(\alpha-$-methylstyrene) (PoMS) shell. This shell is then overcoated with a uniform 5- to 10- $\mu \mathrm{m}$-thick layer of plasma polymer. In the final step this overcoated shell is heated to $300^{\circ} \mathrm{C}$, which causes the underlying P $\alpha \mathrm{MS}$ shell to depolymerize to gaseous products that diffuse away, leaving a thermally stable shell with the sphericity of the original PoMS shell. The last two steps in this process have been well developed;, 6 thus our focus has been on improving the initial microencapsulation process to produce PoMS shells that meet the rigid specifications required for the NIF capsule designs.

The microencapsulation process involves using a triple-orifice droplet generator to produce water droplets encapsulated by a fluorobenzene solution of P $\alpha \mathrm{MS}$ and supported in an aqueous bath. The bath typically contains a few wt $\%$ of polyvinyl alcohol (PVA), which serves to prevent agglomeration of the fluid droplets, and a small amount of inorganic salt to suppress water concentration in the oil phase, which results in vacuole defects in the final dry shell. ${ }^{8}$ As the fluorobenzene dissipates into the bath, a solid PoMS shell is formed, and the interior water phase can then be removed by air-drying. At the beginning of FY99, P $\alpha M S$ mandrels could be made at NIF size $(2-3 \mathrm{~mm})$, but the batches had both poor yield and shells typically with mode-2 (out-of-round) deformations in excess of $5 \mu \mathrm{m}$. Thus the work this year focused on improving both the batch yield and the shell out-of-round.

The batch yield was compromised by the necessity of continually tumbling the shells in a propeller-stirred water bath during curing to minimize gravitational deformation ${ }^{9}$ as well as to promote centering of the inner water droplet required for 


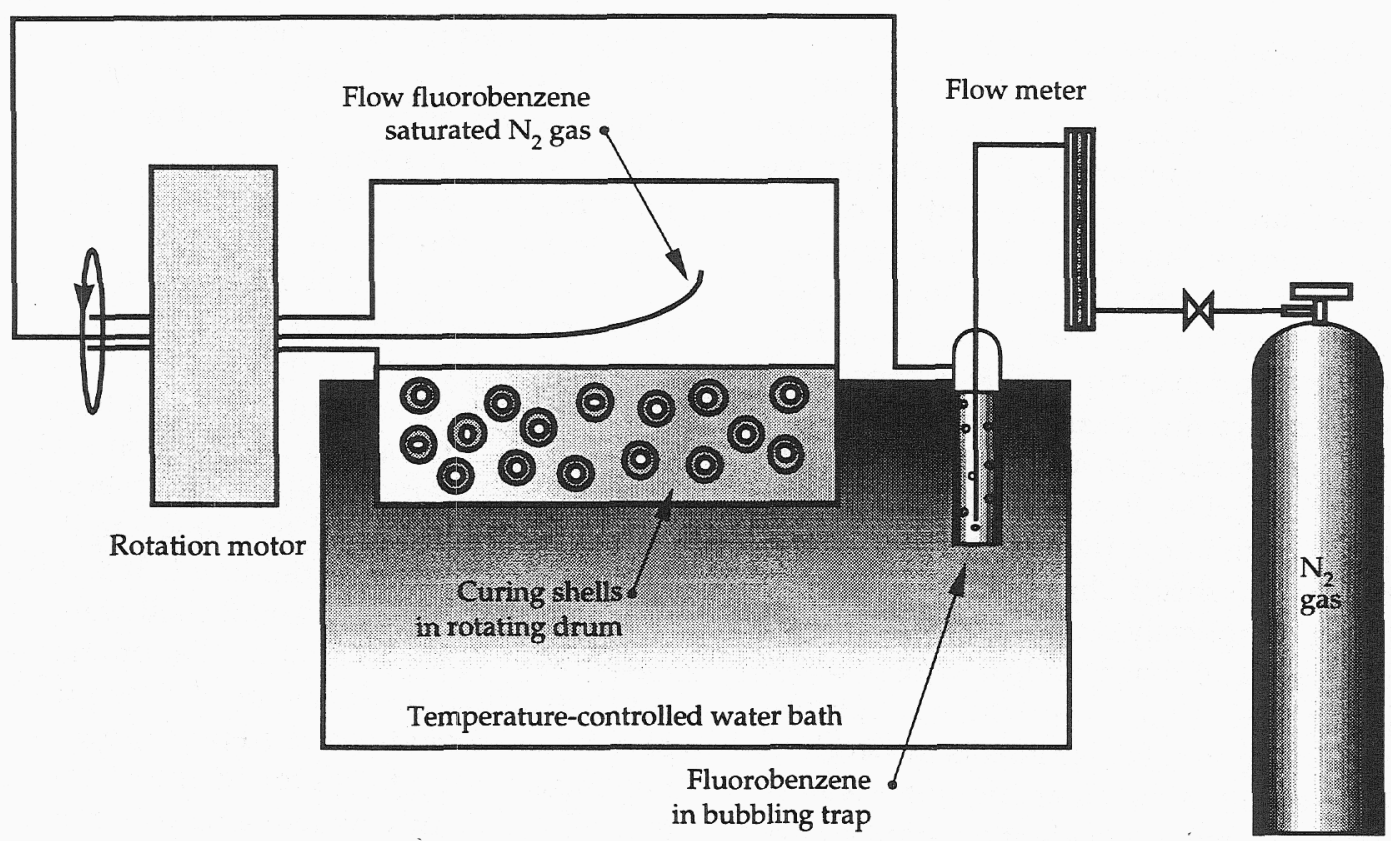

FIGURE 9. Schematic of rotating cylinder for curing compound drops. The horizontally rotating cylinder is almost halffilled with a PAA/water solution with viscosity $\sim 10 \mathrm{CP}$. The fluorobenzene is flushed out of the system by flowing $\mathrm{N}_{2}$ gas through the cylinder. The flushing rate is limited by partially saturating the $\mathrm{N}_{2}$ with fluorobenzene before it enters the cylinder. (40-00-1100-6110pb01)

uniform walls. ${ }^{10}$ This year we replaced the propeller-stirred bath with a horizontally rotating cylinder (Figure 9). ${ }^{11}$ This eliminated propeller collisions, hence increasing batch yield, and yet tumbled the shells so effectively that the wall thickness varied by only $\sim 1 \%$ (Figure 10 ).

The final shell mode 2 (out-of-round) is determined by the balance between the distorting forces (fluid shear and gravity) and the restoring force (interfacial tension). ${ }^{13}$ In a series of experiments aimed at understanding the time dependence of the cure (shell hardening) process, we measured the fluid shell deformation relaxation time as a function of cure time, which we related to polymer concentration and viscosity of the shell. The key conclusion of this work is that deformation relaxation is still rapid at times when the polymer concentration and viscosity is high enough to prevent core water decentering. ${ }^{14}$ We have also found that substituting polyacrylic acid (PAA) for PVA in the bath increased the interfacial tension by about a factor of $20 .{ }^{15}$ This resulted in mandrels whose out-of-round is routinely less than $1 \mu \mathrm{m}$ and thus well below the NIF low mode specification.

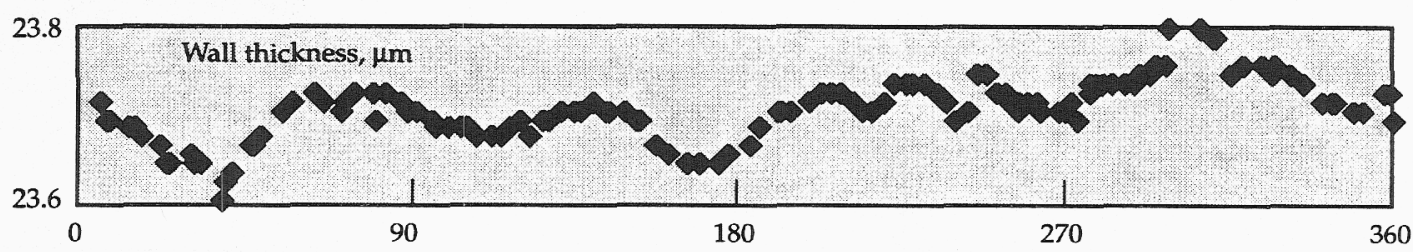

FIGURE 10. Wall thickness of a PaMS mandrel around an equator. The measurement was made with a General Atomicsdesigned wallmapper. ${ }^{12}$ The wallmapper focuses white light onto a $100-\mu \mathrm{m}$-diam spot on the shell and uses the oscillations in the reflection spectrum to calculate the wall thickness at that point. $\quad(40-00-1100-6111 \mathrm{pb} 01)$ 
Although the use of PAA greatly improved the mode- 2 symmetry of our PaMS shells, it had no significant effect on higher-mode asymmetry. In particular, we have found that a common feature of microencapsulated shells is unacceptably high power in the mode- 8 to 15 region of the capsule-surface power spectrum. This feature is often easily visible in capsule atomic force microscope (AFM) equatorial traces, appearing as a slight "wrinkling" of the surface. Our first suspicion was that the salt used in our curing baths was causing the shell to shrink slightly during cure due to the osmotically driven water flow out of the shell, and that this shrinkage in a stiffening shell might cause surface deformations. A series of experiments in which the level of salt in the inner and outer water phases were independently varied showed that we could control the degree of shrinkage of the shell during cure, and even cause it to swell slightly by having excess interior salt. ${ }^{16}$ However, these experiments also showed that the wrinkling was not directly related to this osmotic effect. Instead, they showed that some of the wrinkling was occurring during the drying process when the internal water is removed, well after the curing process is complete and the shell wall is solid.

The wrinkling may be related to a Marangoni instability within the curing shell wall that is driven by a PaMSconcentration-dependent interfacial tension. Rough calculations suggest that such an instability would produce convection cells that are roughly on the same length scale as the observed wrinkling. ${ }^{17}$ It is possible that this leads to a small shell-wall thickness or density variation that promotes a wall deformation during the drying process. We have modified shell processing to minimize driving forces for such convection cells, primarily by reducing concentration gradients in the curing wall by slowing down the curing process, and have made some progress in reducing the surface wrinkling in recent shell batches (Figure 11).
FiguRE 11. Power spectra of NIF-sized shells made in January (light) and in October (dark), 1999. The low modes and surface wrinkles have been reduced by changes in the mandrel production process. Vacuoles in the later shell, due in part to the elimination of the use of salt with PAA, have increased the high modes. (40-00-1100-6112pb01)

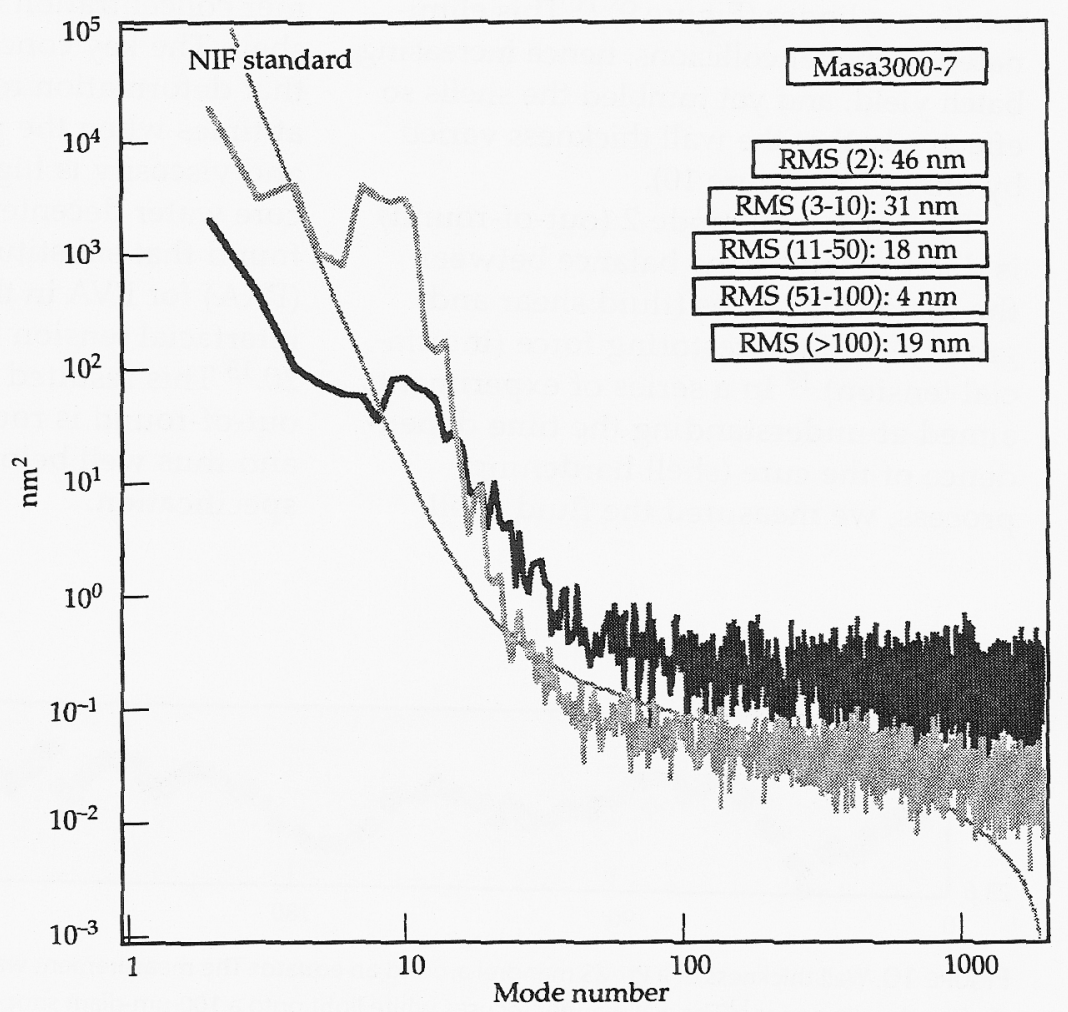




\section{Notes and References}

1. R. Cook, Mat. Res. Soc. Symp. Proc. 372, 101 (1995).

2. S. A. Letts et al., Mat. Res. Soc. Symp. Proc. 372, 125 (1995); S. A. Letts et al., Fusion Technol. 28, 1797 (1995).

3. B. W. McQuillan et al., Fusion Technol. 31, 381 (1997).

4. M. Takagi et al., J. Vac. Sci. Technol. A, 9, 2145 (1991).

5. B. W. McQuillan and A. Greenwood, Fusion Technol. 35, 194 (1999).

6. Ibid.; S. A. Letts et al., Mat. Res. Soc. Symp. Proc. 372, 125 (1995); S. A. Letts et al., Fusion Technol. 28, 1797 (1995).

7. Ibid.; B. W. McQuillan et al., Fusion Technol. 31, 381 (1997).

8. B. W. McQuillan et al., Fusion Technol. 35, 198 (1999).

9. R. C. Cook et al., J. Moscow Phys. Soc. 8, 221 (1998).

10. T. Norimatsu et al., Fusion Technol. 35, 147 (1999).

11. M. Takagi et al., Fusion Technol. (2000), in press.

12. R. B. Stephens et al., Fusion Technol. (2000), in press.

13. Ibid.; R. C. Cook et al., J. Moscow Phys. Soc. 8, 221 (1998).

14. M. Takagi et al., Fusion Technol. (2000), in press.

15. Tbid.; M. Takagi et al., Fusion Technol. (2000), in press.

16. M. Takagi et al., Fusion Technol. (2000), in press.

17. B. McQuillan et al., "Marangoni ....," presented at the $13^{\text {th }}$ Target Fabrication Meeting, Catalina Island, Calif., Nov. 8-11, 1999.

\section{Extending Layering} Techniques to Thinner Layers $(80 \mu \mathrm{m})$ and Lower Temperatures Required for NIF

\section{IR-Heating}

Infrared heating is a technique used to grow uniform DH or DD fuel layers or to enhance the layering rate/uniformity of DT fuel layers in plastic capsules. Early this year we converted from a continuous wave (CW) F-center mid-IR laser to a pulsed optic parametric oscillator laser (OPO) to allow experiments with increased bulk heating. We find that the coupling of IR power into bulk heat is about the same for the pulsed source ( $10 \mathrm{~ns}$ at $20 \mathrm{kHz}$ ) vs the CW source, suggesting the rotational-vibrational relaxation to phonons and that our original lower-bound estimates for this process are likely accurate.
Experiments this year addressed two critical questions: (1) How does He gas inside the vapor affect layer uniformity and layering rate? (2) Can a NIF-scale target be cooled rapidly enough to the designpoint temperature so that the layer does not deform before the final design-point gas density is reached? Helium gas may need to be added to NIF ignition or OMEGA cryogenic implosion targets for diagnostic purposes. Helium gas is also present in DT-filled capsules since it is the decay product of tritium. Thus it is important to determine how surface roughness and redistribution time constants change with $\mathrm{He}$ gas pressure. Experiments were performed on HD layers contained inside an $~ 30$ - $\mu$ m-thick 1-mm-o.d., CD plasma polymer shell set inside a 1-in.-i.d. goldcoated aluminum IR integrating sphere. Time-constant measurements were made at about $1 \mathrm{~K}$ below the triple-point temperature $\left(T_{\mathrm{tp}}\right)$ and at a laser power of $\sim 9 \mathrm{~mW}$. The time constant at this power with no He added was $17 \mathrm{~min}$, indicating roughly twice the bulk heating rate compared with beta-decay heating. Layers for surfaceroughness experiments were performed by standard slow-cool layering techniques. With this setup, we find that both time constants and surface roughness (modes 2-100) are unchanged over the zeropressure He case between 0 and 400 Torr of He pressure added to the shell cavity. Above 600 Torr, there is an observed increase in both time constant and surface roughness. Note that 640 Torr of $\mathrm{He}$ in a shell at $18 \mathrm{~K}$, with fuel layer aspect ratio of 0.1 , is equivalent to a $\mathrm{He}-3$ buildup from beta decay for a period of about 200 days ( $\sim 3.2$ Torr/day). This is much longer than the expected time between DT fill and NIF target shots since simulations suggest target performance begins to degrade after only a few days buildup of $\mathrm{He}$. At times of order a week, based upon these recent data, we would expect very little effect from $\mathrm{He}-3$ on time constants or surface roughness.

Similar to the DT experiments, the goal of recent IR layering experiments is to generate uniform fuel layers at temperatures $\sim 1.5 \mathrm{~K}$ below $T_{\mathrm{tp}}$. In contrast to the DT experiments, the focus here is to rapidly 
freeze the layers so that the equilibrium vapor pressure is reduced before the solid deforms. One main question is, can we lower the temperature faster than the layer roughens, i.e., $\tau_{\text {cooling }}<\tau_{\text {roughening }}$ ? If so, there should be a small window in time where the ice layer is relatively smooth at $\sim 1.5 \mathrm{~K}$ below the triple point.

In these recent experiments, rapid cooling was achieved by turning off the IR illumination after a layer formed at $T_{\mathrm{tp}}$. The time constant for cooling the ice is given by $\tau_{\text {cooling }}=C / K$, where $C$ is the heat capacitance of the ice and shell, and $K$ is the thermal conductance of the surrounding He gas. Using a 1-mm-o.d. shell with a 100 - $\mu$ m-thick ice layer, one can estimate that $\tau_{\text {cooling }}=1 / 2 \mathrm{sec}$. This requires collecting images an order of magnitude faster than our other experiments. To acquire images more rapidly, we reduced the image resolution. Low-resolution shadowgraph and interferometric images were acquired at 30 frames per second, and high-resolution shadowgraph images were captured about every 14 seconds during these rapid-freeze experiments.

We are still developing our data acquisition and analysis techniques for these lowresolution images. Below are two figures from our preliminary data analysis. In Figure 12 we have plotted the temperature drop of the capsule along with a set of lowresolution images. The arrows indicate the time at which the images were acquired. Figure 13 shows the power spectra from the high-resolution images for a layer just prior to turning off the IR and then 5 seconds later. These data reveal the possibility that we can reach the design-point temperature and preserve the surface finish by rapid cooling; however, the surface-roughness data analysis, rapid-cooling techniques, and determination of gas fill pressure need significantly more work.

\section{DT Layering Experiments}

The Safety Note, written and operational safety procedures (OSP) for DT layering experiments, was completed in the first quarter, and DT layering experiments resumed. The primary observation is that using the center of the bright band (described below) as an indicator of the actual ice-gas interface, NIF quality DT layers can be formed from the $T_{\mathrm{tp}}$ to $\sim 0.5 \mathrm{~K}$ below this temperature. Furthermore the surface-roughness power spectrum falls more rapidly than previous spectra determined from cylinder experiments. Below $T_{\mathrm{tp}}-0.5 \mathrm{~K}$, the DT surface roughness begins to degrade. Thus the current goal for the DT layering experiments is to
FIGURE 12. Plot of the drop in capsule temperature vs time along with video images acquired at those corresponding points in time. (40-00-1100-6113pb01)

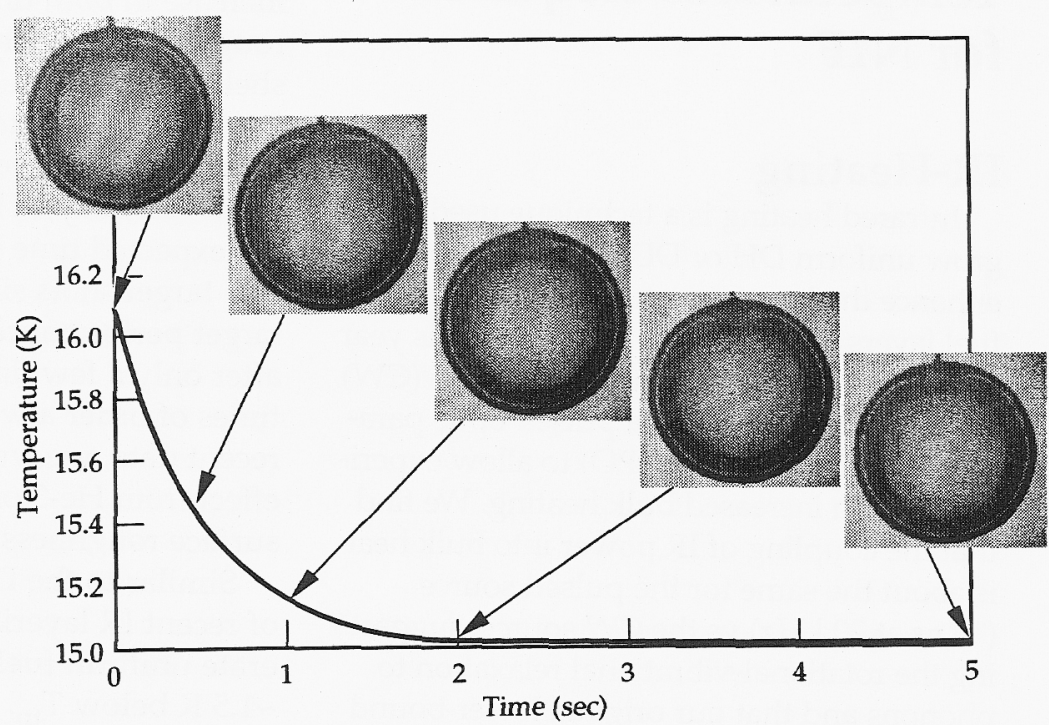




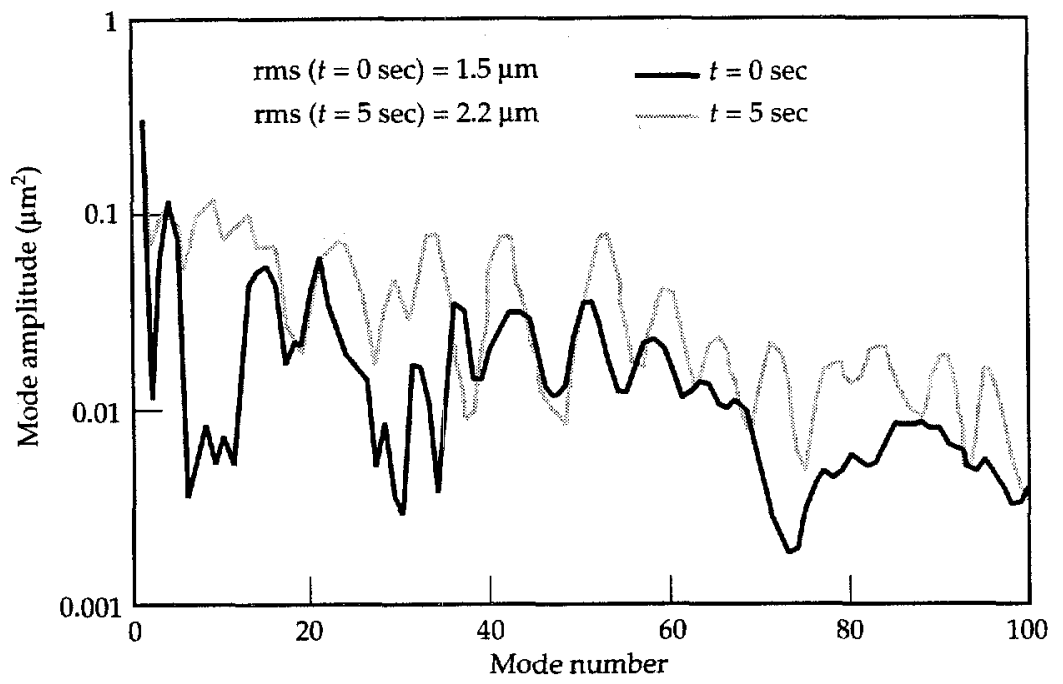

FIGURE 13. Power spectra showing the degradation of the layer after the IR is turned off. (40-00-1100-6114pb01)

achieve a smooth ice layer at $T_{t p}-1.5 \mathrm{~K}=$ $18.3 \mathrm{~K}$, which is the NIF design point. The experimental hypothesis is that DT will creep at a slow rate under stress. Data from experiments indicates that this is true for $D_{2}$. If we form a smooth layer near the DT triple point and cool slowly enough to $18.3 \mathrm{~K}$, the layer should relieve internal strains by creeping and not cracking. Hopefully, the layer would still be smooth. We have conducted experiments with a cooling rate as slow as $0.25 \mathrm{mK} / \mathrm{min}$. Preliminary analysis indicates that the layer begins to degrade at temperatures on the order of $19.3 \mathrm{~K}$ even at these slow cool rates. The expected limit to how slowly a layer can be cooled to the design-point temperature, as described above, is set by the He buildup in the central cavity. There is also the formation of $\mathrm{He}$ bubbles, which grow in layers at temperatures below $T_{\mathrm{t}}$. Precisely how much He diffuses out of the bulk solid and into the vapor region during and after a DT layer is formed is a focus of ongoing work.

To grow and cool layers at extremely slow cool rates, we needed to improve our capsule/fill-tube technology to make more robust assemblies. Previous shell/ fill-tube assemblies lasted on the order of only a few weeks. This improved target assembly included a 2-mm-o.d. plasma polymer capsule with a 30- $\mu \mathrm{m}$ fused silica fill tube. An additional advantage to the current shell assembly technique is that the wall uniformity is much improved over previous designs. This allows us to measure the effects of microwave heating on DT layers in shells at $T_{\text {des }}$. We find that for an E field of about $400 \mathrm{~V} / \mathrm{cm}$, there is a significant reduction in mid- to high-mode roughness at the design-point temperature, suggesting that this technique may produce smooth targets at $18.3 \mathrm{~K}$. There is, however, a very large $P_{2}$-like defect due to variations of the E-field power density in the current cavity design. Initial estimates suggest that the E-field uniformity needs to be better than $\sim 1 \%$ to reduce such asymmetries. Future work on Joule heating will be to define plausible NIF fielding scenarios and if successful, improve the spherical cavity E-field uniformity to test our hypothesis.

\section{Layer and Opaque Shells Characterization}

The workhorse for DT layer characterization has been edge detection of shadowgraph images. Early this year, results from simulating the effects of isolated defects on the shadowgraph images indicated that the center of the bright band ( $\mathrm{a}$ bright circle in the shadowgraph image caused by internal reflection off of the ice gas interface) is a better indicator of gas-solid interface position in spheres than the band edge. Thus we modified and tested the 
analysis code to fit the intensity profile of the bright band to a Gaussian plus a 2 nd order polynomial. The modified code takes the center of the Gaussian as the indicator of the DT gas-solid interface. This analysis is still preliminary but yields a significantly lower surface roughness for high-order modes than the edge detection scheme.

Our initial shadowgraph "isolated defect study" used a commercial raytracing code to determine the effects on the bright band due to different (1) sizes of defects, (2) ice layer thicknesses, (3) wall thicknesses, and (4) light source configurations. Because of limitations to the type of perturbations allowed by most commercial ray-tracing packages, we wrote and tested an exact numerical ray-trace simulation code specifically for light transfer through a transparent cryogenic shell. This code was used to test our ability to extract icesurface asphericity information from backlit shadowgraphy by simulating the shadowgraphs resulting from ice surfaces with known multimode imperfections. We generated simulated shadowgraphs, initially treating the ice surface as a sum of rotationally symmetric Legendre modes. The ice-surface power spectra derived from the shadowgraph analysis of these simulated images were then compared against the known ice-surface power spectra, under various conditions including diffuse and collimated backlighting. An example of power spectra from a model surface and a "detected" surface are shown in Figure 14. In general, we find that the Gaussian fit best reproduces the surface profile with the illumination geometry used in the experiments. The brightband analysis reproduces the actual ice-surface power spectrum rather well, with rms roughness deviations of less than $15 \%$, for mode numbers from 1 to 40 and perhaps as high as 80 . The effects of twodimensional (spherical-harmonic) mode structure was also started.

In addition to shadowgraphy, we are developing two interferometric techniques to enhance our capability to characterize shells inside hohlraums. The first interferometer we describe is a phase-stepping interferometer, which was installed on the IR layering experiments. Three analysis methods give information about the ice roughness: (1) interferometric information in the bright band, which is also analyzed by shadowgraphy, (2) Fourier analysis of $1-\mathrm{d}$ rings selected from the phase map (collaboration with University of Rochester's Laboratory for Laser Energetics [LLE]), and (3) analysis of the whole interferogram. We
FIGURE 14. An example of power spectra from a model surface and a "detected" surface. (40-00-1100-6115pb01)

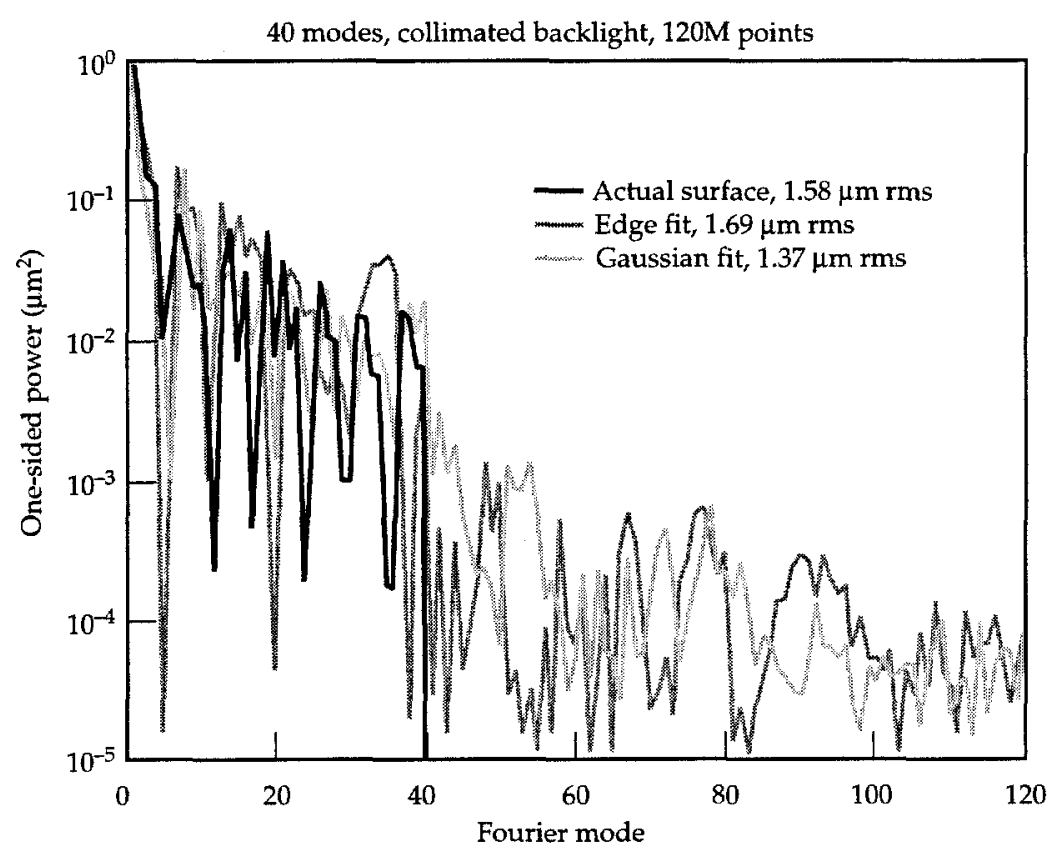


have successfully collected data from the interferometer using phase stepping. We have also been able to use Pat McKenty's (LLE) code to analyze our interferograms, though results are currently limited by poor resolution from fringe jitter.

A Fourier-transform reflection interferometer to diagnose DT ice surface roughness in a transparent shell was also constructed and is currently being tested with the beta-layering experiments. This diagnostic works by bouncing broadband light off the multilayered shell $(\mathrm{CH}+\mathrm{DT}$ layers) and measuring the Young's fringes of the reflected light. The fringe spacing is proportional to layer thickness so that by using an imaging spectrograph, we obtain a film thickness vs position measurements with nanometer accuracy in thickness. In addition to the extreme sensitivity to layer thickness for high-frequency modes, we also determine the very-lowest-order modes along the axis of the hohlraum because this technique works coaxially in reflection. The development of this technique is a major step in ignition target characterization.

In an effort to build a diagnostic to characterize DT layers in opaque shells, we have looked into acoustic techniques that have promise of in situ characterization on the NIF. Using a femtosecond optical pump-probe experiment, we have demonstrated detection of echoes from laser-generated ultrasound waves propagated into thin film (up to $2000 \AA$ ) targets including Be and other metals. Results indicate an acceptable signal/noise; however, the echo signature is mixed in a complicated way with the reflectometry signature. With these results in hand, this technique appears to be extremely complex to implement on cryogenic capsules. Preliminary studies of alternatives to this time-domain technique have also begun. A resonance ultrasound spectroscopy method using either modulated electrostatic or microwave driving forces has been studied theoretically, and the numbers indicate that a proof-of-principle experiment is worthwhile. Design and implementation of an electrostatic experiment are awaiting analysis of the coupling between Be and DT ice modes as well as an estimate of the frequency resolution of the detection.

\section{Symmetry and Control of $P_{1}$ and $P_{2}$ in NIF Prototype Cryogenic Hohlraum}

This effort is focused on developing the technology to field cryogenic hohlraums with DT-filled capsules. The idea is to thermally shim the hohlraum to produce a uniform spherically symmetric heat flow away from the capsule wall. The major thrusts of this effort this year are to model the cryogenic hohlraum environment to optimize the design of the thermal symmetrizer, fabricate cryogenic hohlraums, and compare experimental symmetry control experiments with modeling.

The present schemes for thermally shimming the hohlraum consist of using heaters on the midsection of the hohlraum and on the sapphire support rods for controlling the axial hohlraum temperature. In addition to this, the sapphire support rods are connected to the hohlraum through an equalizer, which is designed to create a nearly uniform azimuthal temperature. The equalizer must be soldered onto the hohlraum at four points; if the thermal conductivity through these solder points varies significantly from point to point, large azimuthal temperature asymmetries may develop. We developed an infrared thermal emission technique to diagnose the half-hohlraum conduction uniformity at room temperature before full assembly of the hohlraum. For our first hohlraum assembly, this diagnostic revealed a $P_{1}$ asymmetry of about $0.2 \mathrm{~K}$, with the warmer side opposite to the cooling rod. This room temperature measurement can be scaled to obtain the expected temperature variation at cryogenic temperatures by accounting for the factor of 10 increase in thermal conductivity and factor of 4 to 5 decrease in the thermal input power. This gives an overall expected azimuthal $P_{1}$ variation at cryogenic temperatures of about 4 to $5 \mathrm{mK}$, which is thought to be acceptable.

The engineering Safety Note was written, the gas handling and the vacuum systems were pressure tested, and the Hohlraum Test System OSP was finished. 
Finally, with axial symmetry at hand and safety documentation in place, we are ready to begin thermal shimming experiments.

\section{Modeling}

Previous modeling has culminated in the current hohlraum design discussed above. Current modeling attention is focused on understanding and controlling the effects of convection. We created a fourfold symmetric model of the hohlraum to calculate convective heat transfer effects on the DT layer. Preliminary indications from this model are that convective heat transfer produces a thermal asymmetry about 100 times larger than acceptable. Some of this asymmetry can be opposed by placing a linear gradient across the hohlraum axis. In addition, a thin film parallel to the capsule support film and tangent to the top of the capsule also significantly reduces this convective-induced asymmetry. The combined effects of a linear gradient and single film reduce the asymmetry, but the thermal asymmetry is still too large by a factor of 5. We are modeling the effects of additional films to reduce these asymmetries to acceptable levels. 


\subsection{NIF LASER DEVELOPMENT}

$\mathrm{T}$ he fiscal year 1999 laser technology development activities in support of the NIF design and deployment were centered around five themes: (1) main amplifier cleanliness, (2) preamplifier prototype testing, (3) development of low-cost capacitor technology, (4) development of $3 \omega$ fiber technology for power diagnostics, and (5) completion of the deformable mirror prototype qualification effort.

\section{Contamination and Amplifier Slab Damage}

For several years it has been observed that various levels of damage occur to laser amplifier slabs during normal laser operations. The damage phenomenon has nearly always been associated with the introduction of extrinsic (external) particulate contamination such as the inadvertent exposure of gaskets to laser and/or flashlamp light resulting in the generation of massive amounts of burned elastomer particles that form an aerosol and eventually settle everywhere inside the laser cavity. A somewhat related intrinsic (internal) damage has been associated with the inclusion of small platinum particles within the laser glass. These are opaque to the laser light, heat rapidly under flashlamp and laser light, and literally explode within the laser glass. This intrinsic damage problem has been eliminated by changes to the glass fabrication process. Elimination of the extrinsic damage problem will require (1) a more fundamental understanding of the damage generation mechanism, (2) a more precise understanding of the types of contaminants that lead to slab damage, (3) identification of all sources of the aerosol particles, (4) development of cleaning techniques to provide sufficient initial cleanliness, and (5) the selection of lowoutgassing and high-damage-threshold structural materials that will minimize the formation of aerosols in the presence of laser and flashlamp light.

\section{Description of Flashlamp- Induced Damage}

Maintenance operations on Argus, Shiva, Nova, and Beamlet laser facilities have observed various levels of damage to the laser amplifier slabs. As early as Argus, it was also observed that this damage occurred without the need for laser propagation; that is, damage was observed after only flashlamp exposure. Figure 1 shows a highly magnified image of a slab damage site induced from a single flashlamp firing.

Small contaminant particles resting on or near a laser slab surface become small UV-emitting fireballs when illuminated with flashlamp light and quickly come into radiative equilibrium with the $\sim 1-\mathrm{eV}$ flashlamp black-body temperature.

Because of the high thermal expansion coefficient and low tensile strength of the laser glass, it crazes, and shallow surface 


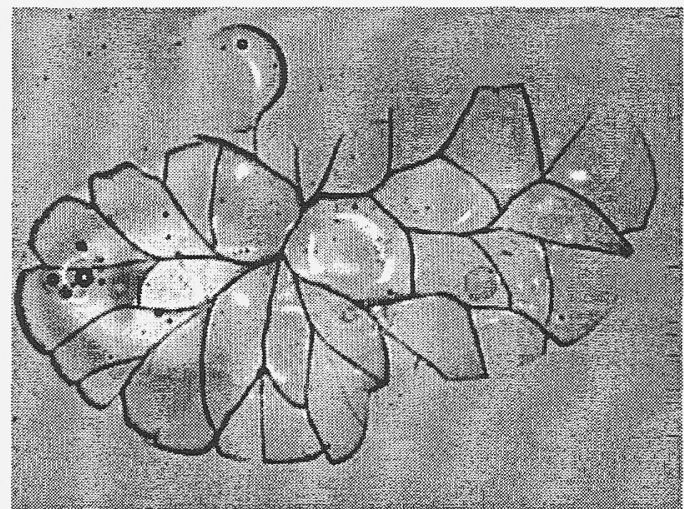

FIGURE 1. Microscopic image of laser slab damage $(\approx 500 \mu \mathrm{m}$ in length) resulting from purposeful contamination of borosilicate glass by low-density polyethylene and then exposure to a single flashlamp firing. The absorbing contaminant particles are rapidly heated by the flashlamp light to the plasma temperature of $10,000^{\circ} \mathrm{K} . \quad(40-00-1100-6116 \mathrm{pb} 01)$

cracks quickly form on the surface. The mechanical properties of laser glass are such that glasses with a high thermal expansion coefficient, low thermal conductivity, and low tensile and fracture strength will be most susceptible to flash- burn crazing (fracturing) when exposed to intense flashlamp light. The thermal shock parameter is a nondimensional parameter that provides an indication of the relative sensitivity of materials to crazing, and the phosphate laser glass, being produced for the NIF, is particularly sensitive to this form of fracturing.

Menapace $^{1}$ found that the size of the damage site exceeded the size of the contaminant by a factor of 7.7 as shown in Figure 2. An opaque particle will intercept and be heated by the flashlamp fluence of $10 \mathrm{~J} / \mathrm{cm}^{2}$. Particles with no effective thermal sink must rise in temperature until they are able to radiate their energy. The fireball so produced illuminates the nearby glass surface with a spectrum, including UV wavelengths, to which the glass is highly absorbing. At some distance away from the fireball on the glass surface, the deposited UV energy falls below the level sufficient to exceed the critical stress for fracturing the material. Since the lamp power coupling to the opaque particle, as well as its reemission, must scale as the particle area, and the surface burn area also scales
FIGURE 2. Results from experiments involving the purposeful contamination of laser glass surfaces and the subsequent irradiation of the surfaces with a oneto three-flashlamp exposure. The damage after irradiation is typically 7.7 times larger than that caused by the initial contaminant. (40-00-1100-6147pb01)
Flashlamp-induced damage to phosphate and borosilicate glasses from surface contaminants ( 1 to 3 shots)

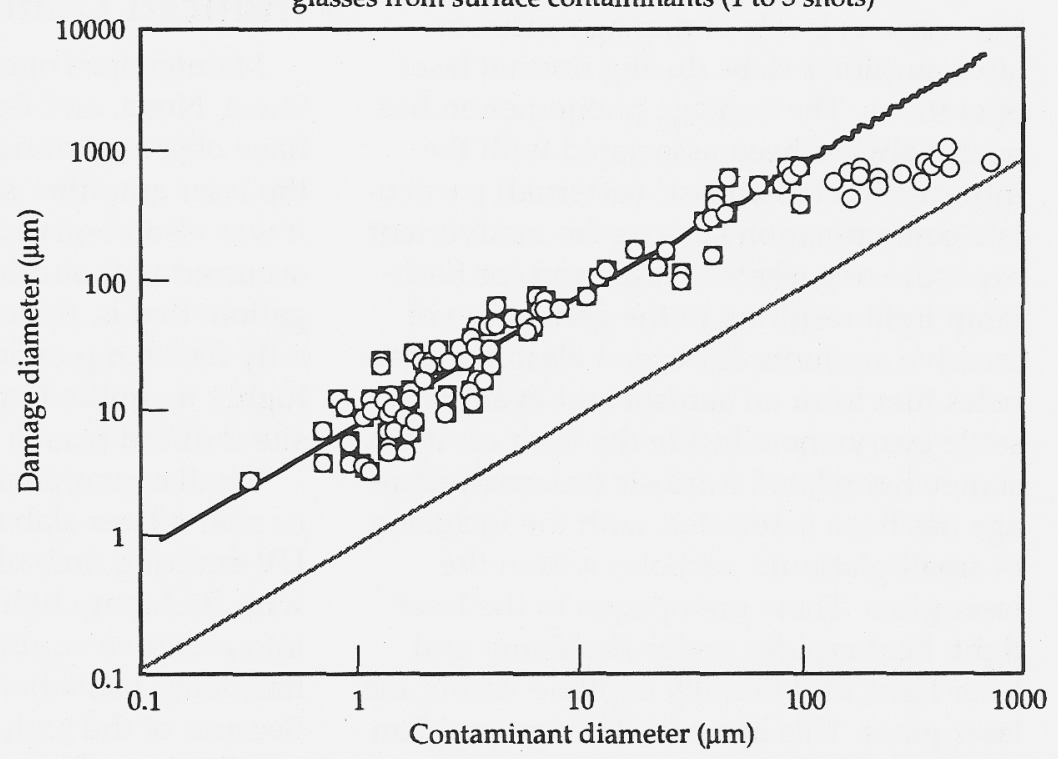


linearly with reemission power, the flashburn diameter should scale linearly with particle diameter. Consequently, the rapid heating of an opaque contaminant results in local heating of the glass surface, which can be relieved by the large-scale but shallow fracturing of the glass. Shallowly buried laser-heated particles, by contrast, lead to pits approximately one-third as deep as they are across, where the volume of material removed scales with the absorbed fluence. The most profound result of this analysis is that the resulting size of the fractured area scales linearly with and can be significantly larger than the size of the contaminant that initiated the damage.

\section{Contamination and Maximum Damage Size Criteria}

Initial optical and structural surface cleanliness levels are derived from the calculated scatter loss resulting from the size distribution of various cleanliness levels. Cleanliness levels are comprehensively defined in MIL-STD-1246C. ${ }^{2}$

The area obscured by any given size distribution (or level of cleanliness) is easily computed, and a Level-100 optical surface will obscure (or scatter out of the beamline) only $1 \times 10^{-6}$ of the incident light. Therefore, optical surfaces could be allowed to become contaminated (or damaged) to roughly Level 180 before the distribution of contaminants would result in a maximum desired scatter loss of $2.5 x$ $10^{-5}$. However, it is impractical to allow this level of contaminants to accumulate as Level 180 will statistically allow a single 234- $\mu \mathrm{m}$-diameter particle per slab surface, and each such particle may result in a $1,800-\mu \mathrm{m}$-diameter damage site $(234 \mu \mathrm{m} \times$ 7.7). This damage size closely approaches the maximum size allowable (2-mm) to prevent possible damage to spatial filter lenses. Therefore, both initial and operational optical cleanliness levels must be maintained at least one order of magnitude cleaner than defined by Level 202, which statistically leads to a single $2-\mathrm{mm}$ damage site per slab surface. Level 202 divided by 10 yields a maximum optical surface cleanliness of Level 114.

\section{Role of Aerosols in Flashlamp-Induced Damage}

Stowers ${ }^{3}$ first observed the creation of an aerosol in a Shiva laser amplifier circa 1980; this was reconfirmed on Beamlet in 1997. The background aerosol level in an unfired amplifier is Class 1 to 10 ( 1 to 10 particles $\left./ \mathrm{ft}^{3}>0.5 \mu \mathrm{m}\right) .{ }^{4}$ Immediately after firing, this aerosol level rapidly jumps to Class 100,000 to $1,000,000$. These aerosol levels are not sufficiently dense to be easily seen with the naked eye, but they are typical of outdoor aerosol levels in Livermore, California. Aerosols that are not flushed away will linger within an amplifier and, due to electrostatic charges and gravitational effects, will deposit onto laser slab surfaces.

Damage to laser slabs can occur on subsequent shots when settled aerosol particles absorb flashlamp light and rapidly heat. The heated particles thermally stress the laser glass and result in local cracking or crazing.

The aerosols that have been observed in amplifiers are believed to be generated when flashlamp light irradiates organic films, a.k.a. nonvolatile residue (NVR) on structural surfaces. The flashlamp light fluence of $10 \mathrm{~J} / \mathrm{cm}^{2}$ in $370 \mu$ is sufficient to pyrolyze and decompose these organic films on structural surfaces.

The composition and morphology of aerosol particles collected during tests in the Amplifier Module Prototype Laboratory (AMPLAB) were ascertained by sampling the aerosol with a cascade impactor. Figure 3 shows the particles that are composed of many 10- to $20-\mathrm{nm}$ nodules of nearly pure carbon. It is believed that the smaller particles form during the time the flashlamps illuminate, whereas the 3- $\mu \mathrm{m}$ particle clusters form due to the rapid collision of these smaller particles and agglomerate shortly after the flashlamps extinguish. 
FIGURE 3. High-magnification scanning electron microscopy (SEM) photograph of aerosol particles from AMPLAB experiments. The typically $3-\mu \mathrm{m}$ particles are composed of 10- to 20-nm nodules that are made of nearly pure carbon, indicative of decomposed organic matter.

(40-00-1100-6148pb01)

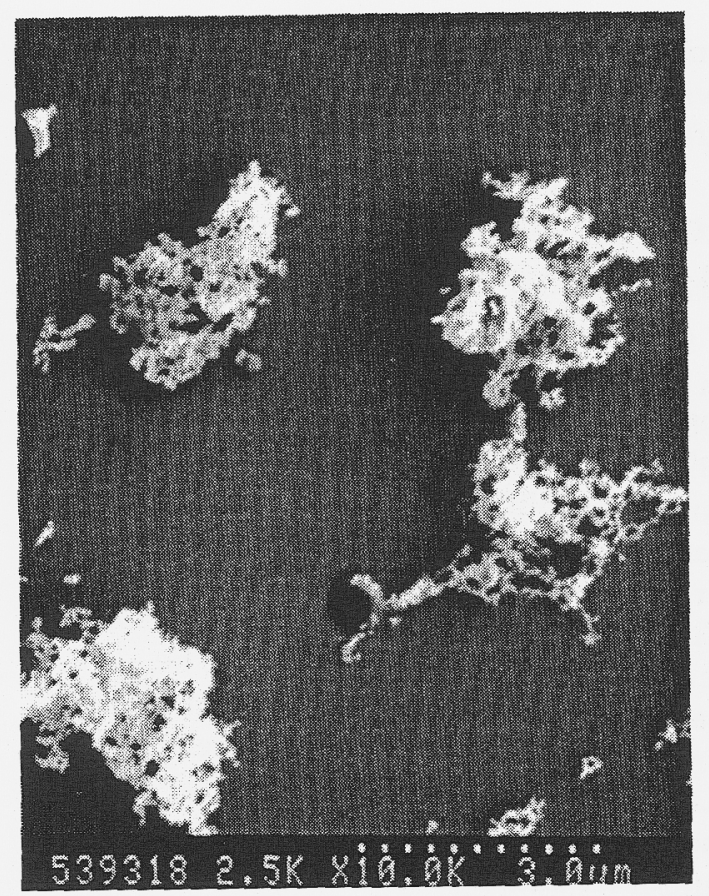

\section{Consequences of Slab Damage and Damage Size Distribution}

Widmayer and Renard ${ }^{5}$ used a sophisticated modeling code to predict the peak intensity on spatial filter input lenses based on small obscured spots at various locations on laser slabs. Results shown in Figure 4 indicate that defects as large as 1 -mm result in beam modulation (ratio of peak to mean intensity) of 1.75 , where this value is a factor of 2.7 below the laser damage threshold of the spatial filter lens. Widmayer et al. further conclude that the detection of a single opaque contaminant or damage site on a slab surface of $2,000-\mu \mathrm{m}$ (2-mm) diameter represents a safe removal and refurbishment criterion. ${ }^{6}$

Historically, slab damage has been recorded using damage maps as shown in

FIGURE 4. Predicted relative intensity on the cavity spatial filter (CSF) input lens due to small opaque defects of various sizes on the slabs preceding the spatial filter input lens. The presence of even a 7 -mm-size defect is safe and will not endanger the safety or the integrity of the CSF input lens. (40-00-1100-6149pb01)

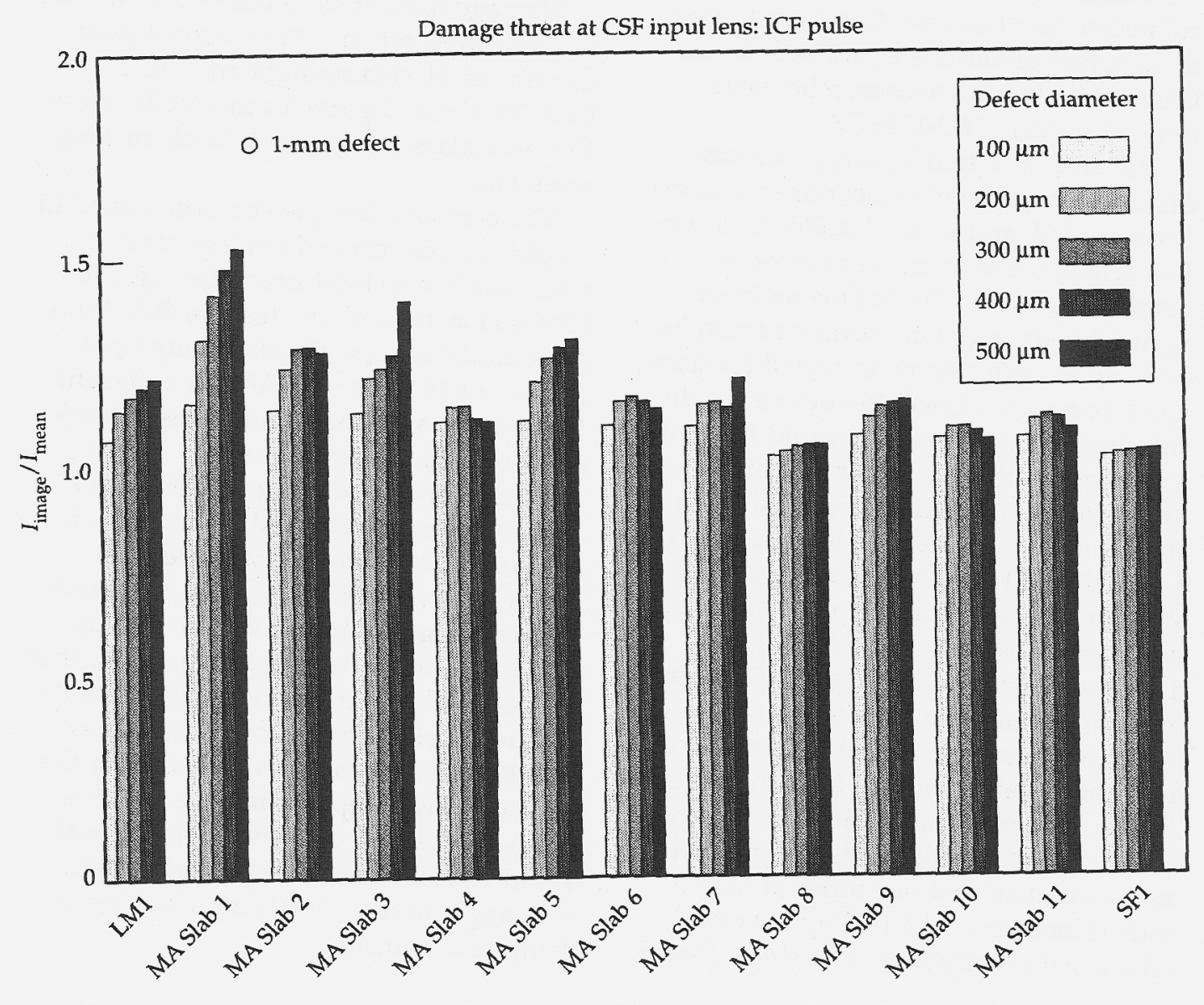


Figure 5. Each damage location is marked by a spot, where the spot size (not to scale) is indicative of the obscuration size. A histogram of these damage sites vs damage site diameter can be generated by normalizing the damage sites as obscurations $/ \mathrm{ft}^{2}$ and integrating the distribution to obtain a cumulative size distribution as shown in Figure 6.

Figure 7 shows cumulative damage curves for slabs removed from Nova when it was disassembled in 1999. The damage concentrations observed on Nova are typically 200-400 obscurations $/ \mathrm{ft}^{2} \geq 100 \mu \mathrm{m}$ and nearly $10 \times$ more frequent than damage observed on AMPLAB. The curves also demonstrate that many of the slabs had damage exceeding 1-2 $\mathrm{mm}$ in size.

Figure 8 shows the cumulative size distribution of laser slabs removed from Beamlet after 1,500 shots. The damage concentrations observed on Beamlet are typically $40-50$ obscurations $/ \mathrm{ft}^{2} \geq 100 \mu \mathrm{m}$. The majority of the laser slabs also had damage sites exceeding $1 \mathrm{~mm}$ in size.

Figure 9 shows the cumulative size distribution of one of the laser slabs tested during the AMPLAB experiments. The damage concentrations observed on AMPLAB are typically 10 obscurations/ $\mathrm{ft}^{2} \geq 100 \mu \mathrm{m}$. Damage in AMPLAB experiments was observed to increase slowly with the number of shots over the range from 220 to 750 shots. However, the slabs in these tests also had an unusually high level of initial surface damage.

In general, all Beamlet slabs had less damage than those removed from Nova and more damage than the slabs removed from AMPLAB. It is generally believed that the amount of damage is a function of the number of flashlamp shots, and Nova had substantially more shots than either Beamlet or AMPLAB. However, controlled experiments to demonstrate the growth of damage as a function of the number of flashlamp shots will not be done until FY2000. These controlled experiments are necessary to determine the expected replacement rate of the laser slabs.

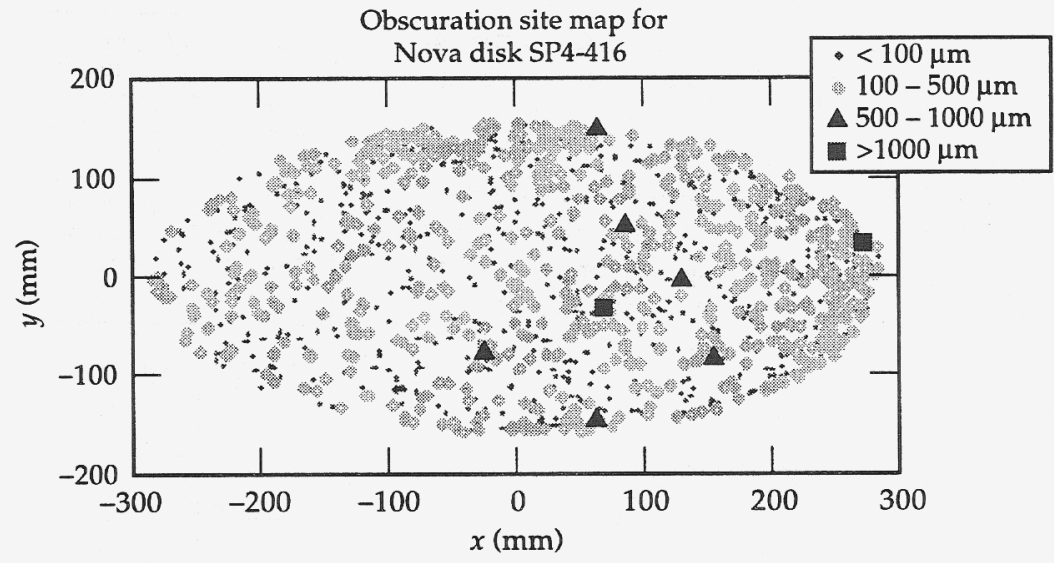

FIGURE 5. Obscuration site map for a typical Nova $31.5-\mathrm{cm}$ disk showing the location of the obscurations and their relative sizes. (40-00-1100-6150pb01)

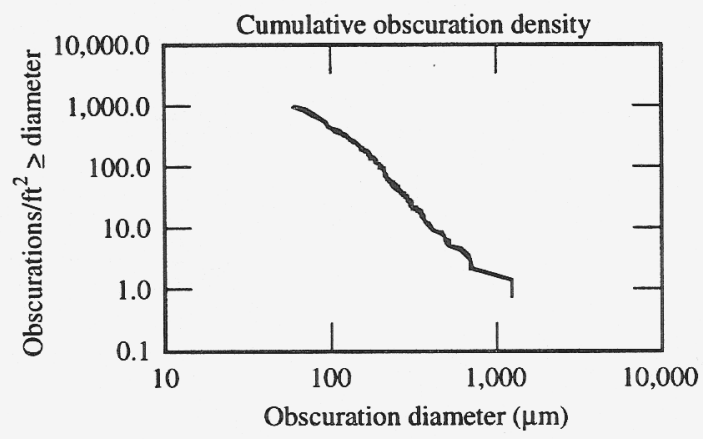

FIGURE 6. Cumulative obscuration density plot of Nova disk SP4-416 showing cumulative obscuration density vs diameter. (40-00-1100-6151 pb01)

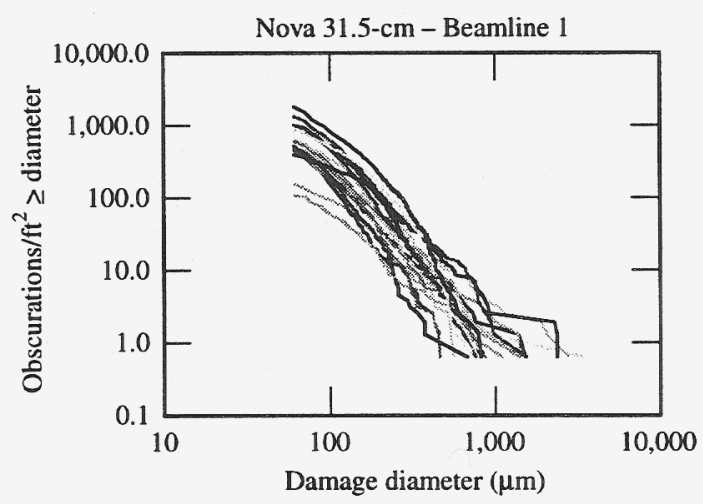

FIGURE 7. Cumulative obscuration density for Nova amplifiers on Beamline 1. The concentration at $\geq 100 \mu \mathrm{m}$ is approximately $200-400$ obscurations/ $\mathrm{ft}^{2}$. (40-00-1100-6152pb01) 
FIGURE 8. Cumulative size distribution measured on slabs removed from Beamlet when it was disassembled. The concentration at $\geq 100 \mu \mathrm{m}$ is approximately 40-50 obscurations/ $/ \mathrm{t}^{2}$. (40-00-1100-6153pb01)

\begin{tabular}{|lll|}
\hline$\rightarrow$ S/N 01 Input & S/N 06 Output & S/N 11 Input \\
S/N 01 Output & S/N 07 Input & S/N 11 Output \\
S/N 02 Input & S/N 07 Output & S/N 14 Input \\
S/N 02 Output & S/N 08 Input & S/N 14 Output \\
$\rightarrow$ S/N 03 Input & S/N 08 Output & S/N 16 Input \\
$\rightarrow$ S/N 03 Output & S/N 09 Input & S/N 16 Output \\
$\square$ S/N 05 Input & S/N 09 Output & S/N 17 Input \\
- S/N 05 Output & S/N 10 Input & S/N 17 Output \\
- S/N 06 Input & & S/N 10 Output \\
\end{tabular}

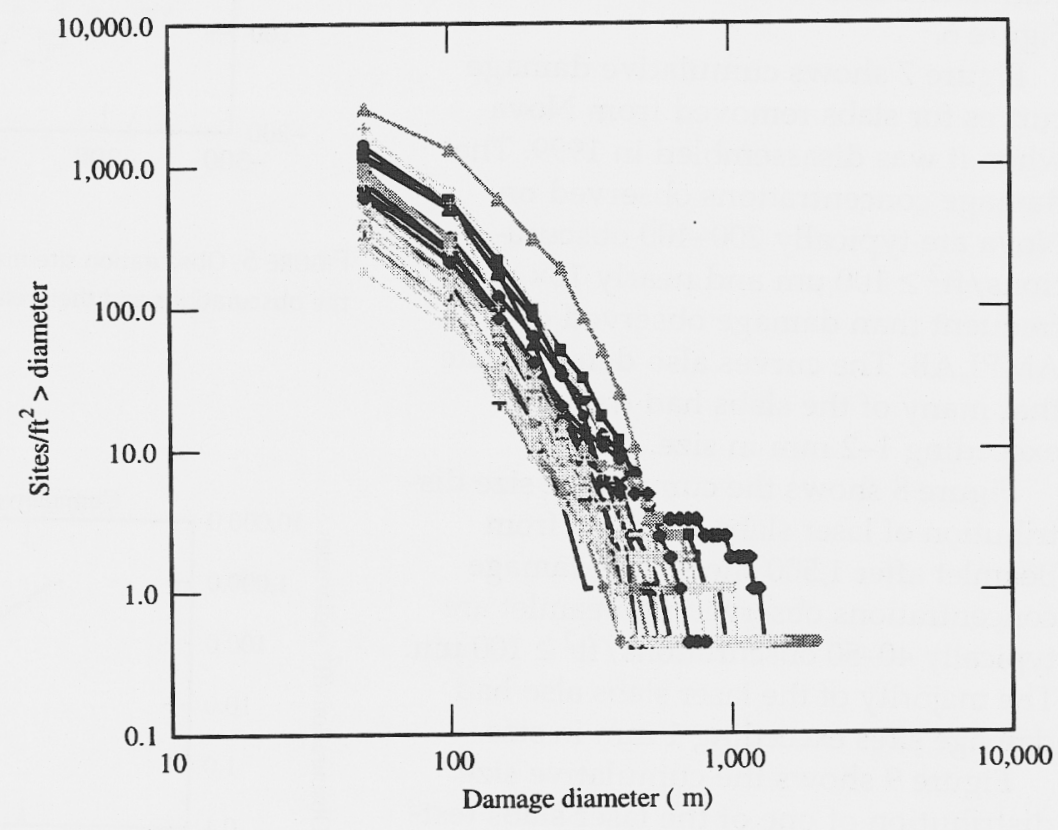

Figure 9. Cumulative size distribution of damage sites on slabs removed from AMPLAB. Concentration at $\geq 100 \mu \mathrm{m}$ is approximately 10 obscurations/ $\mathrm{ft}^{2}$. (40-00-1100-6154pb01)

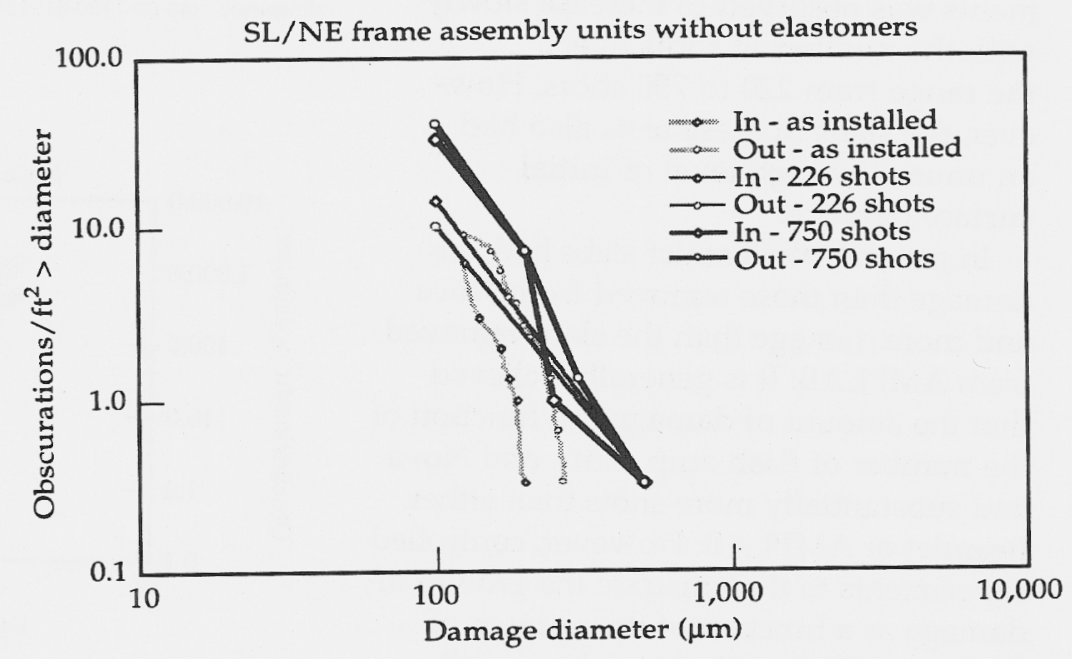




\section{Design and Operational Strategies for Contaminant Mitigation}

Several possible design and operational strategies are being implemented to reduce slab damage.

- Improved cleaning processes to remove residual organic films from structural surfaces.

- Elimination of visible elastomers within the amplifier cavities.

- Development of an amplifier flushing system to sweep aerosols from the slab cavity.

- Institution of an amplifier burn-in procedure to remove residual contaminant.

Improved cleaning of enclosure walls will be provided by industrial experts who will utilize a high-pressure spray water washing system incorporating a demonstrated surfactant. The detergents from the Brulin Corp. when applied hot and thoroughly rinsed with high-pressure deionized water at 2,500 psi or washed in a high-energy-density ultrasonic cleaner have shown to consistently produce surfaces with particle concentrations of $\leq$ Level 100 and organic levels of $\leq 0.1 \mu \mathrm{g} / \mathrm{cm}^{2}$, which is equivalent to only a few monolayers of organic material.

The elimination (or shielding) of elastomers from any region where they would see high-fluence flashlamp light is planned as well as the use of elastomers that are inherently low in aerosol production. Extensive small-scale experiments have shown that elastomers should be first selected by exposure to full-fluence $\left(10 \mathrm{~J} / \mathrm{cm}^{2}\right)$ flashlamp light and then evaluated for aerosol production at various reduced flashlamp fluence levels. The elastomers will then be shielded with metal where possible to produce an aerosol contributing only a few percent of the aerosol expected from the entire amplifier. Current estimates are that after the 200-shot burnin, an amplifier should be producing a peak aerosol of between Class 1,000 and Class 10,000 that will remain in the amplifier for less than 10 minutes once the flushing system is activated. Slab cavity flushing is accomplished by diverting $10 \%$ of the flashlamp cooling gas through the slab cavity immediately after a flashlamp firing.

Flashlamp generation of aerosols was first observed during the AMPLAB experiments and provides a mechanism for reducing organic films by allowing them to be pyrolyzed after the amplifier is completely assembled but before laser slabs are installed. Commissioning activities will include $\approx 200$ firings to monitor the progressive reduction of aerosols before the slab cassettes are installed.

\section{Notes and References}

1. Joseph Menapace: Slab damage induced by seeding laser glass with various contaminants and exposing them to limited flashlamp exposure.

2. MIL-STD-1246C, Product Cleanliness Levels and Contamination Control Program, Institute of Environmental Sciences and Technology, $940 \mathrm{E}$. Northwest Highway, Mt. Prospect, IL.

3. Irving Stowers et al., Achieving and Maintaining Cleanliness in NIF Amplifiers, Lawrence Livermore National Laboratory, Livermore, CA, UCRL-JC-130040. Prepared for the Third Annual International Conference on Solid State Lasers for Application to Inertial Confinement Fusion, Monterey, CA, June 7-12, 1998.

4. U.S. Federal Standard 209E, Airborne Particulate Cleanliness Classes in Cleanrooms and Clean Zones, Institute of Environmental Sciences and Technology, 940 E. Northwest Highway, Mt. Prospect, IL.

5. Clay Widmayer and Paul Renard, Survey of Damage Threat to Vacuum Barriers by Defects and Inclusions in NIF Optical Components, Lawrence Livermore National Laboratory, Livermore, CA, NIF-0088158, Mar. 15, 1998.

6. Clay Widmayer, Ken Manes, John Hunt, and Jack Campbell, Safety of NIF Vacuum Barriers from Failure Due to Laser-induced Damage, Lawrence Livermore National Laboratory, Livermore, CA, NIF-0034740, Sept. 7, 1999. 


\section{FY99 Preamplifier Module Engineering Prototype Activities}

In fiscal year 1999, a series of experiments demonstrating the performance of the preamplifier module (PAM) engineering prototype were completed. The engineering prototype is based on the same line-replaceable unit (LRU) package envelope as the current baseline production PAMs. These experiments also employed the master oscillator prototype. The purposes of these integrated experiments were to

- Demonstrate key NIF performance requirements using the capabilities of the equipment available.

- Identify problems in the baseline design and implement corresponding engineering changes.

The NIF requirements for the PAM and recent performance measurements of the engineering prototype are shown in
Table 1. A conceptual layout of the PAM is presented in Figure 10 and corresponding photographs are shown in Figure 11. Based on the prototype measurements, we have identified several engineering design changes, which we have implemented in our first article PAM design. These modifications will be validated by the first article performance measurements next fiscal year. Specifically, the engineering changes that have been implemented to improve performance include:

- Redesigning the vacuum relay telescope lenses and hand figuring the $5-\mathrm{cm} \mathrm{Nd:glass} \mathrm{rod} \mathrm{to} \mathrm{improve}$ beam quality.

- Adding a second Pockels cell slicer to reduce the prepulse leakage out of the regen from $100 \mathrm{~kW}$ to less than the required $30-\mathrm{kW}$ specification.

- Reengineering the optical mounts and $5-\mathrm{cm}$ rod amplifier nitrogen purge outlet to reduce beam pointing instabilities.

TABLE 1. PAM specifications and prototype performance at output.

\begin{tabular}{|c|c|c|}
\hline Performance parameter & NIF specification & Demonstrated \\
\hline Energy \& stability & $30 \mathrm{~mJ}-18 \mathrm{~J} @ 3 \% \mathrm{rms}$ & $18 \mathrm{~J} @ 2.6 \% \mathrm{rms}$ \\
\hline $\begin{array}{l}\text { PRF (@ } 18 \text { J) } \\
\text { Regen PRF }\end{array}$ & $\begin{array}{l}1 \text { per } 20 \text { minutes } \\
1 \mathrm{~Hz}\end{array}$ & $\begin{array}{l}1 \text { per } 10 \mathrm{~min} \\
1 \mathrm{~Hz}\end{array}$ \\
\hline Beam size & $30 \times 30 \mathrm{~mm}^{2}$ & $30 \times 30 \mathrm{~mm}^{2}$ \\
\hline Spatial beam shape & $2: 1$ & $2: 1$ \\
\hline Pulse length & $0.2-20 \mathrm{~ns}$ & $8 \mathrm{~ns}$ \\
\hline Pulse dynamic range & 125:1@18 J & Not measured \\
\hline SPD square pulse distortion & $\leq 2.3 @ 18 \mathrm{~J}$ & $2.3 @ 18 \mathrm{~J}$ \\
\hline $\begin{array}{l}\text { ASE @ 9 J @ PAM output } \\
\text { Prepulse @ } 9 \mathrm{~J} \\
\text { (1.5 J/beamline at injection) }\end{array}$ & $\begin{array}{l}\leq 30 \mathrm{~kW} \text { in } 3 \mathrm{~ns} \\
\leq 30 \mathrm{~kW}, \leq 120 \mu \mathrm{J}\end{array}$ & $\begin{array}{l}48 \mathrm{~kW} \text { in } 3 \mathrm{~ns} \\
100 \mathrm{~kW}, 140 \mu \mathrm{J}\end{array}$ \\
\hline Near-field contrast uniformity & $\leq 5.8 \% \mathrm{rms}$ & $7 \% \mathrm{rms}$ \\
\hline Wavefront $(\leq 3$ rd order) & $\leq 0.9$ wave & 1 wave \\
\hline $\begin{array}{l}\text { Divergence (encircled energy } \\
\text { radius) referenced to } 372-\mathrm{mm} \\
\text { beam. [After }<0.9 \text { wave of } \\
<3 \text { rd order correction] }\end{array}$ & $\begin{array}{l}\geq 80 \% \text { in } 4.65 \mu \mathrm{rad} \\
\leq 0.4 \% \text { outside } 33 \mu \mathrm{rad}\end{array}$ & $\begin{array}{l}>80 \% \text { in } 4.6 \mu \mathrm{rad} \\
\text { Not measured }\end{array}$ \\
\hline $\begin{array}{l}\text { Pointing stability } \\
\text { (372-mm beam) }\end{array}$ & $\leq 0.46 \mu \mathrm{rad} \mathrm{rms}$ & $2.6 \mu \mathrm{rad} \mathrm{rms}$ \\
\hline Beam rotation & $5 \mathrm{mrad} \mathrm{rms}$ & Not measured \\
\hline Power balance (PAM + MOR) & $<3 \% \mathrm{rms}$ & $3 \%$ rms@17 J square \\
\hline Shot-to-shot timing jitter & $8 \mathrm{ps} \mathrm{rms}$ & Not measured \\
\hline FM-AM conversion & $10 \% \mathrm{rms}$ & Not measured \\
\hline
\end{tabular}




\section{FY00 Front-End Integrated System Test}

An important activity we are planing for this fiscal year (FY00) is the front-end integrated system test (FEIST). FEIST is the first integration of several NIF systems. Specifically, FEIST will test in an integrated manner the first article master oscillator (MOR), the PAM engineering prototype, the input sensor package (ISP) prototype, and the integrated computer control system (ICCS). The specific goals of FEIST are to

- Test key NIF control hardware including the integrated timing system (ITS), delay generators, and networks.

- Validate ICCS hardware and software under true operational conditions.

- Demonstrate MOR operation including first article fiber oscillator, fiber amplifiers, stimulated Brillouin scattering (SBS) fail-safe, and pulse shaping.

- Demonstrate energy and alignment control of PAM with ISP.

- Demonstrate safety systems including SBS fail-safe, power conditioning, and laser shutters.

- Develop and validate acceptance test (ATP) and operational (OTP) procedures.

- Train operations personnel.

Several FEIST milestones have been met including

- Demonstration of FEIST 1 software release with surrogate control hardware.

- Integration of ICCS and ISP systems demonstrating alignment control of the ISP.

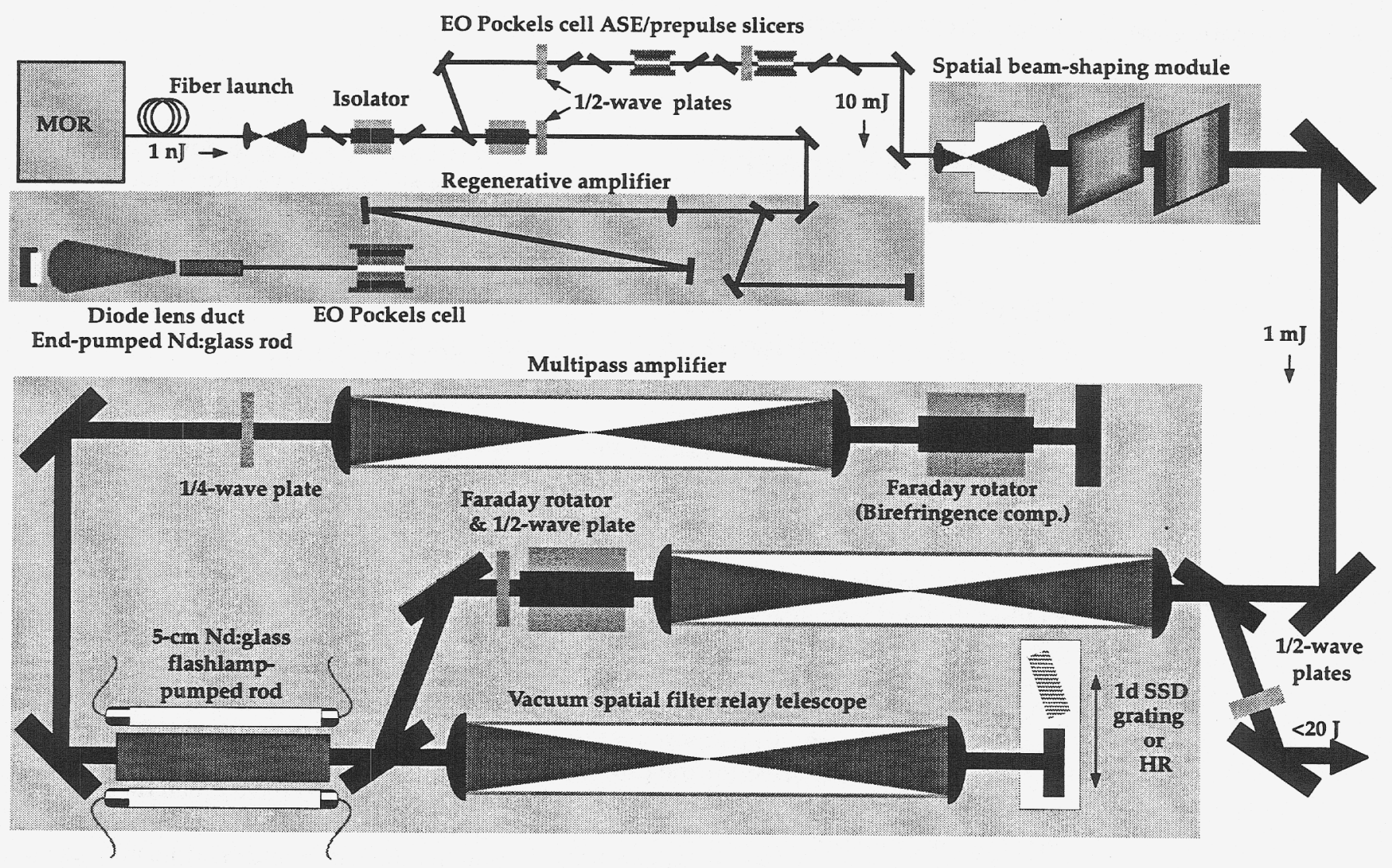

FIGURE 10. PAM system layout. (08-00-0500-2749pb01) 


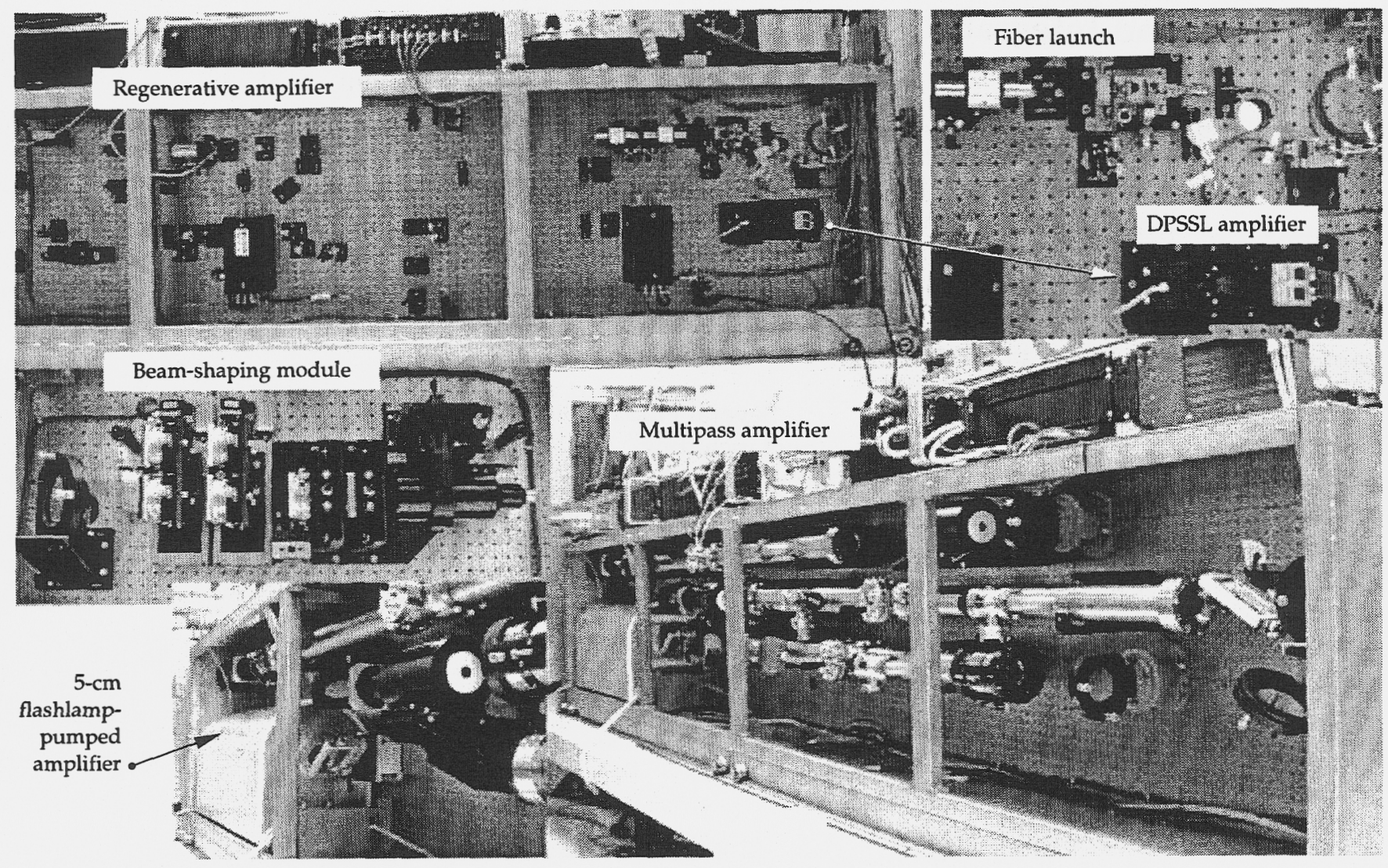

FIGURE 11. PAM engineering prototype. (08-00-0500-2750pb01)

\section{Low-Cost Capacitor Development}

\section{Objective of Low-Cost Capacitor Development}

The main amplifier power conditioning system (PCS) (see Figure 12) for the National Ignition Facility can store over $380 \mathrm{MJ}$ of energy for driving the nearly 8000 flashlamps. This energy is stored in 4600 individual high-energy discharge capacitors capable of storing over $83 \mathrm{~kJ}$ each. Four years ago, existing capacitor designs that would meet NIF's requirements were estimated to be $\$ 5 \mathrm{~K}-\$ 7 \mathrm{~K}$ each, or $\$ 23 \mathrm{M}-\$ 32 \mathrm{M}$ for the entire NIF PCS system. Capacitors were the single largest cost item for the power conditioning system, so investing development dollars in reducing the cost of energy storage capacitors had the potential for significant payoff. The goal of the devel- opment activities was to develop capacitor designs at multiple vendors that would meet the NIF requirements at $\$ 0.045 /$ joule or $\$ 3750 /$ capacitor in 1996 dollars or $\$ 4038$ / capacitor in 1999 dollars.

\section{Description of Development Plan}

Four vendors, two in the U.S. and two foreign, were identified that had both the interest and the capability to supply capacitors for NIF. Cost goals, design approaches, and funding methods were developed with each individual vendor. The first objective of the development effort was to demonstrate the required capacitor performance with a "low technical risk, higher cost" self-healing capacitor design. The second objective was to develop a very aggressive capacitor design that would have much higher technical risk. Developmental capacitors were built by the respective vendors and tested at LLNL. The number of iterations varied from 


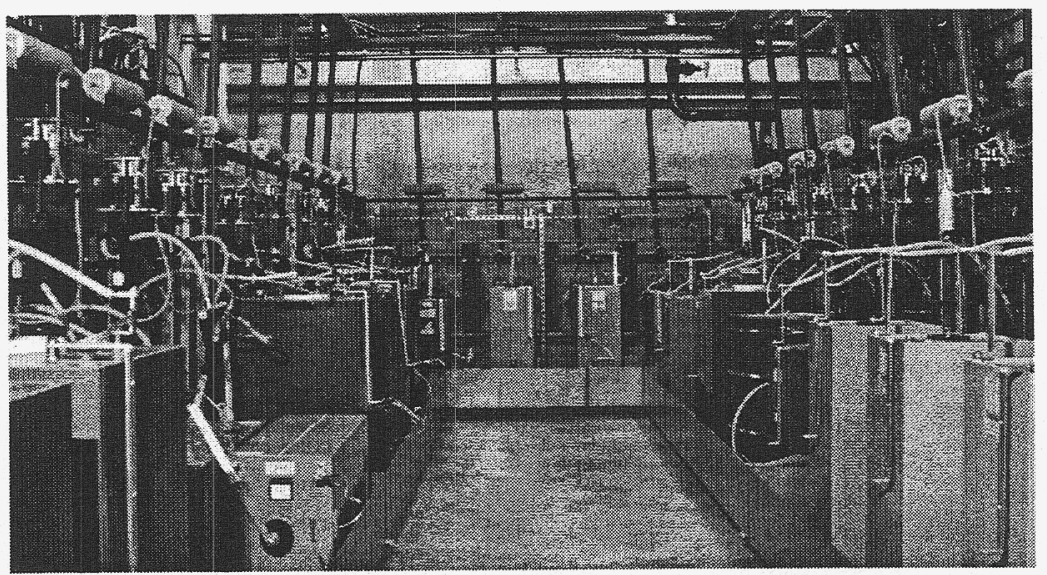

FIGURE 12. The NIF capacitor test and qualification system.

(08-00-0500-2741pb01)

vendor to vendor, and the success of different designs was mixed. The final goal of the development efforts was for every vendor to have two capacitor designs that could be qualified for NIF procurement, one low risk and one low cost.

\section{Summary of Results}

An extensive capacitor qualification testing program was conducted during FY1999 to qualify a set of capacitor designs for the NIF capacitor procurement. Three out of the original four vendors were able to qualify a capacitor design. After the qualification phase was completed, a request for quotes (RFQ) was released for procurement of the NIF capacitors. At the end of September 1999, an order was placed with ICAR, SPA in Italy for 1200 NIF capacitors with an option to award another 1200 capacitors at a fixed cost of $\$ 3750$ each. In early October, a second order was placed with Maxwell Technologies for 1200 capacitors at a fixed cost of $\$ 3950$ each with an option for an additional 1200 capacitors a a fixed cost of $\$ 4050$ each. The lifetime performance of both the ICAR and Maxwell capacitor designs exceeds the NIF requirements by at least a factor of 2 .

\section{High Bandwidth UV Fiber Development}

In June 1999, several years of fiber development culminated with the signing of a contract with the Vavilov State Optical
Institute, St. Petersburg, Russia, for the production of fiber for the NIF $3 \omega$ power diagnostic. This 28-month contract had two parts: the first four months were devoted to activating a computer-controlled fiber preform manufacturing station, the remaining 24 months were devoted to producing the NIF fiber. Approximately $2600 \mathrm{~m}$ of graded-index, 435 - $\mu \mathrm{m}$-core-diameter fiber, 308 cables in five individual lengths, were to be delivered.

Several prototype cables drawn from preforms manufactured using the new control system were delivered in 1999, as well as were similar cables from a pilot run. These cables ranged in length from 1 to $30 \mathrm{~m}$. Our bandwidth and attenuation measurements of these fibers (Figures 13 and 14) indicated that these fibers had excellent UV transmitting characteristics. Table 2 compares the measured values with our requirements.

\section{NIF Wavefront Control System}

The use of lasers as the driver for inertial confinement fusion and weapons physics experiments is based on their ability to produce high-energy short pulses in a beam with low divergence. Indeed, the focusability of high-quality laser beams far exceeds alternate technologies and is a major factor in the rationale for building high-power lasers for such applications. The NIF includes a wavefront control system to correct laser aberrations to yield a 


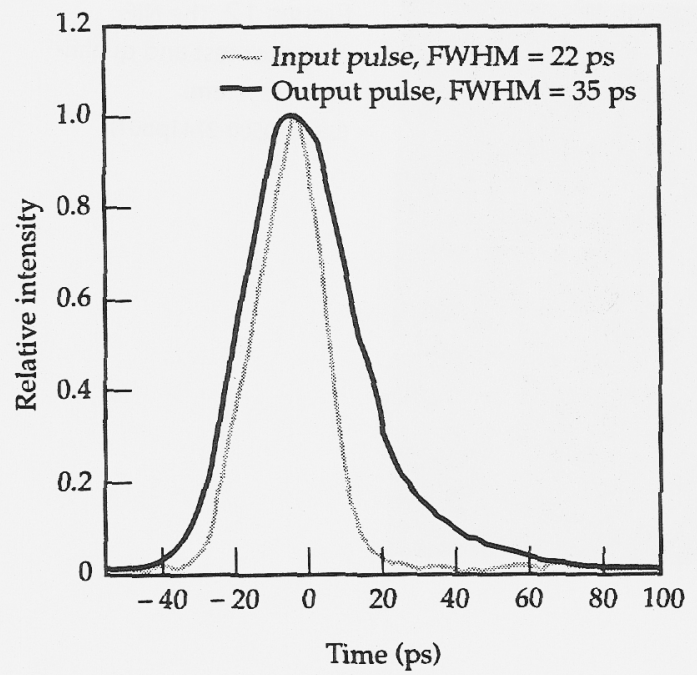

FIGURE 13. Test of a combination of 435 - $\mu \mathrm{m}$-core fibers in November 1999. The combination was $30+30+7+$ $1 \mathrm{~m}$ in length. The test was done by first measuring the width of 7 input pulses (average: 20.7 ps) and then measuring the width of 6 pulses (average: $33.5 \mathrm{ps)}$ ) that had propagated through the fiber. The dispersion calculated from the mean values was $0.4 \mathrm{ps} / \mathrm{m}$, one of the best performances observed. Other fiber combinations tested did not have such good performance but did meet the specification. (08-00-0500-2742pb01)

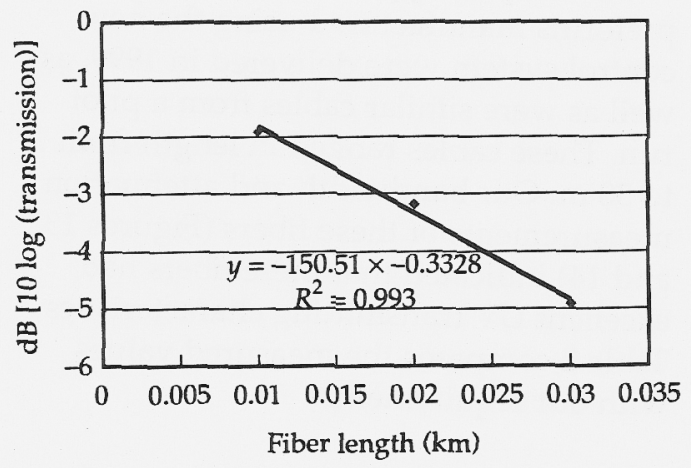

FIGURE 14. Three fibers each having a core diameter of $435-\mu \mathrm{m}$ and having nominal 10-, 20-, and 30-m lengths were measured independently in November 1999. The data and a least-squares fit are plotted; the slope of the fit is the attenuation: $\sim 150 \mathrm{~dB} / \mathrm{km}$. (08-00-0500-2743pb01)

TABLE 2. UV transmission characteristics of prototype cables compared with NIF requirements.

\begin{tabular}{lll}
\hline Characteristic & \multicolumn{1}{c}{ Requirement } & Performance \\
\hline Bandwidth & $<0.9 \mathrm{ps} / \mathrm{m}$ (goal: $\leq 0.5 \mathrm{ps} / \mathrm{m})$ & $0.5 \mathrm{ps} / \mathrm{m} \pm 0.15$ \\
Attenuation & $<200 \mathrm{~dB} / \mathrm{km}$ (goal: $<150 \mathrm{~dB} / \mathrm{km})$ & $\sim 150 \mathrm{~dB} / \mathrm{km}$ \\
\hline
\end{tabular}

focal spot small enough for these applications. Sources of aberrations to be corrected include prompt pump-induced distortions in the laser amplifiers, previous-shot thermal distortions, beam off-axis effects, and optics distortions due to gravity, mountings, and coatings. Aberrations from gas density variations and opticmanufacturing figure errors will also be partially corrected.

A block diagram of the NIF main laser optical system is shown in Figure 15, with the NIF wavefront control system components highlighted in black. Wavefront control functions are implemented as follows. A $1 \omega \mathrm{CW}$ probe beam is co-aligned with the NIF beam prior to injection into the main laser. The probe beam follows the NIF beam path. A 39-actuator large-aperture deformable mirror (DM) operates at the far end of the laser cavity. At the transport spatial filter (TSF) output, a tilted sampling surface reflects a small fraction of the beam towards a pick-off mirror near ISF focus that sends the sampled beam through relays to the output sensor. Within the output sensor, a 77-lenslet Hartmann sensor (HS) measures the wavefront. The Hartmann sensor's video output is read by a frame-grabber in the wavefront control computer. The computer calculates the surface displacements to be applied to the deformable mirror to correct the wavefront aberrations in the beam.

This year, the major elements of the wavefront control system were validated in the laboratory. The laboratory measurements were used to build models of system components for use in a computer model of the NIF laser propagation. The propagation model was used to validate whether NIF will be able to achieve its goal for spot size and on-target power.

The most expensive and difficult-to-produce component of the wavefront control system is the deformable mirror (DM). Characterization tests of a prototype NIF DM were completed this year. A photograph and a concept sketch of the prototype are shown in Figure 16. The DM must meet stringent performance requirements and must operate in a severe environment. A particularly stringent performance goal is that the DM should have less than 0.025 wave of $\mathrm{rms}$ residual error between the 


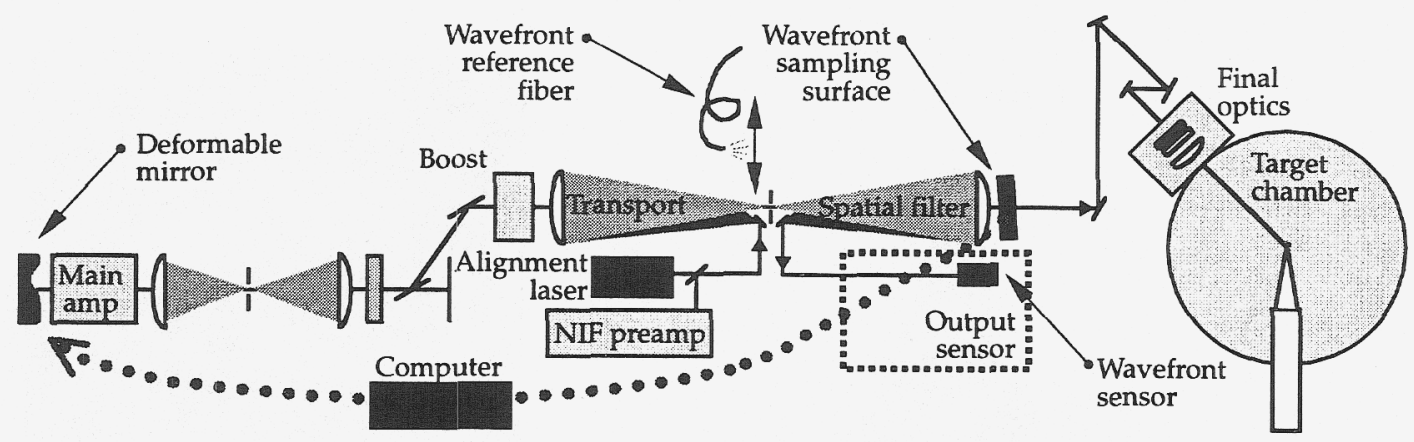

FIGURE 15. Overview of components in the NIF. (08-00-0500-2744pb01)

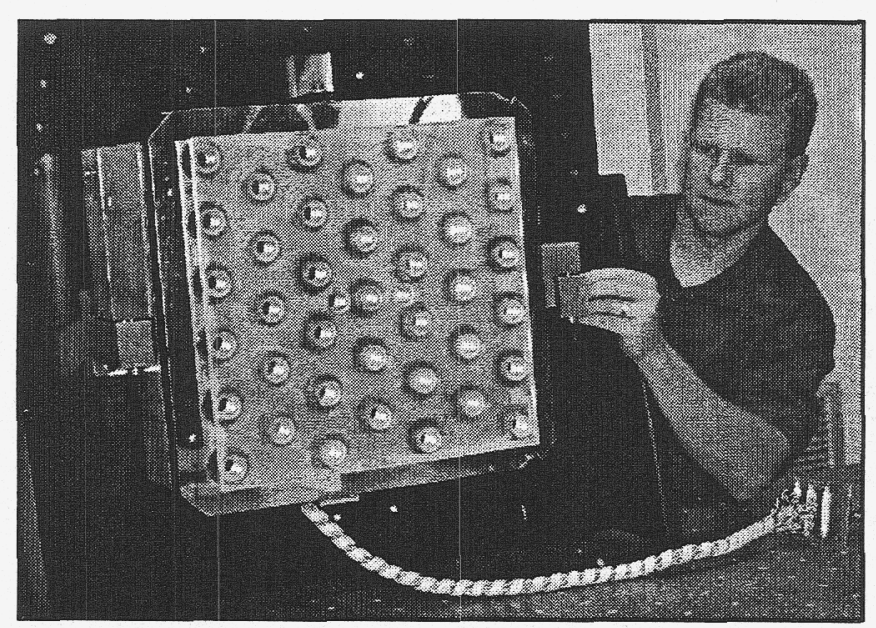

FIGURE 16. Photograph and concept sketch of the NIF deformable mirror showing major components. (08-00-0500-2745pb01)

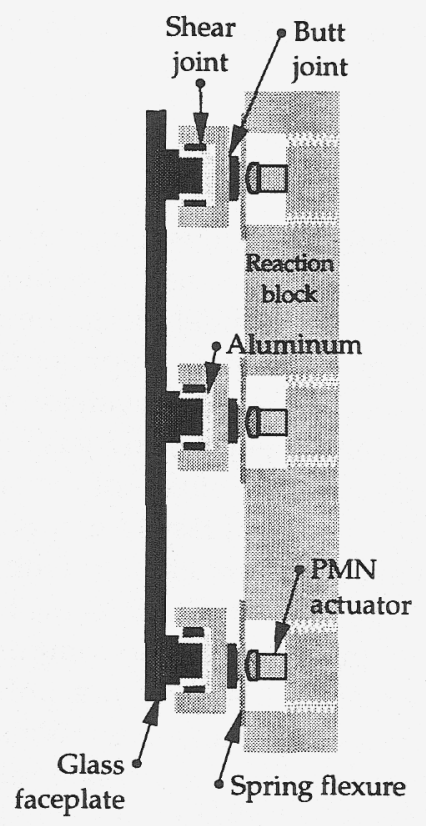

DM surface and a true flat surface when the mirror is commanded to be flat in closed loop operation. A particularly severe environmental parameter is the $10-\mathrm{J} / \mathrm{cm}^{2}$ flashlamp fluence that the DM must survive.

The DM tests were designed to generate data from which to build the DM model. The model was created from its measured influence functions and measured residual error. An influence function is the mirror surface shape when a single actuator is displaced, as shown in Figure 17a. The residual error is the actual mirror surface, when it has been commanded to be flat (in closed loop operation), as shown in Figure $17 \mathrm{~b}$. The DM surface is then modeled as the best-fit to the desired shape (as measured by the Hartmann sensor) of the superposition of the 39 measured actuator influence functions, plus the measured residual error.

The propagation model used to validate the NIF system performance was constructed from the Prop92 laser propagation code. The program was augmented to include all significant sources of aberration in the NIF, including elements based on analysis and on measurements of prototype and sample components. Model elements based on analysis included all optics, beam tilts and offsets, mounting aberrations (from finite element analysis models), and the real-time wavefront correction algorithm including Hartmann 
(a)

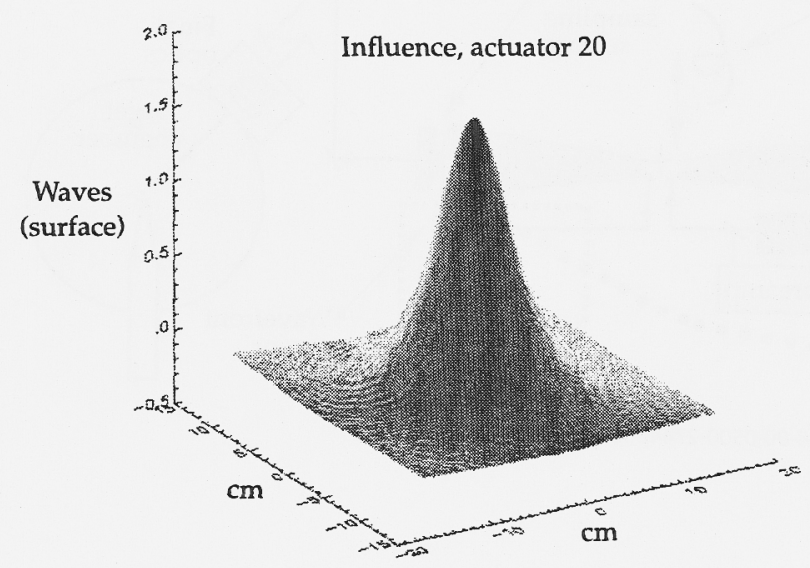

(b)

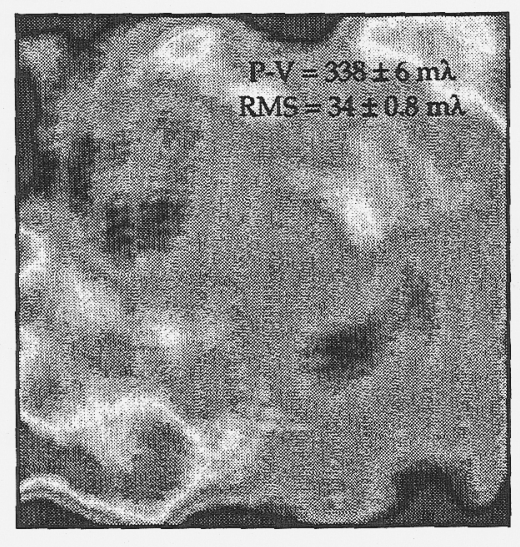

FIGURE 17. (a) Typical measured influence function and (b) typical measured residual error. (08-00-0500-2746pb01)

sensor limitations, detector thresholding, and baseline actuator positions. Elements based on Beamlet measurements included prompt and slow amplifier thermal distortions, frequency conversion with nonlinear wavefront effects, and gas inhomogeneity including the effects of the " $t_{0}-1$ " system (a NIF system of fast actuators that allows wavefront correction to within one second of the laser shot). Elements based on measurements of sample components included large-optic figure errors, polarizer and mirror coating stress aberrations, transport and final-optics assembly aberrations, and the DM. The full-fluence, far-field performance of the NIF based on the complete model is shown in Figure 18. This analysis shows that with the baseline components, the system will deliver $410 \mathrm{TW}$ into a $250-\mu \mathrm{m}$ target, compared to a goal of 500 TW. For comparison, without the wavefront control system (and with only low-spatial-frequency NIF aberrations included in the model), the calculated target power is reduced by a factor of 3.6. Furthermore, the calculations show that without wavefront control, the beam will clip the spatial filters, which would probably cause damage.

From Figure $17 \mathrm{~b}$, it is apparent that the prototype DM did not quite meet its 0.025-wave residual error goal. A parameter study was made to assess the effect of
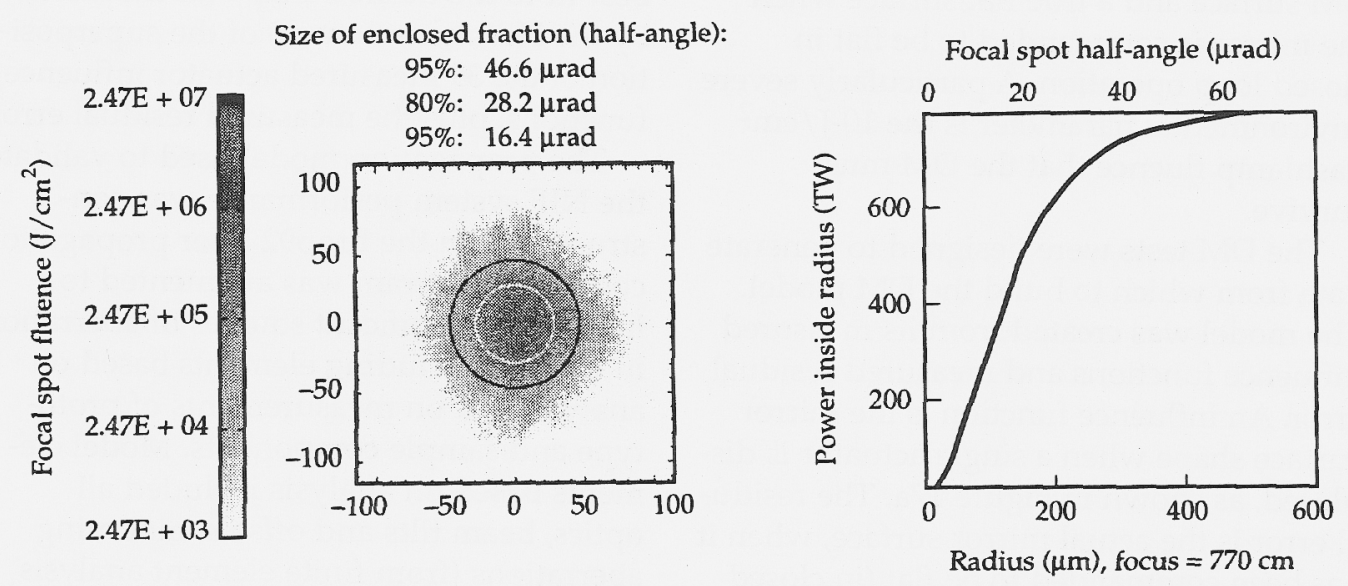

FIGURE 18. Predicted $3 \omega$ output far-field of the NIF using a complete system model and current baseline aberrations. (08-00-0500-2747pb01) 
the residual error on target power. The result of the study was that target power increases by only $1 \%$ when the residual error is decreased from the measured 0.034 wave to the goal of 0.025 wave. While efforts to reduce DM residual error will continue in pilot production, clearly other sources of aberration will need to be reduced for NIF to meet its on-target power goal.

To investigate the sources of aberration that do promote large increases in target spot size, a parametric study was conducted. This was done by cumulatively reducing each of the various sys- tem aberrations that contain significant high spatial frequencies. This included reducing the rms gradient specification for spatial frequencies with periods above $33 \mathrm{~mm}$ by a factor of $5 / 7$, reducing the of-order-10-mm-size optic figure errors by $13 / 17$, cutting front-end aberrations by half, and cutting turbulence by half or eliminating it entirely. The result is shown in Figure 19, which shows that turbulence and optic figure errors are major contributors to the NIF spot size. The NIF Project is investigating ways to mitigate turbulence and to minimize optic figure errors to keep the beams well focused onto the target.

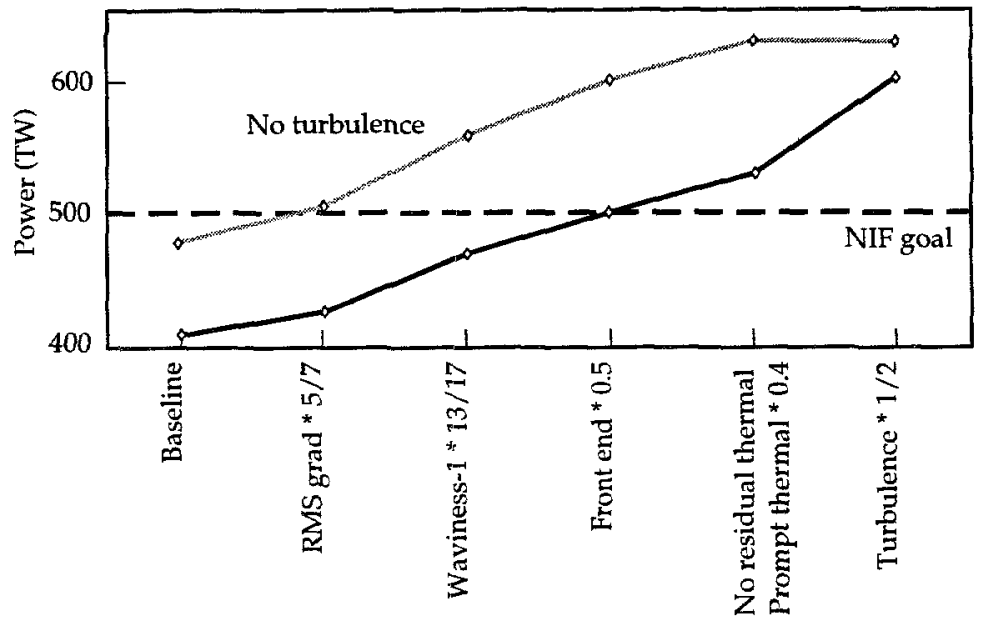

FIGURE 19. Sensitivity of predicted power to the NIF target as various aberration sources are cumulatively reduced. The predicted power is also shown with turbulence turned off in the model.

(08-00-0500-2748pb01) 



\subsection{Optics Technology Development}

$\mathrm{T}$ he National Ignition Facility (NIF) requires approximately 7500 largeaperture optics. These optics are divided into five main classes of materials:

- Nonlinear crystals (KDP, DKDP).

- Diffractive optics (phase plates and beam-steering optics).

- Gain media (laser glass).

- Transmissive optics (lenses and windows).

- Optical coatings (polarizers, mirrors, and antireflection coatings).

In 1995 we began a multiyear effort of development, facilitization, and pilot demonstration that culminates in the full production of these five classes of NIF optics beginning in about 2001. During 1999 we completed facilitization and in some cases began pilot operations. The pilot operations will continue into 2000 and 2001. This introduction summarizes some of the highlights from our 1999 effort.

To improve the yield of nonlinear crystals, we have investigated alternate crystal growth configurations. This requires a change in the seed geometry on which a crystal is grown. To date, the results are encouraging and we will continue to investigate this potential cost-saving technology for the NIF.

During 1999 we made the decision to no longer use a diffractive grating to achieve color separation. This is because the interference between the different diffractive orders of the color separation grating produces modulation in the beam intensity that is unacceptable. To solve this problem we have redesigned the $3 \omega$ NIF final optics assembly to include a wedged lens. This is an inexpensive and readily achievable fix to this difficult problem.

During Nova and Beamlet operation we discovered that organic contamination compromised the antireflection characteristics in silica sol-gel coatings. Recent work has shown that treatment of the sol-gel coatings with $\mathrm{NH}_{3}$ or $\mathrm{NH}_{3}$ plus hexamethyl disilazane (HMDS) reduces the adverse effects of organic contamination by reducing the microporosity in the coatings.

In 1999 we made significant progress finishing amplifiers, mirrors, and polarizers. Specifically we completed the facilitization at Zygo Corporation and have demonstrated that we can meet the stringent transmitted wavefront requirements for NIF using this novel high-speed finishing process. On average, the quality of the finished mirror substrates is about 30\% better than the NIF wavefront specification for peak-to-valley, rms gradient and power spectral density.

Pilot laser glass production began in 1999 and will continue into 2000. Early pilot operations showed that water uptake by both the glass and the hydroscopic glass raw materials can cause the laser glass to exceed the NIF OH specification. We have made appropriate changes to the pilot melting hardware and are now producing glass that is on average 20 to $50 \%$ lower than (i.e., better than) the NIF spec. We have also found that the 
FIGURE 1. Effect of boule asymmetry on $\mathrm{SHG}$ yield. Figure 1a shows a symmetrical boule, $55 \times 55 \times$ $55 \mathrm{~cm}$, with a maximum yield of seven SHG crystals. Figure $1 \mathrm{~b}$ shows an asymmetrical boule, $54 \times 57 \times 56 \mathrm{~cm}$, with two adjacent high sides and maximum SHG yield of 11 crystals. (40-00-0600-2886pb01)

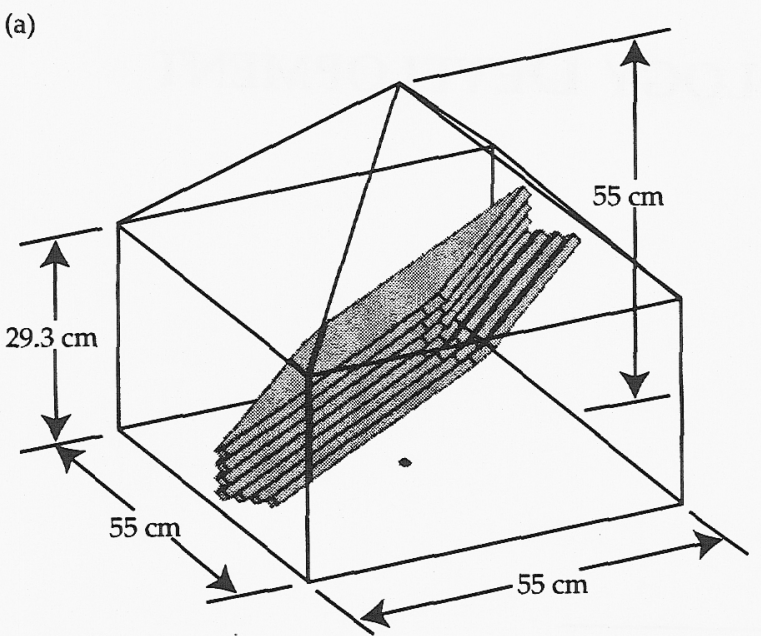

(b)

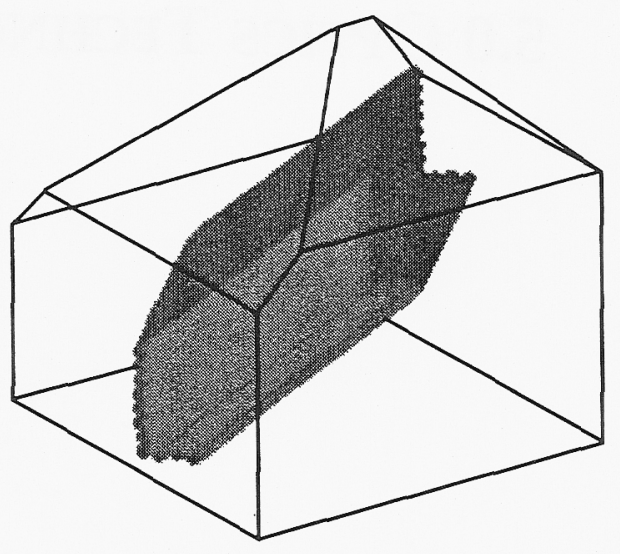

improvements in removing hydroxyl groups have led to improvements in the platinum inclusion count as well. We are optimistic that the yields from the pilot melting operations will exceed (when completed) our expectations.

Pilot operations are also proceeding at LLE and Spectra Physics on mirror and polarizer coatings and at Cleveland Crystals, Inc. (CCI) on rapid-growth KDP. Both pilot efforts are proceeding on schedule.

\section{Alternate Rapid-Growth Configurations of KDP Crystals for Improved Production Yields}

The final geometry of a KDP or KD*P boule has a large impact on maximum crystal yield. Current yield estimates used for production planning purposes are based on assumed symmetrical boules. The maximum yield of second-harmonicgeneration (SHG) crystals from a symmetrical boule is seven crystals. Small changes in geometry can increase this to between 11 to 14 crystals, resulting in cost savings due to fewer growth runs needed to produce the required $192+$ crystals for NIF. Figure 1 illustrates this difference in yield for a symmetrical (1a) vs an asymmetrical (1b) KDP boule of approximately the same dimensions and mass. Changes to seed geometry were studied as a means to repro- ducibly induce favorable asymmetry of the type seen in Figure 1(b), but the asymmetry was not reproducible and was uncontrolled. Nevertheless, we now have statistical data for the spontaneous occurrence of asymmetry and how it affects yield.

In the case of third-harmonic-generation (THG) crystals produced from $\mathrm{KD}^{*} \mathrm{P}$, a different type of asymmetry is desired. For vertical growth, the presence of a ridge at the top of the crystal is beneficial for two reasons: (1) it reduces the minimum boule height necessary to yield THG crystals, and (2) it increases the maximum possible yield of THG crystals. THG boules can also be grown in a horizontal configuration, as shown in Figure 2.

Changes to seed geometry were studied as a means to reproducibly induce the presence of a ridge at the top of the growing

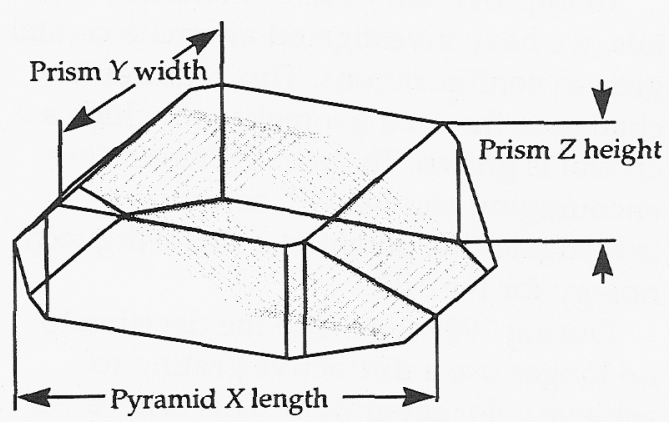

FIGURE 2. Horizontal boule geometry showing approximate layout of THG crystals. (40-00-0600-2887pb01) 
$\mathrm{KD} * \mathrm{P}$ boule. Results were inconclusive. Horizontal growth appears to be a more promising means to ensure consistent yields for rapid-growth $\mathrm{KD}^{*} \mathrm{P}$, and three NIF-size horizontal boules were grown. Similar to vertical growth, the limiting dimension appears to be the vertical (prism) height. A plot of the ratios of height/width and length/width for the three runs is given in Figure 3, along with black lines indicating the limits for nondestructive removal from the current platform. The first boule did require cutting the platform bars for removal because the boule became too wide before reaching the minimum height necessary for yield. The next two boules were close to but slightly above the minimum height/width ratio needed for benign removal.

\section{Assessment of Subaperture Color Separation Grating}

Beamlet experiments in fiscal year 1998 showed the deleterious cumulative effect of near-field modulation caused by diffractive optics, resulting in massive laser-induced damage to optics downstream. Modulation caused by the color separation grating (CSG), due to its deep $(1.4-\mu \mathrm{m})$ and dense $(110-\mu \mathrm{m}$ linewidth) feature size, was responsible for a sizable fraction of the damage observed. The modulation from interference between diffraction orders from the CSG was exacerbated significantly by the planarizing sol-gel antireflective layer applied over the surface topography by dip coating. It was clear that sol-gel coating of the CSG was not possible from a damage standpoint. It was proposed to confine the CSG to a circle approximately $15 \mathrm{~cm}$ in diameter centered on the diffractive optic plate, allowing natural dispersion of the symmetric focus lens to keep 1 and $2 \omega$ light out of the target location around the unpatterned area external to this circle. The plate could then be sol-gel coated except in this central circle containing the CSG, which would be bare. This compromise appeared to meet the objectives of color separation while maximizing the throughput of $3 \omega$ light. Another advantage of a subaperture CSG was that the linewidth could be coarsened by a factor of 3 to $\sim 350 \mu \mathrm{m}$, further reducing the magnitude and spatial scale of modulation.

At the start of fiscal year 1999, masks and substrates were procured for patterning test parts with the new, coarse-featured CSG patterns. A station for measuring the $1 \omega$ transmitted efficiency of these parts in diffraction orders of interest was set up. At this time, a retrenchment of the $3 \omega$ design

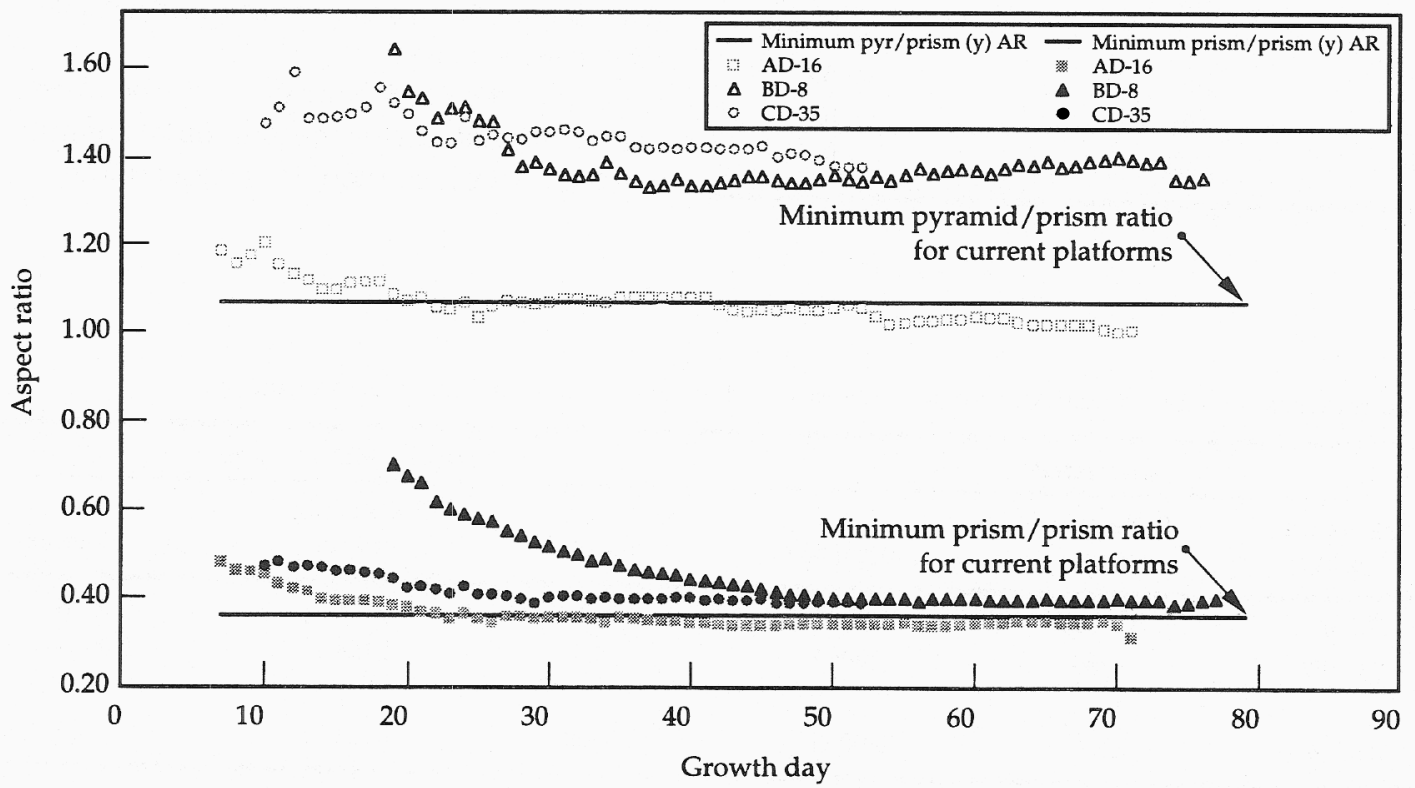

Figure 3. Plot showing aspect ratio as a function of growth day for LLNL horizontal KD*P growth runs. For the growth run where the aspect ratio dropped below the indicated minimum ratio, the platform was destroyed to remove the boule. (40-00-0600-2888pb01) 
of the final optics assembly began, and work on the CSG was halted. The result of this redesign effort has been summarized in memorandum NIF-0039912 issued Dec. 9, 1999. The proposed redesign has reverted back to an original one wherein a wedged lens is used to deflect the 1 and $2 \omega$ light from the target. A wedged lens eliminates the necessity of a CSG and also offers an inexpensive fix to a design oversight that resulted in a beam rotation mismatch between the beam transport assembly and the target chamber. An official design change request has been approved for this redesign, and work on a CSG is no longer being funded.

\section{Full-Size Stabilized Sol-Gel Antireflection Coatings on Fused Silica}

Organic contamination of porous antireflection coatings on large laser optics can be a significant contributor to loss of light transmission during service. Much of the affinity for organic contaminants originates in the large amount of microporosity

TABLE 1. Effect of vapor $\left(\mathrm{N}_{2} \mathrm{BET}\right)$ treatment on specific area and pore size in bulk and film sol samples.

\begin{tabular}{lcc}
\hline Sample & Specific area $\left(\mathrm{m}^{2} / \mathrm{g}\right)$ & Pore peaks $(\AA)$ \\
\hline As-deposited film & 174 & 70 \\
$\mathrm{NH}_{3}$-treated film & 76 & $135 \& 18$ \\
Evaporated bulk sol & 564 & 55 \\
$\mathrm{NH}_{3}$-treated bulk & 238 & 100 \\
$\mathrm{NH}_{3}+$ HMDS-treated bulk & 215 & 100 \\
\hline
\end{tabular}

within the 10- to 20-nm-diameter constituent silica particles of the sol coatings. Treatment of the sol coatings with aqueous ammonia vapor decreases the specific surface area (Table 1), largely by the removal of microporosity.

Additional treatment of the coating with HMDS (hexamethyl disilazane) vapor can further reduce the surface affinity for polar organics by coating a significant portion of the silica surface with nonpolar methyl groups. A significant benefit of the ammonia treatment alone is its improvement of the abrasion resistance of the coatings. ${ }^{1}$

To demonstrate the compatibility of a postdeposition vapor treatment with large laser optics, a Nova input spatial filter lens (SF7) was processed through ammonia and HMDS vapor treatments after deposition of the sol AR coating by the normal dip method.

No significant processing problems were discovered during the preparation of the lens, and so a simple scale-up of previous bench-scale experiments seems easily achievable for NIF-scale optics. A second, untreated lens was also placed into service in the same time frame to provide a basis for comparison.

The lenses were installed in Nova beamlines and remained online for approximately 290 shots at an average fluence of $4.3 \mathrm{~J} / \mathrm{cm}^{2}$ (maximum fluence of $8.4 \mathrm{~J} / \mathrm{cm}^{2}$ ) until the shutdown of Nova in June 1999. Table 2 shows the transmission and reflectance characteristics of the two lenses at the beginning and end of the laser service.

Under the best circumstances, the uncertainty in photometer measurements of this type is \pm 0.001 , so there is no discernible

TABLE 2. Reflectance and transmission characteristics of Nova SF7 lenses used in sol coating stabilization demonstration.

\begin{tabular}{|c|c|c|c|c|c|c|}
\hline & \multicolumn{3}{|c|}{ Untreated } & \multicolumn{3}{|c|}{$\mathrm{NH}_{3}+\mathrm{HMDS}$ treated } \\
\hline & $\begin{array}{l}\text { Reflectance- } \\
\text { input }\end{array}$ & $\begin{array}{l}\text { Reflectance- } \\
\text { output }\end{array}$ & Transmission & $\begin{array}{l}\text { Reflectance- } \\
\text { input }\end{array}$ & $\begin{array}{l}\text { Reflectance- } \\
\text { output }\end{array}$ & Transmission \\
\hline Beginning of service & 0.0001 & 0.0001 & 0.9982 & 0.0006 & 0.0007 & 0.9978 \\
\hline End of service & 0.0007 & 0.0001 & 0.9982 & 0.0006 & 0.0008 & 0.9983 \\
\hline Change & 0.0006 & 0.0000 & 0.0000 & 0.0000 & 0.0001 & 0.0005 \\
\hline
\end{tabular}


difference in basic optical performance of the coatings either between lenses or before and after service on Nova. The demonstration does indicate that there are no obvious problems with the modified coatings when used in the actual laser environment.

Obviously, there was insufficient residual contamination present in the Nova beam enclosures to determine the efficacy of the vapor treatment with respect to organic contamination resistance during the period of laser exposure for the SF7 lens experiment. A more detailed study of the effects of the coating treatments on the adsorption characteristics of the films was performed, however, and did clearly demonstrate the effectiveness of the treatments in reducing coating affinity for organic contaminants.

Figure 4 shows the relationship between the vapor phase concentration (expressed as relative pressure) and the amount of adsorbed dibutyl phthalate (DPB), an organic compound representative of pump oils and relatively volatile plasticizers, on sol-coated polished quartz. The measurements were made by direct gravimetric determination using a sol-coated surfaceacoustic-wave device ${ }^{2}$ with organic partial pressure controlled by the temperature of a pure liquid source.

The presence of high microporosity in the untreated coating is evident as a steep rise in adsorption near the origin accompanied by

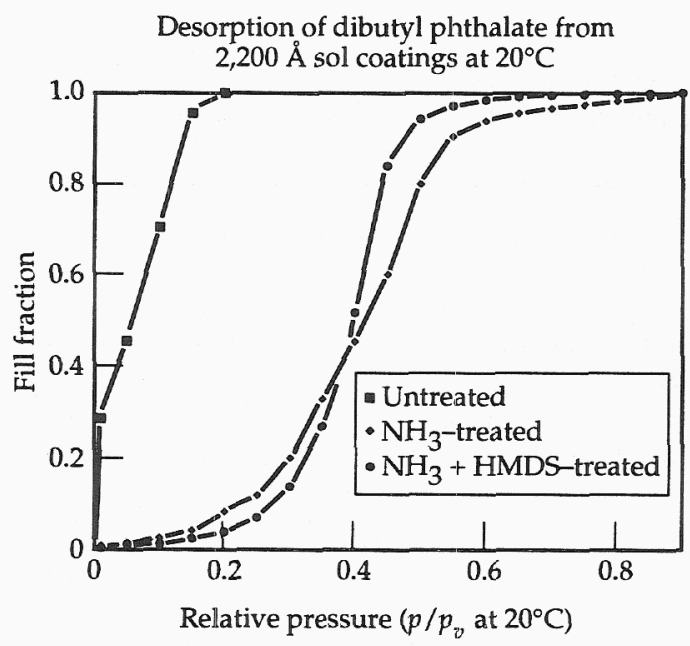

FigURE 4. Desorption isotherms at $20^{\circ} \mathrm{C}$ for dibutyl phthalate (DBP) on porous silica coatings. (40-00-0600-2890pb01)

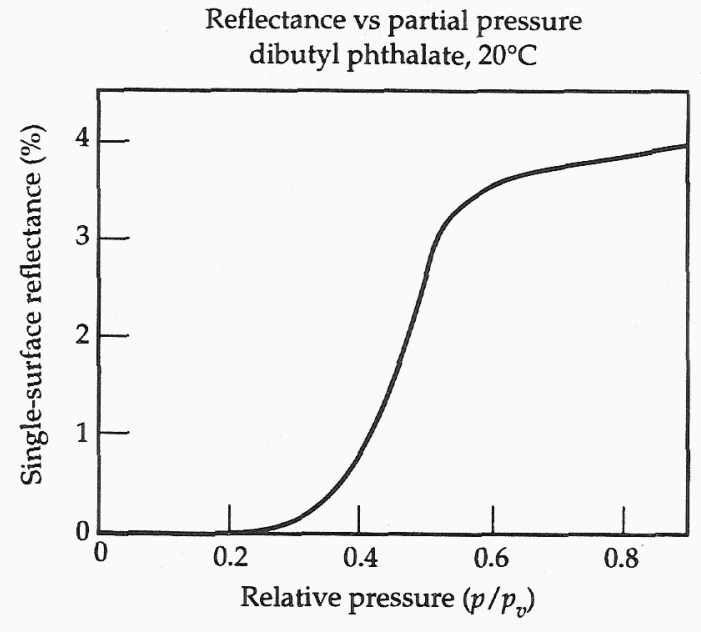

FIGURE 5. Reflectance loss versus relative pressure of dibutyl phthalate for ammonia-treated, $1 \omega$ sol antireflection coating. (40-00-0600-2891pb01)

low-pressure hysteresis, which accounts for an approximately $20 \%$ irreversible adsorption level when the untreated films are exposed to only trace amounts $\left(p / p_{v}<0.01\right)$ of DBP. In contrast, treated coatings show relatively little adsorption at low organic partial pressures and therefore admit some realistic operating margin in the presence of organics without unacceptable reflection loss (Figure 5).

Capillary condensation in the larger mesoporous regions between silica particles always dominates at higher partial pressures, limiting the allowable partial pressure of organics to approximately 0.2 of its pure component vapor pressure for treated films. This $0.2 p_{v}$ limit has therefore been adopted as the maximum allowable organic level inside sol-coated optic enclosures for the $\mathrm{NIF}^{3}$ under the assumption that postdeposition treatment of the antireflection coatings is performed.

\section{Notes and References}

1. P. F. Belleville and H. G. Flock, Sol-Gel Optics III, in Proc. SPIE (J. D. MacKenzie, ed.), vol. 2288, pp. 25-32 (1994).

2. H. Wohltjen, Sensors and Actuators, 5, 307-335 (1984).

3. J. Fair and C. Thorsness, Maximum Acceptable Organic Concentrations in NIF Enclosures Containing Sol-Coated Optics, Lawrence Livermore National Laboratory, Livermore, CA, NIF-0039225. 


\section{The NIF Cleaning Process for KDP-KD*P Crystals}

The NIF cleaning process for KDP-KD*P crystals must consistently remove the remnants of oils used during final diamondtool finishing of the optics, the KDP debris that results from the diamond flycutting process, and any particulate contamination that results from handling the optics prior to the application of the antireflection coating. Two identical cleaning systems have been built by Forward Technology Industries, Inc., for processing KDP crystals. One system (shown in Figure 6) has

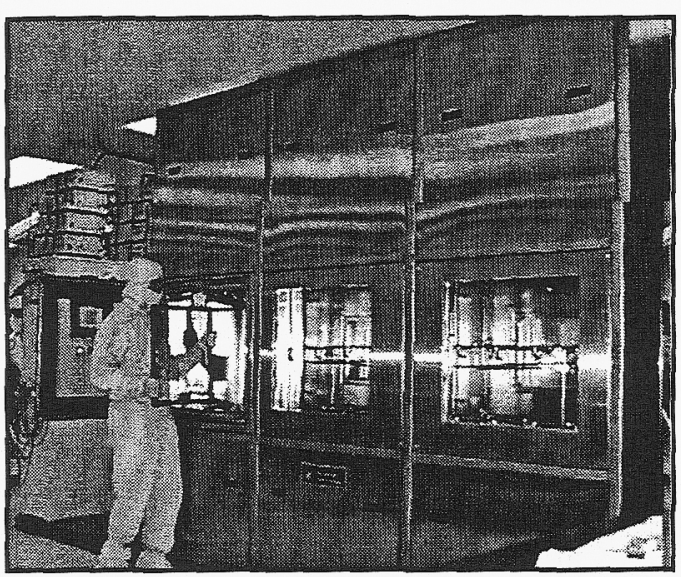

FIGURE 6. Optic being loaded into the Forward Technology Industries automated solvent cleaning system. (40-00-0600-2889pb01) been installed, commissioned, and is now operating at LLNL for final precision cleaning of all NIF crystals. The second system will be installed at the crystal finishing vendor. A Fourier transforminfrared reflectance (FT-IR) spectrometer capable of measuring organic contamination on full-size NIF KDP optics was also received. This instrument will be used to verify that NIF optics meet the $0.1 \mathrm{mg} / \mathrm{m}^{2}$ organic contamination specification after cleaning.

Cleaning process development focused in two areas this past year: (1) identification of appropriate solvents to remove the organic diamond-finishing oils, and (2) identification of process parameters and solvents to remove KDP debris and ambient particulate contamination. A sequence of more polar to less polar solvents (between 2-butanol, methyl isobutyl ketone [MIBK], and toluene) was tested for both organic contamination and particulate removal from freshly diamond-turned KDP surfaces. As seen in Table 3, toluene was the most effective solvent for removing a variety of expected or proposed diamond-turning-process organic contaminants. Several proposed additives, such as the Lubrizol 936 and Paratac 178, could not be successfully removed from the crystals by any choice of solvent. Hence, these additives will not be used for NIF crystals. As seen in Table 4, the Nova/Beamlet process of soaking plus spray was ineffective

TABLE 3. Toluene proved to be as effective or better than 2-butanol, methyl isobutylketone (MTBK), or a blend of 50:50 toluene and 2-butanol, at removing organic contamination from KDP crystals. Shaded boxes indicate residual contamination levels that exceed the NIF specifications.

\begin{tabular}{lccccc}
\hline & & \multicolumn{4}{c}{ Residual contamination $\left(\mu \mathrm{g} / \mathrm{cm}^{2}\right)$} \\
\cline { 5 - 6 } Contaminant & $\begin{array}{c}\text { Initial contamination } \\
\left(\mu \mathrm{g} / \mathrm{cm}^{2}\right)\end{array}$ & Toluene & $50: 50 \mathrm{mix}$ & 2-Butanol & MIBK \\
\hline None & 0 & 0.06 & $<0.01$ & 0.04 & 0.04 \\
Drakeol 7 & 14.0 & 0.02 & 0.02 & $<0.01$ & 0.02 \\
Lard Kut & 1.8 & 0.04 & 0.03 & 0.26 & 0.05 \\
Lubrizol 936 & 9.8 & 0.38 & 0.34 & 2.90 & 0.99 \\
Pneumo cutting oil & 11.4 & 0.08 & $<0.01$ & 0.45 & 0.07 \\
Current cutting oil & 11.5 & 0.03 & $<0.01$ & 0.51 & 0.03 \\
Human skin oil & 1.9 & 0.10 & 0.05 & 0.02 & 0.03 \\
Methyl silicone & 0.49 & 0.01 & 0.01 & 0.03 & 0.04 \\
Paratac 178 & 11.8 & 0.11 & 0.26 & 0.72 & 0.37 \\
\hline
\end{tabular}


TABLE 4. Ultrasonication and a final clean solvent spray were required to remove all of the particles left after diamond-finishing KDP optics.

\begin{tabular}{lccc}
\hline & \multicolumn{3}{c}{ Particle populations } \\
\cline { 2 - 4 } Cleaning process & $<5 \mu \mathrm{m}$ & $5-50 \mu \mathrm{m}$ & $>50 \mu \mathrm{m}$ \\
\hline As received & 34,300 & 2,740 & 229 \\
After toluene soak and spray & 34,300 & 2,740 & 251 \\
After ultrasonication & 20 & 2 & 0 \\
After ultrasonication and spray & 0 & 0 & 0 \\
\hline
\end{tabular}

at particulate removal. When the Nova process was combined with a short immersion in ultrasonic agitation, all of the solvents tested removed all particulate from these $4-\mathrm{cm}^{2}$ parts. Therefore, toluene has been selected as the single solvent that will be used for precision cleaning of NIF KDP/DKDP optics.

\section{Advanced Technology: Continuous Laser Glass Melting and Forming}

Advanced laser glass melting processes have been developed separately by Schott Glass Technologies Inc. and Hoya Corporation under work funded jointly by LLNL and the Centre d' Etudes de LimeilValenton. The two glass companies have chosen different development approaches. Schott has chosen to design and develop a full-scale melting system that will then become the production melter. The Schott approach allowed for one development run to verify equipment design and the melting and forming process. Such verification was completed in November and December 1997. In contrast, Hoya chose to carry out development using a subscale, continuous melter. Because of the smaller size and lower operating costs of their equipment, Hoya was able to carry out several melting and forming campaigns, which were completed in March 1998. Both vendors have completed their development efforts, and as of February 1999, they are in the midst of the first full-scale run that we term the "pilot." This work will be followed by several years of production, approximately 2 to 3 years for the NIF and about 3 to 4 years for the French Laser Megajoule (LMJ).
Many details of the manufacturing process are highly proprietary to each company. Therefore, we give only a generic description of the melting, forming, and coarse annealing process. Nevertheless, this description should provide an idea of the progress in laser glass manufacturing technology that has occurred as a result of the NIF and LMJ projects. The laser glass melting systems developed by Schott and Hoya are arguably the most advanced optical glass melting systems in the world.

As shown in Figure 7, a continuous optical glass melting system is generally divided into several interconnected zones. Each zone consists of one or more vessels designed to carry out a specific aspect of the process. For laser glass continuous melters, Figure 7 shows the six main processing zones, ${ }^{1}$ which are raw material batching, melting, conditioning, refining, homogenizing, and continuous strip forming. The six regions are interconnected, allowing for a continuous flow of glass from one zone to the next.

It is desirable that the raw materials be batched together and then thoroughly mixed in a dry atmosphere. The batch is then delivered continuously to the melter with precautions to avoid water uptake by hydroscopic raw materials. Batch powder that enters the melter dissolves in the molten glass and undergoes large-scale mixing. Off-gas handling equipment collects any gas emissions from the melter (or other vessels) and treats the effluent to meet environmental regulations.

Glass continuously flows from the melter into the conditioning unit, where the redox state of the melt is adjusted to enhance dissolution of $\mathrm{Pt}$ inclusions. If required, steps may also be taken to 
FigURE 7. Schematic of the continuous laserglass melting systems to be used to manufacture NIF and LMJ laser glass. The six process zones are discussed in the text. (4000-1100-6155pb01)

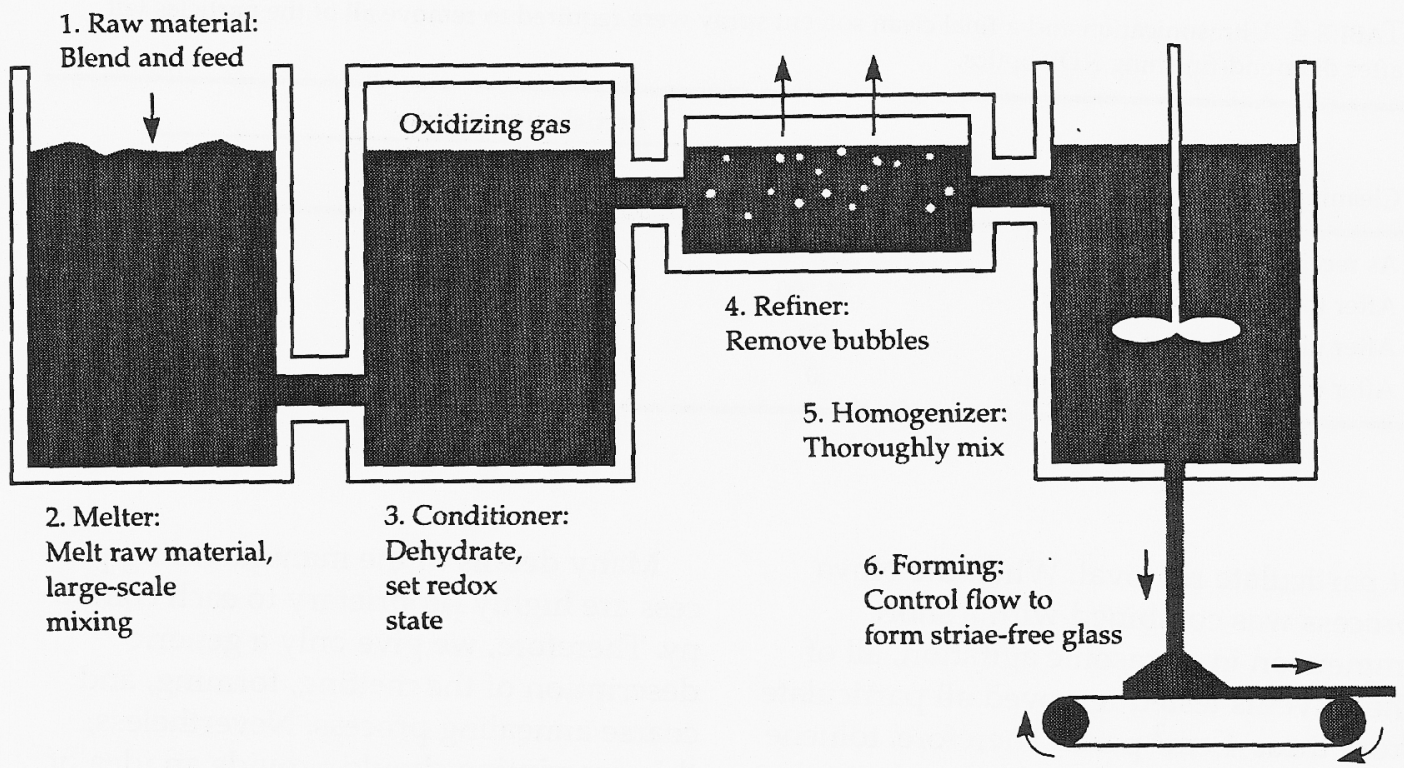

remove any excess "water" (i.e., hydroxyl groups) in the glass. Glass from the conditioning unit then flows to the refiner section where the temperature is generally elevated to reduce the glass viscosity and thereby increase the bubble rise velocity to promote bubble removal.

Glass from the refiner enters the homogenizing section, where Pt stirrers thoroughly mix the glass to achieve the part-per-million index homogeneity required for ICF laser applications. Just as in the discontinuous process, the temperature of the homogenizing section is reduced to adjust the glass viscosity to give the desired flow characteristics needed to form a wide, thick, homogeneous strip of glass. The width and thickness of the glass strip produced during the forming operation are greater than those of any optical glass ever produced prior to NIFand LMJ-driven glass development.

The glass manufacturers employ highly proprietary technology to "form" (i.e., cast) the glass into a homogeneous, continuous strip free of sharp index variations (striae). Once successfully formed, the cast strip moves by conveyor belt through a long (25- to 35-m), coarse annealing oven (Figure 8) where the temperature is ramped down at a rate to avoid generating unacceptable thermal stresses in the glass. Finally, the cast strip is cut into pieces that are individually processed to give the desired laser slab blank.

Both manufacturers will use advanced processing conditions designed to minimize the formation of $\mathrm{Pt}$ inclusions in laser glass. Prior to 1986, Pt inclusion damage represented the major source of damage in laser glass used for high-peak-power applications. However, new processing methods effectively reduce the $\mathrm{Pt}$ inclusion concentration by more than 1000-fold, to fewer than an average of 1 to 2 per laser glass slab (i.e., less than 0.1 per liter).

\section{Postprocessing}

Once the laser glass has been melted and formed into plates, several other process steps must be completed before the glass can be shipped to the final finishing vendor. Specifically, the laser glass needs to undergo prefabrication to a size suitable for inspection for striae and $\mathrm{Pt}$ inclusions. Next, the glass is slowly annealed to remove any residual strain, a process that can take many days. Finally, the glass blank is fabricated to the final dimensions, inspected for homogeneity, and prepared for shipping. 


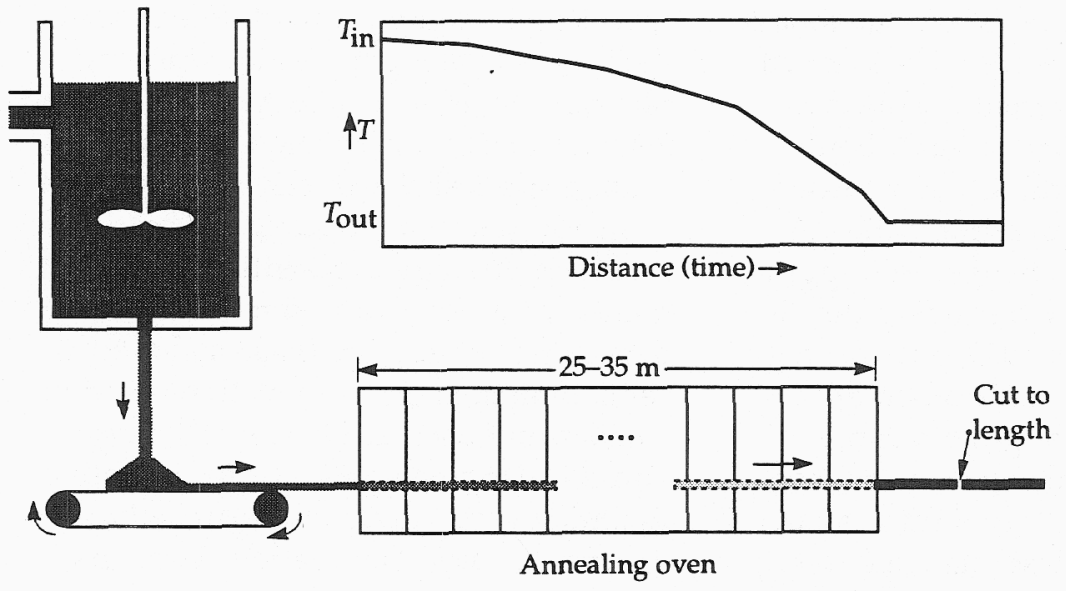

FIGURE 8. Schematic of the system used to coarse-anneal the as-cast, continuous laser glass strip.

(40-00-1100-6156pb01)

\section{Manufacturing Schedule}

Laser glass manufacturing for the NIF and LMJ is divided into two main phases: pilot and production. Pilot refers to the first production runs. Results from the pilot run will be used to establish yield and costs. In addition, glass from the pilot runs will be used by the finishing vendors to demonstrate the advanced laser glass finishing and polishing methods to be used in final production.

The production phase immediately follows the pilot phase. The first stage of laser glass production will be primarily for the NIF facility because NIF construction will occur earlier than that for the LMJ. The NIF production will take place over approximately three years at a rate of about 1000 slabs per year. NIF production will be followed by LMJ production, which will last three to four years. During the third year of production, the NIF and LMJ may overlap somewhat, requiring a short-term increase in the annual production rate.

\section{Summary}

The NIF and LMJ laser systems require about 3380 and $4752 \mathrm{Nd}$-doped laser glass slabs, respectively. Continuous laser glass melting and forming will be used for the first time to manufacture these slabs. Two vendors have been chosen to produce the glass: Hoya Corporation and Schott Glass
Technologies Inc. The laser glass melting systems that each of these two vendors has designed, built, and tested are arguably the most advanced in the world. The cost goal is to manufacture the laser glass for about $\$ 1000 / \mathrm{L}(\$ 350 / \mathrm{kg})$, or roughly a factor of 3 lower than the cost associated with current, one-at-a-time production methods. Production of the laser glass began on a pilot scale in the fall of 1998.

\section{Notes and References}

1. J. H. Campbell et al., J. Non-Cryst. Solids 263, 342-357 (2000).

\section{Rapid-Growth KDP Crystal Pilot}

Contracts for pilot production of KDP using the rapid-growth technique were awarded to two vendors, Inrad and Cleveland Crystals Inc. (CCI), in December 1998. The initial period of performance for each contract was seven months, but both contracts were extended at the request of the vendors, to permit completion of growth runs that were ongoing at the scheduled completion date of the contracts. Sixty-three percent of growth runs completed during the pilot period produced NIF-sized KDP boules. Direct training and observation by LLNL personnel at each vendor site were primary reasons for the successful implementation of rapid growth at commercial vendor 
sites. Initial growth runs failed due to lack of adherence to established LLNL growth procedures, which resulted in spurious crystallization. Some of the early boules produced contained minor flaws caused by high levels of solution impurities. A model describing the changes in impurity levels during the growth run was developed at LLNL in early 1999, but was not fully implemented or understood at the time of the first vendor growth runs. Subsequent growth runs have produced boules of high quality based upon visual inspection, although laser damage testing of rapid-growth boules from the vendors has not been performed. Both Inrad and CCI are now capable of producing NIFsized boules of KDP.

\section{Coatings Pilot at LLE and Spectra-Physics}

Awarding pilot production contracts was contingent on successful completion of facilitization at the coating vendors. By April 1998, the University of Rochester Laboratory for Laser Energetics (LLE) and Spectra-Physics were both awarded facilitization contracts to modify their facilities for NIF mirror and polarizer coating production. The facilitization activities included construction of metrology labs for laser conditioning, photometry, and interferometry instruments illustrated in Figure 9.

In concert with completion of the labs, the metrology tools were also being constructed and fielded at the coating vendors. The two laser-conditioning stations at each coating vendor were based on the PLATO conditioner used for Beamlet optics. They were, however, upgraded from a research tool architecture for understanding laser conditioning with experiments lasting several weeks to a production tool for processing approximately 1250 mirrors and polarizers at a rate up to one optic per day. Existing 18-in.-diameter interferometers used for OMEGA and Nova were upgraded with high-resolution cameras, and the 633-nm lasers were replaced with 1064-nm lasers to mitigate out-of-band phase errors caused by measuring coatings at wavelengths significantly different from their use wavelength. Finally, scanning photometers for measurements at 1,2, and $3 \omega$ were under construction to verify the coating spectral characteristics.

In addition to upgrading the coatingvendor metrology capabilities, new instruments were also required for a more deterministic process. These included a semiautomated cleaning station based on a smaller unit used at LLE for cleaning OMEGA Upgrade optics prior to coating. A similar system was also installed at Spectra-Physics. The coating chambers
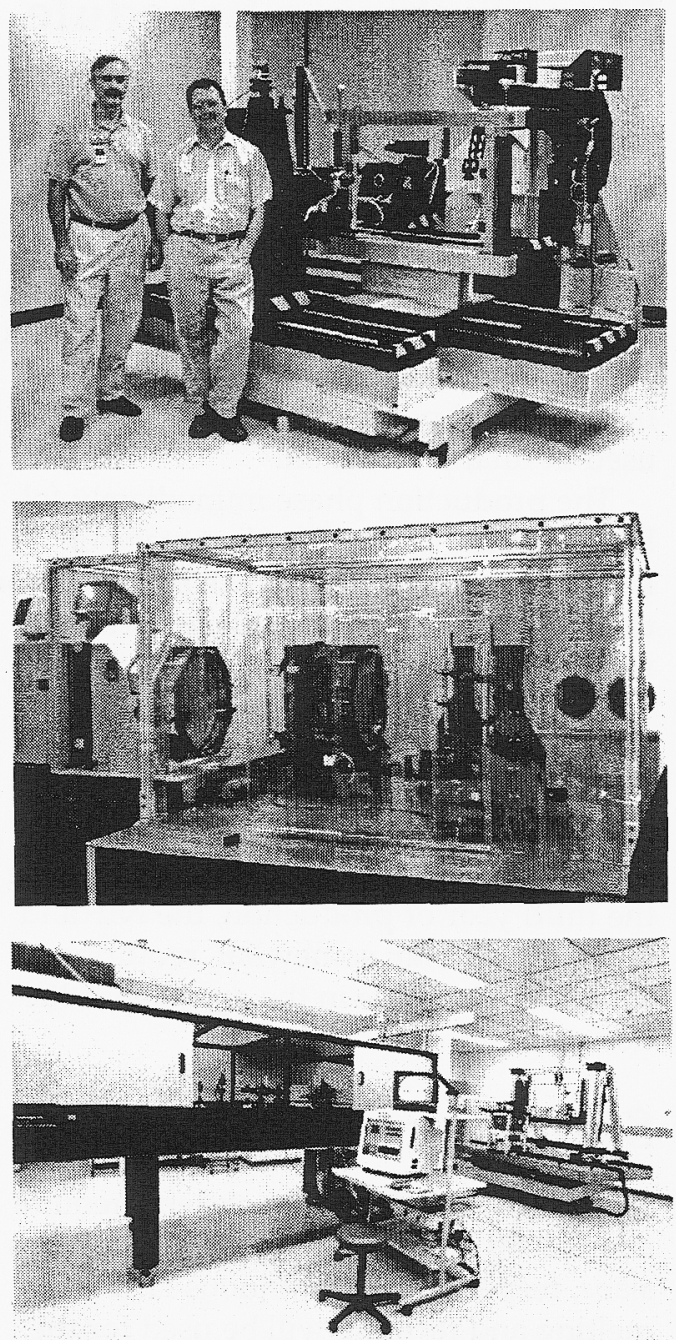

FIGURE 9. Metrology tools for characterizing NIF opticsthe photometer (top), interferometer (middle), and laserconditioning station (bottom). (40-00-0600-2885pb01) 
were upgraded with new planetaries to accommodate the larger NIF optics at LLE and for tighter performance criteria and production robustness at Spectra-Physics. Prior to coating development, thickness and refractive index errors made during the coating process were uncorrectable, resulting in poor manufacturing yields. Now in situ monitoring systems, demonstrated during facilitization and pilot, are being used for active error correction by a combination of error detection and design reoptimization. A very successful subscale demonstration of the NIF polarizer specifications occurred at both vendors, utilizing technology from coating development with equipment from facilitization.

Both Spectra-Physics and LLE began pilot activities by July 1999 . New staff were hired under the facilitization contract to field new equipment and begin process development. The same personnel are continuing in pilot efforts for process refinement.

\section{Finishing Amplifier Slabs, Mirrors, and Polarizers}

Zygo Corporation was facilitized during 1997-98 to grind, shape, clad, polish, and figure amplifier slabs, transport and cavity mirrors, and polarizers. This facilitization involved the construction and delivery of new machine tools as part of a deterministic optics fabrication process. Once the facility modifications were completed and the machine tools were installed, Zygo began pilot production on July 1, 1998. The initial phase of the pilot effort was devoted to machine tool modifications, facility improvements, and process optimization to meet the challenging NIF specifications.

In parallel, LLNL and Zygo developed the methodology for accepting finished NIF optics. This included formatting summary metrology data to be compatible with the Metrology Data Management System (MDMS), software development for analyzing wavefront files to the unique NIF specifications, and methodology for subtracting interferometric background errors. Additional pilot tasks are the documentation of test procedures and manufacturing processes, training of newly hired personnel, and factory capacity analysis. The capacity analysis combined with cost studies will be used to determine optic delivery schedules for production contracts.

In September, the first deformable and diagnostic mirrors were completed in Zygo's traditional custom optics shop. Starting in November 1999, the first pilot NIF optics from the newly facilitized NIF optics shop were completed for LLNL approval. Two specifications, microroughness and power spectral density 2 (PSD2), can only be measured at Zygo on subaperture optics because of instrument and fixture limitations. A microinterferometer at LLNL will be used to validate Zygo's process by measuring accepted full-aperture NIF optics and demonstrating that they meet the specifications. In addition, a correlation will be established between measurements on subscale witnesses and full-aperture NIF optics. In January 2000, a cumulative total of 60 NIF optics including diagnostic mirrors, deformable mirrors, elbow mirrors, transport mirrors, polarizers, and AMPLAB II amplifier slabs were accepted from Zygo.

Complete wavefront files of these optics are available for NIF performance modeling. Additionally, histograms of wavefront parameters such as peak-to-valley $(p-v)$, rms gradient, and the PSD1 roughness $(\mathrm{Rq})$ are being used to understand the impact of these specifications on manufacturing cost and NIF system performance. Histograms of these wavefront parameters in Figure 10 illustrate that the NIF specifications are being met and that the average optic is approximately $30 \%$ better than the NIF specification.

\section{Impact of Amplifier/ Mirror Finishing Pilot Yield and Technical Performance on NIF Production Costs}

The finishing manufacturing process for mirrors and polarizers consists of five major steps: shaping and edging, grinding, polish out, final figuring, and final test or metrology (Figure 11). Amplifier slabs have additional steps that are involved with cladding, which include preparing 
FIGURE 10. Histograms of $p-v, r m s$ gradient, and PSD1 Rq wavefront parameters of Zygo NIF mirrors.

(40-00-1100-6157pb01)
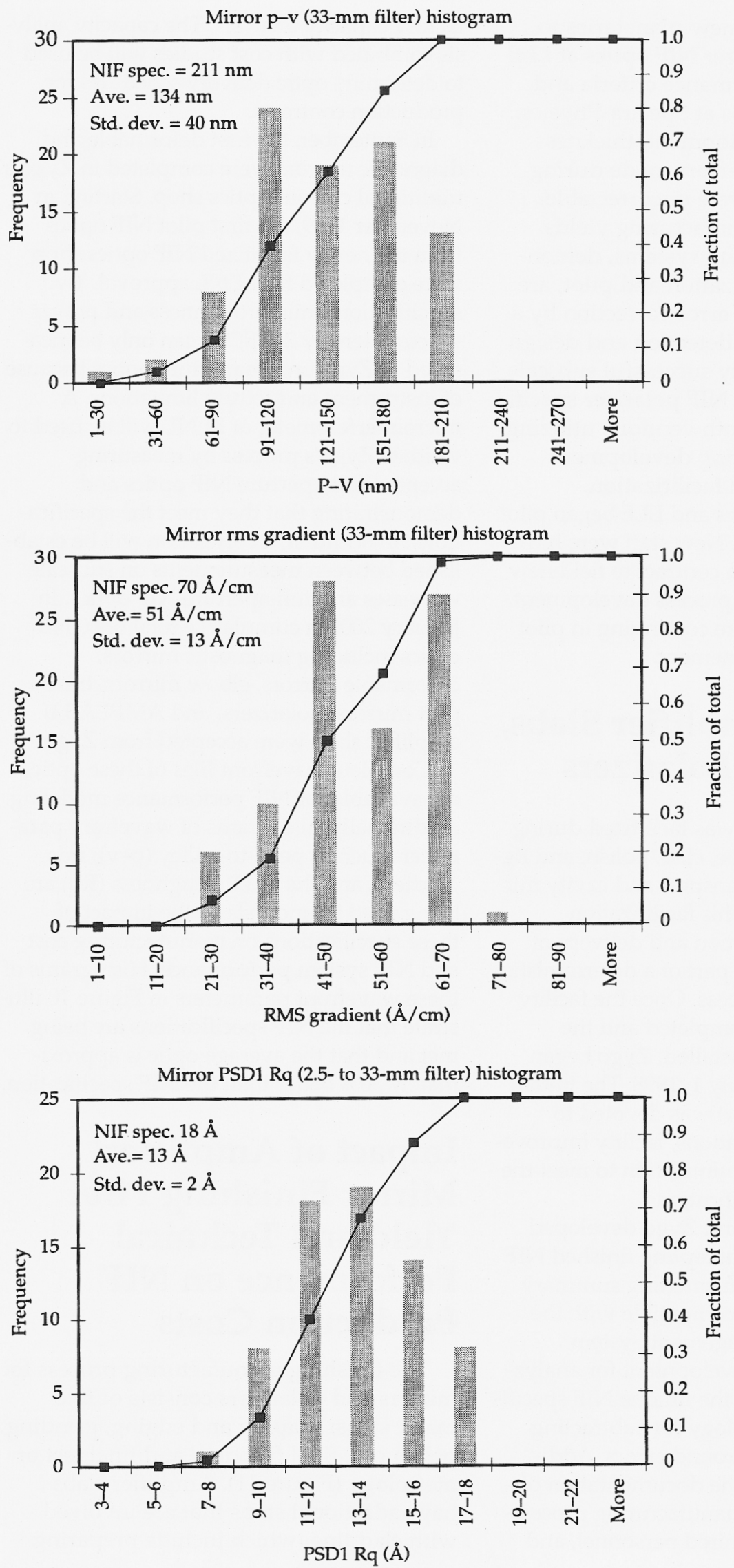


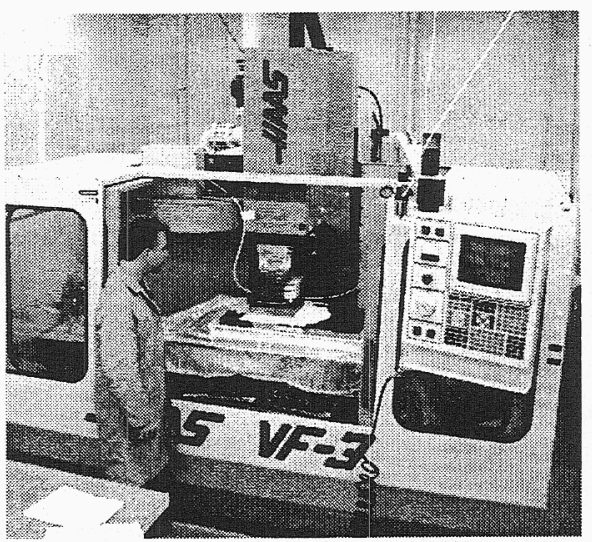

Shaping and edging

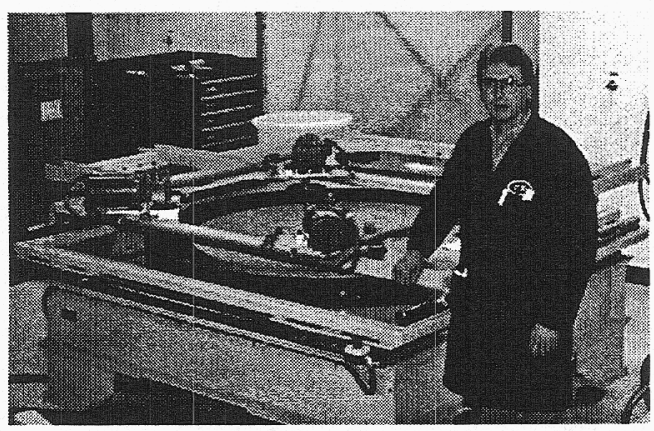

Polish out

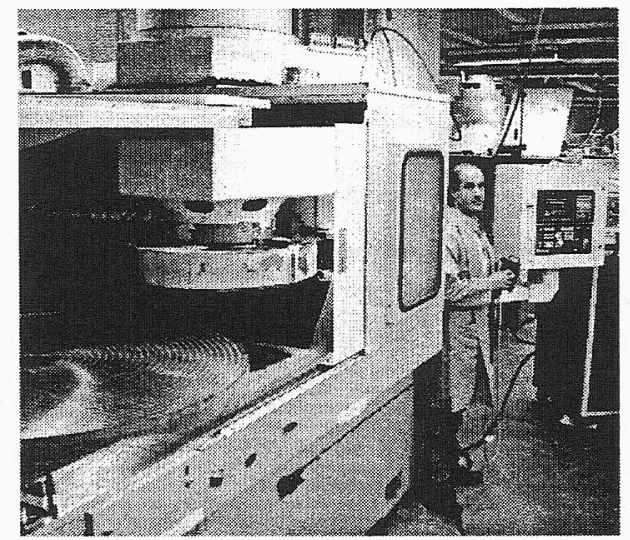

Grinding

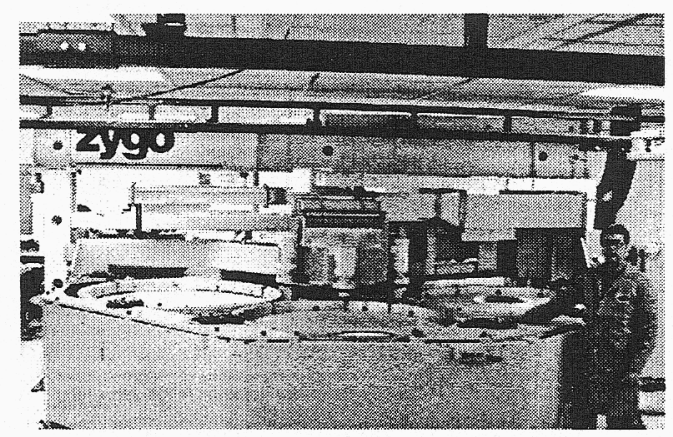

Final figuring

FIGURE 11. Five major fabrication steps for finished NIF optics-shaping and edging (top left), grinding (top right), polish out (center left), final figuring (center right), and final test (bottom). (40-00-1100-6158pb01)

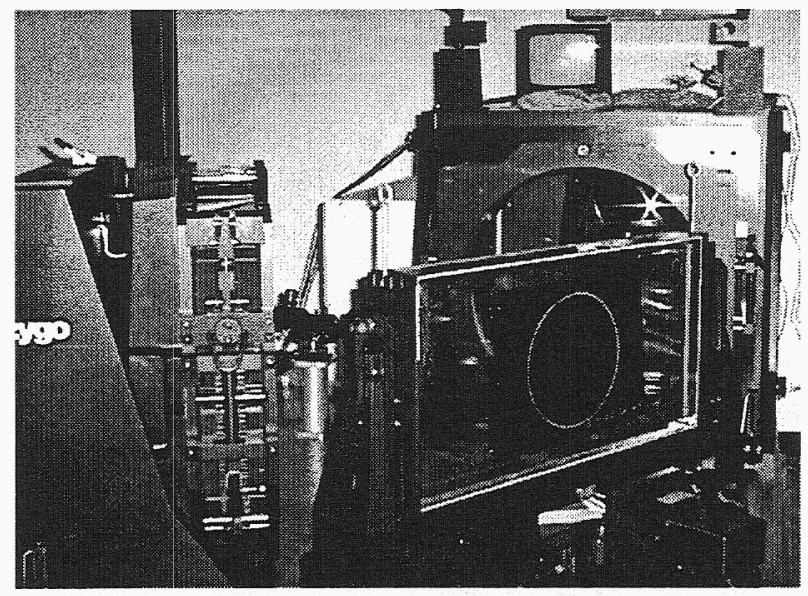

Final test

chemically the glass surfaces and epoxying the cladding strips to the laser slab. Each of these manufacturing steps uses completely different equipment and processes than those used for Nova and Beamlet to meet the aggressive cost and delivery requirements for NIF. The optics are shaped and edged on computer numerical control (CNC) fully programmable machine tools. Grinding is done using Electrolytic In-Process Dressing (ELID), a technology that has replaced the manually intensive loose-abrasive-grinding process. By running a current through the cutting tool, controlled erosion by oxidation of the surface occurs to constantly expose fresh 
sharp diamonds. Polish out occurs on a synthetic lap polisher to remove the grinding-induced surface damage and achieve nominal surface flatness. The optics are then final figured on one of three 168-in.diameter ring planetary polishers using proprietary deterministic finishing technologies. Finally, custom 24 -in.-diameter wavelength-modulating phase interferometers are used to interrogate the surface against the stringent NIF wavefront specifications.

During pilot, process demonstrations of each machine center were required to estimate the hours and hence costs of each operation. Shaping and edging by $\mathrm{CNC}$ has become routine within the optics industry; however, full-scale ELID grinding has only been done for NIF optics. During pilot, the design goal of surface flatness better than $20-\mu \mathrm{m}$ concave for both phosphate and BK7 was realized. The polish-out process produces surfaces flatter than one wave, which is $5 \times$ better than specification. The interferometers used for final test are 2 to $6 \times$ better than the NIF optic specifications. The instrument noise is $15 \times$ better than NIF optic specification. Background subtraction techniques have been formalized with custom software developed at LLNL to subtract the errors within the interferometer optics. After process optimization, final figuring has been extremely successful for BK7 optics as discussed in the previous section. Final figuring of phosphate glass is still undergoing process optimization due to the high thermal expansion of laser glass and lowerthan-desired homogeneity of the raw glass from the first pilot run. Nevertheless, a cost study was submitted by Zygo based on knowledge learned from the pilot finishing effort, which will be used as a basis for the NIF production costs.

\section{Main Lens/Window Pilot Production and Finishing of 50 First-Bundle Optics}

Lens/window pilot production began at our primary vendor, S.V.G. Tinsley, in March 1999 as an exercised option to their facilitization contract. The lens optical test system (LOTS) will be used to verify that lenses (cavity spatial filter lenses, $\mathrm{SF} 1 / 2$; transport spatial filter lenses, SF3/4; and the final focus lens, FL) conform to the NIF optical specifications. The LOTS is also used to measure the back focal length (bfl) of the lenses. During pilot production in FY99, about 30 spatial filter lenses and three focus lenses were fabricated. The bfl of a few spatial filter lenses were checked to determine if they were within the specified range using a long unequal-path interferometer. No other metrology was performed on the lenses in FY99. The LOTS is on schedule to be completed in July 2000, at which time final testing will begin for the lenses produced in FY99 as well as the 25 additional lenses made thus far in FY00.

During pilot production in FY99, 16 target chamber vacuum windows (TCVW) and four switch windows (SW) were fabricated. Testing with the metrology equipment available at Tinsley showed that the specifications for peak-to-valley and rms gradient and power spectral density 1 (PSD1) over the full aperture were met for these optics. However, when one of the SWs was delivered to LLNL and tested using the Lab's white light interferometer, it was found that the SW failed the power spectral density 2 (PSD2) but passed the microroughness specification. Further inspection at Tinsley showed that the other windows also failed PSD2. The most probable cause for the failure was determined to be small-tool ripple introduced into this spatial frequency regime. Because the flatness of the part going into their small-tool process was in excess of 5 waves, large amounts of material removal and many iterations were required in their smalltool polishing process to achieve figure. Because small-tool polishing is also used to achieve figure for lenses, a similar problem with PSD2 was observed with lenses. This problem with PSD2 on windows and the plano-side of lenses is expected to vanish when the 120-in. continuous polisher (CP), designed and built by Lapmaster, is used and the flatness before small-tool polishing is 1 wave or less.

To reduce potential risks to the NIF schedule, a small, but flexible optics fabrication facility was created at Eastman 
Kodak through a facilitization contract awarded in September of 1998. The scope of work envisioned for the flexible facility included providing postpolishing capabilities for laser glass slabs and polarizers should correction for material inhomogeneity be needed, serving as a backup vendor for finishing all $3 \omega$ fused silica optical components, and providing additional capacity for finishing primarily small transport mirrors. The Pilot Production option of the facilitization contract with Kodak was exercised in December 1998, and the production of the first of 38 prime optics began in June 1998. Due to changing needs in the NIF project, the contract was modified midway through pilot production to focus more effort on amplifiers, mirrors, and polarizers rather $3 \omega$ fused silica optics (e.g., lenses and windows).

In a separate contract with Bond Optics, Inc., five NIF-size debris shields were fabricated. Subsequent testing of these optics in FYOO using both the 24-in. and white light interferometers at LLNL showed that all the NIF optical specifications were met except for rms gradient. Up to two of these optics will undergo destructive damage testing with incident laser light at $3 \omega$ $(351 \mathrm{~nm})$. The remaining debris shields would have to be reworked before they could be used on the NIF 


\section{APPENDIX A \\ 1999 AWARDS, PATENTS, and Refereed Publications}

$\mathrm{T}$ he 1999 ICF Annual Report uses this space to report on the Awards received by,

Patents issued to, and Publications by LLNL employees in the ICF Program. The goal is to showcase the distinguished scientific and technical achievements of our employees.

The Annual Report is based on the Fiscal Year of 1999, whereas the achievements listed here are based on the Calendar Year of 1999. The reason for this disparity is the fact that a publication or award may not have a precise date other than the particular year it was given.

The awards listed are those granted by recognized organizations outside of LLNL and given to ICF Program employees.

The patents may include work that is afield from ICF but are granted to ICF Program employees. This could include work that is supported from discretionary funds.

The publications listed are those from refereed scientific journals. Therefore, conference proceedings are generally not included unless they are also published in refereed scientific journals. The work in ICF is broad-based at LLNL, and many employees who are not directly in the ICF Program publish ICF-related results; these publications are included. Furthermore, publications are included in which an ICF Program author contributes but is not the lead author. 


\title{
1999 ICF/NIF Program Awards
}

\section{American Physical Society Fellows}

Michael Key

Peter Young

\section{Teller Award}

Stephen Haan

\section{Fusion Power Associates Leadership Award \\ Grant Logan}

\author{
Otto Schott Research Award \\ Jack Campbell
}

\section{Federal Laboratory Consortium Award for "Excellence in Technology Transfer"}

Laser Shot Peening System-Lloyd Hackel, Ralph Jacobs, Curt Theisen, John Wooldridge, and Daryl Grzybicki

\section{R\&D 100 Awards}

Gamma Watermarking-Muriel Ishikawa, Lowell Wood Jr., Ron Lougheed, Kenton Moody, Winifred Parker, and Tzu Fang Wang

High-Power Diode-Pumped Solid-State Green Laser-Jim Chang, Isaac Bass, Christopher Ebbers, Ernest Dragon, and Curt Cochran

Solid-State Power Source for Advanced Accelerators and Industrial Applications-Hugh Kirbie, George Caporaso, Roy Hanks, Steve Hawkins, Craig Ollis, Brad Hickman, Bryan Lee, and Rob Saethre; Craig Brooks (Bechtel)

The Atomic Precision Multi-Layer Deposition System-James Folta, Claude Montcalm, Christopher Walton, Fred Grabner, Stephen Vernon, Gary Howe, Gary Heaton, Richard Levesque, Eberhard Spiller, Marco Wedowski, and George Wells; Mark Schmidt (Allied Signal)

\section{Society for Technical Communication Awards}

ICF Quarterly Report, Vol. 9, No. 1-Jason Carpenter, Howard Powell, Robert Kauffman, Roy Johnson, Al Miguel, Cindy Cassady, Pam Davis, Sandy Lynn, Thomas Reason, Robert Kirvel, Jeff Morris, Pat Boyd, Treva Carey, Frank Marquez, Judy Rice, Tony Sanchez, and Frank Uhlig

Target Chamber Welding photograph-Jacqueline McBride, Bryan Quintard 


\section{ICF Patents}

Lee, Northrup, Ciarlo, Krulevitch, Benett

Microfabricated Therapeutic Actuators

U.S. Patent 5,911,737

June 15, 1999

Chow, Loomis, Thomas

Optical Coatings of Variable Refractive Index and High Laser-Resistance from Physical-

Vapor-Deposited Perfluorinated Amorphous Polymer

U.S. Patent $5,882,773$

March 16, 1999

Zapata, Hackel

Lamp System with Conditioned Water Coolant and Diffuse Reflector of Polytetra-

fluorethylene (PTFE)

U.S. Patent $5,971,565$

October 26, 1999

Perry, Britten, Nguyen, Boyd, Shore

Multilayer Dielectric Diffraction Gratings

U.S. Patent 5,907,436

May 25, 1999

Fitch

Sparse Aperture Endoscope

U.S. Patent $5,919,128$

July 6, 1999

Montgomery, Zaitseva, De Yoreo, Vital

Device for Isolation of Seed Crystals During Processing of Solution

U.S. Patent $5,904,772$

May 18, 1999

Meyer, Ciarlo, Myers, Chen, Wakalopulos

Rigid Thin Windows for Vacuum Applications

U.S. Patent $6,002,202$

December 14, 1999

McEwan

Ultra-Wideband Impedance Sensor

U.S. Patent 5,883,591

March 16, 1999

Krulevitch, Lee, Northrup, Benett

Microbiopsy/Precision Cutting Devices

U.S. Patent $5,928,161$

July 27,1999

Benett, Celliers, Da Silva, Glinsky, London, Maitland, Matthews, Krulevitch, Lee Opto-Acoustic Transducer for Medical Applications

U.S. Patent $5,944,687$

August 31, 1999 
Krulevitch, Lee, Northrup, Benett

Microfabricated Instrument for Tissue Biopsy and Analysis

U.S. Patent $5,985,217$

November 16, 1999

Freitas, Skidmore

Low-Cost Laser Diode Array

U.S. Patent $5,909,458$

June 1, 1999

Lee, Krulevitch, Northrup

Micromachined Electrical Cauterizer

U.S. Patent $5,944,717$

August 31, 1999

Hsiao, Merritt, Penetrante, Vogtlin

Pre-Converted Nitric Oxide Gas in Catalytic Reduction System

U.S. Patent 5,893,267

April 6, 1999

Vogtlin, Merritt, Hsiao, Wallman, Penetrante

Catalytic Reduction System for Oxygen-Rich Exhaust

U.S. Patent 5,893,267

April 13, 1999

Chang, Bass, Zapata

Compact and Highly Efficient Laser Pump Cavity

U.S. Patent $5,978,407$

November 2, 1999

Vann

Six Degrees of Freedom Sensor

U.S. Patent $5,883,803$

March 16, 1999

Perry, Banks, Stuart, Fochs

Aberration-Free, All-Reflective Laser Pulse Stretcher

U.S. Patent $5,906,016$

September 28, 1999

Dane, Hackel

All Solid-State SBS Phase Conjugate Mirror

U.S. Patent $5,880,873$

March 9, 1999

Northrup, Yu, Raley

Porous Silicon Structures with High Surface Area/Specific Pore Size

U.S. Patent $5,882,496$

March 9, 1999

Northrup

Process for Forming a Porous Silicon Member in a Crystalline Silicon Member

U.S. Patent 6,004,450

December 21, 1999 
Montcalm, Stearns, Vernon

Passivating Overcoat Bilayer for Multilayer Reflective Coatings for Extreme Ultraviolet

Lithography

U.S. Patent $5,958,605$

September 28, 1999

Matthews, Celliers, Hackel, Da Silva, Dane, Mrowka

High Removal Rate Laser-Based Coating Removal System

U.S. Patent $5,986,234$

November 16, 1999

Skidmore, Freitas

Microlens Frames for Laser Diode Arrays

U.S. Patent 5,923,481

July 13, 1999

Da Silva, Matthews, Fitch, Everett, Colston, Stone

$X$-Ray Compass for Determining Device Orientation

U.S. Patent $5,912,945$

June 15, 1999

Wickboldt, Carey, Smith, Ellingboe

Deposition of Dopant Impurities and Pulsed Energy Drive-In

U.S. Patent 5,918,140

June 29, 1999

Carey, Smith

Method of Fabrication of Display Pixels Driven by Silicon Thin-Film Transistors

U.S. Patent $5,994,174$

November 30, 1999

Lee, Sommargren, McConaghy, Krulevitch

Micromachined Electrostatic Vertical Actuator

U.S. Patent $5,969,848$

October 19, 1999

Chapman, Hodyma, Shafer, Sweeney

Reflective Optical Imaging System with Balanced Distortion

U.S. Patent $5,973,826$

October 26, 1999

Weber, Spiller

Cleaning Process for EUV Optical Substrates

U.S. Patent $5,958,143$

September 28, 1999

Hale

Precision Tip-Tilt-Piston Actuator That Provides Exact Constraint

U.S. Patent 5,986,827

November 16, 1999 


\section{Refereed Publications}

J. J. Adams, C. Bibeau, R. H. Page, D. M. Krol, L. H. Furu, and S. A. Payne "4.0-4.5 $\mu \mathrm{m}$ Lasing of Fe:ZnSe Below $180 \mathrm{~K}$, a New Mid-Infrared Laser Material" Opt. Lett. 24(23), 1720-1722 (1999)

G. O. Allshouse, R. E. Olson, D. A. Callahan-Miller, and M. Tabak "Deposition and Drive Symmetry for Light Ion ICF Targets" Nuclear Fusion 39(7), 893-899 (1999)

K. L. Baker, R. P. Drake, K. G. Estabrook, B. Sleaford, M. K. Prasad, B. La Fontaine, and D. M. Villeneuve "Measurement of the Frequency and Spectral Width of the Langmuir Wave Spectrum Driven by Stimulated Raman Scattering"

Phys. Plasmas 6(11), 4284-4292 (1999)

P. S. Banks, L. Dinh, B. C. Stuart, M. D. Feit, A. M. Komashko, A. M. Rubenchik, M. D. Perry, and W. McLean "Short-Pulse Laser Deposition of Diamond-Like Carbon Thin Films" Appl. Phys. A 69(SUPPS), S347-S353 (1999)

P. S. Banks, M. D. Feit, A. M. Rubenchik, B. C. Stuart, and M. D. Perry "Material Effects in Ultra-Short Pulse Laser Drilling of Metals" Appl. Phys. A 69 (SUPPS), S377-S380 (1999)

P. S. Banks, M. D. Feit, and M. D. Perry

"High-Intensity Third-Harmonic Generation in Beta Barium Borate through SecondOrder and Third-Order Susceptibilities"

Opt. Lett. 24(1), 4-6 (1999)

A. J. Bayramian, C. D. Marshall, K. I. Schaffers, and S. A. Payne "Characterization of $\mathrm{Yb}^{3+}: \mathrm{Sr}_{5-\mathrm{x}} \mathrm{Ba}_{\mathrm{x}}\left(\mathrm{PO}_{4}\right)_{3} \mathrm{~F}$ Crystals for Diode-Pumped Lasers" IEEE J. Quant. Electr. 35(4), 665-674 (1999)

R. L. Berger, A. B. Langdon, J. E. Rothenberg, C. H. Still, and E. A. Williams "Stimulated Raman and Brillouin Scattering of Polarization-Smoothed and Temporally Smoothed Laser Beams"

Phys. Plasmas 6(4), 1043-1047 (1999)

D. N. Bittner, G. W. Collins, E. Monsler, and S. Letts

"Forming Uniform HD Layers in Shells Using Infrared Radiation"

Fusion Tech. 35(2), 244-249 (1999)

C. D. Boley and M. A. Rhodes

"Modeling of a Plasma Electrode Pockels Cell" IEEE Trans. Plasma Sci. 27(3), 713-726 (1999)

K. S. Budil, D. M. Gold, K. G. Estabrook, B. A. Remington, J. Kane, P. M. Bell, D. Pennington, C. Brown, S. Hatchett, J. A. Koch, M. H. Key, and M. D. Perry "Blast Wave Diagnostic for the Petawatt Laser System"

Rev. Sci. Inst. 70(1) Pt. 2, 806-809 (1999) 
D. A. Callahan-Miller and M. Tabak

"Increasing the Coupling Efficiency in a Heavy Ion, Inertial Confinement Fusion Target"

Nuclear Fusion 39(11), 1547-1556 (1999)

D. A. Callahan-Miller and M. Tabak

"A Distributed Radiator, Heavy Ion Target Driven by Gaussian Beams in a Multibeam Illumination Geometry"

Nuclear Fusion 39(7), 883-891 (1999)

R. Cauble, B. A. Remington, and E. M. Campbell

"Laboratory Measurements of Materials in Extreme Conditions: The Use of High Energy Radiation Sources for High Pressure Studies"

J. Impact Engr. 23(1) Pt. 1, 87-99 (1999)

H. L. Chen, D. Francis, T. Nguyen, W. P. Yuen, G. Li, and C. Chang-Hasnain

"Collimating Diode Laser Beams from a Large-Area VCSEL-Array Using

Microlens Array"

IEEE Phot. Tech. Lett. 11(5), 506-508 (1999)

C. Cherfils, S. G. Glendinning, D. Galmiche, B. A. Remington, A. L. Richard, S. Haan,

R. Wallace, N. Dague, and D. H. Kalantar

"Convergent Rayleigh-Taylor Experiments on the Nova Laser"

Phys. Rev. Lett. 83 (26), 5507-5510 (1999)

B. I. Cohen, E. B. Hooper, T. B. Kaiser, E. A. Williams, and C. W. Domier

"Modeling of Ultra-Short-Pulse Reflectometry"

Phys. Plasmas 6(5), 1732-1741 (1999)

G. W. Collins, P. M. Celliers, D. M. Gold, L. B. Da Silva, and R. Cauble "Shock-Compression Experiments and Reflectivity Measurements in Deuterium up to 3.5 Mbar Using the Nova Laser"

Cont. to Plasma Phys. 39(N1-2), 13-16 (1999)

R. C. Cook, R. L. McEachern, and R. B. Stephens

"Representative Surface Profile Power Spectra from Capsules Used in Nova and Omega Implosion Experiments"

Fusion Tech. 35(2), 224-228 (1999)

R. Cook, S. R. Buckley, E. Fearon, and S. A. Letts

"New Approaches to the Preparation of P $\alpha$ MS Beads as Mandrels for NIF-Scale Target

Capsules"

Fusion Tech. 35(2), 206-211 (1999)

S. N. Crichton, M. Tomozawa, J. S. Hayden, T. I. Suratwala, and J. H. Campbell "Subcritical Crack Growth in a Phosphate Laser Glass"

J. Am. Ceramic. Soc. 82(11), 3097-3104 (1999)

G. Dimonte

"Nonlinear Evolution of the Rayleigh-Taylor and Richtmyer-Meshkov Instabilities"

Phys. Plasmas 6(5) Pt. 2, 2009-2015 (1999) 
T. Ditmire, J. Zweiback, V. P. Yanovsky, T. E. Cowan, G. Hays, and K. B. Wharton "Nuclear Fusion from Explosions of Femtosecond Laser-Heated Deuterium Clusters" Nature 398(6727), 489-492 (1999)

T. R. Dittrich, S. W. Haan, M. M. Marinak, S. M. Pollaine, D. E. Hinkel, D. H. Munro, C. P. Verdon, G. L. Strobel, R. McEachern, R. C. Cook, C. C. Roberts, D. C. Wilson, P. A. Bradley, L. R. Foreman, and W. S. Varnum

"Review of Indirect-Drive Ignition Design Options for the National Ignition Facility" Phys. Plasmas 6(5) Pt. 2, 2164-2170 (1999)

P. F. Dubois

"Scientific Components Are Coming"

IEEE Computer 32(3), 115-117 (1999)

D. R. Farley, K. G. Estabrook, S. G. Glendinning, S. H. Glenzer, B. A. Remington, K. Shigemori, J. M. Stone, R. J. Wallace, G. B. Zimmerman, and J. A. Harte "Radiative Jet Experiments of Astrophysical Interest Using Intense Lasers" Phys. Rev. Lett. 83(10), 1982-1985 (1999)

M. Fleischhauer, R. Unanyan, B. W. Shore, and K. Bergmann "Coherent Population Transfer Beyond the Adiabatic Limit: Generalized Matched Pulses and Higher-Order Trapping States"

Phys. Rev. A 59(5), 3751-3760 (1999)

S. G. Glendinning, P. Amendt, B. D. Cline, R. B. Ehrlich, B. A. Hammel, D. H. Kalantar, O. L. Landen, R. E. Turner, R. J. Wallace, T. J. Weiland, N. Dague, J.-P. Jadaud, D. K. Bradley, G. Pien, and S. Morse "Hohlraum Symmetry Measurements with Surrogate Solid Targets" Rev. Sci. Inst. 70(1), 536-542 (1999)

S. H. Glenzer, W. E. Alley, K. G. Estabrook, J. S. De Groot, M. G. Haines, J. H. Hammer, J.-P. Jadaud, B. J. MacGowan, J. D. Moody, W. Rozmus, L. J. Suter, T. L. Weiland, and E. A. Williams

"Thomson Scattering from Laser Plasmas"

Phys. Plasmas 6(5) Pt. 2, 2117-2128 (1999)

S. H. Glenzer, W. Rozmus, B. J. MacGowan, K. G. Estabrook, J. D. De Groot, G. B. Zimmerman, H. A. Baldis, B. A. Hammel, J. A. Harte, R. W. Lee, E. A. Williams, and B. G. Wilson

"Thomson Scattering from High-Z Laser-Produced Plasmas"

Phys. Rev. Lett. 82(1), 97-100 (1999).

P. M. Gresho

"Some Aspects of the Hydrodynamics of the Microencapsulation Route to NIF Mandrels"

Fusion Tech. 35(2), 157-188 (1999)

I. Haber, A. Friedman, D. P. Grote, S. M. Lund, and R. A. Kishek

"Recent Progress in the Simulation of Heavy Ion Beams"

Phys. Plasmas 6(5) Pt. 2, 2254-2261 (1999)

J. Hammer and D. D. Ryutov "Linear Stability of an Accelerated, Current Carrying Wire Array" Phys. Plasmas 6(8), 3302-3315 (1999) 
J. H. Hammer, M. Tabak, S. C. Wilks, J. D. Lindl, D. S. Bailey, P. W. Rambo, A. Toor, G. B. Zimmerman, and J. L. Porter, Jr.

"High Yield Inertial Confinement Fusion Target Design for a Z-Pinch Driven Hohlraum"

Phys. Plasmas 6(5) Pt. 2, 2129-2136 (1999)

F. V. Hartemann, E. C. Landahl, A. L. Troha, J. R. Van Meter, H. A. Baldis, R. R. Freeman, N. C. Luhman, L. Song, A. K. Kerman, and D. U. L. Yu

"The Chirped-Pulse Inverse Free-Electron Laser: A High-Gradient Vacuum Laser

Accelerator"

Phys. Plasmas 6(10), 4104-4110 (1999)

D. E. Hinkel, R. L. Berger, E. A. Williams, A. B. Langdon, C. H. Still, and B. F. Lasinski "Stimulated Brillouin Backscatter in the Presence of Transverse Plasma Flow"

Phys. Plasmas 6(2), 571-581 (1999)

\section{R. A. Hyde}

"Eyeglass 1. Very Large Aperture Diffractive Telescopes"

Appl. Opt. 38(19), 4198-4212 (1999)

D. H. Kalantar, B. A. Remington, E. A Chandler, J. D. Colvin, D. M. Gold, K. O. Mikaelian, S. V. Weber, L. G. Wiley, J. S. Wark, A. A. Hauer, and M. A. Meyers

"High Pressure Solid State Experiments on the Nova Laser"

J. Impact Engr. 23(1) Pt. 1, 409-419 (1999)

D. H. Kalantar, E. A. Chandler, J. D. Colvin, R. Lee, B. A. Remington, S. V. Weber,

L. G. Wiley, A. Hauer, J. S. Wark, A. Loveridge, B. H. Failor, M. A. Meyers, and

G. Ravichandran

"Transient X-Ray Diffraction Used to Diagnose Shock Compressed Si Crystals on the

Nova Laser"

Rev. Sci. Inst. 70(1), 629-632 (1999)

J. Kane, D. Arnett, B. A. Remington, S. G. Glendinning, S. G. Bazan, R. P. Drake, B. A. Fryxell, R. Teyssier, and K. Moore

"Scaling Supernova Hydrodynamics to the Laboratory"

Phys. Plasmas 6(5) Pt. 2, 2065-2071 (1999)

J. Kane, R. P. Drake, and B. A. Remington

"An Evaluation of the Richtmyer-Meshkov Instability in Supernova Remnant Formation" Astrophys. J. 511(N1PT1), 335-340 (1999)

J. D. Kilkenny, T. P. Bernat, B. A. Hammel, R. L. Kauffman, O. L. Landen, J. D. Lindl, B. J. MacGowan, J. A. Paisner, and H. T. Powell

"Lawrence Livermore National Laboratory's Activities to Achieve Ignition by X-Ray

Drive on the National Ignition Facility"

Laser and Part. Beams 17(2), 159-171 (1999)

J. D. Kilkenny, E. M. Campbell, J. D. Lindl, G. B. Logan, W. R. Meier, L. J. Perkins, J. A. Paisner, M. H. Key, H. T. Powell, R. L. McCrory, and W. Seka

"The Role of the National Ignition Facility in Energy Production from Inertial Fusion"

Phil. Trans. Royal Soc. of London 357(1752), 533-553 (1999)

R. K. Kirkwood, D. S. Montgomery, B. B. Afeyan, J. D. Moody, B. J. MacGowan, C. Joshi, K. B. Wharton, S. H. Glenzer, E. A. Williams, P. E. Young, W. L. Kruer, K. G. Estabrook, and R. L. Berger 
"Observation of the Nonlinear Saturation of Langmuir Waves Driven by Ponderomotive Force in a Large Scale Plasma"

Phys. Rev. Lett. 83(15), 2965-2968 (1999)

J. A. Koch, O. L. Landen, B. A. Hammel, C. Brown, J. Seely, and Y. Aglitskiy

"Recent Progress in High-Energy, High-Resolution X-Ray Imaging Techniques for Application to the National Ignition Facility"

Rev. Sci. Inst. 70(1), 525-529 (1999)

R. Kodama, K. Takahashi, K. A. Tanaka, Y. Kato, K. Murai, F. Weber, T. W. Barbee, and L. B. Da Silva

"Measurements of Laser-Hole Boring into Overdense Plasmas Using X-Ray Laser Refractometry"

Rev. Sci. inst. 70(1) 543-548 (1999)

A. M. Komashko, M. D. Feit, A. M. Rubenchik, M. D. Perry, and P. S. Banks "Simulation of Material Removal Efficiency with Ultrashort Laser Pulses"

Appl. Phys. A 69(SUPPS), S95-S98 (1999)

W. L. Kruer, E. M. Campbell, C. D. Decker, S. C. Wilks, J. Moody, T. Orzechowski, L. Powers, L. J. Suter, B. B. Afeyan, and N. Dague

"Strongly Driven Laser-Plasma Coupling"

Plasma Phys. E Contr. Fusion 41(Supp3A), A409-A417 (1999)

C. Labaune, H. A. Baldis, B. Cohen, W. Rozmus, S. Depierreux, E. Schifano, B. S. Bauer, and A. Michard

"Nonlinear Modification of Laser-Plasma Interaction Processes under Crossed Laser Beams"

Phys. Plasmas 6(5) Pt. 2, 2048-2056 (1999)

C. Labaune, H. A. Baldis, B. S. Bauer, E. Schifano, and B. I. Cohen

"Spatial and Temporal Coexistence of Stimulated Scattering Processes Under CrossedLaser-Beam Irradiation"

Phys. Rev. Lett. 82(18), 3613-3616 (1999)

E. C. Landahl, F. V. Hartemann, G. P. Le Sage, W. E. White, H. A. Baldis, C. V. Bennnett, J. P. Heritage, B. H. Kolner, N. C. Luhman, and C. H. Ho

"Phase Noise Reduction and Photoelectron Acceleration in a High-Q RF Gun" IEEE Trans. Plasma Sci. 27(5), 1547 (1999)

O. L. Landen, P. A. Amendt, L. J. Suter, R. E. Turner, S. G. Glendinning, S. W. Haan, S. M. Pollaine, B. A. Hammel, M. Tabak, M. D. Rosen, and J. D. Lindl "A Simple Time-Dependent Analytic Model of the $\mathrm{P}_{2}$ Asymmetry in Cylindrical Hohlraums"

Phys. Plasmas 6(5) Pt. 2, 2137-2143 (1999)

B. F. Lasinski, A. B. Langdon, S. P. Hatchett, M. H. Key, and M. Tabak "Particle in Cell Simulations of Ultra Intense Laser Pulses Propagating Through Overdense Plasma for Fast-Ignitor and Radiography Applications"

Phys. Plasmas 6(5) Pt. 2, 2041-2047 (1999)

J. F. Latkowski, J. J. Sanchez, and L. C. Pittenger "Neutronics and Activation Analysis for the National Ignition Facility Cryogenic Target Positioner"

Fusion Tech. 35(2), 255-259 (1999) 
R. J. Leeper, T. E. Alberts, J. R. Asay, P. M. Baca, K. L. Baker, S. P. Breeze, G. A. Chandler, D. L. Cook, G. W. Cooper, C. Deeney, M. S. Derzon, M. R. Douglas, D. L. Fehl,

T. Gilliland, D. E. Hebron, M. J. Hurst, D. O. Jobe, J. W. Kellogg, J. S. Lash, S. E. Lazier, M. K. Matzen, D. H. McDaniel, J. S. McGurn, T. A. Mehlhorn, A. R. Moats, R. C. Mock, D. J. Muron, T. J. Nash, R. E. Olson, J. L. Porter, J. P. Quintenz, P. V. Reyes, L. S. Ruggles, C. L. Ruiz, T. W. L. Sanford, F. A. Schmidlapp, J. F. Seamen, R. B. Spielman, M. A. Stark, K. W. Struve, W. A. Stygar, D. R. Tibbetts-Russell, J. A. Torres, T. Vargas, T. C. Wagoner, C. Wakefield, J. H. Hammer, D. D. Ryutov, M. Tabak, S. C. Wilks, R. L. Bowers, K. D. McLenithan, and D. L. Peterson

"Z Pinch Driven Inertial Confinement Fusion Target Physics Research at Sandia National Laboratories"

Nuclear Fusion 39(9Y 1), 1283-1294 (1999)

R. A. Lerche and T. J. Ognibene

"Error Analysis for Fast Scintillator-Based Inertial Confinement Fusion Burn History Measurements"

Rev. Sci. Inst. 70(1), 1217-1219 (1999)

B. G. Logan, M. D. Perry, and G. J. Caporaso

"Concept for High-Charge-State Ion Induction Accelerators"

Fusion Engr. and Design 44, 295-301 (1999)

R. McEachern and C. Alford

"Evaluation of Boron-Doped Beryllium as an Ablator for NIF Target Capsules"

Fusion Tech. 35(2), 115-118 (1999)

J. H. McGuire, A. L. Godunov, S. G. Tolmanov, H. Schmidt-Bocking, R. Dorner, V. Mergel, R. Dreizler, and B. W. Shore

"Time Ordering in Fast Double Injection"

Intl J. Mass Spect. 192(SI), 65-73 (1999)

D. S. Montgomery, R. P. Johnson, J. A. Cobble, J. C. Fernandez, E. L. Lindman, H. A. Rose, and K. G. Estabrook

"Characterization of Plasma and Laser Conditions for Single Hot Spot Experiments"

Laser and Particle Beams 17(3), 349-359 (1999)

J. D. Moody, B. J. MacGowan, S. H. Glenzer, R. K. Kirkwood, W. L. Kruer, A. J. Schmitt, E. A. Williams, and G. F. Stone

"First Measurement of Short Length-Scale Density Fluctuations in a Large Laser Plasma" Phys. Rev. Lett. 83(9), 1783-1786 (1999)

J. D. Moody, B. J. MacGowan, S. H. Glenzer, R. K. Kirkwood, W. L. Kruer, S. M. Pollaine, E. A. Williams, G. F. Stone, B. B. Afeyan, and A. J. Schmitt

"Measurements of Near Forward Scattered Laser Light in a Large Inertial Confinement Fusion Plasma"

Rev. Sci. Inst. 70(1), 677-681 (1999)

M. J. Moran

"The Fusion Diagnostic Gamma Experiment: A High-Bandwidth Fusion Diagnostic of the National Ignition Facility"

Rev. Sci. Inst. 70(1) 1226-1228 (1999)

S. Nakai and B. G. Logan

"Strategy Toward Inertial Fusion Energy"

Fusion Engr. \& Design 44, 97-104 (1999) 
T. J. Nash, M. S. Derzon, G. A. Chandler, R. Leeper, D. Fehl, J. Lash, C. Ruiz, G. Cooper, J. F. Seaman, J. McGurn, S. Lazier, J. Torres, D. Jobe, T. Gilliland, M. Hurst, R. Mock, P. Ryan, D. Nielsen, J. Armijo, J. McKenney, R. Hawn, D. Hebron, J. J. MacFarlane, D. Petersen, R. Bowers, W. Matuska, and D. D. Ryutov "High-Temperature Dynamic Hohlraums on the Pulsed Power Driver Z" Phys. Plasmas 6(5) Pt. 2, 2023-2029 (1999)

T. Norimatsu, Y. Izawa, K. Mima, and P. M. Gresho "Modeling of the Centering Force in a Compound Emulsion to Make Uniform Plastic Shells for Laser Fusion Targets"

Fusion Tech 35(2), 147-156 (1999)

M. C. Nostrand, R. H. Page, S. A. Payne, W. F. Krupke, and P. G. Schunemann "Room-Temperature Laser Action at 4.3-4.4 $\mathrm{mm}$ in $\mathrm{CaGa}_{2} \mathrm{~S}_{4}: \mathrm{Dy}^{3+"}$

Opt. Lett. 24(17), 1215-1217 (1999)

L. J. Perkins

"Antiproton Fast Ignition for Inertial Confinement Fusion"

Fus. Tech. 36(4), 219-233 (1999)

M. D. Perry, B. C. Stuart, P. S. Banks, M. D. Feit, V. Yanovsky, and A. M. Rubenchik "Ultrashort-Pulse Laser Machining of Dielectric Materials"

J. Appl. Phys. 85(9), 6803-6810 (1999)

M. D. Perry, D. Pennington, B, C. Stuart, G. Tietbohl, J. A. Britten, C. Brown, S. Herman, B. Golick, M. Kartz, J. Miller, H. T. Powell, M. Vergino, and V. Yanovsky

"Petawatt Laser Pulses"

Opt. Lett. 24(3), 160-162 (1999)

M. D. Perry, J. A. Sefcik, T. Cowan, S. Hatchett, A. Hunt, M. Moran, D. Pennington, R. Snavely, and S. C. Wilks

"Hard X-Ray Production from High.Intensity Laser Solid Interactions"

Rev. Sci. Inst. 70(1), 265-279 (1998)

T. W. Phillips, M. D. Cauble, T. E. Cowan, S. P. Hatchett, E. A. Henry, M. H. Key, M. D. Perry, T. C. Sangster, and M. A. Stoyer

"Diagnosing Hot Electron Production by Short Pulse, High Intensity Lasers Using Photonuclear Reactions"

Rev. Sci. Inst. 70(1), 1213-1216 (1999)

B. A. Remington, D. Arnett, R. P. Drake, and H. Takabe

"Modeling Astrophysical Phenomena in the Laboratory with Intense Lasers"

Science 284, 1488-1493 (1999)

C. C. Roberts, S. A. Letts, M. Saculla, E. J. Hsieh, and R. C. Cook

"Polyimide Films from Vapor Deposition: Toward High Strength, NIF Capsules"

Fusion Tech. 35(2), 138-146 (1999)

M. D. Rosen

"The Physics Issues That Determine Inertial Confinement Fusion Target Gain and Driver Requirements: A Tutorial"

Phys. Plasmas 6(5) Pt. 2, 1690-1699 (1999) 
D. D. Ryutov, R. P. Drake, J. Kane, E. Liang, B. A. Remington, and W. M. Wood-Vasey "Similarity Criteria for the Laboratory Simulations of Supernova Hydrodynamics" Astrophys. J. 518(N2PT1), 821-832 (1999)

D. D. Ryutov

"Landau Damping: Half a Century with a Great Discovery"

Plasma Phys. and Controlled Fusion 4(3A), A1-A12 (1999)

V. Sankaran, M. J. Everett, D. J. Maitland, and J. T. Walsh

"Comparison of Polarized-Light Propagation in Biological Tissue and Phantoms"

Opt. Lett. 24(15), 1044-1046 (1999)

V. Sankaran, K. Schonenberger, J. T. Walsh, Jr., and D. J. Maitland

"Polarization Discrimination of Coherently Propagating Light in Turbid Media"

Appl. Opt. 38(19), 4252-4261 (1999)

J. Sater, B. Kozioziemski, G. W. Collins, E. R. Mapoles, J. Pipes, J. Burmann, and T. P. Bernat "Cryogenic D-T Fuel Layers Formed in $1 \mathrm{~mm}$ Spheres by Beta-Layering"

Fusion Tech. 35(2), 229-233 (1999)

U. S. Sathyam, B. W. Colston, Jr., L. B. Da Silva, and M. J. Everett

"Evaluation of Optical Coherence Quantitation of Analytes in Turbid Media by Use of Two Wavelengths"

Appl. Opt. 38(10), 2097-2104 (1999)

A. I. Shestakov

"Time Dependent Simulations of Point Explosions with Heat Conduction"

Phys. Plasmas 11(5), 1091-1095 (1999)

L. J. Suter, O. L. Landen, and J. I. Koch

"Prospects for Fluorescence Based Imaging/Visualization of Hydrodynamic Systems on the National Ignition Facility"

Rev. Sci. Inst. 70(1) 663-666 (1999)

R. E. Turner, O. L. Landen, P. Bell, R. Costa, and D. Hargrove

“Achromatically Filtered Diamond Photoconductive Detectors for High Power Soft X-Ray Flux Measurements"

Rev. Sci. Inst. 70(1), 656-658 (1999)

R. G. Unanyan, B. W. Shore, and K. Bergmann

"Laser-Driven Population Transfer in Four-Level Atoms: Consequences of Non-Abelian Geometrical Adiabatic Phase Factors"

Phys. Rev. A 59(4), 2910-2919 (1999)

N. V. Vitanov, K. A. Suominen, and B. W. Shore

"Creation of Coherent Atomic Superpositions by Fractional Stimulated Raman Adiabatic Passage"

J. Phys. B 32(18), 4535-4546 (1999)

G. J. Wagner, T. J. Carrig, R. H. Page, K. I. Schaffers, J. O. Ndap, X. Y. Ma, and A. Burger "Continuous-Wave Broadly Tunable $\mathrm{Cr}^{2+}: \mathrm{ZnSe}$ Laser"

Opt. Lett. 24(1), 19-21 (1999) 
J. M. Wallace, T. J. Murphy, N. D. Delamater, K. A. Klare, J. A. Oertel, G. R. Magelssen, E. L. Lindman, A. A. Hauer, P. Gobby, J. D. Schnittman, R. S. Craxton, W. Seka, R. Kremens, D. Bradley, S. M. Pollaine, R. E. Turner, O. L. Landen, D. Drake, and J. J. MacFarlane "Inertial Confinement Fusion with Tetrahedral Hohlraums at OMEGA"

Phys. Rev. Lett. 82(19), 3807-3810 (1999)

K. B. Wharton, R. K. Kirkwood, S. H. Glenzer, K. G. Estabrook, B. B. Afeyan, B. I. Cohen, J. D. Moody, B. J. MacGowan, and C. Joshi

"Observation of Resonant Energy Transfer between Identical-Frequency Laser Beams" Phys. Plasmas 6(5) Pt. 2, 2144-2149 (1999)

L. P. Yatsenko, B. W. Shore, T. Halfmann, K. Bergmann, and A. Vardi "Source of Metastable H(2s) Atoms Using the Stark Chirped Rapid-Adiabatic-Passage Technique"

Phys. Rev. A 60(6), R4237-4240 (1999)

L. P. Yatsenko, T. Halfmann, B. W. Shore, and K. Bergmann

"Photoionization Suppression by Continuum Coherence: Experiment and Theory" Phys. Rev. A 59(4), 2926-2947 (1999)

N. Zaitseva, J. Atherton, R. Rozsa, L. Carman, I. Smolsky, M. Runkel, R. Ryon, and L. James "Design and Benefits of Continuous Filtration in Rapid Growth of Large KDP and DKDP Crystals"

J. Crystal Growth 197(4), 911-920 (1999)

F. Ze, O. L. Landen, P. M. Bell, R. E. Turner, T. Tutt, S. S. Alvarez, and R. L. Costa "Investigation of Quantum Efficiencies in Multilayered Photocathodes for Microchannel Plate Applications"

Rev. Sci. Inst. 70(1), 659-662 (1999)

J. Zweiback, T. Ditmire, and M. D. Perry

"Femtosecond Time-Resolved Studies of the Dynamics of Noble-Gas Cluster Explosions" Phys. Rev. A 59(5), R3166-R3169 (1999) 


\section{APPENDIX B}

\section{ICF QUARTERLY REPORT ARTICLES IN SEQUENCE}

\begin{tabular}{|c|c|c|c|}
\hline AUTHOR & ARTICLE & VoL./No. & PAGE \\
\hline J. K. Crane & The NIF Injection Laser System & $(9) 1$ & 1 \\
\hline M. Newton & Main Amplifier Power Conditioning for the NIF & (9) 1 & 15 \\
\hline P. J. VanArsdall & Integrated Computer Control System & (9)1 & 21 \\
\hline M. Rhodes & Multiaperture Optical Switch for the NIF & $(9) 1$ & 33 \\
\hline P. Wegner & Beamlet Experiments & (9) 1 & 43 \\
\hline M. D. Feit & $\begin{array}{l}\text { Modeling the Interaction of the NIF Laser Beam } \\
\text { with Laser Components }\end{array}$ & (9) 1 & 63 \\
\hline E. S. Bliss & Beam Control and Laser Diagnostic Systems & (9) 1 & 79 \\
\hline A. C. Erlandson & $\begin{array}{l}\text { Design and Performance of Flashlamp-Pumped } \\
\text { Nd:Glass Amplifiers for the NIF }\end{array}$ & (9) 1 & 99 \\
\hline J. H. Campbell & $\begin{array}{l}\text { Properties of and Manufacturing Methods } \\
\text { for NIF Laser Glasses }\end{array}$ & (9)2 & 111 \\
\hline J. A. Britten & Diffractive Optics for the NIF & (9)2 & 125 \\
\hline A. K. Burnham & $\begin{array}{l}\text { Producing KDP and DKDP Crystals for the } \\
\text { NIF Laser }\end{array}$ & (9)2 & 135 \\
\hline C. J. Stolz & $\begin{array}{l}\text { Engineering High-Damage-Threshold NIF } \\
\text { Polarizers and Mirrors }\end{array}$ & (9) 2 & 151 \\
\hline P. K. Whitman & Improved Antireflection Coatings for the NIF & $(9) 2$ & 163 \\
\hline T. G. Parham & $\begin{array}{l}\text { Developing Optics Finishing Technologies for the } \\
\text { National Ignition Facility }\end{array}$ & $(9) 2$ & 177 \\
\hline M. R. Kozlowski & $\begin{array}{l}\text { Laser-Damage Testing and Modeling Methods } \\
\text { for Predicting the Performance of Large-Area } \\
\text { NIF Optics }\end{array}$ & $(9) 2$ & 193 \\
\hline A. K. Burnham & $\begin{array}{l}\text { Development of the NIF Target Chamber First } \\
\text { Wall and Beam Dumps }\end{array}$ & $(9) 2$ & 203 \\
\hline D. C. Eder & $\begin{array}{l}\text { Control of Debris and Shrapnel Generation in the } \\
\text { National Ignition Facility }\end{array}$ & $(9) 3$ & 213 \\
\hline
\end{tabular}


AUTHOR

O. L. Landen ARTICLE

X-Ray Backlighting for the National Ignition Facility

B. A. Remington Using Intense Lasers in the Laboratory to Model Astrophysical Phenomena

C. A. Back

Radiation Transport Experiments on the OMEGA Laser

J. Edwards

A New Laser-Driven Source for Studying

Radiation Hydrodynamic Phenomena

Vol./No. PAGE

(9) $3 \quad 227$

(9)3 239

(9)3 255

(9)3 263 


\section{APPENDIX C \\ Publications and Presentations OCTOBER 1998-SEPTEMBER 1999}

A

Afeyan, B. B., Fleischer, J. W., Geddes, C., Montgomery, D. S., Kirkwood, R., Estabrook, K., Schmitt, A. J., Meyerhofer, D., and Town, R. P. J., Optical Mixing Controlled Stimulated Scattering Instabilities: Effects of a Large Amplitude Probe Beam on the Backscattering of a Pump Beam, Lawrence Livermore National Laboratory, Livermore, CA, UCRL-JC-133943-ABS. Prepared for 29th Annual Anomalous Absorption Conf, Pacific Grove, CA, Jun 13, 1999.

Afeyan, B. B., Geddes, C., Montgomery, D., Kirkwood, R., Meyerhofer, D., and Schmitt, A., Optical Mixing Controlled Stimulated Scattering Instabilities (OMCSSI) Experiments on the NIF, Lawrence Livermore National Laboratory, Livermore, CA, UCRL-JC-135258 ABS. Prepared for Frontier Science at the NIF: Episode I, Pleasanton, CA, Oct 4, 1999.

Albrecht, G. F., Sutton, S. B., George, E. V., Sooy, W. R., and Krupke, W. F., Solid State Heat Capacity Disk Laser, Lawrence Livermore National Laboratory, Livermore, CA, UCRL-JC-130963; also in Laser \& Part. Beams 16(4), 605-625 (1998).

Allshouse, G. O., Olson, R. E., CallahanMiller, D. A., and Tabak, M., Deposition and Drive Symmetry for Light Ion ICF Targets, Lawrence Livermore National Laboratory, Livermore, CA, UCRL-JC-131395; also in Nuclear Fusion 39(7), 893-899 (1999).
Amendt, P. A., Estabrook, K., Everett, M., London, R. A., Maitland, D., Zimmerman, G., Colston, B., and Sathyam, U., Monte Carlo Simulations of Arterial Imaging with Optical-Coherence-Tomography, Lawrence Livermore National Laboratory, Livermore, CA, UCRL-JC-135024 ABS. Prepared for Photonic West 2000 Symp, San Jose, CA, Jan 23, 2000.

Amendt, P. A., Turner, R. E., Bradley, D., Landen, O., Haan, S., Suter, L. J., Wallace, R., Morse, S., Pien, G., and Seka, W., HighConvergence Indirect-Drive Implosions on OMEGA: Design and Simulations, Lawrence Livermore National Laboratory, Livermore, CA, UCRL-JC-130677 ABS Rev. Prepared for 41st Annual Mtg of the American Physical Society Div of Plasma Physics, Seattle, WA, Nov 15, 1999.

Amendt, P. A., Turner, R. E., Landen, O. L., Bradley, D. K., Suter, L. J., McEachern, R., Wallace, R. J., and Hammel, B. A., High Growth Factor, High Convergence Implosions in Cylindrical Hohlraums, Lawrence Livermore National Laboratory, Livermore, CA, UCRL-JC-133631-ABS. Prepared for 1st Intl Conf on Inertial Fusion Sciences and Applications, Bordeaux, France, Sept 12, 1999. 
Amendt, P., Turner, R. E., Landen, O. L., Decker, C., Glendinning, S. G., Haan, S. W., Jones, O. S., Pollaine, S. M., Suter, L. J., and Wallace, R., High-Convergence Implosion Studies on the OMEGA Laser with Indirectly-Driven Vacuum Hohlraums, Lawrence Livermore National Laboratory, Livermore, CA, UCRL-JC-133890-ABS. Prepared for 29th Annual Anomalous Absorption Conf, Pacific Grove, CA, Jun 13, 1999.

Arabadzhi, V. V., Bogatyrev, S. D., Druzhinin, O. A., Kazakov, V. I., Korotkov, D. P., Matusov, P. A., Pugatchev, V. A., Serin, B. V., Talanov, V. I., and Zaborskikh, D. V., Experimental Study of Internal Waves Generated by a Sphere Moving in a Stratified Fluid with a Thermocline, Lawrence Livermore National Laboratory, Livermore, CA, UCRL-JC-123583. Submitted to J. Fluid Mech.

Atherton, J., Inertial Confinement Fusion Quarterly Report, January-March 1999, Volume 9, Number 2, Lawrence Livermore National Laboratory, Livermore, CA, UCRL-LR-105821-99-2.

\section{B}

Back, C. A., Alvarez, S., Spragge, M. Bauer, J., Landen, O. L., Turner, R. E., and Hsing, W., High Energy Density Experimental Science in Support of SBSS, Lawrence Livermore National Laboratory, Livermore, CA, UCRL-JC-131975 ABS. Prepared for Women's Technical and Professional Symp, San Ramon, CA, Oct 15, 1998.

Back, C. A., Bauer, J. D., Landen, O. L., Turner, R. E., Lasinski, B. F., Hammer, J. H., Rosen, M. D., Suter, L. J., and Hsing, W. H., Detailed Measurements of a Diffusive Supersonic Wave in a Radiatively Heated Foam, Lawrence Livermore National Laboratory, Livermore, CA, UCRL-JC135062. Submitted to Phys. Rev. Lett.
Back, C. A., Bauer, J. D., Turner, R. E., Lasinski, B. F., Hammer, J., Suter, L. J., Landen, O. L., and Hsing, W. W., Uses of $X$-Ray Emission to Study Radiative Processes in Laser-Produced Plasmas, Lawrence Livermore National Laboratory, Livermore, CA, UCRL-JC-135560 ABS. Prepared for Frontier Science at the NIF: Episode I, Pleasanton, CA, Oct 4, 1999.

Back, C. A., Diffusive, Supersonic X-Ray Transport in Foam Cylinders, Lawrence Livermore National Laboratory, Livermore, CA, UCRL-JC-134467 ABS Rev. Prepared for 41st Annual Mtg of the American Physical Society Div of Plasma Physics, Seattle, WA, Nov 15, 1999.

Back, C. A., Landen, O. L., Turner, R. E., Koch, J. A., Hammel, B. A., MacFarlane, J. J., and Nash, T. J., Spectroscopic Measurements to Investigate and Diagnose Radiative Heating of Hohlraum Walls, Lawrence Livermore National Laboratory, Livermore, CA, UCRL-JC-131993 ABS.

Prepared for 8th Intl Workshop on Radiative Properties of Hot Dense Matter, Sarasota, FL, Oct 26, 1998.

Banks, P. S., Feit, M. D., and Perry, M. D., High-Intensity Third-Harmonic Generation in Beta Barium Borate through Second-Order and Third-Order Susceptibilities, Lawrence Livermore National Laboratory, Livermore, CA, UCRL-JC-131446; also in Opt. Lett. 24(1), 4-6 (1999).

Barton, I. M., Dixit, S., Summers, L. J., Thompson, C. A., Avicola, K., and Wilhelmsen, J., Diffractive Alvarez Lens, Lawrence Livermore National Laboratory, Livermore, CA, UCRL-JC-135583.

Submitted to Opt. Lett.

Battersby, C. L., Sheehan, L. M., and Kozlowski, M. R., Effects of Wet Etch Processing on Laser-Induced Damage of Fused Silica Surfaces, Lawrence Livermore National Laboratory, Livermore, CA, UCRL-JC-131224. Prepared for 30th Boulder Damage Symp: Annual Symp on Optical Materials for High Power Lasers, Boulder, CO, Sept 28, 1998. 
Belian, A.P., Latkowski, J. F., and Morse, E. C., "Experimental Studies of Concrete Activation at the National Ignition Facility Using the Rotating Target Neutron Source," Fusion Tech. 34(3), Pt. 2, 1028-1032 (1998).

Benjamin, D. W., Construction Safety Program for the National Ignition Facility, July 30, 1999, Lawrence Livermore National Laboratory, Livermore, CA, UCRL-ID-125990 Rev 2.

Berger, R. L., Cohen, B. I., Langdon, A. B., MacGowan, B. J., Rothenberg, J., Still, C. W., Williams, E. A., and Lefebvre, E., Simulation of the Nonlinear Interaction of Filamentation with Stimulated Backscatter, Lawrence Livermore National Laboratory, Livermore, CA, UCRL-JC-132598 ABS. Prepared for 1999 Centennial Mtg of the American Physical Society, Atlanta, GA, Mar 20, 1999.

Berger, R. L., Langdon, A. B., Hinkel, D. E., Still, C. H., and Williams, E. A., Evolution of the Frequency and Wavenumber of the Transmitted and Reflected Light in $3 D$ Simulations, Lawrence Livermore National Laboratory, Livermore, CA, UCRL-JC133777-ABS. Prepared for 29th Annual Anomalous Absorption Conf, Pacific Grove, CA, Jun 13, 1999.

Berger, R. L., Langdon, A. B, Rothenberg, J. E., Still, C. H., Williams, E. A., and Lefebvre, E., Stimulated Raman and Brillouin Scattering of Polarization Smoothed and Temporally Smoothed Laser Beams, Lawrence Livermore National Laboratory, Livermore, CA, UCRL-JC-132259; also in Phys. of Plasmas 6(4), 1043-1047 (1999).

Berger, R. L., Moody, J. D., Langdon, A. B., Hinkel, D. E., Still, C. H., and Williams, E. A., Influence of Nonlocal Electron Transport on Stimulated Forward Brillouin Scattering and Filamentation, Lawrence Livermore National Laboratory, Livermore, CA, UCRL-JC-134902 ABS. Prepared for 41st Annual Mtg of the American Physical Society Div of Plasma Physics, Seattle, WA, Nov 15, 1999.
Berger, R. L., Still, C. H., Williams, E. A., and Langdon, A. B., On the Dominant and Subdominant Behavior of Stimulated Raman and Brillouin Scattering Driven by

Nonuniform Laser Beams, Lawrence

Livermore National Laboratory,

Livermore, CA, UCRL-JC-132259; also in Phys. Plasmas 5(12), 4337-4356 (1998).

Bernat, T., Target Technologies for Ignition on the NIF, Lawrence Livermore National Laboratory, Livermore, CA, UCRL-JC135691. Prepared for 1st Intl Conf on Inertial Fusion Sciences and Applications, Bordeaux, France, Sep 12, 1999.

Besenbruch, G. E., Alexander, N. B., Baugh, W. A., Bernat, T. P., Collins, R. P., Boline, K. K., Brown, L. C., Gibson, C. R., Goodin, D. T., and Harding, D. R., Design and Testing of Cryogenic Target Systems, Lawrence Livermore National Laboratory, Livermore, CA, UCRL-JC-135692.

Prepared for 1st Intl Conf on Inertial Fusion Sciences and Applications, Bordeaux, France, Sep 12, 1999.

Bibeau, C., Beach, R. J., Mitchell, S. C., Emanuel, M. A., Skidmore, J., Ebbers, C. A., Sutton, S. B., and Jancaitis, K. S., High Average Power $1 \mathrm{~mm}$ Performance and Frequency Conversion of a Diode-End-Pumped $Y$ Y:YAG Laser, Lawrence Livermore National Laboratory, Livermore, CA, UCRL-JC-129612; also in IEEE J. Quant. Electr. 34(10), 2010-2019 (1998).

Bittner, D. N., Collins, G. W., Monsler, E., and Letts, S., Forming Uniform HD Layers in Shells Using Infrared Radiation, Lawrence Livermore National Laboratory, Livermore, CA, UCRL-JC-131371; also in Fusion Tech. 35(2), 244-249 (1999).

Bittner, D., Burmann, J., Collins, G., Lee, Y., Mackinnon, A., and Unites, W., IR Layering in a Hohlraum, Lawrence Livermore National Laboratory, Livermore, CA, UCRL-JC-135500 ABS. Prepared for Target Fabrication Mtg '99, Catalina Island, CA, Nov 8, 1999.

Boege, S. J., and Bliss, E. S., Beam Control and Laser Characterization for NIF, Lawrence Livermore National Laboratory, Livermore, CA, UCRL-JC-129854; also in Fusion Tech. 34(3), Pt. 2, 1117-1121 (1998). 
Boley, C. D., and Rhodes, M. A., Modeling of a Plasma Electrode Pockels Cell, Lawrence Livermore National Laboratory, Livermore, CA, UCRL-JC-131445; also in IEEE Trans. Plasma Sci. 27(3), 713-726 (1999).

Bradley, D. K., Pollaine, S., Glendinning, G., Landen, O., Turner, R., Amendt, P. A., and Wallace, R., Measurements of P6 and P8 Modes in NIF-Scale Hohlraums Using Point Projection Radiography, Lawrence Livermore National Laboratory, Livermore, CA, UCRL-JC-134959 ABS. Prepared for 41st Mtg of the American Physical Society Div of Plasma Physics, Seattle, WA, Nov 15, 1999.

Branham, K. E., Mays, J. W., Gray, G. M., Cook, R., and Byrd, H., Preparation of Soluble, Linear Titanium-Containing Copolymers by the Free Radical Copolymerization of Vinyl Titanate Monomers with Styrene, Lawrence Livermore National Laboratory, Livermore, CA, UCRL-JC-134776. Submitted to J. of Applied Polymer Sciences.

Brereton, S. J., Latkowski, J., Singh, M., Tobin, M., and Yatabe, J., Decommissioning Plan for the National Ignition Facility, Lawrence Livermore National Laboratory, Livermore, CA, UCRL-JC-130859; also in Fusion Tech. 34(3), Pt. 2, 1041-1046 (1998).

Brereton, S. J., Yatabe, J. M., and Taylor, C. A., The Safety and Environmental Process for the Design and Construction of the National Ignition Facility, Lawrence Livermore National Laboratory, Livermore, CA, UCRL-JC-130860; also in Fusion Tech. 34(3), Pt. 2, 760-766 (1998).

Brereton, S., Ma, C., Newton, M., Pastrnak, J., Price, D., and Prokosch, D., Pressure Effects Analysis of National Ignition Facility Capacitor Module Events, Lawrence Livermore National Laboratory, Livermore, CA, UCRL-JC-135684 ABS. Prepared for 8th Intl Conf on Nuclear Engineering, Baltimore, MD, Apr 2, 2000.
Britten, J. A., and Summers, L. J., Multiscale, Multifunction Diffractive Structures Wet-Etched into Fused Silica for High-Laser Damage Threshold Applications, Lawrence Livermore National Laboratory, Livermore, CA, UCRL-JC-130115; also in Appl. Optics 37(30), 7049-7054 (1998).

Britten, J. A., Herman, S. M., Summers, L. J., Rushford, M. C., Auyang, L., Barton, L. M., Shore, B. W., Dixit, S. N., Parham, T. G., and Hoaglan, C. R., Manufacture, Optical Performance and Laser Damage Characteristics of Diffractive Optics for the National Ignition Facility, Lawrence Livermore National Laboratory, Livermore, CA, UCRL-JC-131517. Prepared for 30th Boulder Damage Symp: Annual Symp on Optical Materials for High Power Lasers, Boulder, CO, Sept 28, 1998.

Budil, K. S., Edwards, M. J., Lasinski, B., Remington, B. A., Suter, L., Wan, A. S., and Stry, P. E., Ablation Front Rayleigh-Taylor Experiments at Nova, Lawrence Livermore National Laboratory, Livermore, CA, UCRL-JC-135063 ABS. Prepared for 41st Annual Mtg of the American Physical Society Div of Plasma Physics, Seattle, WA, Nov 15, 1999.

Budil, K. S., Gold, D. M., Estabrook, K. G., Remington, B. A., Kane, J., Bell, P. M., Pennington, D., Brown, C., Hatchett, S., Koch, J. A., Key, M. H., and Perry, M. D., Blast Wave Diagnostic for the Petawatt Laser System, Lawrence Livermore National Laboratory, Livermore, CA, UCRL-JC130955; also in Rev. Sci. Inst. 70(1) Pt. 2, 806-809 (1999).

Budil, K. S., Remington, B. A., Glendinning, S. G., Kalantar, D. H., Robey, H. F., Farley, D. R., Shigemori, K., Perry, T. S., Louis, H., and Demiris, A., Diagnostic Techniques for Hydrodynamics Experiments on Intense Lasers, Lawrence Livermore National Laboratory, Livermore, CA, UCRL-JC-135602 ABS. Prepared for Frontier Science at the NIF: Episode I, Pleasanton, CA, Oct 4, 1999. 
Bullock, A. B., Dimonte, G., Hsing, W. W., Dunne, M., Graham, P., Thomas, B., Barnes, C. W., and Goldman, S. R., Quantitative Comparison of Experimental and Simulations Images, Lawrence Livermore National Laboratory, Livermore, CA, UCRL-JC-135061 ABS. Prepared for 41st Annual Mtg of the American Physical Society Div of Plasma Physics, Seattle, WA, Nov 15, 1999.

Burmann, J., Sanchez, J., and Pipes, J., NIF Scale Cryogenic Hohlraum Assembly $\mathcal{E}$ Testing Techniques, Lawrence Livermore National Laboratory, Livermore, CA, UCRL-JC-135543 ABS. Prepared for Target Fabrication $\mathrm{Mtg}$ '99, Catalina Island, CA, Nov 8, 1999.

\section{C}

Callahan-Miller, D. A., and Tabak, M., A Distributed Radiator, Heavy Ion Target Driven by Gaussian Beams in a Multibeam Illumination Geometry, Lawrence Livermore National Laboratory, Livermore, CA, UCRL-JC-131543; also in Nuclear Fusion 39(7), 883-891 (1999).

Chang, L., Lean Manufacture of Assemblies for the National Ignition Facility, Lawrence Livermore National Laboratory, Livermore, CA, UCRL-JC-134230-ABS. Prepared for 3rd Biennial Tri-Laboratory Engineering Conf on Modeling and Simulation, Pleasanton, CA, Nov 3, 1999.

Chatterjee, I., Hallock, G. A., and Wootton, A. J., Observation of Multiple Modes of Interior Density Turbulence in the TEXT-U Tokamak, Lawrence Livermore National Laboratory, Livermore, CA, UCRL-JC132555.

Cherfils, C., Glendinning, S. G., Galmiche, D., Remington, B. A., Richard, A. L., Hann, S., Wallace, R., Dague, N., and Kalantar, D. H., Convergent Rayleigh-Taylor Experiments on the Nova Laser, Lawrence Livermore National Laboratory, Livermore, CA, UCRL-JC-135371. Submitted to Phys. Rev. Lett.
Collins, G. W., Celliers, P. M., Gold, D. M., Da Silva, L. B., and Cauble, R., ShockCompression Experiments and Reflectivity Measurements in Deuterium up to $3.5 \mathrm{Mbar}$ Using the Nova Laser, Lawrence Livermore National Laboratory, Livermore, CA, UCRL-JC-132119; also in Cont. to Plasma Physics 39(1-2), 13-16 (1999).

Colvin, J. D., Amendt, P. A., Dittrich, T. R., Tipton, R. E., and Vantine, H. C., Analysis of Laser Indirect Drive Double-Shell Capsule Implosion Experiments as a Testbed for Benchmarking Compressible Turbulent Mix Models, Lawrence Livermore National Laboratory, Livermore, CA, UCRL-JC132571 ABS. Prepared for 1999 Centennial Mtg of the American Physical Society, Atlanta, GA, Mar 20, 1999.

Cook, B., Takagi, M., Buckley, S., Fearon, E., and Hassel, A., Mandrel Development Update-1/98 to 12/98, Lawrence Livermore National Laboratory, Livermore, CA, UCRL-ID-133144 (1999).

Cook, R. C., McEachern, R. L., and Stephens, R. B., Representative Surface Profile Power Spectra from Capsules Used in Nova and Omega Implosion Experiments, Lawrence Livermore National Laboratory, Livermore, CA, UCRL-JC-129961; also in Fusion Tech. 35(2), 224-228 (1999).

Cook, R. C., Models of Polyimide Spray Coatings, Lawrence Livermore National Laboratory, Livermore, CA, UCRL-JC135542 ABS. Prepared for Target Fabrication Mtg '99, Catalina Island, CA, Nov 8, 1999.

Cook, R., Buckley, S. R., Fearon, E., and Letts, S. A., New Approaches to the Preparation of PoMS Beads as Mandrels for NIF-Scale Target Capsules, Lawrence Livermore National Laboratory, Livermore, CA, UCRL-JC-129955; also in Fusion Tech. 35(2), 206-211 (1999).

Cooke, D. W., Muenchausen, R. E., and Bennett, B. L., Measurement of Point Defect Energetics in Potassium Dihydrogen Phosphate (KDP), Lawrence Livermore National Laboratory, Livermore, CA, UCRL-CR-133032. 
D

De Yoreo, J. J., Yan, M., Runkel, M., Staggs, M., Liou, L., Demos, S. G., Carman, L., Zaitseva, N. P., and Rek, Z. U., Characterization of Optical Performance and Defect Structures in Nonlinear Crystals for Large Aperture Lasers, Lawrence Livermore National Laboratory, Livermore, CA, UCRL-JC-132557 ABS-S. Prepared for Conf on Lasers and Electro-Optics '99/Quantum Electronics and Laser Science Conf'99, Baltimore, MD, May 23, 1999.

De Yoreo, J., Land, T. A., Orm, C., Teng, $\mathrm{H}$. H., McBride, M. T., Martin, T., Dove, P. M., Tayhas, G., and Palmore, T. P., Force Microscopy Investigation of Trends in Surface Dynamics during Crystal Growth from Solutions, Lawrence Livermore National Laboratory, Livermore, CA, UCRL-JC133041-ABS. Prepared for Union of Crystallographers General Assembly and Intl Congress of Crystallography, Glasgow, Scotland, Aug 4, 1999.

Decker, C., Berger, R. L., Moody, J., Williams, E. A., Suter, L. S., and Lours, L., Designs of $\mathrm{He} / \mathrm{H}_{2}$ Filled Gasbag Experiments on Nova, Lawrence Livermore National Laboratory, Livermore, CA, UCRL-JC134331-ABS. Prepared for 29th Annual Anomalous Absorption Conf, Pacific Grove, CA, Jun 13, 1999.

Decker, C., Suter, L., Back, C., Serduke, F., Grun, J., Laming, M., and Davis, J., Soft XRay Sources Using Laser Driven Metallic Vapor Targets, Lawrence Livermore National Laboratory, Livermore, CA, UCRL-JC-135023 ABS. Prepared for 41st Annual Mtg of the American Physical Society Div of Plasma Physics, Seattle, WA, Nov 15, 1999.

Dimonte, G., and Schneider, M., Density Ratio Dependence of Rayleigh-Taylor Mixing for Sustained and Impulsive Acceleration Histories, Lawrence Livermore National Laboratory, Livermore, CA, UCRL-JC132570. Submitted to Phys. of Plasmas.
Dimonte, G., New Buoyancy-Drag Model for Rayleigh-Taylor Mixing and Comparison with Experiments, Lawrence Livermore National Laboratory, Livermore, CA, UCRL-JC132695 ABS. Prepared for 7 th Intl Workshop on the Physics of Compressible Turbulent Mixing, St. Petersburg, Russia, Jul 5, 1999.

Dimonte, G., Nonlinear Evolution of the Rayleigh-Taylor and Richtmyer-Meshkov Instabilities, Lawrence Livermore National Laboratory, Livermore, CA, UCRL-JC132439; also in Phys. Plasmas 6(5), Pt.2, 2009-2015 (1999).

Dimonte, G., Spanwise Homogeneous Buoyancy-Drag Model for Rayleigh-Taylor Mixing and Comparison with Experiments, Lawrence Livermore National Laboratory, Livermore, CA, UCRL-JC-133936.

Submitted to Phys. of Plasmas.

Ditmire, T., Zweiback, J., Yanovsky, V. P., Cowan, T. E., Hays, G., and Wharton, K. B., Nuclear Fusion from Explosions of Femtosecond Laser-Heated Deuterium Clusters, Lawrence Livermore National Laboratory, Livermore, CA, UCRL-JC135040; also in Nature 398(6727), 489-492 (1999).

Dittrich, T. R., Amendt, P. A., Turner, R. E., Landen, O. L., Vantine, H. C., Tipton, R. E., and Verdon, C. P., Pushered Single Shell Capsule Implosion Experiments on OMEGA, Lawrence Livermore National Laboratory, Livermore, CA, UCRL-JC-134781 ABS Rev.

Prepared for 41st Annual Mtg of the American Physical Society Div of Plasma Physics, Seattle, WA, Nov 15, 1999.

Dittrich, T. R., Haan, S. W., Marinak, M. M., and Pollaine, S. M., Alternate Ablator Materials for NIF Ignition Capsules, Lawrence Livermore National Laboratory, Livermore, CA, UCRL-JC-134934 ABS Rev. Prepared for Target Fabrication Mtg '99, Catalina Island, CA, Nov 8, 1999.

Dittrich, T. R., Haan, S. W., Marinak, M. M., Pollaine, S. M., and McEachern, R., Reduced Scale National Ignition Fusion Capsule Design, Lawrence Livermore National Laboratory, Livermore, CA, UCRL-JC-131383; also in Phys. Plasmas 5(10), 3708-3713 (1998). 
Dittrich, T. R., Haan, S. W., Marinak, M. M., Pollaine, S. M., Hinkel, D. E., Munro, D. H., Verdon, C. P., Strobel, G. L., McEachern, R., Cook, R. C., Roberts, C. C., Wilson, D. C., Bradley, P. A., Foreman, L. R., and Varnum, W. S., Review of IndirectDrive Ignition Design Options for the National Ignition Facility, Lawrence Livermore National Laboratory, Livermore, CA, UCRL-JC-131383; also in Phys. Plasmas 6(5), Pt. 2, 2164--2170 (1999).

Dubois, P. F., "Scientific Components Are Coming," IEEE Computer 32(3), 115-117 (1999).

\section{$\mathbf{E}$}

Eder, D. C., Pretzler, G., Fill, E., Eidmann, K., and Saemann, A., Spatial Characteristics of $\mathrm{K} \alpha$ Radiation from Weakly Relativistic Laser Plasmas, Lawrence Livermore National Laboratory, Livermore, CA, UCRL-JC-133236. Submitted to Applied Phys. $B$.

Edwards, M. J., Glendinning, S. G., Budil, K. S., Lasinski, B., Shepard, T. D., Stry, P. E., Suter, L. J., Remington, B. A., Wan, A., and Wallace, R. J., Hydrodynamics of Radiation-Driven Thin Foils, Lawrence Livermore National Laboratory, Livermore, CA, UCRL-JC-135435 ABS. Prepared for Joint Operations Weapons Operations Group 37, Aldermaston, UK, Sep 20, 1999.

Edwards, M. J., Turbulent Hydrodynamic Experiments Using a New Plasma Piston, Lawrence Livermore National Laboratory, Livermore, CA, UCRL-JC-135022 ABS. Prepared for 41st Annual Mig of the American Physical Society Div of Plasma Physics, Seattle, WA, Nov 15, 1999.

Erlandson, A. C., Jancaitis, K., Marshall, C., Pedrotti, L., Zapata, L., Rotter, M., Sutton, S., Payne, S., Seznec, S., and Beullier, J., Recent Advances in FlashlampPumped Neodymium-Glass Amplifiers, Lawrence Livermore National Laboratory, Livermore, CA, UCRL-JC-132461 ABS-S. Prepared for Conf on Lasers, and Electro Optics/Quantum Electronics and Lasers Science (CLEO/QELS '99), Baltimore, MD, May 23, 1999.
Erlandson, A, Alger, T., Horvath, J., Jancaitis, K., Lawson, J., Manes, K., Marshall, C., Moor, E., Payne, S., Pedrotti, L., Rodriguez, S., Rotter, M., Sutton, S., Zapata, L., Seznec, S., Beullier, J., Carbourdin, O., Grebot, E., Guenet, I., Guenet, M., LeTouze, G., and Maille, X., "Flashlamp-Pumped Nd:Glass Amplifiers for the National Ignition Facility," Fusion Tech. 34(3), Pt. 2, 1105-1112 (1998).

Estabrook, K. G., Remington, B. A., Farley, D., Glenzer, S., Back, C. A., Turner, R. E., Glendinning, S. G., Zimmerman, G. B., Harte, J. H., and Wallace, R. J., Nova Laser Experiments of Radiative Astrophysical Jets, Lawrence Livermore National Laboratory, Livermore, CA, UCRL-JC-132258 ABS.

Prepared for 193rd Mtg of the American Astronomical Society, Austin, TX, Jan 5, 1999.

Everett, M., Kozioziemski, B., and Schafer, J. S., Novel Method to Measure Layer

Thickness, Lawrence Livermore National Laboratory, Livermore, CA, UCRL-JC135498 ABS. Prepared for Target Fabrication Mtg '99, Catalina Island, CA, Nov 8, 1999.

\section{F}

Farley, D. R., Estabrook, K. G., Glendinning, S. G., Glenzer, S. H., Remington, B. A., and Stone, J. M., Radiative Jet of Astrophysical Interest Produced at the Nova Laser Facility, Lawrence Livermore National Laboratory, Livermore, CA, UCRL-JC-133046-DR. Submitted to Phys. Rev. Lett.

Farley, D. R., Estabrook, K. G., Glendinning, S. G., Glenzer, S. H., Remington, B. A., Shigemori, K., Stone, J. M., Wallace, R. J., Zimmerman, G. B., and Harte, J. A., Radiative Jet Experiments of Astrophysical Interest Using Intense Lasers, Lawrence Livermore National Laboratory, Livermore, CA, UCRL-JC-134287; also in Phys. Rev. Lett. 83(10), 1982-1985 (1999). 
Farley, D., Remington, B., Estabrook, K., Glendinning, G., Back, C., Zimmerman, G., Harte, J., Turner, B., Glenzer, S., and Wallace, R., Nova Simulations of Astrophysical Jets, Lawrence Livermore National Laboratory, Livermore, CA, UCRL-JC-132904-COM. Submitted to New Scientist.

Fearon, E., Characterization of Russian Ballistic Furnace Shells, February, 1999, Batch, Lawrence Livermore National Laboratory, Livermore, CA, UCRL-ID133929.

Feit, M. D., Rubenchik, A. M., Kozlowski, M. R., Genin, F. Y., Schwartz, S., and Sheehan, L. M., Extrapolation of Damage Test Data to Predict Performance of LargeArea NIF Optics at $355 \mathrm{~nm}$, Lawrence Livermore National Laboratory, Livermore, CA, UCRL-JC-131222. Prepared for 30th Boulder Damage Symp: Annual Symp on Optical Materials for High Power Lasers, Boulder, CO, Sept 28, 1998.

Fill, E., Pretzler, G., Eder, D. C., Eidmann, K., and Saemann, A., Relativistic Electron Beam Interaction and $k \alpha$-Generation in Solid Targets, Lawrence Livermore National Laboratory, Livermore, CA, UCRL-JC134846. Prepared for 44th Annual Mtg of the Intl Symp on Optical Science, Engineering, and Instrumentation, Denver, CO, Jul 18, 1999.

\section{G}

Galmiche, D., Cherfils, C., Glendinning, S. G., Laffite, S., Remington, B. A., Richard, A., and Wallace, R. J., Numerical Analysis of Spherically Convergent Rayleigh-Taylor Experiments on the Nova Laser, Lawrence Livermore National Laboratory, Livermore, CA, UCRL-JC-135438. Prepared for 1st Intl Conf on Inertial Fusion Sciences and Applications, Bordeaux, France, Sep 12, 1999.
Geddes, C. G. R., Afeyan, B. B., Fleischer, J. W., Kirkwood, R. K., Montgomery, D. S., Meyerhofer, D., Seka, W., Estabrook, K., Schmitt, A. J., and Town, R. P. J., Backscatter and Transmitted Beam Measurements at OMEGA for Optical Mixing and Other Experiments, Lawrence Livermore National Laboratory, Livermore, CA, UCRL-JC-133941-ABS. Prepared for 29th Annual Anomalous Absorption Conf, Pacific Grove, CA, Jun 13, 1999.

Geddes, C. G., Kirkwood, R. K., Glenzer, S. H., Estabrook, K. G., Joshi, C., and Wharton, K. B., Study of the Saturation of Stimulated Raman Scattering by Secondary Decays, Lawrence Livermore National Laboratory, Livermore, CA, UCRL-JC130328 ABS Rev 3. Prepared for 41st Annual Mtg of the American Physical Society Div of Plasma Physics, Seattle, WA, Nov 15, 1999.

Giedt, W., and Sanchez, J., Natural Convection Heat Transfer in a NIF Capsule, Lawrence Livermore National Laboratory, Livermore, CA, UCRL-JC-135592 ABS.

Prepared for Target Fabrication Mtg '99, Catalina Island, CA, Nov 8, 1999.

Glendinning, S. G., Ablation Front Rayleigh-Taylor Growth Experiments in Spherically Convergent Geometry, Lawrence Livermore National Laboratory, Livermore, CA, UCRL-JC-134966 ABS. Prepared for 41st Annual Mtg of the American Physical Society Div of Plasma Physics, Seattle, WA, Nov 15, 1999.

Glendinning, S. G., Amendt, P., Cline, B. D., Ehrlich, R. B., Hammel, B. A., Kalantar, D. H., Landen, O. L., Turner, R. E., Wallace, R. J., Weiland, T. J., Dague, N., Jadaud, J.-P., Bradley, D. K., Pien, G., and Morse, S., Hohlraum Symmetry Measurements with Surrogate Solid Targets, Lawrence Livermore National Laboratory, Livermore, CA, UCRL-JC-129773; also in Rev. Sc. Instrum. 70(1), 536-542 (1999). 
Glendinning, S. G., Edwards, M. J., Moreno, J., Bradley, D., Hsing, W. W., Peyser, T., Remington, B. A., and Turano, E., Laser Driven Shock Tube Experiments: Direct vs. Indirect Drive, Lawrence Livermore National Laboratory, Livermore, CA, UCRL-JC-135437 ABS. Prepared for Joint Operations Weapons Operations Group 37, Aldermaston, UK, Sep 20, 1999.

Glendinning, S. G., Laser-Drive ICF Fundamentals and Recent Experimental Results, Lawrence Livermore National Laboratory, Livermore, CA, UCRL-JC132158 ABS. Prepared for Women's Technical and Professional Symp, San Ramon, CA, Oct 15, 1998.

Glendinning, S. G., Remington, B. A., Wallace, R. J., Galmiche, D., Cherfils, C., Holstein, P. A., and Richard, A., Ablative Rayleigh-Taylor Instability in Spherically Convergent Geometry, Lawrence Livermore National Laboratory, Livermore, CA, UCRL-JC-133828-ABS. Prepared for 29th Annual Anomalous Absorption Conf, Pacific Grove, CA, Jun 13, 1999.

Glenzer, S. H., Alley, W. E., Estabrook, K. G., De Groot, J. S., Haines, M. G., Hammer, J. H., Jadaud, J.-P., MacGowan, B. J., Moody, J. D., Rozmus, W., Suter, L. J., Weiland, T. L., and Williams, E. A., Thomson Scattering from Laser Plasmas, Lawrence Livermore National Laboratory, Livermore, CA, UCRL-JC-131357; also in Phys. Plasmas 6(5), Pt. 2, 2117-2128 (1999).

Glenzer, S. H., Estabrook, K. G., Lee, R. W., MacGowan, B. J., and Rozmus, W., Detailed Characterization of Laser Plasmas for Benchmarking of Radiation-Hydrodynamic Modeling, Lawrence Livermore National Laboratory, Livermore, CA, UCRL-JC133739. Submitted to J. Quant. Spectros. and Radiat. Transfer.

Glenzer, S. H., Geddes, C. G., Kirkwood, R. K., MacGowan, B. J., Moody, J. D., and Young, P. E., Thomson Scattering on Ion Waves Driven by SBS in Large-Scale Length Plasmas, Lawrence Livermore National Laboratory, Livermore, CA, UCRL-JC133945-ABS. Prepared for 29th Annual Anomalous Absorption Conf, Pacific Grove, CA, Jun 13, 1999.
Glenzer, S. H., MacGowan, B. J., Young, P. E., and De Groot, J. S., Thomson Scattering for Basic Science Experiments, Lawrence Livermore National Laboratory, Livermore, CA, UCRL-JC-135311 ABS. Prepared for Frontier Science at the National Ignition Facility (NIF) Workshop, Pleasanton, CA, Oct 4, 1999.

Glenzer, S. H., Rozmus, W., MacGowan, B. J., Estabrook, K. G., De Groot, J. D., Zimmerman, G. B., Baldis, H. A., Hammel, B. A., Harte, J. A., Lee, R. W., Williams, E. A., and Wilson, B. G., Thomson Scattering. from High-Z Laser-Produced Plasmas, Lawrence Livermore National Laboratory, Livermore, CA, UCRL-JC-131980; also in Phys. Rev. Lett. 82(1), 97-100 (1999).

Glenzer, S. H., Suter, L. J., Estabrook, K. G., and Baldis, H. A., Thomson Scattering from Inertial Confinement Fusion Targets, Lawrence Livermore National Laboratory, Livermore, CA, UCRL-JC-134883.

Prepared for 9th Intl Symp on Laser-Aided Plasma Diagnostics, Lake Tahoe, CA, Sep 26, 1999.

Glenzer, S. H., Thomson Scattering in Inertial Confinement Fusion Research, Lawrence Livermore National Laboratory, Livermore, CA, UCRL-JC-135822. Submitted to Contributions to Plasma Physics.

Gold, D. M., Celliers, P. M., Collins, G. W., Da Silva, L. B., Cauble, R. C., Kalantar, D. H., Weber, S. V., and Remington, B. A., Optical Interferometry Diagnostics in LaserDriven Equation of State Experiments, Lawrence Livermore National Laboratory, Livermore, CA, UCRL-JC-134798. Prepared for 11th Topical Conf on Shock Compression in Condensed Matter, Snowbird, UT, Jun 27, 1999.

Gresho, P. M., Some Aspects of the Hydrodynamics of the Microencapsulation Route to NIF Mandrels, Lawrence Livermore National Laboratory, Livermore, CA, UCRL-JC-129963; also in Fusion Tech. 35(2), 157-188 (1999). 


\section{$\mathbf{H}$}

Haan, S. W., Jones, O. S., Dittrich, T. R., Koch, J. A., and Sangster, T. C., Diagnosis of Ignition Shots on NIF, Lawrence Livermore National Laboratory, Livermore, CA, UCRL-JC-133724 ABS Rev 1. Prepared for Nuclear Explosives Design Physics Conf, Los Alamos, NM, Oct 25, 1999.

Haan, S. W., Jones, O. S., Koch, J. A., Sangster, T. C., Pollaine, S. M., Dittrich, T. R., Hinkel, D. E., and Marinak, M. M., Target Simulations for Diagnostic Design for Ignition Targets for the National Ignition Facility, Lawrence Livermore National Laboratory, Livermore, CA, UCRL-JC131431 ABS Rev. Prepared for 41st Annual Mtg of the American Physical Society Div of Plasma Physics, Seattle, WA, Nov 15, 1999.

Haber, I., Friedman, A., Grote, D. P., Lund, S. M., and Kishek, R. A., "Recent Progress in the Simulation of Heavy Ion Beams," Phys. Plasmas 6(5), Pt. 2, 2254-2261 (1999).

Hammel, B. A., Bernat, T. P., Collins, G. W., Haan, S., Landen, O. L., MacGowan, B. J., and Suter, L. J., Recent Advances in Indirect Drive ICF Target Physics at LLNL, Lawrence Livermore National Laboratory, Livermore, CA, UCRL-JC-130302.

Prepared for 17th Intl Atomic Energy Agency Fusion Energy Conf, Yokohama, Japan, Oct 19, 1998.

Hammer, J. H., and Ryutov, D. D., Linear Stability of an Accelerated Wire Array, Lawrence Livermore National Laboratory, Livermore, CA, UCRL-JC-132255; also in Phys. Plasmas 6(8), 3302-3315 (1999).

Hammer, J. H., Tabak, M., Wilks, S. C., Bailey, D. S., Koch, J., Landen, O., Lindl, J., Rambo, P. W., Toor, A., and Zimmerman, G. B., High Yield ICF with a Z-Pinch Driven Hohlraum, Lawrence Livermore National Laboratory, Livermore, CA, UCRL-JC133657-ABS. Prepared for 1st Intl Conf on Inertial Fusion Sciences and Applications (IFSA), Bordeaux, France, Sept 12, 1999.
Hammer, J. H., Tabak, M., Wilks, S. C., Lindl, J. D., Bailey, D. S., Rambo, P. W., Toor, A., Zimmerman, G. B., and Porter, J. L., High Yield ICF Target Design for a Z-Pinch Driven Hohlraum, Lawrence Livermore National Laboratory, Livermore, CA, UCRL-JC-131389. Prepared for 40th Annual Mtg of the Div of Plasma Physics, New Orleans, LA, Nov 16, 1998; also in Phys. Plasmas 6(5), Pt. 2, 2129-2136 (1999).

Hammer, J. H., Tabak, M., Wilks, S., Lindl, J., Rambo, P. W., Toor, A., Zimmerman, G. B., and Porter, J. L., High Yield Inertial Fusion Design for a Z-Pinch Accelerator, Lawrence Livermore National Laboratory, Livermore, CA, UCRL-JC-132152. Prepared for 17th Intl Atomic Energy Agency Fusion Energy Conf, Yokohama, Japan, Oct 19, 1998.

Hassel, A. E., Roberts, C. C., and Letts, S. A., Molecular Weight Studies of Polyimide Materials for NIF Capsules, Lawrence Livermore National Laboratory, Livermore, CA, UCRL-JC-135499 ABS. Prepared for Target Fabrication Mtg '99, Catalina Island, CA, Nov 8, 1999.

Hatchett, S., Snavely, R. A., Key, M., Brown, C., Cowan, T., Henry, G., Langdon, B., Lasinski, B., Pennington, D., and Perry, M., Observation and Interpretation of the Angular Patterns and Characteristic Energies of Relativistic Electrons Ejected from Petawatt Laser Targets, Lawrence Livermore National Laboratory, Livermore, CA, UCRL-JC-133725-ABS. Prepared for Anomalous Absorption Conf, Monterey, CA, Jun 13, 1999.

Herrmann, M. C., Tabak, M., and Lindl, J., Generalized Scaling Law for the Ignition Energy of ICF Capsules, Lawrence Livermore National Laboratory, Livermore, CA, UCRL-JC-134888 ABS. Prepared for 41st Annual Mtg of the American Physical Society Div of Plasma Physics, Seattle, WA, Nov 15, 1999. 
Hinkel, D. E., and Haan, S. W., Laser-Plasma Interactions in the 350-eV National Ignition Facility Target, Lawrence Livermore National Laboratory, Livermore, CA, UCRL-JC-133083-ABS Rev. Prepared for 29th Annual Anomalous Absorption Conf, Pacific Grove, CA, Jun 13, 1999.

Hinkel, D. E., Berger, R. L., Williams, E. A., Langdon, A. B., Still, C. H., and Lasinski, B. F., Stimulated Brillouin Backscatter in the Presence of Transwerse Plasma Flow, Lawrence Livermore National Laboratory, Livermore, CA, UCRL-JC-130345; also in Phys. Plasmas 6(2), 571-581 (1999).

Hinkel, D. E., Haan, S. W., and Dittrich, T. R., National Ignition Facility Targets Driven at High Radiation Temperatures, Lawrence Livermore National Laboratory, Livermore, CA, UCRL-JC-135013 ABS. Prepared for 41st Annual Mtg of the American Physical Society Div of Plasma Physics, Seattle, WA, Nov 15, 1999.

Hinkel, D. E., Haan, S. W., Dittrich, T. R., and Marinak, M. M., Design of Ignition Targets for the National Ignition Facility, Lawrence Livermore National Laboratory, Livermore, CA, UCRL-JC-133510-ABS. Prepared for 1st Intl Conf on Inertial Fusion Sciences and Applications, Bordeaux, France, Sept 12, 1999.

Ho, D. D. M., Grabowski, J. J., Brandon, S. T., Tsang, K. T., and Wong, A. Y., Transfer of Free Electrons to Chlorine and Its Consequences for Mitigating Ozone Depletion in a Computational Model of the Antarctic Stratosphere, Lawrence Livermore National Laboratory, Livermore, CA, UCRL-JC120003 Rev 1; also in Phys. Essays 11(4), 569-575 (1998).

Ho, D. D.-M., Chen, Y.-J., Pincosy, P. A., Young, D. A., and Bergstrom, P. M., Hydrodynamic Modeling of the Plasma Plume Expansion from the Targets for X-Ray Radiography, Lawrence Livermore National Laboratory, Livermore, CA, UCRL-JC132181-ABS. Prepared for 1999 Particle Accelerator Conf, New York City, NY, Mar $29,1999$.
Hurricane, O. A., Remington, B. A., Drake, R. P., Robey, H., and Glendinning, S. G., CALE Simulation of the Spherically Divergent NLUF 2 Experiment, Lawrence Livermore National Laboratory, Livermore, CA, UCRL-JC-134963 ABS. Prepared for 41st Annual Mtg of the American Physical Society Div of Plasma Physics, Seattle, WA, Nov 15, 1999.

\section{I}

Isakov, A. I., Annual Report 1998, Lawrence Livermore National Laboratory, Livermore, CA, UCRL-CR-133636 (1998).

J

Johnson, R. R., Emergence of the National Ignition Facility, Lawrence Livermore National Laboratory, Livermore, CA, UCRL-JC-133034-ABS. Prepared for Optical Society of America Mtg Section Mtg, Ann Arbor, MI, Feb 23, 1999.

Jones, O. S., Suter, L. J., Marinak, M. M., and Kerbel, G. D., Design of Plasma Emulators for NIF, Lawrence Livermore National Laboratory, Livermore, CA, UCRL-JC-135025 ABS. Prepared for 41st Annual Mtg of the American Physical Society Div of Plasma Physics, Seattle, WA, Nov 15, 1999.

Jones, O. S., Suter, L. J., Marinak, M. M., and Kerbel, G. D., Radiation Environment in Nonsymmetric NIF Hohlraums, Lawrence Livermore National Laboratory, Livermore, CA, UCRL-JC-134038-ABS. Prepared for 29th Annual Anomalous Absorption Conf, Pacific Grove, CA, Jun 13, 1999.

Jones, R., Spragge, M., Burmann, J., and Rivers, C., Water Equation of State Targets, Lawrence Livermore National Laboratory, Livermore, CA, UCRL-JC-135538 ABS. Prepared for Target Fabrication Mtg '99, Catalina Island, CA, Nov 8, 1999. 
K

Kalantar, D. H., and Hsing, W., Evaluation of Illumination Geometry for the UK Chamber on NIF, Lawrence Livermore National Laboratory, Livermore, CA, UCRL-JC135366 ABS. Prepared for Joint Operations Working Group 37, Aldermaston, UK, Sep 20, 1999 .

Kalantar, D. H., Chandler, E. A., Colvin, J. D., Lee, R., Remington, B. A., Weber, S. V., Wiley, L. G., Hauer, A., Wark, J. S., Loveridge, A., Failor, B. H., Meyers, M. A., and Ravichandran, G., Transient X-Ray Diffraction Used to Diagnose Shock Compressed Si Crystals on the Nova Laser, Lawrence Livermore National Laboratory, Livermore, CA, UCRL-JC-129861; also in Rev. Sci. Inst. 70(1), 629-632 (1999).

Kalantar, D. H., Colvin, J., Gold, D., Mikaelian, K., Remington, B. A., Weber, S. V., Wiley, L., Allen, A., Loveridge, A., and Wark, J., High-Pressure Solid-State Experiments on Intense Lasers, Lawrence Livermore National Laboratory, Livermore, CA, UCRL-JC-134965 ABS. Prepared for 1999 Nuclear Explosives Design Physics Conf, Los Alamos, NM, Oct 25, 1999.

Kalantar, D. H., Colvin, J., Remington, B., Weber, S., Wiley, L., Wark, J. S., Loveridge, A., Allen, A., Hauer, A. A., and Paisley, D., Transient X-Ray Diffraction from Shock Compressed Si and $\mathrm{Cu}$ Crystals, Lawrence Livermore National Laboratory, Livermore, CA, UCRL-JC-135561 ABS. Prepared for Frontier Science at the NIF: Episode I, Pleasanton, CA, Oct 4, 1999.

Kalantar, D. H., Experiment Design for the National Ignition Facility, Lawrence Livermore National Laboratory, Livermore, CA, UCRL-JC-135474 ABS. Prepared for Frontier Science at the NIF: Episode I, Pleasanton, CA, Oct 4, 1999.

Kalantar, D. H., Impact of Unconverted Light on Diagnostics for NIF Experiments, Lawrence Livermore National Laboratory, Livermore, CA, UCRL-JC-133225-ABS. Prepared for the Workshop on Target Diagnostics for Large Laser Fusion Facilities, Monterey, CA, Mar 1, 1999.
Kalantar, D. H., Probing Shock Compressed Solids by Dynamic X-Ray Diffraction, Lawrence Livermore National Laboratory, Livermore, CA, UCRL-JC-134964 ABS. Prepared for 41st Annual Mtg of the American Physical Society Div of Plasma Physics, Seattle, WA, Nov 15, 1999.

Kalantar, D. H., Remington, B. A., Chandler, E. A., Colvin, J. D., Gold, D. M., Mikaelian, K. O., Weber, S. V., Wiley, L. G., Wark, J. S., and Hauer, A. A., High Pressure Solid State Experiments on the Nova Laser, Lawrence Livermore National Laboratory, Livermore, CA, UCRL-JC-129810.

Prepared for 1998 Hypervelocity Impact Symp, Huntsville, AL, Nov 16, 1998.

Kalantar, D. H., Remington, B. A., Chandler, E. A., Colvin, J. D., Gold, D. M., Mikaelian, K. O., Weber, S. V., Wiley, L. G., Wark, J. S., and Loveridge, A., Developing Solid State Experiments on the Nova Laser, Lawrence Livermore National Laboratory, Livermore, CA, UCRL-JC-131851. Prepared for 2 nd Intl Workshop on Laboratory Astrophysics with Intense Lasers, Tucson, AZ, Mar 19, 1998.

Kalantar, D. H., Remington, B. A., Colvin, J. D., Gold, D. M., Mikaelian, K. O., Weber, S. V., and Wiley, L. G., Shock Compressed Solids on the Nova Laser, Lawrence Livermore National Laboratory, Livermore, CA, UCRL-JC-132687 ABS. Prepared for 11th Topical Conf on Shock Compression of Condensed Matter, Snowbird, UT, Jun 27, 1999.

Kalantar, D. H., Remington, B. A., Colvin, J. D., Gold, D. M., Mikaelian, K. O., Weber, S. V., Wiley, L. G., Allen, A. M., Loveridge, A., and Wark, J. S., Laser Driven Shock Compressed Solid State RT and Diffraction Experiments, Lawrence Livermore National Laboratory, Livermore, CA, UCRL-JC135436 ABS. Prepared for Joint Operations Weapons Operations Group 37, Aldermaston, UK, Sep 20, 1999.

Kalantar, D., and Dixit, S., Baseline Unconverted Light Management Plan (Indirect Drive Configuration), Lawrence Livermore National Laboratory, Livermore, CA, UCRL-ID-134173. 
Kalantar, D., DIM and Diagnostic Placement for NIF Experiments, Lawrence Livermore National Laboratory, Livermore, CA, UCRL-ID-135801.

Kalantar, D., Dixit, S., and Lyons, R., Impact of Zero Order Unconverted Light on Beam Pointing, Lawrence Livermore National Laboratory, Livermore, CA, UCRL-ID-135816.

Kane, J., Arnett, D., Remington, B. A., Glendinning, S. G., Bazan, G., Muller, E., Fryxell, B. A., and Teyssier, R., $2 d$ vs. $3 d$ Supernova Hydrodynamic Instability Growth, Lawrence Livermore National Laboratory, Livermore, CA, UCRL-JC-133050.

Submitted to Astrophys. J.

Kane, J., Arnett, D., Remington, B. A., Glendinning, S. G., Bazan, S. G., Drake, R. P., Fryxell, B. A., Teyssier, R., and Moore, K., Scaling Supernova Hydrodynamics to the Laboratory, Lawrence Livermore National Laboratory, Livermore, CA, UCRL-JC132438; also in Phys. Plasmas 6(5), Pt.2, 2065-2071 (1999).

Kane, J., Drake, R. P., and Remington, B. A., An Evaluation of the Richtmyer-Meshkov Instability in Supernova Remnant Formation, Lawrence Livermore National Laboratory, Livermore, CA, UCRL-JC-130957; also in Astrophys. J. 511(1,PT1), 335-340 (1999).

Kartz, M., Olivier, S., Brase, J., Carrano, C., Silva, D., Pennington, D., and Brown, C., High Resolution Wavefront Control of HighPower Laser Systems, Lawrence Livermore National Laboratory, Livermore, CA, UCRL-JC-134822. Prepared for Intl Workshop on Adaptive Optics for Industry and Medicine, Durham, England, Jul 12, 1999.

Kastenberg, W. E., Industrial Ecology Analysis Final Report, Lawrence Livermore National Laboratory, Livermore, CA, UCRL-CR-132271.

Kauffman, R., 1998 Inertial Confinement Fusion Annual Report, Lawrence Livermore National Laboratory, Livermore, CA, UCRL-LR-105820-98.
Kauffman, R., ICF Quarterly Report, 8(4), Lawrence Livermore National Laboratory, Livermore, CA, UCRL-LR-105821-98-4 (1998).

Kauffman, R., Inertial Confinement Fusion Monthly Highlights, September 1998, Lawrence Livermore National Laboratory, Livermore, CA, UCRL-TB-128550-98-12.

Kauffman, R., Inertial Confinement Fusion Monthly Highlights, October 1998, Lawrence Livermore National Laboratory, Livermore, CA, UCRL-TB-128550-99-1.

Kauffman, R., Inertial Confinement Fusion Monthly Highlights, November 1998,

Lawrence Livermore National Laboratory, Livermore, CA, UCRL-TB-128550-99-2.

Kauffman, R., Inertial Confinement Fusion Monthly Highlights, December 1998, Lawrence Livermore National Laboratory, Livermore, CA, UCRL-TB-128550-99-3.

Kauffman, R., Inertial Confinement Fusion Monthly Highlights, January 1999,

Lawrence Livermore National Laboratory, Livermore, CA, UCRL-TB-128550-99-4.

Kauffman, R., Inertial Confinement Fusion Monthly Highlights, February 1999,

Lawrence Livermore National Laboratory, Livermore, CA, UCRL-TB-128550-99-5.

Kauffman, R., Inertial Confinement Fusion Monthly Highlights, March 1999, Lawrence Livermore National Laboratory, Livermore, CA, UCRL-TB-128550-99-6.

Kauffman, R., Inertial Confinement Fusion Monthly Highlights, April 1999, Lawrence Livermore National Laboratory, Livermore, CA, UCRL-TB-128550-99-7.

Kauffman, R., Inertial Confinement Fusion Monthly Highlights, May 1999, Lawrence Livermore National Laboratory, Livermore, CA, UCRL-TB-128550-99-8.

Kauffman, R., Inertial Confinement Fusion Monthly Highlights, June 1999, Lawrence Livermore National Laboratory, Livermore, CA, UCRL-TB-128550-99-9.

Kauffman, R., Inertial Confinement Fusion Monthly Highlights, July 1999, Lawrence Livermore National Laboratory, Livermore, CA, UCRL-TB-128550-99-10. 
Kauffman, R., Inertial Confinement Fusion Monthly Highlights, August 1999,

Lawrence Livermore National Laboratory, Livermore, CA, UCRL-TB-128550-99-11.

Keilty, K. A., Liang, E. P., Ditmire, T. R., Remington, B. A., Rubenchik, A. M., and Shigemori, K., Modeling of Laser-Generated Radiative Blast Waves, Lawrence Livermore National Laboratory, Livermore, CA, UCRL-JC-135573 ABS. Prepared for Frontier Science at the NIF: Episode I, Pleasanton, CA, Oct 4, 1999.

Key, M. H., Campbell, E. M., Cowan, T. E., Hammel, B. A., Hatchett, S. P., Henry, E. A., Kilkenny, J. D., Koch, J. A., Langdon, A. B., and Lasinski, B. F., Studies of the Prospects for Fast Ignition Using a Petawatt Laser Facility, Lawrence Livermore National Laboratory, Livermore, CA, UCRL-JC-134181-ABS. Prepared for Advanced High Power Lasers and Applications, Osaka, Japan, Nov 1, 1999.

Key, M. H., Cowan, T. E., Hammel, B. A., Hatchett, S. P., Henry, E. A., Kilkenny, J. D., Koch, J. A., Langdon, A. B., Lasinski, B. F., and Lee, R. W., Progress in Fast Ignitor Research with the Nova Petawatt Laser Facility, Lawrence Livermore National Laboratory, Livermore, CA, UCRL-JC132178. Prepared for 17th Intl Atomic Energy Agency Fusion Energy Conf, Yokohama, Japan, Oct 19, 1998.

Key, M. H., Laser Interactions with Matter at Extremely High Intensities, Lawrence Livermore National Laboratory, Livermore, CA, UCRL-JC-133049 ABS. Prepared for Italian National Institute of Matter Physics, Sicily, Italy, Jun 14, 1999.

Key, M., ICF Quarterly Report, 8(3), Lawrence Livermore National Laboratory, Livermore, CA, UCRL-LR-105821-98-3 (1998).

Kilkenny, J. D., Bernat, T. P., Hammel, B. A., Kauffman, R. L., Landen, O. L., Lindl, J. D., MacGowan, B. J., Paisner, J. A., and Powell, H. T., Lawrence Livermore National Laboratory's Activities to Achieve Ignition by $X$-Ray Drive on the National Ignition Facility, Lawrence Livermore National Laboratory, Livermore, CA, UCRL-JC-131510; also in Laser and Part. Beams 17(2), 159-171 (1999).
Kirkwood, R. K., Berger, R. L., Geddes, C. G., Glenzer, S. H., Langdon, A. B., MacGowan, B. J., Moody, J. D., and Young, P. E., Experimental Study of the Effect of Speckle Size on Backscatter in a Phase Plate Smoothed Beam, Lawrence Livermore National Laboratory, Livermore, CA, UCRL-JC-133944-ABS. Prepared for 29th Annual Anomalous Absorption Conf, Pacific Grove, CA, Jun 13, 1999.

Kirkwood, R. K., Joshi, C., Glenzer, S. H., Geddes, C. G. R., Afeyan, B. B., Wharton, K. B., Estabrook, K. G., Cohen, B. I., and Moody, J. D., Advanced Studies of NonLinear, Multi-Wave, Processes Using the National Ignition Facility, Lawrence Livermore National Laboratory, Livermore, CA, UCRL-JC-135422 ABS Prepared for Frontier Science at the NIF: Episode I, Pleasanton, CA, Oct 4, 1999.

Kirkwood, R. K., Suter, L. J., Glenzer, S. H., Geddes, C. G. R., Miller, M., and Hsing, W. W., Bremsstrahlung X-Ray Source Driven by Stimulated Raman Scattering of $351 \mathrm{~nm}$ Light, Lawrence Livermore National Laboratory, Livermore, CA, UCRL-JC-134958 ABS.

Prepared for 41st Annual Mtg of the American Physical Society Div of Plasma Physics, Seattle, WA, Nov 15, 1999.

Koch, J. A., Aglitskiy, Y., and Brown, C., Bragg Crystals for High-Energy, HighResolution X-Ray Imaging Applications at the National Ignition Facility, Lawrence Livermore National Laboratory, Livermore, CA, UCRL-JC-135509 ABS. Prepared for 2nd Workshop on Methods $\mathcal{E}$ Applications of Curved Crystal X-ray Optics, Weimar, Germany, Oct 4, 1999.

Koch, J. A., Bittner, D., Collins, G., Geddes, C., Lee, Y., MacKinnon, A., and Sater, J., Quantitative Analysis of Shadowgraphy and Interferometry as Diagnostics of Hydrogen Ice Surface Quality in ICF Capsules, Lawrence Livermore National Laboratory, Livermore, CA, UCRL-JC-135496 ABS. Prepared for Target Fabrication Mtg '99, Catalina Island, CA, Nov 8, 1999. 
Koch, J. A., Cui, S., and McNeill, M., Effective Focal Length Calculations and Measturements for a Radial Diffraction Grating, Lawrence Livermore National Laboratory, Livermore, CA, UCRL-JC133594. Submitted to J. Opt. Society of America A.

Koch, J. A., Hatchett, S. P., Key, M. H., Lee, R. W., Pennington, D., Stephens, R. B., and Tabak, M., Observation of Deep Directional Heating at Near-Solid Density Caused by Laser-Generated Relativistic Electrons, Lawrence Livermore National Laboratory, Livermore, CA, UCRL-JC-135139. Submitted to Phys. Rev. Lett.

Koch, J. A., Landen, O. L., Hammel, B. A., Brown, C., Seely, J., and Aglitskiy, Y., Recent Progress in High-Energy, HighResolution X-Ray Imaging Techniques for Application to the National Ignition Facility, Lawrence Livermore National Laboratory, Livermore, CA, UCRL-JC-129869; also in Rev. Sci. Inst. 70(1), 525-5629 (1999).

Koch, J., Hatchett, S. P., Key, M. H., Lee, R. W., Pennington, D., Stephens, R. B., and Tabak, M., Measurements of Deep Heating Generated by Ultra-Intense Laser-Plasma Interactions, Lawrence Livermore National Laboratory, Livermore, CA, UCRL-JC133686. Prepared for 1st Intl Conf on Inertial Fusion Sciences and Applications, Bordeaux, France, Sep 12, 1999.

Koch, J., Hatchett, S., Key, M., Lee, R., Pennington, D., Stephens, R., and Tabak, M., Experimental Measurements of Deep Directional Heating at Near-Solid Density Caused by Laser-Generated Relativistic Electrons, Lawrence Livermore National Laboratory, Livermore, CA, UCRL-JC135064 ABS. Prepared for 41st Annual Mtg of the American Physical Society Div of Plasma Physics, Seattle, WA, Nov 15, 1999.

Koch, J., Key, M., Lee, R., Hatchett, S., Brown, C., and Pennington, D., Experimental Measurements of Deep Heating Generated by Ultra-Intense Laser-Plasma Interactions, Lawrence Livermore National Laboratory, Livermore, CA, UCRL-JC133831-ABS. Prepared for 29th Annual Anomalous Absorption Conf, Pacific Grove, CA, Jun 13, 1999.
Kodama, R., Takahashi, K., Tanaka, K. A., Kato, Y., Murai, K., Weber, F., Barbee, T. W., and Da Silva, L. B., "Measurements of Laser-Hole Boring into Overdense Plasmas Using X-Ray Laser Refractometry," Rev. Sci. Inst. 70(1), 543-548 (1999).

Kozioziemski, B. J., Collins, G. W., Bernat, T. P., and Sater, J. D., 3He Evolution in Deuterium-Tritium Layers, Lawrence Livermore National Laboratory, Livermore, CA, UCRL-JC-135484 ABS. Prepared for Target Fabrication Mtg '99, Catalina Island, CA, Nov 8, 1999.

Kozioziemski, B., Collins, G. W., Bernat, T. P., and Sater, J. D., Helium Bubble Dynamics in D-T Layers, Lawrence Livermore National Laboratory, Livermore, CA, UCRL-JC-130038 ABS Rev. Prepared for 1999 Centennial Mtg, Atlanta, GA, Mar 20, 1999.

Kozlowski, M. R., Demos, S. G., and Battersby, C. L., Spectroscopic Investigation of $\mathrm{SiO}_{2}$ Surfaces Damaged by 351-nm Laser Illumination, Lawrence Livermore National Laboratory, Livermore, CA, UCRL-JC134818 ABS. Prepared for 31st Boulder Damage Symp: Annual Symp on Optical Materials for High Power Lasers, Boulder, CO, Oct 4, 1999.

Kruer, W. L., Decker, C. D., Estabrook, K. G., Rambo, P. W., and Wilks, S. C., Controlling Laser Plasma Interactions in Ignition-Scale Hohlraums, Lawrence Livermore National Laboratory, Livermore, CA, UCRL-JC-135020 ABS. Prepared for 41st Annual Mtg of the American Physical Society Div of Plasma Physics, Seattle, WA, Nov 15, 1999.

Kruer, W., Inertial Confinement Fusion Quarterly Report, January-March 1998, Lawrence Livermore National Laboratory, Livermore, CA, UCRL-LR-105821-98-2. 
L

Labaune, C., Baldis, H. A., Cohen, B., Rozmus, W., Depierreux, S., Schifano, E., Bauer, B. S., and Michard, A., Nonlinear Modification of Laser-Plasma Interaction Processes under Crossed Laser Beams, Lawrence Livermore National Laboratory, Livermore, CA, UCRL-JC-132581; also in Phys. Plasmas 6(5), Pt. 2, 2048-2056 (1999).

Landen, N., NIF Diagnostic Damage and Design Issues, Lawrence Livermore National Laboratory, Livermore, CA, UCRL-ID-134644.

Landen, O. L., Amendt, P. A., Suter, L. J., Turner, R. E., Glendinning, S. G., Haan, S. W., Pollaine, S. M., Hammel, B. A., Tabak, M., Rosen, M. D., and Lindl, J. D., A Simple Time-Dependent Analytic Model of the $P_{2}$ Asymmetry in Cylindrical Hohlraums, Lawrence Livermore National Laboratory, Livermore, CA, UCRL-JC-132445; also in Phys. Plasmas 6(5), Pt. 2, 2137-2143 (1999).

Landen, O. L., Amendt, P. A., Turner, R. E., Bradley, D. K., Suter, L. J., Wallace, R. J., and Hammel, B. A., High Convergence Implosion Symmetry in Cylindrical Hohlraums, Lawrence Livermore National Laboratory, Livermore, CA, UCRL-JC135811. Prepared for 1st Intl Conf on Inertial Fusion Sciences and Applications, Bordeaux, France, Sep 12, 1999.

Landen, O. L., Amendt, P. A., Turner, R. E., Glendinning, S. G., Kalantar, D. H., Decker, C. D., Suter, L. J., Cable, M. D., Wallace, R., and Hammel, B. A., TimeDependent, Indirect-Drive Symmetry Control on the Nova and Omega Facilities, Lawrence Livermore National Laboratory, Livermore, CA, UCRL-JC-130109. Prepared for 17th Intl Atomic Energy Agency Fusion Energy Conf, Yokohama, Japan, Oct 19, 1988.

Landen, O. L., Back, C. A., Bauer, J., Hammer, J., Koch, J., Rambo, P., Lasinski, B., Rosen, M., and Hising, W. W., Radiation Transport on Nova/OMEGA to Z to NIF, Lawrence Livermore National Laboratory, Livermore, CA, UCRL-JC-135439 ABS. Prepared for Joint Operations Weapons Operations Group 37, Aldermaston, UK, Sep 20, 1999.
Landen, O. L., Bradley, D. K., Pollaine, S. M., Amendt, P. A., Glendinning, S. G., Suter, L. J., Turner, R. E., Wallace, R. J., Hammel, B. A., and Delamater, N., Indirect-Drive Symmetry Diagnosis at NIFScale Using the OMEGA Laser Facility, Lawrence Livermore National Laboratory, Livermore, CA, UCRL-JC-133632-ABS. Prepared for 1st Intl Conf on Inertial Fusion Sciences and Applications, Bordeaux, France, Sept 12, 1999.

Landen, O. L., Dense Plasma Characterization by Soft X-Ray Laser Thomson Scattering on the NIF, Lawrence Livermore National Laboratory, Livermore, CA, UCRL-JC-135475 ABS Rev. Prepared for Frontier Science at the NIF: Episode I, Pleasanton, CA, Oct 4, 1999.

Landen, O. L., Farley, D. R., Glendinning, S. G., Logory, L. M., Bell, P. M., Koch, J. A., Lee, F. D., Bradley, D. K., Kalantar, D. H., and Back, C. A., Backlighting Developments for HED on NIF, Lawrence Livermore National Laboratory, Livermore, CA, UCRL-JC-135434 ABS. Prepared for Joint Operations Weapons Operations Group 37, Aldermaston, UK, Sep 20, 1999.

Lane, M. A., Van Wonterghem, B. M., and Clower Jr., C. A., Phased NIF Start-Up, Lawrence Livermore National Laboratory, Livermore, CA, UCRL-JC-129271; also in Fusion Tech. 34(3), Pt. 2, 1127-1134 (1998).

Langer, S. H., Scott, H. A., Marinak, M. M., and Landen, O. L., Modeling Line Emission from ICF Capsules in 3 Dimensions,

Lawrence Livermore National Laboratory, Livermore, CA, UCRL-JC-131240.

Submitted to J. Quant. Spectros. Radiat. Transfer.

Lasinski, B. F., Langdon, A. B., Hatchett, S. P., Key, M. H., and Tabak, M., Particle in Cell Simulations of Ultra Intense Laser Pulses Propagating Through Overdense Plasma for Fast-Ignitor and Radiography Applications, Lawrence Livermore National Laboratory, Livermore, CA, UCRL-JC-131245; also in Phys. Plasmas 6(5), Pt. 2, 2041-2047 (1999). 
Latkowski, J. F., Neutron Activation of the NIF Final Optics Assemblies and Their Effect upon Occupational Doses, Lawrence Livermore National Laboratory, Livermore, CA, UCRL-JC-129515; also in Fusion Tech. 34(3), Pt. 2, 767-771 (1998).

Latkowski, J. F., Sanchez, J. J., and Pittenger, L. C., Neutronics and Activation Analysis for the National Ignition Facility Cryogenic Target Positioner, Lawrence Livermore National Laboratory, Livermore, CA, UCRL-JC-129954; also in Fusion Tech. 35(2), 255-259 (1999).

Leeper, R. J., Alberts, T. E., Asay, J. R., Baca, P. M., Baker, K. L., Breeze, S. P., Chandler, G. A., Cook, D. L., Cooper, G. W., Deeney, C., Derzon, M. S., Douglas, M. R., Fehl, D. L., Gilliland, T., Hebron, D. E., Hurst, M. J., Jobe, D. O., Kellogg, J. W., Lash, J. S., Lazier, S. E., Matzen, M. K., McDaniel, D. H., McGurn, J. S., Mehlhorn, T. A., Moats, A. R., Mock, R. C., Muron, D. J., Nash, T. J., Olson, R. E., Porter, J. L., Quintenz, J. P., Reyes, P. V., Ruggles, L. S., Ruiz, C. L., Sanford, T. W. L., Schmidlapp, F. A., Seamen, J. F., Spielman, R. B., Stark, M. A., Struve, K. W., Stygar, W. A., Tibbetts-Russell, D. R., Torres, J. A., Vargas, T., Wagoner, T. C., Wakefield, C., Hammer, J. H., Ryutov, D. D., Tabak, M., Wilks, S. C., Bowers, R. L., McLenithan, K. D., and Peterson, D. L., "Z Pinch Driven Inertial Confinement Fusion Target Physics Research at Sandia National Laboratories," Nuclear Fusion 39(9Y 1), 1283-1294 (1999).

Lerche, R. A., and Ognibene, T. J., Error Analysis for Fast Scintillator-Based Inertial Confinement Fusion Burn History Measurements, Lawrence Livermore National Laboratory, Livermore, CA, UCRL-JC-129701; also in Rev. Sci. Inst. 70(1), 1217-1219 (1999).

Letts, S. A., Buckley, S., Roberts, C. C., Orthion, P., Fearon, E., and Cook, R. C., Development of Upilex Coatings for Laser Targets, Lawrence Livermore National Laboratory, Livermore, CA, UCRL-JC135486 ABS. Prepared for Target Fabrication Mtg '99, Catalina Island, CA, Nov 8, 1999.
Letts, S. A., Parrish, B., Rogers, G., Buckley, S., and Saculla, M., Thermal Properties of Plasma Polymers, Lawrence Livermore National Laboratory, Livermore, CA, UCRL-JC-135593 ABS. Prepared for Target Fabrication Mtg '99, Catalina Island, CA, Nov 8, 1999.

Liang, E. P., Wilks, S. C., and Tabak, M., Pair Production by Ultraintense Lasers, Lawrence Livermore National Laboratory, Livermore, CA, UCRL-JC-124499 Rev 1; also in Phys. Rev. Lett. 81(22), 4887-4890 (1998).

Lindl, J., Herrmann, M., and Tabak, M., Generalized Scaling of the Energy Threshold for Ignition and Efficient Burn of ICF Targets, Lawrence Livermore National Laboratory, Livermore, CA, UCRL-JC-133642-ABS. Prepared for 1st Intl Conf on Inertial Fusion Sciences and Applications, Bordeaux, France, Sept 12, 1999.

Logan, B. G., Ditmire, T. R., Perry, M. D., and Caporaso, G. J., Model for a ShortPulse-Laser-Driven, High-Peak-Current Ion Source, Lawrence Livermore National Laboratory, Livermore, CA, UCRL-JC135098 ABS. Prepared for 1st Intl Conf on Inertial Fusion Sciences and Applications, Bordeaux, France, Sep 12, 1999.

Lowenthal, M. D., Greenspan, E., Moir, R., Kastenberg, W. E., and Fowler, T. K., "Industrial Ecology for Inertial Fusion Energy: Selection of High-Z Material for HYLIFE-II Targets," Fusion Tech. 34(3), Pt. 2, 619-628 (1998).

\section{M}

MacGowan, B. J., Berger, R. L., Cohen, B. I., Decker, C. D., Dixit, S., Glenzer, S. H., Hinkel, D. E., Kirkwood, R. K., Langdon, A. B., and Lefebvre, E., Laser Beam Smoothing and Backscatter Saturation Processes in Plasmas Relevant to National Ignition Facility Hohlraums, Lawrence Livermore National Laboratory, Livermore, CA, UCRL-JC-132191. Prepared for 17th Intl Atomic Energy Agency Fusion Energy Conf, Yokohama, Japan, Oct 19, 1988. 
MacKinnon, A. J., Brown, C. G., Ditmire, T., Hatchett, S., Koch, J., Key, M. H., Moody, J., Offenberger, A. A., Pennington, D., and Perry, M. D., Study of the Interaction of Ultra-Intense Laser Pulses with Solid Targets on the Petawatt Laser System, Lawrence Livermore National Laboratory, Livermore, CA, UCRL-JC-133976-ABS.

Prepared for 29th Annual Anomalous Absorption Conf, Pacific Grove, CA, Jun 13, 1999.

MacKinnon, A. J., Brown, C. G., Ditmire, T., Hatchett, S., Koch, J., Key, M. H., Moody, J., Pennington, D., Perry, M. D., and Wilks, S. C., Study of the Interaction of Ultra-Intense Laser Pulses with Solid Targets and Preformed Plasmas with Applications to the Fast Ignitor, Lawrence Livermore National Laboratory, Livermore, CA, UCRL-JC-135494 ABS. Prepared for Frontier Science at the NIF: Episode I, Pleasanton, CA, Oct 4, 1999.

MacKinnon, A., Bittner, D., Collins, G., Geddes, C., and Koch, J., Investigation of Layer Uniformity of HD Ice Layers in Cryogenic Spherical Capsules Using a Phase Shifting Interferometer, Lawrence Livermore National Laboratory, Livermore, CA, UCRL-JC-135501 ABS. Prepared for Target Fabrication Mtg '99, Catalina Island, CA, Nov 8, 1999.

Maricle, S., Mouser, R., Schwartz, S., Parham, T., Wegner, P., and Weiland, T., Laser Damage Performance of Fused Silica Optical Components Measured on the Beamlet Laser at $351 \mathrm{~nm}$, Lawrence Livermore National Laboratory, Livermore, CA, UCRL-JC-131221. Prepared for 30th Boulder Damage Symp: Annual Symp on Optical Materials for High Power Lasers, Boulder, CO, Sept 28, 1998.

Marshall, C. D., Erlandson, A., Jancaitis, K., Lawson, J., Sutton, S., and Zapata, L., Performance of National Ignition Facility Prototype Laser Amplifier Facility, Lawrence Livermore National Laboratory, Livermore, CA, UCRL-JC-131986 ABS. Prepared for Lasers and Electro-Optics Society 1998 11th Annual Conf, Orlando, FL, Dec 1, 1998.
McEachern, R., Alford, C., and Jankowski, A., Modified Sputter Deposition Process for ICF Capsule Ablators, Lawrence Livermore National Laboratory, Livermore, CA, UCRL-JC-135588 ABS. Prepared for Target Fabrication Mtg '99, Catalina Island, CA, Nov 8, 1999.

McEachern, R., and Alford, C., Evaluation of Boron-Doped Beryllium as an Ablator for NIF Target Capsules, Lawrence Livermore National Laboratory, Livermore, CA, UCRL-JC-130530; also in Fusion Tech. 35(2), 115-118 (1999).

McEachern, R., Matsuo, J., and Yamada, I., Cluster Ion Beam Polishing for Inertial Confinement Fusion Target Capsules, Lawrence Livermore National Laboratory, Livermore, CA, UCRL-JC-130683.

Prepared for 12th Intl Conf on Ion Implantation Technology, Kyoto, Japan, Jun 22, 1998.

McEachern, R., Tailoring Material Properties of Sputtered Beryllium, Lawrence Livermore National Laboratory, Livermore, CA, UCRL-ID-133589.

Meier, W. R., Systems Modeling and Analysis of Heavy Ion Drivers for Inertial Fusion Energy, Lawrence Livermore National Laboratory, Livermore, CA, UCRL-JC130954; also in Fusion Tech. 34(3), Pt. 2, 326-330 (1998).

Moody, J. D., MacGowan, B. J., Berger, R. L., Estabrook, K. G., Glenzer, S. H., Kirkwood, R. K., Kruer, W. L., Montgomery, D. S., and Stone, G. F., Experimental Study of Laser Beam Transmission and Power Accounting in a Large Scalelength Laser-Plasma, Lawrence Livermore National Laboratory,

Livermore, CA, UCRL-JC-135564.

Submitted to Phys. of Plasmas.

Moody, J. D., MacGowan, B. J., Decker, C. D., Glenzer, S. H., Kirkwood, R. K., Young, P. E., Berger, R. L., Collins, G. W., Geddes, C. G., and Sanchez, J. A., Measurements of SRS and SBS Backscattered Light from $\mathrm{He} / \mathrm{H}_{2}$ Cryogenic Nova Gasbag Targets, Lawrence Livermore National Laboratory, Livermore, CA, UCRL-JC-133942-ABS. Prepared for 29th Annual Anomalous Absorption Conf, Pacific Grove, CA, Jun 13, 1999. 
Moody, J. D., MacGowan, B. J., Glenzer, S. H., Kirkwood, R. K., Kruer, W. L., Pollaine, S. M., Williams, E. A., Stone, G. F., Afeyan, B. B., and Schmitt, A. J., Measurements of Near Forward Scattered Laser Light in a Large Inertial Confinement Fusion Plasma, Lawrence Livermore National Laboratory, Livermore, CA, UCRL-JC-129774; also in Rev. Sci. Inst. 70(1), 677-681 (1999).

Moody, J. D., MacGowan, B. J., Glenzer, S. H., Kirkwood, R. K., Kruer, W. L., Schmitt, A. J., Williams, E. A., and Stone, G. F., First Measurement of Short Length-Scale Density Fluctuations in a Large Laser Plasma, Lawrence Livermore National Laboratory, Livermore, CA, UCRL-JC-133035; also in Phys. Rev. Lett. 83(9), 1783-1786 (1999).

Moody, J. D., Rosmus, W., Schmitt, A. J., Glenzer, S. H., Kirkwood, R. K., MacGowan, B. J., Berger, R. L., Williams, E. A., and Kruer, W. L., Laser Propagation in a High Energy Density Plasma, Lawrence Livermore National Laboratory, Livermore, CA, UCRL-JC-135421 ABS. Prepared for Frontier Science at the NIF: Episode I, Pleasanton, CA, Oct 4, 1999.

Moody, J., MacGowan, B. J., Glenzer, S. H., Kirkwood, R. K., Kruer, W. L., Schmitt, A. J., Williams, E. A., and Stone, G. F., Measurements of Short Scalelength Density Fluctuations in a Large Laser-Plasma, Lawrence Livermore National Laboratory, Livermore, CA, UCRL-JC-135076 ABS. Prepared for 41st Annual Mtg of the American Physical Society Div of Plasma Physics, Seattle, WA, Nov 15, 1999.

Moody, J., Sanchez, J. A., MacGowan, B. J., Young, P. E., Glenzer, S. H., Kirkwood, R. K., Geddes, C. G., Collins, G. A., Decker, C. D., and Suter, L. J., Cryogenic Gasbag Targets for Laser-Plasma Interaction Studies, Lawrence Livermore National Laboratory, Livermore, CA, UCRL-JC-135489 ABS. Prepared for Target Fabrication Mtg '99, Catalina Island, CA, Nov 8, 1999.

Moran, M. J., "The Fusion Diagnostic Gamma Experiment: A High-Bandwidth Fusion Diagnostic of the National Ignition Facility," Rev. Sci. Inst. 70(1) 1226-1228 (1999).
Moran, M., Brown, C. G., Cowan, T. E., Hatchett, S., Hunt, A., Key, M., Pennington, D., Perry, M. D., Phillips, T., and Sangster, T. C., Measurements of $\mathrm{MeV}$ Photon Flashes in Petawatt Laser Experiments, Lawrence Livermore National Laboratory, Livermore, CA, UCRL-JC-131359. Prepared for 40th Annual Mitg of the Div of Plasma Physics, New Orleans, LA, Nov 16, 1998.

Murray, J. E., Milam, D., Boley, C. D., and Estabrook, K. G., Spatial Filter Pinhole Development for the National Ignition Facility, Lawrence Livermore National Laboratory, Livermore, CA, UCRL-JC-134647.

Submitted to Appl. Opt.

\section{$\mathbf{N}$}

Nash, T. J., Derzon, M. S., Chandler, G. A., Leeper, R., Fehl, D., Lash, J., Ruiz, C., Cooper, G., Seaman, J. F., McGurn, J., Lazier, S., Torres, J., Jobe, D., Gilliland, T., Hurst, M., Mock, R., Ryan, P., Nielsen, D., Armijo, J., McKenney, J., Hawn, R., Hebron, D., MacFarlane, J. J., Petersen, D., Bowers, R., Matuska, W., and Ryutov, D. D., "High-Temperature Dynamic Hohlraums on the Pulsed Power Driver Z," Phys. Plasmas 6(5), Pt. 2, 2023-2029 (1999).

Newton, M., and Wilson, M., Main Amplifier Power Conditioning for the National Ignition Facility, Lawrence Livermore National Laboratory, Livermore, CA, UCRL-JC-130975; also in Fusion Tech. 34(3), Pt. 2, 1122-1126 (1998).

Norimatsu, T., Izawa, Y., Mima, K., and Gresho, P. M., "Modeling of the Centering Force in a Compound Emulsion to Make Uniform Plastic Shells for Laser Fusion Targets," Fusion Tech. 35(2), 147-156 (1999).

Norton, M. A., Boley, C. D., Murray, J. E., Sinz, K., and Neeb, K., Long-Lifetime, LowContamination Metal Beam Dumps for NIF Spatial Filters, Lawrence Livermore National Laboratory, Livermore, CA, UCRL-JC-129768. Prepared for 3rd Annual Intl Conf on Solid State Lasers for Application (SSLA) to Inertial Confinement Fusion (ICF), Monterey, CA, Jun 7, 1998. 
Nostrand, M. C., Page, R. H., Payne, S. A., Krupke, W. F., and Schunemann, P. G., Room-Temperature Laser Action at 4.3-4.4 $m m$ in $\mathrm{CaGa}_{2} \mathrm{~S}_{4}: D y^{3+}$, Lawrence Livermore National Laboratory, Livermore, CA, UCRL-JC-133981; also in Optics Lett. 24(17), 1215-1217 (1999).

\section{$\mathrm{O}$}

Olivier, S. S., Gavel, D. T., Friedman, H. W., Max, C. E., An, J. R., Avicola, K., Bauman, B. J., Brase, J. M., Campbell, E. W., and Carrano, C., Improved Performance of the Laser Guide Star Adaptive Optics System at Lick Observatory, Lawrence Livermore National Laboratory, Livermore, CA, UCRL-JC-134956. Prepared for 44th Annual Mtg of the Intl Symp on Optical Science, Engineering, and Instrumentation, Denver, CO, Jul 18, 1999.

Olivier, S. S., Kartz, M. W., Bauman, B. J., Brase, J. M., Brown, C. G., Cooke, J. B., Pennington, D. M., and Silva, D. A., HighResolution Wavefront Control Using Liquid Crystal Spatial Light Modulators, Lawrence Livermore National Laboratory, Livermore, CA, UCRL-JC-134900. Prepared for 44th Annual Mtg of the Intl Symp on Optical Science, Engineering, and Instrumentation, Denver, CO, Jul 18, 1999.

Orthion, P., Roberts, C. C., and Letts, S. A., Optimization of the Evaporator Design for the Fabrication of NIF Polyimide Capsules, Lawrence Livermore National Laboratory, Livermore, CA, UCRL-JC-135594 ABS. Prepared for Target Fabrication $\mathrm{Mtg}$ '99, Catalina Island, CA, Nov 8, 1999.

\section{$\mathbf{P}$}

Papandrew, A. B., Wu, Z. L., and Stolz, C. J., Correlation of Photothermal Signal with Damage Threshold of Hafnia Silica Multilayer High Reflectors at a Single Wavelength and Test Angle, Lawrence Livermore National Laboratory, Livermore, CA, UCRL-JC134940 ABS. Prepared for 31st Boulder Damage Symp: Annual Symp on Optical Materials for High Power Lasers, Boulder, CO, Oct 4, 1999.
Parrish, B., Roberts, C. C., and Letts, S., Smoothing of Polymide NIF Capsules by Gas Levitation, Lawrence Livermore National Laboratory, Livermore, CA, UCRL-JC135544 ABS. Prepared for Target Fabrication Mtg '99, Catalina Island, CA, Nov 8, 1999.

Payne, S. A., and Bibeau, C., Picosecond Nonradiative Processes in Neodymium-Doped Crystals and Glasses: Mechanism for the Energy Gap Law, Lawrence Livermore National Laboratory, Livermore, CA, UCRL-JC-128623; also in J. Luminescence 79(3), 143-159 (1998).

Pennington, D. M., Brown, C. G., Cowan, T. E., Fountain, W., Hatchett, S. P., Henry, E., Johnson, J., Kartz, M., Key, M., and Koch, J., Petawatt Laser Experiments at Laurence Livermore National Laboratory, Lawrence Livermore National Laboratory, Livermore, CA, UCRL-JC-135527.

Submitted to Photonics Science News.

Pennington, D. M., Brown, C. G., Kartz, M., Landon, M., Perry, M. D., and Tietbohl, G., Production of High Intensity Laser Pulses with Adaptive Optics Wavefront Correction, Lawrence Livermore National Laboratory, Livermore, CA, UCRL-JC-132977-ABS-S. Prepared for Intl Conf for Optics XVIII, San Francisco, CA, Aug 2, 1999.

Pennington, D. M., Brown, C. G., Kartz, M., Landon, M., Perry, M. D., and Tietbohl, G., Production of High Intensity Laser Pulses with Adaptive Optics, Lawrence Livermore National Laboratory, Livermore, CA, UCRL-JC-133182-ABS. Prepared for Intl Workshop on Adaptive Optics for Industry and Medicine, Durham, England, Jul 12, 1999.

Pennington, D., Brown, C. G., Cowan, T. E., Fountain, W., Hatchett, S. P., Henry, E., Hunt, A. W., Johnson, J., Kartz, M., Key, M., Koch, J., MacKinnon, A., Moody, J., Parnell, T., Perry, M. D., Phillips, T. W., Roth, M., Sangster, T. C., Snavely, R. A., Stoyer, M., Takahashi, Y., Tsukamoto, M., and Wilks, S. C., Petawatt Experiments at LLNL, Lawrence Livermore National Laboratory, Livermore, CA, UCRL-JC133590-ABS-SUM. Prepared for Applications of High Field and Short Wavelength Sources VIII, Potsdam, Germany, Mar 27, 1999. 
Perry, M. D., Feit, M. D., Barton, I., Dixit, S., Hyde, R., Early, J., and Powell, H. T., Advanced Concepts for the Space Based Laser, Lawrence Livermore National Laboratory, Livermore, CA, UCRL-ID-133277.

Perry, M. D., Sefcik, J. A., Cowan, T., Hatchett, S., Hunt, A., Moran, M., Pennington, D., Snavely, R., and Wilks, S. C., Hard X-Ray Production from High Intensity Laser Solid Interactions, Lawrence Livermore National Laboratory, Livermore, CA, UCRL-JC-130942; also in Rev. Sci. Inst. 70(1), 265-279 (1998).

Perry, M. D., Stuart, B. C., Banks, P. S., Feit, M. D., Yanovsky, V., and Rubenchik, A. M., Ultrashort-Pulse Laser Machining of Dielectric Materials, Lawrence Livermore National Laboratory, Livermore, CA, UCRL-JC-132156; also in J. Appl. Phys. 85(9), 6803-6810 (1999).

Perry, T. S., Klein, R. I., Budil, K. S., and Bach, D. R., Interaction of Laser Driven Shock Waves with a Spherical Density Perturbation, Lawrence Livermore National Laboratory, Livermore, CA, UCRL-JC-132867. Prepared for Joint Working Group (JOWOG37), Los Alamos, NM, Feb 2, 1998.

Petzoldt, R. W., IFE Target Injection and Tracking Experiment, Lawrence Livermore National Laboratory, Livermore, CA, UCRL-LR-120192; also in Fusion Tech. 34(3), Pt. 2, 831-839 (1998).

Phillips, T. W., Cauble, M. D., Cowan, T. E, Hatchett, S. P., Henry, E. A., Key, M. H., Perry, M. D., Sangster, T. C., and Stoyer, M. A., Diagnosing Hot Electron Production by Short Pulse, High Intensity Lasers Using Photonuclear Reactions, Lawrence Livermore National Laboratory, Livermore, CA, UCRL-JC-129813; also in Rev. Sci. Inst. 70(1), 1213-1216 (1999).

Pipes, J. W., Sanchez, J. J., and Burmann, J., Assembly of Cryogenic Hohlraums with Deuterium/Tritium Filled Shells, Lawrence Livermore National Laboratory, Livermore, CA, UCRL-JC-135490 ABS. Prepared for Target Fabrication Mtg '99, Catalina Island, CA, Nov 8, 1999.
Pollaine, S. M., and Nellis, W. J., LaserGenerated Metallic Hydrogen, Lawrence Livermore National Laboratory, Livermore, CA, UCRL-JC-135176. Prepared for Intl Conf on High Pressure Science and Technology, Honolulu, HI, Jul 25-30, 1999.

Pollaine, S., Amendt, P. A., Jones, O., Bradley, D., Landen, O., Wallace, R., Glendinning, G., Turner, R., and Suter, L., $P 6$ and P8 Modes in NIF Hohlraums, Lawrence Livermore National Laboratory, Livermore, CA, UCRL-JC-133888 ABS Rev. Prepared for 41st Annual Mtg of the American Physical Society Div of Plasma Physics, Seattle, WA, Nov 15, 1999.

Porcelli, F., Rossi, E., Cima, G., and Wootton, A., Macroscopic Magnetic Islands and Plasma Energy Transport, Lawrence Livermore National Laboratory, Livermore, CA, UCRL-JC-132568. Prepared for Workshop on Fusion Related Physics and Engineering in Small Devices, Trieste, Italy, Oct 6, 1998.

Powell, H., Inertial Confinement Fusion Quarterly Report, October-December 1998, Volume 9, Number 1, Lawrence Livermore National Laboratory, Livermore, CA, UCRL-LR-105821-99-1.

\section{$\mathbf{R}$}

Regan, S. P., Delettrez, J. A., Bradley, D. K., Glebov, V. Y., Myerhofer, D. D., and Stoeckl, C., Burnthrough Experiments on OMEGA to Study Effects of Laser Irradiation Uniformity and Shine through Layers on Spherical Target Performance, Lawrence Livermore National Laboratory, Livermore, CA, UCRL-JC-135018 ABS. Prepared for 41st Annual Mtg of the American Physical Society Div of Plasma Physics, Seattle, WA, Nov 15, 1999.

Remington, B. A., Arnett, D., Drake, R. P., and Takabe, H., "Modeling Astrophysical Phenomena in the Laboratory with Intense Lasers," Science 284, 1488-1493 (1999). 
Remington, B. A., Budil, K. S., Edwards, M. J., Lasinski, B., Suter, L., and Wan, A. S., Ablation-Front Rayleigh-Taylor Experiments on Al Foils, Lawrence Livermore National Laboratory, Livermore, CA, UCRL-JC135433 ABS. Prepared for Joint Operations Weapons Operations Group 37, Aldermaston, UK, Sep 20, 1999.

Remington, B. A., Extreme States of Matter on Nova, Lawrence Livermore National Laboratory, Livermore, CA, UCRL-ID133627.

Remington, B. A., Hydrodynamics Experiments on Intense Lasers, Lawrence Livermore National Laboratory, Livermore, CA, UCRL-JC-131249 ABS Rev. Prepared for 1999 Centennial Mtg, Atlanta, GA, Mar 20, 1999.

Remington, B. A., Kalantar, D. H., Weber, S. V., Colvin, J. D., Mikaelian, K. O., and Wiley, L., Solid-State Hydrodynamics Experiments on Laser Facilities, Lawrence Livermore National Laboratory, Livermore, CA, UCRL-JC-135440 ABS. Prepared for Joint Operations Weapons Operations Group 37, Aldermaston, UK, Sep 20, 1999.

Remington, B. A., Review of Astrophysics Experiments on Intense Lasers, Lawrence Livermore National Laboratory, Livermore, CA, UCRL-JC-134961 ABS. Prepared for 41st Annual Mtg of the American Physical Society Div of Plasma Physics, Seattle, WA, Nov 15, 1999.

Remington, B. A., Studying the Stars on Earth: Astrophysics on Intense Lasers, Lawrence Livermore National Laboratory, Livermore, CA, UCRL-JC-133464.

Prepared for American Physical Society 1999 Centennial Mtg, Atlanta, GA, Mar 20, 1999.

Rhodes, M. A., Fochs, S., and Biltoft, P., Plasma Electrode Pockels Cell for the National Ignition Facility, Lawrence Livermore National Laboratory, Livermore, CA, UCRL-JC-129710; also in Fusion Tech. 34(3), Pt. 2, 1113-1116 (1998).
Roberts, C. C., Hayward, R., Letts, S. A., and Cook, R. C., Tensile Creep of Polyimide Films, Lawrence Livermore National Laboratory, Livermore, CA, UCRL-JC135495 ABS. Prepared for Target Fabrication Mtg '99, Catalina Island, CA, Nov 8, 1999.

Roberts, C. C., Letts, S. A., Saculla, M., Hsieh, E. J., and Cook, R., Polyimide Films from Vapor Deposition: Toward High Strength, NIF Capsules, Lawrence Livermore National Laboratory, Livermore, CA, UCRL-JC-132194; also in Fusion Tech. 35(2), 138-146 (1999).

Roberts, C. C., Orthion, P., Letts, S. A., Hassel, A., Buckley, S., Fearon, E., and Cook, R. C., Development of Polyimide Ablators for NIF: Analysis of Surface Defects and Generation Mechanism, Lawrence Livermore National Laboratory, Livermore, CA, UCRL-JC-135487 ABS. Prepared for Target Fabrication Mtg '99, Catalina Island, CA, Nov 8, 1999.

Roberts, C. C., Orthion, P., Letts, S. A., Hassel, A., Buckley, S., Fearon, E., Parrish, B., Hayward, R., and Cook, R. C., Development of Polyimide Ablators for NIF: an Overview of Progress at $L L N L$, Lawrence Livermore National Laboratory, Livermore, CA, UCRL-JC-135488 ABS. Prepared for Target Fabrication Mtg '99, Catalina Island, CA, Nov 8, 1999.

Robey, H. F., and Potapenko, S., Ex-Situ Microscopic Observation of the Lateral Instability of Macrosteps on the Surfaces of Rapidly Grown $\mathrm{KH}_{2} \mathrm{PO}_{4}$ Crystals, Lawrence Livermore National Laboratory, Livermore, CA, UCRL-JC-133360. Submitted to J. Cryst. Growth.

Robey, H. F., Floyd, R., Torres, R., and Burnham, A., Impurity Leaching Rates of 1000 Liter Growth Tanks, Lawrence Livermore National Laboratory, Livermore, CA, UCRL-ID-133365.

Robey, H. F., Hawley-Fedder, R., Floyd, R., DeHaven, M., and Rozsa, R., LLNL KDP Crystal Growth Electronic Log Book, Lawrence Livermore National Laboratory, Livermore, CA, UCRL-MA-133359. 
Robey, H. F., Potapenko, S. U., and Summerhays, K. D., "Bending" of Steps on Rapidly Grown $\mathrm{KH}_{2} \mathrm{PO}_{4}$ Crystals due to an Inhomogeneous Surface Supersaturation Field, Lawrence Livermore National Laboratory, Livermore, CA, UCRL-JC-133206.

Submitted to J. Cryst. Growth.

Robey, H., Burke, G., Edwards, J., Glendinning, S. G., Greenough, J., Hsing, W., Klem, D., Miller, P., Remington, B., and Stry, P., Hydrodynamic Scaling Issues, Lawrence Livermore National Laboratory, Livermore, CA, UCRL-JC-134968 ABS. Prepared for 1999 Nuclear Explosives Design Physics Conf, Los Alamos, NM, Oct 25, 1999.

Robey, H., Remington, B. A., Kane, J. O., Hurrican, O., Drake, R. P., Teyssier, R., and Knauer, J., Recent Results from Astrophysically Relevant Hydrodynamic Experiments on the OMEGA Laser, Lawrence Livermore National Laboratory, Livermore, CA, UCRL-JC-134969 ABS. Prepared for 41st Annual Mtg of the American Physical Society Div of Plasma Physics, Seattle, WA, Nov 15, 1999.

Rosen, M. D., The Physics Issues That Determine Inertial Confinement Fusion Target Gain and Driver Requirements: A Tutorial, Lawrence Livermore National Laboratory, Livermore, CA, UCRL-JC-131381; also in Phys. Plasmas 6(5), Pt. 2, 1690-1699 (1999).

Rossetter, E., Electronics Cooling in a Diagnostic Instrument Manipulator, Lawrence Livermore National Laboratory, Livermore, CA, UCRL-ID-132225.

Runkel, M., and Sharp, R., Modeling KDP Bulk Damage Curves for Prediction of LargeArea Damage Performance, Lawrence Livermore National Laboratory, Livermore, CA, UCRL-JC-134766 ABS. Prepared for 31st Boulder Damage Symp: Annual Symp on Optical Materials for High Power Lasers, Boulder, CO, Oct 4, 1999.

Ryutov, D. D., Drake, R. P., and Remington, B. A., Criteria for Scaled Laboratory Simulations of Astrophysical MHD Phenomena, Lawrence Livermore National Laboratory, Livermore, CA, UCRL-JC-133092. Submitted to Astrophys. J. Supplements.
Ryutov, D., Drake, R. P., Kane, J., Liang, E., Remington, B. A., and Wood-Vasey, W. M., Similarity Criteria for the Laboratory Simulations of Supernova Hydrodynamics, Lawrence Livermore National Laboratory, Livermore, CA, UCRL-JC-130956; also in Astrophys. J. 518(N2PT1), 821-832 (1999).

\section{$\mathbf{S}$}

Sanchez, J. J., and Giedt, W. H., Thermal Control of Cryogenic Cylindrical Hohlraums for Indirect-Drive Inertial Confinement Fusion, Lawrence Livermore National Laboratory, Livermore, CA, UCRL-JC132168. Submitted to J. Appl. Phys.

Sanchez, J., Giedt, W., Pipes, J., and Moody, J. D., Multi-Path Equalizer for Maintaining Uniform Azimuthal Heat Flow in a Cylindrical NIF Hohlraum, Lawrence Livermore National Laboratory, Livermore, CA, UCRL-JC-135591 ABS. Prepared for Target Fabrication Mtg '99, Catalina Island, CA, Nov 8, 1999.

Sanchez, J., Operational Analysis and Review of Thermally Shimmed Cryogenic Hohlraums Design, Lawrence Livermore National Laboratory, Livermore, CA, UCRL-JC135497 ABS. Prepared for Target Fabrication Mtg '99, Catalina Island, CA, Nov 8, 1999.

Sankaran, V., Everett, M. J., Maitland, D. J., and Walsh, J. T., Comparison of PolarizedLight Propagation in Biological Tissue and Phantoms, Lawrence Livermore National Laboratory, Livermore, CA, UCRL-JC133624; also in Optics Lett. 24(15), 1044-1046 (1999).

Sankaran, V., Schonenberger, K., Walsh, Jr., J. T., and Maitland, D. J., Polarization Discrimination of Coherently Propagating Light in Turbid Media, Lawrence Livermore National Laboratory, Livermore, CA, UCRL-JC-132681; also in Appl. Optics 38(19), 4252-4261 (1999).

Sater, J., Kozioziemski, B., Collins, G. W., Mapoles, E. R., Pipes, J., Burmann, J., and Bernat, T. P., Cryogenic D-T Fuel Layers Formed in $1 \mathrm{~mm}$ Spheres by Beta-Layering, Lawrence Livermore National Laboratory, Livermore, CA, UCRL-JC-128031. Prepared for 40th Annual Mtg of the Div of Plasma Physics, New Orleans, LA, Nov 16, 1998. 
Sater, J., Kozioziemski, B., Everett, M., Burmann, J., and Pipes, J., Formation of Smooth DT Layers at Temperatures Well Below the Triple Point, Lawrence Livermore National Laboratory, Livermore, CA, UCRL-JC-135485 ABS. Prepared for Target Fabrication Mtg '99, Catalina Island, CA, Nov 8, 1999.

Sathyam, U. S., Colston Jr., B. W., Da Silva, L. B., and Everett, M. J., Evaluation of Optical Coherence Quantitation of Analytes in Turbid Media by Use of Two Wavelengths, Lawrence Livermore National Laboratory, Livermore, CA, UCRL-JC-131433; also in Appl. Optics 38(10), 2097-2104 (1999).

Sawicki, R., Bowers, J., Hackel, R., Larson, D., Manes, K., and Murray, J., Engineering the National Ignition Facility, Lawrence Livermore National Laboratory, Livermore, CA, UCRL-JC-131522; also in Fusion Tech. 34(3), Pt. 2, 1097-1104 (1998).

Schwartz, S., Feit, M. D., Kozlowski, M. R., and Mouser, R. P., Current 3w Large Optic Test Procedures and Data Analysis for the Quality Assurance of National Ignition Facility, Lawrence Livermore National Laboratory, Livermore, CA, UCRL-JC131217. Prepared for 30th Boulder Damage Symp: Annual Symp on Optical Materials for High Power Lasers, Boulder, CO, Sept 28, 1998.

Sheehan, L., NIF Small Optics Laser Damage Test Specifications, Lawrence Livermore National Laboratory, Livermore, CA, UCRL-ID-133979 Rev 1.

Sheehan, L., Schwartz, S., Battersby, C., Dickson, R., Jennings, R., Kimmons, J., Kozlowski, M., Maricle, S., Mouser, R., and Runkel, M., Automated Damage Test Facilities for Materials Development and Production Optic Quality Assurance at Lawrence Livermore National Laboratory, Lawrence Livermore National Laboratory, Livermore, CA, UCRL-JC-131230. Prepared for 30th Boulder Damage Symp: Annual Symp on Optical Materials for High Power Lasers, Boulder, CO, Sept 28, 1998.
Shestakov, A. I., Time Dependent Simulations of Point Explosions with Heat Conduction, Lawrence Livermore National Laboratory, Livermore, CA, UCRL-JC-132414; also in Phys. Plasmas 11(5), 1091-1095 (1999).

Shigemori, K., Ditmire, T., Edwards, M. J., Estabrook, K., Remington, B. A., Rubenchik, A. M., and Ryutov, D. D., Creating Radiative Blast Wave with Intense Lasers, Lawrence Livermore National Laboratory, Livermore, CA, UCRL-JC135614 ABS. Prepared for Frontier Science at the NIF: Episode I,. Pleasanton, CA, Oct 4, 1999.

Shigemori, K., Ditmire, T., Remington, B. A., Estabrook, K. G., Farley, D. R., Ryutov, D. D., Rubenchik, A. M., Kodama, R., Koase, T., and Ochi, Y., Radiative Hydrodynamics Experiments for Astrophysics, Lawrence Livermore National Laboratory, Livermore, CA, UCRL-JC-133835-ABS. Prepared for 29th Annual Anomalous Absorption Conf, Pacific Grove, CA, Jun 13, 1999.

Shigemori, K., Ditmire, T., Remington, B. A., Yanovsky, V., Ryutov, D., Estabrook, K. G., Edwards, M. J., Rubenchik, A. M., Liang, E., and Keilty, K. A., Observation of the Transition from Hydrodynamic to Radiative Shocks, Lawrence Livermore National Laboratory, Livermore, CA, UCRL-JC-134470 Rev 1. Submitted to Astrophys. J.

Shigemori, K., Estabrook, K. G., Farley, D. R., Kodama, R., Koase, T., Ochi, Y., Remington, B. A., Stone, J., Turner, N., Developing Astrophysical Jet Experiments on Lasers, Lawrence Livermore National Laboratory, Livermore, CA, UCRL-JC135572 ABS. Prepared for Frontier Science at the NIF: Episode I, Pleasanton, CA, Oct 4, 1999.

Shigemori, K., Farley, D. R., Remington, B. A., Estabrook, K. G., Kodama, R., Koase, T., Ochi, Y., and Azechi, H., Radiatively Cooled Jet Experiments Using High Intensity Lasers, Lawrence Livermore National Laboratory, Livermore, CA, UCRL-JC134962 ABS. Prepared for 41st Annual Mtg of the American Physical Society Div of Plasma Physics, Seattle, WA, Nov 15, 1999. 
Small IV, W., Celliers, P. M., Da Silva, L. B., Matthews, D. L., and Soltz, B. A., Two-Color Mid-Infrared Thermometer Using a Hollow Glass Optical Fiber, Lawrence Livermore National Laboratory, Livermore, CA, UCRL-JC-128082 Rev 1; also in Appl. Opt. 37(28), 6677-6683 (1998).

Smolsky, I. L., Zaitseva, N. P., Carman, L., Ryon, R. W., and Voloshin, A. E., Mechanisms of Vicinal Sectorality Formation on Dipyramid Faces in the KDP Group Crystals, Lawrence Livermore National Laboratory, Livermore, CA, UCRL-JC133460. Submitted to J. Cryst. Growth.

Sommer, S., Trummer, D. J., MacCalden, P. B., and Gerhard, M. A., Seismic Analyses of NIF Structures, Lawrence Livermore National Laboratory, Livermore, CA, UCRL-JC-134210-ABS. Prepared for 3rd Biennial Tri-Laboratory Engineering Conf on Modeling \& Simulation, Pleasanton, CA, Nov 3, 1999.

Stephens, R. B., Key, M. H., Koch, J., and Pennington, D., X-Ray Imaging to Characterize MeV Electrons Propagation through Plastic Targets, Lawrence Livermore National Laboratory, Livermore, CA, UCRL-JC-135478. Prepared for 1st Intl Conf on Inertial Fusion Sciences and Applications, Bordeaux, France, Sep 12, 1999.

Still, C. H., Langer, S. H., Alley, W. E., and Zimmerman, G. B., Scientific ProgrammingShared Memory Programming with Open MP, Lawrence Livermore National Laboratory, Livermore, CA, UCRL-JC-131544; also in Comput. Phys. 12(6), 577-584 (1998).

Stolz, C. J., Sheehan, L. M., Maricle, S. M., Schwartz, S., and Hue, J., Study of Laser Conditioning Methods of Hafnia Silica Multilayer Mirrors, Lawrence Livermore National Laboratory, Livermore, CA, UCRL-JC-131215. Prepared for 30th Boulder Damage Symp: Annual Symp on Optical Materials for High Power Lasers, Boulder, CO, Sept 28, 1998.
Stone, J. M., Turner, N., Estabrook, K., Remington, B., Farley, D., and Glendinning, S. G., Testing Astrophysical Radiation Hydrodynamics Codes with Hypervelocity Jet Experiments on the Nova Laser, Lawrence Livermore National Laboratory, Livermore, CA, UCRL-JC135789. Prepared for 2 nd Intl Workshop on Laboratory Astrophysics with Intense Lasers, Tucson, AZ, March 19, 1998.

Strobel, G. L., Haan, S. W., Dittrich, T. R., Hinkel, D. E., and Marinak, M. M., Sensitivity of NIF Ignition Target Performance to Central DT Gas Density, Lawrence Livermore National Laboratory, Livermore, CA, UCRL-JC-134933 ABS. Prepared for 41st Annual Mtg of the American Physical Society Div of Plasma Physics, Seattle, WA, Nov 15, 1999.

Suter, L. J., Landen, O. L., and Koch, J. I., Prospects for Fluorescence Based Imaging/Visualization of Hydrodynamic Systems on the National Ignition Facility, Lawrence Livermore National Laboratory, Livermore, CA, UCRL-JC-130980; also in Rev. Sci. Inst. 70(1) 663-666 (1999).

Suter, L. J., Progress in NIF Ignition Capsule Coupling Efficiency, Lawrence Livermore National Laboratory, Livermore, CA, UCRL-JC-135021 ABS. Prepared for 41st Annual Mtg of the American Physical Society Div of Plasma Physics, Seattle, WA, Nov 15, 1999.

Suter, L., Glenzer, S., Turner, R., Decker, C., Landen, O., Lasinski, B., MacGowan, B., Dattolo, E., Dague, N., and Juraszek, D., Status of Our Understanding and Modeling of NIF X-Ray Coupling Efficiency, Lawrence Livermore National Laboratory,

Livermore, CA, UCRL-ID-132922.

Suter, L., Porter, J., Bourgaude, J., Kauffman, R., Simmons, W., and Turner, R., Requirements for NIF's Soft X-Ray Drive Diagnostic, Lawrence Livermore National Laboratory, Livermore, CA, UCRL-JC133889-ABS. Prepared for 29th Annual Anomalous Absorption Conf, Pacific Grove, CA, Jun 13, 1999. 
Suter, L., Van Wonterghem, B., Rothenberg, J., Munro, D., Haan, S., Pollaine, S., and Tabak, M., Feasibility of High Yield/ High Gain NIF Capsules, Lawrence Livermore National Laboratory, Livermore, CA, UCRL-JC-133887 ABS Rev. Prepared for Nuclear Explosives Design Physics Conf, Los Alamos, NM, Oct 25, 1999.

\section{$\mathrm{T}$}

Tabak, M., Inertial Fusion Energy (IFE) Concepts Target Physics Subgroup, Lawrence Livermore National Laboratory, Livermore, CA, UCRL-JC-135518. Prepared for Fusion Summer Study, Snowmass, CO, Jul 11, 1999.

Takagi, M., Cook, R., Stephens, R., and Paguio, S., Stiffening of P $\alpha M S$ Mandrels during Curing, Lawrence Livermore National Laboratory, Livermore, CA, UCRL-JC-135545 ABS. Prepared for Target Fabrication Mtg '99, Catalina Island, CA, Nov 8, 1999.

Takagi, M., Cook, R., Stephens, R., Gibson, J., and Paguio, S., Decreasing Out-of-Round in P $\alpha M S$ Mandrels by Increasing Interfacial Tension, Lawrence Livermore National Laboratory, Livermore, CA, UCRL-JC135539 ABS. Prepared for Target Fabrication Mtg '99, Catalina Island, CA, Nov 8, 1999.

Takagi, M., Cook, R., Stephens, R., Gibson, J., and Paguio, S., Effects of Controlling Osmotic Pressure on a PoMS Microencapsulated Shell During Curing, Lawrence Livermore National Laboratory, Livermore, CA, UCRL-JC-135540 ABS. Prepared for Target Fabrication Mtg '99, Catalina Island, CA, Nov 8, 1999.

Tartt, K. A., Kruer, W. L., and Wilks, S. C., Ultra-Intense Laser Plasma Effects: Gigagauss Magnetic Fields, Relativistic Electron Beams, and Ultra-High Target Potentials, Lawrence Livermore National Laboratory, Livermore, CA, UCRL-JC-133789 ABS Rev. Prepared for 41st Annual Mtg of the American Physical Society Div of Plasma Physics, Seattle, WA, Nov 15, 1999.
Tatartchenko, V., and Beriot, E., Growth of Large KDP Crystals in the Form of Plates, Lawrence Livermore National Laboratory, Livermore, CA, UCRL-JC-132196.

Prepared for 3rd Annual Intl Conf on Solid State Lasers for Application (SSLA) to Inertial Confinement Fusion (ICF), Monterey, CA, Jun 7, 1998.

Tearney, W., National Ignition Facility Operations Training Plan, Lawrence Livermore National Laboratory, Livermore, CA, UCRL-MA-132903-DR-RE.

Tobin, M. T., and Eder, D. C., Modeling Debris Shield Replacement for ICF Facilities, Lawrence Livermore National Laboratory, Livermore, CA, UCRL-JC-133646-ABS. Prepared for 1st Intl Conf on Inertial Fusion Sciences and Applications, Bordeaux, France, Sept 12, 1999.

Tobin, M., Eder, D., Thomas, I., Latkowski, J., Brereton, B., MacGowan, B., Horton, R., Duewer, T., Zaka, F., and Davis, S., Debris Shield Survivability and Lifetimes for NIF, Lawrence Livermore National Laboratory, Livermore, CA, UCRL-JC-135650.

Prepared for 1st Intl Conf on Inertial Fusion Sciences and Applications, Bordeaux, France, Sep 13, 1999.

Turner, R. E., Amendt, P. A., Bradley, D., Landen, O., Geddes, C., Haan, S., Suter, L. J., Wallace, R., Morse, S., and Pien, G., High-Convergence Indirect-Drive Implosions on OMEGA: Experiment, Lawrence Livermore National Laboratory, Livermore, CA, UCRL-JC-135019 ABS. Prepared for 41st Annual Mtg of the American Physical Society Div of Plasma Physics, Seattle, WA, Nov 15, 1999.

Turner, R. E., Amendt, P., Landen, O. L., Glendinning, S. G., Bell, P., Decker, C., Hammel, B. A., Kalantar, D., Lee, D., and Wallace, R., Demonstration of Time Dependent Symmetry Control in Hohlraums by the Use of Drive Beam Staggering, Lawrence Livermore National Laboratory, Livermore, CA, UCRL-JC-132330.

Submitted to Phys. Rev. Lett. 
Turner, R. E., Landen, O. L., Bell, P., Costa, R., and Hargrove, D., Achromatically Filtered Diamond Photoconductive Detectors for High Power Soft X-Ray Flux. Measurements, Lawrence Livermore National Laboratory, Livermore, CA, UCRL-JC-129713; also in Rev. Sci. Inst. 70(1), 656-658 (1999).

\section{$\mathbf{U}$}

Unites, W. G., Pipes, J., Burmann, J., Jones, R., Da Silva, L., Celliers, P., and Collins, G. W., Cryogenic Cell Target for Equation of State Measurements in Deuterium, Lawrence Livermore National Laboratory,

Livermore, CA, UCRL-JC-129952 ABS Rev. Prepared for Target Fabrication Mtg '99, Catalina Island, CA, Nov 8, 1999.

\section{V}

Van Wonterghem, B., Hibbard, W., Kalantar, D., Lee, F. D., MacGowan, B. J., Pittenger, L., Tobin, M., and Wong, K., Target Experimental Area and Systems of the National Ignition Facility (NIF), Lawrence Livermore National Laboratory, Livermore, CA, UCRL-JC-134007-ABS. Prepared for 1st Intl Conf on Inertial Fusion Sciences and Applications, Bordeaux, France, Sept 12, 1999.

\section{W}

Wallace, J. M., Murphy, T. J., Delamater, N. D., Klare, K. A., Oertel, J. A., Magelssen, G. R., Lindman, E. L., Hauer, A. A., Gobby, P., Schnittman, J. D., Craxton, R. S., Seka, W., Kremens, R., Bradley, D., Pollaine, S. M., Turner, R. E., Landen, O. L., Drake, D., and MacFarlane, J. J., "Inertial Confinement Fusion with Tetrahedral Hohlraums at OMEGA," Phys. Rev. Lett. 82(19), 3807-3810 (1999).

Wallace, R., Ross, T., and Rivers, C., Low Cost DOT Certified Pressurized ICF Target Transport System, Lawrence Livermore National Laboratory, Livermore, CA, UCRL-JC-135541 ABS. Prepared for Target Fabrication Mtg '99, Catalina Island, CA, Nov 8, 1999.
Weakley, S. C., Stolz, C. J., Wu, Z. L., Bevis, R. P., and von Gunten, M. K., Role of Starting Material Composition in Interfacial Damage Morphology of Hafnia Silica Multilayer Coatings, Lawrence Livermore National Laboratory, Livermore, CA, UCRL-JC-131214. Prepared for 30th Boulder Damage Symp: Annual Symp on Optical Materials for High Power Lasers, Boulder, CO, Sept 28, 1998.

Weber, S. V., Kalantar, D. H., Colvin, J. D., Gold, D. M., Mikaelian, K. O., Remington, B. A., and Wiley, L. G., Nova Experiments. Examining Rayleigh-Taylor Instability in Materials with Strength, Lawrence Livermore National Laboratory, Livermore, CA, UCRL-JC-132739 ABS. Prepared for 7th Intl Workshop on the Physics of Compressible Turbulent Mixing, St. Petersburg, Russia, Jul 5, 1999.

Weber, S. V., Kalantar, D. H., Colvin, J. D., Gold, D. M., Mikaelian, K. O., Remington, B. A., Wiley, L. G., Loveridge, A., Wark, J. S., and Meyers, M. A., Nova Experiments on Rayleigh-Taylor Instabilities in Solids, Lawrence Livermore National Laboratory, Livermore, CA, UCRL-JC-134935 ABS.

Prepared for 41st Annual Mtg of the American Physical Society Div of Plasma Physics, Seattle, WA, Nov 15, 1999.

Wegner, P. J., Auerbach, J. M., Barker, C. E., Burkhart, S. C., Couture, S. A., De Yoreo, J. J., Hibbard, R. L., Liou, L. W., Norton, M. A., and Whitman, P. A., Frequency Converter Development for the National Ignition Facility, Lawrence Livermore National Laboratory, Livermore, CA, UCRL-JC-129725. Prepared for 3rd Intl Conf on Solid State Lasers for Application (SSLA) to Inertial Confinement Fusion (ICF), Monterey, CA, Jun 7, 1998.

Wegner, P. J., Henesian, M. A., Salmon, J. T., Seppala, L. G., Weiland, T. L., Williams, W. H., and Van Wonterghem, B. M., Wavefront and Divergence of the Beamlet Prototype Laser, Lawrence Livermore National Laboratory, Livermore, CA, UCRL-JC-129724. Prepared for 3rd Intl Conf on Solid State Lasers for Application (SSLA) to Inertial Confinement Fusion (ICF), Monterey, CA, Jun 7, 1998. 
Wharton, K. B., Kirkwood, R. K., Glenzer, S. H., Estabrook, K. G., Afeyan, B. B., Cohen, B. I., Moody, J. D., MacGowan, B. J., and Joshi, C., Observation of Resonant Energy Transfer between Identical-Frequency Laser Beams, Lawrence Livermore National Laboratory, Livermore, CA, UCRL-JC130320; also in Phys. Plasmas 6(5), Pt. 2, 2144-2149 (1999).

Wharton, K. B., Laser-Plasma Interactions Relevant to Inertial Confinement Fusion, Lawrence Livermore National Laboratory, Livermore, CA, UCRL-LR-132566.

Wheeler, E. K., McWhirter, J., Whitman, P. K., De Yoreo, J., and Hester, M., Scatter Loss from Environmental Degradation of KDP Crystals, Lawrence Livermore National Laboratory, Livermore, CA, UCRL-JC134819 ABS. Prepared for 31st Boulder Damage Symp: Annual Symp on Optical Materials for High Power Lasers, Boulder, CO, Oct 4, 1999.

Whitman, P. K., Bletzer, K., Hendrix, J. L., Genin, F. Y., Hester, M., and Yashiyama, J., Laser-Induced Damage of Absorbing and Diffusing Glass Surfaces under IR and UV Irradiation, Lawrence Livermore National Laboratory, Livermore, CA, UCRL-JC131213. Prepared for 30th Boulder Damage Symp: Annual Symp on Optical Materials for High Power Lasers, Boulder, CO, Sept 28, 1998.

Wilhelmsen, J., and Thompson, C. A., Adaptive Optic for Correcting Low-Order Wavefront Aberrations, Lawrence Livermore National Laboratory, Livermore, CA, UCRL-ID-135689.

Wilks, S. C., Liang, E. P., and Tabak, M., Pair Production by Ultraintense Lasers, Lawrence Livermore National Laboratory, Livermore, CA, UCRL-JC-124499 Rev 1. Submitted to Phys. Rev. Lett.

Williams, E. A., Bending and Spreading of RPP/SSD Laser Beams, Lawrence Livermore National Laboratory, Livermore, CA, UCRL-JC-132989-ABS. Prepared for 3rd Intl Workshop on Laser Plasma Interaction Physics, Banff, Canada, Feb 17, 1999.
Williams, E. A., Berger, R. L., Langdon, A. B., and Still, C. H., Scaling of Stimulated Raman and Brillouin Backscattering with Speckle Size, Lawrence Livermore National Laboratory, Livermore, CA, UCRL-JC135009 ABS. Prepared for 41st Annual Mtg of the American Physical Society Div of Plasma Physics, Seattle, WA, Nov 15, 1999.

Williams, E. A., Langdon, A. B., Still, C. H., Berger, R. L., and Dixit, S. N., F3D Simulations of the Propagation of Nova Beams in Gasbag Targets, Lawrence Livermore National Laboratory, Livermore, CA, UCRL-JC-133775-ABS. Prepared for 29th Annual Anomalous Absorption Conf, Pacific Grove, CA, Jun 13, 1999.

Williams, W., FSFIN, Version 1.0, Lawrence Livermore National Laboratory, Livermore, CA, UCRL-CODE-99022.

Williams, W., GMPFIN, Version 1.0, Lawrence Livermore National Laboratory, Livermore, CA, UCRL-CODE-99021.

Williams, W., Lawson, J., and Henesian, M., PHOMGLASS-Interferometry Analysis Software for Laser Glass Slab Blanks, Lawrence Livermore National Laboratory, Livermore, CA, UCRL-CODE-99001.

Williams, W., MPCOAT, version 1.0, Lawrence Livermore National Laboratory, Livermore, CA, UCRL-CODE-99032.

Wootton, A. J., Fusion Research and the Developing Nations, Lawrence Livermore National Laboratory, Livermore, CA, UCRL-JC-134097. Submitted to Phys. Community News.

Wootton, A., National Facility Physics and Diagnostics, Lawrence Livermore National Laboratory, Livermore, CA, UCRL-JC135263. Prepared for Laser Aided Plasma Diagnostics, Lake Tahoe, CA, Sep 26, 1999.

Wu, Z. L., Feit, M. D., Kozlowski, M. R., Rubenchik, A. M., and Sheehan, L., Laser Modulated Scattering as a Nondestructive Evaluation Tool for Optical Surfaces and Thin Film Coatings, Lawrence Livermore National Laboratory, Livermore, CA, UCRL-JC-131211. Prepared for 30th Boulder Damage Symp: Annual Symp on Optical Materials for High Power Lasers, Boulder, CO, Sept 28, 1998. 
Wu, Z. L., Feit, M. D., Kozlowski, M., Natoli, J. Y., Rubenchik, A. M., Sheehan, L., and Yan, M., Single-Beam Photothermal Microscopy-a New Diagnostic Tool for Optical Materials, Lawrence Livermore National Laboratory, Livermore, CA, UCRL-IC-131212. Prepared for 30th Boulder Damage Symp: Annual Symp on Optical Materials for High Power Lasers, Boulder, CO, Sept 28, 1998.

\section{$\mathbf{Y}$}

Young, P. E., Berger, R. L., Decker, C., Divol, L., Kirkwood, R. K., Geddes, C., Glenzer, S. H., Hinkel, D. E., Langdon, A. B., MacGowan, B. J., Moody, J. D., Rothenberg, J. E., Still, C. H., Suter, L., and Williams, E. A., Recent Laser-Plasma Interaction Studies at LLNL, Lawrence Livermore National Laboratory, Livermore, CA, UCRL-JC-133633. Prepared for 1st Intl Conf on Inertial Fusion Sciences and Applications, Bordeaux, France, Sep 12-19, 1999.

Young, P. E., Moody, J. D., Rozmus, W., Russell, D. A., and DuBois, D. F., Angular Dependence of $3 \omega_{0} / 2$ Spectra near the TwoPlasmon Decay Threshold, Lawrence Livermore National Laboratory, Livermore, CA, UCRL-JC-133630-ABS. Prepared for 1st Intl Conf on Inertial Fusion Sciences and Application, Bordeaux, France, Sept 12, 1999.

\section{$\mathbf{Z}$}

Zaitseva, N., Atherton, J., Rozsa, R., Carman, L., Smolsky, I., Runkel, M., Ryon, R., and James, L., "Design and Benefits of Continuous Filtration in Rapid Growth of Large KDP and DKDP Crystals," J. Crystal Growth 197(4), 911-920 (1999).

Zaitseva, N., Carman, L., Smolsky, I., Torres, R., and Yan, M., Effect of Impurities and Supersaturation on the Rapid Growth of KDP Crystals, Lawrence Livermore National Laboratory, Livermore, CA, UCRL-JC-132677 Rev 1. Submitted to J. of Crystal Growth.

Zaitseva, N., Smolsky, I., Carman, L., and Ryon, R., Rapid Crystal Growth from Solutions: Mechanisms of Growth and Defect Formation, Lawrence Livermore National Laboratory, Livermore, CA, UCRL-JC133048-ABS. Prepared for 18th Intl Congress of Crystallography, Glasgow, Scotland, Aug 4, 1999.

Ze, F., Landen, O. L., Bell, P. M., Turner, R. E., Tutt, T., Alvarez, S. S., and Costa, R. L., Investigation of Quantum Efficiencies in Multilayered Photocathodes for Microchannel Plate Applications, Lawrence Livermore National Laboratory, Livermore, CA, UCRL-JC-129620; also in Rev. Sci. Inst. 70(1), 659-662 (1999).

Zweiback, J., Ditmire, T., and Perry, M. D., Femtosecond Time-Resolved Studies of the Dynamics of Noble-Gas Cluster Explosions, Lawrence Livermore National Laboratory, Livermore, CA, UCRL-JC-131241; also in Phys. Rev. A 59(5), R3166-R3169 (1999). 